\title{
Behavior Genetics Association 51st Annual Meeting Abstracts
}

\section{The Genetic and Phenotypic Architecture of Playing Video Games}

Abdel Abdellaoui ${ }^{1}$, Joelle Pasman ${ }^{2}$, Jorien L. Treur ${ }^{1}$, Laura W. Wesseldijk ${ }^{1}$, Shu Liu ${ }^{1}$, Perline A. Demange ${ }^{3}$, Dirk J.A. Smit ${ }^{1}$, Michel G. Nivard ${ }^{3}$, Karin J.H. Verweij ${ }^{1}$ ${ }^{1}$ Department of Psychiatry, Amsterdam UMC,

University of Amsterdam, Amsterdam, The Netherlands

${ }^{2}$ Behavioural Science Institute, Radboud University Nijmegen, The Netherlands

${ }^{3}$ Department of Biological Psychology, VU University, Amsterdam, The Netherlands

Keywords GWAS, Mendelian Randomization, Video Games Playing games has always been a universal component of human behavior. Games are usually played for entertainment, but serve as an important tool for social bonding and education as well. Since the advent of video games, they have become an increasingly popular leisure activity. While playing games is generally considered healthy human behavior, the reputation of playing video games is mixed. Many studies show positive relationships between playing video games and cognitive processes, but there is also support for adverse effects on behavior and mental health by excessive use of video games. Few studies have focused on explaining individual differences in playing video games. In this study, we use genome-wide data and a large number of phenotypic measurements in $\sim 450 \mathrm{k}$ adults to investigate the associations between genes, behavior, mental health, and playing video games. We perform genome-wide and phenomewide association studies and investigate causal relationships using Mendelian Randomization and sibling fixed effects designs. With these analyses, we aim to better understand (a) why some people play video games more often than others; and (b) if there are causal relationships between playing video games, cognition, and mental health and, if so, in what direction.

Grant Support: Foundation Volksbond Rotterdam.

\section{Household Chaos and Childhood ADHD Symptoms: A Gene-Environment Correlation Study}

Jessica Agnew-Blais, ${ }^{1}$ Daniel W. Belsky, ${ }^{2}$ Andrea Danese, ${ }^{1,3}$ Guilherme V. Polanczyk, ${ }^{4}$ Karen Sugden, ${ }^{5,6}$ Jasmin Wertz, ${ }^{5}$ Benjamin Williams ${ }^{5,6}$ Cathryn M. Lewis, ${ }^{1}$ Louise Arseneault, ${ }^{1}$ Terrie E. Moffitt ${ }^{1,5,7}$

${ }^{1}$ Social, Genetic, and Developmental Psychiatry Centre, Institute of Psychiatry, Psychology, and Neuroscience, King's College London, London UK

${ }^{2}$ Department of Epidemiology, Columbia Mailman School of Public Health, New York, NY, USA

${ }^{3}$ South London and Maudsley NHS Foundation Trust, London, UK

${ }^{4}$ University of São Paulo Medical School, São Paulo, Brazil

${ }^{5}$ Department of Psychology and Neuroscience, Duke University, Durham, NC, USA

${ }^{6}$ Center for Genomic and Computational Biology, Duke University, Durham, NC, USA

${ }^{7}$ Department of Psychiatry and Behavioral Sciences, Duke University, Durham, NC, USA

Keywords ADHD, Longitudinal, Gene-environment correlation, Polygenic risk score

Environmental risk factors associated with ADHD include prenatal maternal smoking and aspects of the home environment (e.g. chaotic household). However, such risk factors may be more likely to occur when parents have higher genetic risk for ADHD. This study investigates gene-environment correlation (rGE) by assessing the association between maternal ADHD genetic risk and household chaos, and by examining joint effects of household chaos and ADHD genetic risk on level and longitudinal change in ADHD symptoms across childhood. Participants were from the Environmental Risk (ERisk) Longitudinal Twin Study, a population-based birth cohort of 2232 twins. ADHD was assessed at ages 5, 7, 10, and 12. Household chaos was rated by research workers at ages 7, 10 and 12, and by moms and children at age 12 . Sensitivity analysis to estimate the extent of rGE was assessed using GSens. Research-worker-reported household chaos was significantly predicted by maternal ADHD PRS. Maternal ADHD PRS was also significantly associated with baseline levels of offspring ADHD symptoms, but not with change in symptoms over time. Household chaos was significantly associated with baseline ADHD symptoms and with a slower rate of improvement in 
symptoms from childhood to adolescence. We found evidence of passive and active rGE via associations between mother's and offspring's ADHD PRS and household chaos. Children with high levels of both ADHD genetic risk and household chaos had the most elevated course of ADHD symptoms from early childhood to adolescence compared to those with either high genetic or environment risk alone.

Grant Support: JAB is an MRC Skills Development Fellow.

\section{Increased Burden of Common Risk Variants Does Not Account for the High Recurrence Risk of Schizophrenia in Multiplex Families}

Mohammad Ahangari ${ }^{1,2}$, Amanda E. Gentry ${ }^{1}$, Robert Kirkpatrick ${ }^{1,3}$, Brian C. Verrelli ${ }^{4}$, Tan-Hoang Nguyen ${ }^{1,3}$, Kenneth S. Kendler ${ }^{1,3,5}$, Silviu-Alin Bacanu ${ }^{1,3,5}$, Bradley T. Webb ${ }^{6,1}$, Brien P. Riley ${ }^{1,3,5}$

${ }^{1}$ Virginia Institute for Psychiatric and Behavioral Genetics, Virginia Commonwealth University, Richmond, Virginia, USA

${ }^{2}$ Integrative Life Sciences Doctoral Program, Virginia Commonwealth University, Richmond, Virginia, USA

${ }^{3}$ Department of Psychiatry, Virginia Commonwealth University, Richmond, Virginia, USA

${ }^{4}$ Center for the Study of Biological Complexity, Virginia Commonwealth University, Richmond, Virginia, USA

${ }^{5}$ Department of Human and Molecular Genetics, Virginia Commonwealth University, Richmond, Virginia, USA

${ }^{6}$ GenOmics, Bioinformatics, and Translational Research Center, Biostatistics and Epidemiology Division, RTI International, Research Triangle Park, NC, USA

\section{Keywords Schizophrenia, PRS, Multiplex Families}

Multiplex schizophrenia families show higher recurrence risk compared to the families of singleton cases, but the source of increased familial recurrence risk is unknown. Two possible hypotheses to explain this observation are (1) a higher burden of common risk variants in the families, or (2) segregation of rare high risk variants in the families. We sought to test the first hypothesis in a large, ethnically homogenous sample of multiplex schizophrenia families, singleton cases and controls, all from the population of Ireland. Polygenic risk scores (PRS) based on the 2nd and 3rd Psychiatric Genomics Consortium (PGC) mega-analyses of schizophrenia were constructed for multiplex family members $(\mathrm{N}=1005)$, singleton cases $(\mathrm{N}=2224)$, and controls $(\mathrm{N}=2284)$ and analyses were conducted using mixed effects logistic regression which accounts for the family structure as a random effect. The schizophrenia PRS in familial cases did not differ significantly from singleton cases $(p=0.82$ and $p=0.32$ for PGC2 and PGC3, respectively). We also show that the unaffected family members have a significantly higher PRS compared to population controls ( $p<0.001$ for PGC2 and PGC3), indicating the presence of a higher burden of common schizophrenia risk variants across family members. These results suggest that a higher burden of common risk variants is unlikely to account for the increased recurrence risk of schizophrenia in multiplex families. In the absence of an elevated PRS in family cases, segregation of rarer variation in the genome, as identified through whole-genome sequencing may explain part of the higher recurrence risk of schizophrenia in multiplex families.

Grant Support: National Institute of Mental Health grant R01MH114593 (to BPR).
Influence of the Fetal Genome on Mothers' Emotional Symptoms During Pregnancy: Exploring the Possibility of Prenatal child-to-Parent Effects

Yasmin I. Ahmadzadeh ${ }^{1}$, Rosa C. Cheesman ${ }^{1,2}$, Daniel Wechsler ${ }^{1}$, Isabella Badini $^{1}$, Eivind Ystrom ${ }^{2,3,4}$, Espen M. Eilertsen ${ }^{2,5}$,

Tom A. McAdams ${ }^{1,2}$

${ }^{1}$ Social, Genetic and Developmental Psychiatry Centre, Institute of Psychiatry, Psychology and Neuroscience, King's College, London, UK

${ }^{2}$ PROMENTA Research Center, Department of Psychology, University of Oslo, Oslo, Norway

${ }^{3}$ Pharmaco Epidemiology and Drug Safety Research Group, School of Pharmacy, University of Oslo, Oslo, Norway

${ }^{4}$ Department of Mental Disorders, Norwegian Institute of Public Health, Oslo, Norway

${ }^{5}$ Centre for Fertility and Health, Norwegian Institute of Public Health, Oslo, Norway

Keywords Prenatal, Anxiety, Depression, Genetics, MoBa

There is abundant literature on the possible causal effects of mothers' emotional symptoms during pregnancy on child development. To our knowledge, there has been no investigation into the reverse possibility, whereby child (i.e., fetal) factors exert causal influence on mothers' prenatal emotional symptoms. What is more, there is no knowledge of the total paternal indirect genetic effect on maternal depression during pregnancy. If fathers exert influence on mother's prenatal symptoms, then mother-child associations are confounded by paternal effects. Parent and child genetic effects on mothers' prenatal symptoms can be differentiated using SNP-based variance decomposition methods, for example trio-genome complex trait analysis (Trio-GCTA) (Eilertsen et al., 2021). We explore this using data from the Norwegian Mother, Father and Child Cohort Study (MoBa). In the MoBa, mothers' symptoms of anxiety and depression were measured using the Hopkins Symptom Checklist at 15- and 30-weeks gestation, and genotype data are available for approximately 25,000 parentoffspring trios. Preliminary findings will be presented, alongside a discussion of method triangulation using extended pedigree structures in the MoBa.

\section{References}

Eilertsen, E.M., Jami, E.S., McAdams, T.A. et al. Direct and Indirect Effects of Maternal, Paternal, and Offspring Genotypes: Trio-GCTA. Behav Genet 51, 154-161 (2021).

Grant Support: TM and YA are supported by a Wellcome Trust Senior Research Fellowship awarded to TM (220382/Z/20/Z). DLW is supported by the UK Medical Research Council (MR/N013700/1) and King's College London member of the MRC Doctoral Training Partnership in Biomedical Sciences. IB is supported by the UK Economic and Social Research Council (ESRC) and King's College London member of the ESRC Doctoral Training Partnership in Interdisciplinary Social Science (LISS DTP). RC (288083), EY (262177; 288083), and TM (288083) are supported by the Research Council of Norway. The MoBa data collection was supported by the Norwegian Ministry of Health and Care Services and the Ministry of Education and Research, NIH/NIEHS (contract N01-ES-75558), NIH/ NINDS (grants 1 UO1 NS 047537-01, 2 UO1 NS 047537-06A1). 


\section{Individual Differences in Daily Mobility Patterns in a Genetically Informed Youth Sample}

\author{
Jordan D. Alexander ${ }^{1}$, Yuan Zhou, Samantha M. Freis ${ }^{2,3}$, \\ Naomi P. Friedman ${ }^{2,3}$, \& Scott I. Vrieze ${ }^{1}$ \\ ${ }^{1}$ Department of Psychology, University of Minnesota, Minneapolis, \\ Minnesota, USA \\ ${ }^{2}$ Institute for Behavioral Genetics, University of Colorado Boulder \\ ${ }^{3}$ Department of Psychology and Neuroscience, University of Color- \\ ado Boulder
}

Keywords GPS Location Data, Personality, Emerging Adulthood, ACE Decomposition

Daily mobility patterns (DMP) extracted from GPS location data offer a standardized objective tool for environmental measurement. Though these measures are increasingly prevalent in psychological research, key questions remain regarding sources of individual differences in DMP, as well as their developmental course and heritability. Using persistent smartphone location data collected from June, 2016 through December, 2019 ( $\mathrm{N}=13.2$ million locations) from 709 Coloradobased adolescent twins, we investigated the development and genetic and environmental contributions to DMP during adolescence and emerging adulthood. Additionally, we examined whether individual differences were partly explained by personality, as measured by the big five personality inventory. Mixed effects model results suggested that, though there were large individual differences, mobility generally increased throughout adolescence before decreasing slightly from age 18-21. Multivariate ACE models identified moderate genetic and shared environmental components to daily locations visited and entropy of movement $(\mathrm{A}=0.42 ; 0.37, \mathrm{C}=0.32 ; 0.35)$ while genetic factors largely accounted for daily distance travelled $(\mathrm{A}=0.74)$. Finally, ACE Models applied to random age effects identified complex changes in DMP heritability patterns over time: heritability generally increased early in adolescence, remained constant in late adolescence, and declined in emerging adulthood. Regarding personality, DMP were positively associated with extraversion and conscientiousness and negatively associated with openness. Genetic correlations revealed relationships between extraversion and DMP were partially explained by shared genetic effects $(\mathrm{r}=0.14-0.25 ; r G=$ $0.25-0.53$ ). Results suggest large individual differences in the developmental course of DMP which are partially accounted for by genes, shared environments, and personality.

Grant Support: National Institute on Drug Abuse award U01 DA046413.

\section{Intergenerational Transmission of BMI and Educational Attainment}

\author{
Hekmat Alrouh ${ }^{1,2}$, Elsje van Bergen ${ }^{1,2}$, Conor V. Dolan ${ }^{1,2}$, \\ Dorret I. Boomsma ${ }^{1,2}$ \\ ${ }^{1}$ Department of Biological Psychology, Faculty of Behavioral and \\ Movement Sciences, Vrije Universiteit Amsterdam, Amsterdam, \\ Netherlands \\ ${ }^{2}$ Amsterdam Public Health research institute, Amsterdam, \\ Netherlands
}

Keywords Intergenerational Transmission, Educational Attainment, Body Mass Index, Spousal Correlation, Structural Equation Modeling Individual differences in educational attainment (EA) and body mass index (BMI) are correlated and are subject to intergenerational transmission ${ }^{1}$. The aim of our study is to address the question as to what extent BMI is transmitted from one generation to the next, and what the role of EA is in this transmission. Our study population is a sample of 42,413 young twins from the Netherlands Twin Register (NTR). First, we will simultaneously analyze direct (i.e., within trait) and indirect (i.e., across trait) transmission of BMI and EA by structural equation modeling (SEM). Second, we will apply a nuclear twin family design ${ }^{2}$ to test for genetic and cultural transmission of BMI from parents to children. Both analyses will be performed on data from early childhood (5 years old) and adolescence (12 years old) to analyze differences in the roles of genetic and environmental factors in the transmission of BMI at those two life stages. We will contrast our findings of the SEM portion of the analyses in our young sample to the results we previously obtained in adult offspring and their parents, where we found strong evidence for intergenerational transmission of EA and BMI, and significant within- and cross-trait correlations both within person and between spouses ${ }^{3}$.

\section{References}

${ }^{1}$ Newton, S., Braithwaite, D., \& Akinyemiju, T. F. (2017). Socioeconomic status over the life course and obesity: Systematic review and meta-analysis. PloS one, 12(5), e0177151.

${ }^{2}$ Keller, M. C., Medland, S. E., Duncan, L. E., Hatemi, P. K., Neale, M. C., Maes, H. H., \& Eaves, L. J. (2009). Modeling extended twin family data I: description of the Cascade model. Twin research and human genetics, 12(1), 8-18.

${ }^{3}$ Alrouh H., van Bergen E., de Zeeuw E., Dolan C., \& Boomsma D. I. (2021) Intergenerational Transmission of BMI and Educational Attainment in Adults. Manuscript submitted for publication.

Grant Support: NWO Hestia: The impact of parental genes on offspring health: nurture via nature. (VidW.1154.19.013); NWO Veni (451-15-017); NWO Consortium on Individual Development (024.001.003).

\section{Genetic and Environmental Structure of Altruism Characterized by Recipients in Relation to Big Five Personality}

Juko Ando ${ }^{1} \&$ Tetsuya Kawamoto ${ }^{2}$

${ }^{1}$ Faculty of Letters, Keio University, Tokyo, Japan

${ }^{2}$ Faculty of Letters, Kokushikan University, Tokyo, Japan

Keywords Altruism, Kin selection, Direct reciprocity, Indirect reciprocity, Big five personality

Altruism is a form of prosocial behavior with the goal of increasing the fitness of another individual as a recipient while reducing the fitness of the actor. Although there are many studies on its heterogeneity, only a few behavioral genetic studies have been conducted to examine the differential recipient types: family members favored by kin selection, the dynamic network of friends and acquaintances as direct reciprocity, and strangers as indirect reciprocity. The present study shows that there is a single common factor of altruism: additivegenetic effects explain $51 \%$ of altruism without shared environmental contribution. The genetic contribution of this single common factor is the genetic factor of neuroticism, extraversion, openness to experience, and conscientiousness. Only altruism toward strangers is affected by shared environmental factors. Genetic and environmental relationship between a general factor of altruism (GFA) and a general factor of personality (GFP) is examined.

Grant Support: Topic-Setting Program to Advanced Cutting-Edge Humanities and Social Sciences Research (FY2013-FY2015). 


\section{Stressful Life Events and Borderline Personality Disorder Traits}

\author{
Vilde Sofie Arneberg ${ }^{1}$, Vilde Fodstad ${ }^{1}$, Eivind Ystrom ${ }^{1,2}$ \\ ${ }^{1}$ Department of Psychology, University of Oslo, Oslo, Norway \\ ${ }^{2}$ Department of Mental Disorders, Norwegian Institute of Public \\ Health, Oslo, Norway
}

Keywords Personality Disorders, Life Events, Stress, Elastic Net, Trauma

Borderline personality disorder is a mental disorder linked to considerable impairment. Multiple studies have found frequent traumatic experiences in childhood among BPD patients, and we therefor address the following problems: One, what types of SLE are associated with borderline personality disorder traits? Two, to what extent is the association between the combined SLEs and BPD accounted for by familial genetic and environmental background factors? In total 2801 twins participated from the Norwegian Institute of Public Health (NIPH) Twin Panel. Negative binomial Poisson regression was used to explore which SLEs could predict BPD traits. An Elastic Net penalized regression analysis with a subsequent biometric co-twin control was performed to develop the final logistic regression model for prediction and to differentiate between environmental and genetic factors in our sample. We found that in childhood, life-threatening experiences and an unpredictable and unsafe environment is associated with the development of BPD traits. In adulthood, lifethreatening experiences, economic issues, and relationship conflicts had the same association. Our results indicate that these experiences predict $22 \%$ of the risk for BPD traits. In the adjusted model, $41.7 \%$ of shared environmental, $16.7 \%$ of additive genetic, and $5.9 \%$ of individual-specific environmental effects for BPD traits could be explained by SLEs. In the debate about how stressful life events are associated with the development of BPD traits, our results point to the shared family environment as a common cause for both the SLEs and the development of BPD traits.

Grant Support: VS Arneberg and V Fodstad are supported by a Research Council of Norway Student Grant. E Ystrom is supported by the Research Council of Norway (262177 and 288083). The data collection was funded by the Research Council of Norway (196148).

\section{Heritability of Infant Traits: Meta-analysis of Twin Studies of Psychological Traits and Developmental Milestones in Infancy}

Chloe Austerberry ${ }^{1}$, Maria Mateen ${ }^{1}$, Pasco Fearon ${ }^{1}$, Angelica Ronald ${ }^{2}$ ${ }^{1}$ Department of Clinical, Educational and Health Psychology, UCL, London, UK

${ }^{2}$ Department of Psychological Sciences, Birkbeck, University of London, London, UK

Keywords Heritability, Twin, Meta-analysis, Infancy

Despite the importance of infancy as a period of rapid postnatal growth and development, the causes of variation in infant traits remain ambiguous as no one has synthesized findings on genetic and environmental influences on infant phenotypes. We systematically retrieved and extracted findings from 139 papers from 52 twin studies using the classical twin design in infants aged between 0 and 24 months (PROSPERO protocol registration CRD42019151532). Findings included 377 psychological and developmental phenotypes from 78,421 pairs (30,732 MZ; 47,689 DZ), in 21 different countries. We manually classified findings using the WHO International Classification of Functioning, Disability and Health for Children and Youth (ICF-CY) and conducted three-level multilevel random-effects meta-analyses of twin correlations for phenotypes from 10 ICF-CY categories. These models incorporated sampling variance, withincohort variance in outcome measurement and between-cohort variance, allowing multiple findings to be included from each twin study and multiple different measures of a phenotype. Heritability and shared and nonshared environmental estimates were calculated in meta-analytic SEM models using the correlations and variances from the multilevel meta-analyses. Results provided consistent evidence that psychological traits and developmental milestones are heritable from as early as $0-2$ years and are influenced by the shared and non-shared environment. Phenotypic domains with the highest heritabilities were psychomotor functions and attention and those with the highest shared environmental estimates were language, basic cognitive functions and sleep functions. This first meta-analysis of infant traits confirms significant modest twin heritability across a range of infant traits and milestones.

Grant Support: Chloe Austerberry was supported by a UK Economic and Social Research Council (ESRC) Studentship awarded by the UCL, Bloomsbury and East London Doctoral Training Partnership (ES/P000592/1).

\section{Polygenic p: Characterizing the Role of General Genetic Liability to Psychopathology in Maternal Depression}

Ziada Ayorech ${ }^{1,2,3}$, Rosa Cheesman ${ }^{1}$, Espen M. Eilertsen ${ }^{1}$, Alexandra Havdahl ${ }^{1,2,3}$, Eivind Ystrom ${ }^{1,3,4}$

${ }^{1}$ PROMENTA Research Center, Department of Psychology, University of Oslo, Norway

${ }^{2} \mathrm{Nic}$ Waals Institute, Oslo, Norway

${ }^{3}$ Department of Mental Disorders, Norwegian Institute of Public Health, Oslo, Norway

${ }^{4}$ School of Pharmacy, University of Oslo, Norway

Keywords Maternal depression, Fathers, Polygenic scores, p factor, $\mathrm{MoBa}$

Maternal depression (MD) is associated with a number of severe consequences for mother's physical and emotional wellbeing, including suicide. In cases of MD, approximately $24-50 \%$ of partners also develop depression. Despite evidence for substantial genetic contribution to its liability, few MD variants have been identified, especially in contrast to other psychiatric disorders and behavioural traits. We exploit recent evidence of a general dimension $(p)$ capturing liability to psychopathology to calculate 'polygenic $p$ ', the first principal component of ten major psychiatric disorder polygenic scores, in over 25,000 mothers and fathers, participating in the Norwegian Mother Father and Child Cohort Study (MoBa). Polygenic p is used to predict lifetime history of depression in both mothers and fathers as well as development of depression during the first decade of parenthood (10 assessments from gestational age 17 weeks to 8 years). Results could boost genetic prediction of depression symptom risk and resilience and provide new evidence of genetic assortative mating for psychopathology.

Grant Support: ZA is funded by a Marie Skłodowska-Curie Fellowship from the European Union (894675). RC and AH (288083) and EY $(288083 ; 262177)$ are supported by the Research Council of Norway. 


\section{Socioeconomic Status and Risk for Child Emotional and Behavioral Problems: Exploring Gene- Environment Interaction in the Presence of Gene- Environment Correlation Using Extended Families in the Norwegian Mother, Father and Child Birth Cohort Study}

Isabella Badini ${ }^{1}$, Torkild H. Lyngstad ${ }^{2}$, Espen M. Eilersten ${ }^{3}$, Yasmin I Ahmadzadeh ${ }^{1}$, Daniel L Wechsler ${ }^{1}$,

Henrik Daae Zachrisson ${ }^{4,5}$, Nicolai T. Borgen ${ }^{5}$, Helena Zavos ${ }^{6}$, Eivind Ystrom $^{3,7,8}$, Tom A. McAdams ${ }^{1,7}$

${ }^{1}$ Social, Genetic, \& Developmental Psychiatry Centre, Institute of Psychiatry, Psychology and Neuroscience, King's College London, London, United Kingdom

${ }^{2}$ Department of Sociology and Human Geography, University of Oslo, Oslo, Norway

${ }^{3}$ Norwegian Institute of Public Health, Oslo, Norway

${ }^{4}$ The Norwegian Center for Child Behavioral Development, Oslo, Norway

${ }^{5}$ Department of Special Needs Education, University of Oslo, Oslo, Norway

${ }^{6}$ Department of Psychology, Institute of Psychiatry, Psychology and Neuroscience, King's College London, London, United Kingdom

${ }^{7}$ PROMENTA Research Center, University of Oslo, Oslo, Norway

${ }^{8}$ PharmacoEpidemiology and Drug Safety Research Group, School of Pharmacy, University of Oslo, Oslo, Norway

Keywords Childhood behavior problems, Socioeconomic status, Gene-environment interaction, Gene-environment correlation

Socioeconomic disadvantage is associated with increased risk for child emotional and behavioral problems. There is some evidence from twin studies that family socioeconomic status (SES) moderates the relative contribution of genetic and environmental influences on child mental health. In the current study, we applied a novel approach using extended family data comprising twin, sibling, and half-sibling pairs of parents and their children from The Norwegian Mother and Child Cohort Study (MoBa) to test for gene-environment interaction in the presence of gene-environment correlation. The Multiple-Children-of-Twins-and-Siblings (MCoTS) design was adapted to investigate whether etiological influences on child emotional and behavioral problems vary as a function of family SES. National administrative register data on maternal and paternal income rank and educational attainment was used to index parent SES. Child emotional and behavioral symptoms were assessed at ages 1.5-5 years. Results indicated greater variance in child emotional and behavioral problems in families with low parental income and education. Etiological influences on child emotional and behavioral problems were significantly moderated by maternal SES. The etiology of child emotional, but not behavioral, problems was found to be significantly moderated by paternal SES. Findings offer additional insights into the role that family SES plays in shaping the etiology of early childhood emotional and behavioral problems.

Grant Support: IB is supported by the UK Economic and Social Research Council (ESRC) and King's College London member of the ESRC Doctoral Training Partnership in Interdisciplinary Social Science (ST11872). TAM and YIA are supported by a Wellcome Trust Senior Research Fellowship awarded to TAM (220382/Z/20/Z). DLW is supported by the UK Medical Research Council (MR/N013700/1) and King's College London member of the MRC Doctoral Training Partnership in Biomedical Sciences. THL is supported by a Consolidator grant from the European Research Council (ERC) under the Horizon 2020 research and innovation programme (Grant agreement
No. 818420). NTB is supported by a ESRC grant (\#818425). The MoBa data collection was supported by the Norwegian Ministry of Health and Care Services and the Ministry of Education and Research, NIH/NIEHS (contract N01-ES-75558), NIH/NINDS (grants 1 UO1 NS 047537-01, 2 UO1 NS 047537-06A1).

\section{Bivariate Estimation of Vertical Transmission and Genetic Nurture Using Polygenic Scores}

Jared V. Balbona ${ }^{1,2}$, Yongkang $\mathrm{Kim}^{1}$, and Matthew C. Keller ${ }^{1,2}$

${ }^{1}$ Institute of Behavioral Genetics, University of Colorado Boulder, Boulder, Colorado, USA

${ }^{2}$ Department of Psychology and Neuroscience, University of Colorado Boulder, Boulder, Colorado, USA

Keywords Vertical Transmission, Genetic Nurture, Heritability Estimation, Structural Equation Modeling

As offspring resemble their parents for both genetic and non-genetic reasons, family-based approaches have struggled to disentangle variance due to shared genetic factors from variance due to shared environmental factors. Several recent studies ${ }^{1,2}$ have sought to differentiate these overlapping causes by examining the extent to which parents' non-transmitted alleles influence their offspring's phenotype; however, no studies to date have been able to estimate the effect of a parental trait on a different offspring trait without suffering significant bias, thus greatly limiting the number of questions that can be asked using these approaches. Here, we introduce a series of bivariate causal models that address this gap by using parental polygenic scores (PGS's) to obtain within- and cross-trait estimates of both vertical transmission and genetic nurture. Unlike past approaches, these models are able to (1) account for direct genetic effects, pleiotropy between PGS's, and within- and cross-trait assortative mating at both equilibrium and disequilibrium; (2) provide unbiased estimates of vertical transmission and genetic nurture, even when a trait's PGS explains only a small portion of total heritability; (3) be implemented without full trio data; and (4) be easily altered or extended to best fit the traits and data being used. As such, these models provide a novel approach for understanding how parental genes and traits influence their offspring.

\section{References}

${ }^{1}$ Kong A, Thorleifsson G, ..., Gudbjartsson DF (2018). The nature of nurture: Effects of parental genotypes. Science, 359(6374),424-428.

${ }^{2}$ Balbona JV, Kim Y, Keller MC (2021). Estimation of parental effects using polygenic scores. Behavior Genetics.

Grant Support: MCK is supported by National Institute of Mental Health grant R01 MH100141.

\section{Adverse Childhood Experiences and Mental Health: A Genetically Informed Study}

Jessie R. Baldwin ${ }^{1,2}$, Hannah M. Sallis ${ }^{3,4,5,6}$, Tabea Schoeler ${ }^{1}$, Mark J. Taylor ${ }^{7}$, Alex S. F. Kwong ${ }^{3,8}$, Laura D. Howe ${ }^{3}$, Andrea Danese ${ }^{2,9,10}$, Eamon McCrory ${ }^{1,11}$, Fruhling Rijsdijk ${ }^{2}$, Jorim J. Tielbeek $^{12}$, Wikus Barkhuizen ${ }^{1}$, Henrik Larsson ${ }^{7,13}$, Sebastian Lundström ${ }^{14,15}$, Robert Karlsson ${ }^{7}$, Paul Lichtenstein ${ }^{7}$, Marcus Munafó $\grave{j}^{3,4,5}$, \& Jean-Baptiste Pingault ${ }^{1,2}$ 
${ }^{1}$ Department of Clinical, Educational and Health Psychology, Division of Psychology and Language Sciences, University College London, London, UK

${ }^{2}$ Social, Genetic and Developmental Psychiatry Centre, Institute of Psychiatry, Psychology and Neuroscience, King's College London, London, UK

${ }^{3}$ MRC Integrative Epidemiology Unit at the University of Bristol, Bristol Medical School, University of Bristol, Bristol, UK

${ }^{4}$ School of Psychological Science, University of Bristol, Bristol, UK ${ }^{5}$ NIHR Biomedical Research Centre, University Hospitals Bristol NHS Foundation Trust and University of Bristol, Bristol, UK

${ }^{6}$ Centre for Academic Mental Health, Population Health Sciences, Bristol Medical School, University of Bristol, Bristol, UK

${ }^{7}$ Department of Medical Epidemiology and Biostatistics, Karolinska Institutet, Nobels väg 12A, 17177 Stockholm, Sweden

${ }^{8}$ Division of Psychiatry, Edinburgh Medical School, University of Edinburgh, Edinburgh, EH10 5HF, UK

${ }^{9}$ Department of Child \& Adolescent Psychiatry, Institute of Psychiatry, Psychology \& Neuroscience, King's College London, London SE5 8AF, United Kingdom

${ }^{10}$ National and Specialist CAMHS Trauma, Anxiety, and Depression Clinic, South London and Maudsley NHS Foundation Trust, London, UK

${ }^{11}$ Anna Freud National Centre for Children and Families, London, UK

${ }^{12} \mathrm{CNCR}$, Amsterdam Neuroscience Campus, VU University, Amsterdam, The Netherlands

${ }^{13}$ School of Medical Sciences, Örebro University, Örebro, Sweden

${ }^{14}$ Gillberg Neuropsychiatry Centre, Institute of Neuroscience and Physiology, University of Gothenburg, Gothenburg, Sweden

${ }^{15}$ Centre for Ethics, Law and Mental Health (CELAM), Institute of Neuroscience and Physiology, University of Gothenburg, Gothenburg, Sweden

Keywords Childhood adversity, Mental health, Gene-environment correlation, Genetic confounding, Polygenic scores

Children exposed to adverse childhood experiences (ACEs) have an elevated risk of mental health problems, but it is unclear whether these associations reflect genetic confounding. We tested (1) whether children with genetic liability to psychopathology are more likely to experience ACEs, and (2) the extent to which the associations between ACEs and mental health are genetically confounded. Participants were 6411 children from the Avon Longitudinal Study of Parents and Children (ALSPAC). ACEs (including maltreatment, domestic violence, and parental psychopathology, substance abuse, criminality, and separation) were prospectively measured through parent reports at multiple assessments between birth and age 9 . Internalizing and externalizing problems at age 9 were assessed through parent reports on the Development and Wellbeing Assessment. We derived polygenic scores for a range of psychiatric disorders. Children with greater genetic liability to psychopathology had a small elevation in risk of ACEs (pooled odds ratio $=1.05,95 \%$ CI 1.01-1.09). Measured polygenic scores accounted for a very small proportion of the associations between ACEs with internalizing problems (pooled average across ACEs $=3.6 \%$ ) and externalizing problems (pooled average $=4.8 \%$ ). However, latent polygenic scores capturing SNP heritability in mental health outcomes explained a larger proportion of the associations between ACEs with internalizing problems (pooled average $=63 \%$ ) and externalizing problems (pooled average $=17 \%$ ). Risk of mental health problems in children exposed to ACEs is partly, but not completely driven by pre-existing genetic liability to psychopathology. Assuming the absence of nongenetic confounding, these findings are consistent with a partly causal effect of ACEs on mental health.

Grant Support: Wellcome Trust grant 215917/Z/19/Z.

\section{The Effect of Education on the Relationship between Genetics, Early-Life Disadvantages, and Later-Life SES}

Silvia H. Barcellos ${ }^{1,2,3}$, Leandro S. Carvalho ${ }^{1,2}$, and Patrick Turley ${ }^{1,2}$ ${ }^{1}$ Department of Economics, University of Southern California, Los Angeles, California, USA

${ }^{2}$ Center for Economic and Social Research, University of Southern California, Los Angeles, California, USA

${ }^{3}$ National Bureau of Economic Research, Cambridge, Massachusetts, USA

Keywords Gene-by-environment, Polygenic index, Family study, Education, Socioeconomic status

This paper investigates whether education weakens the relationship between early-life disadvantages and later-life SES. We use three proxies for advantage that we show are independently associated with SES in middle-age. Besides early, favorable family and neighborhood conditions, we argue that the genes a child inherits also represent a source of advantages. Using a regression discontinuity design and data for over 110,000 individuals, we study a compulsory schooling reform in the UK that generated exogenous variation in schooling. While the reform succeeded in reducing educational disparities, it did not weaken the relationship between early-life disadvantages and wages. This implies that advantaged children had higher returns to schooling. We exploit family-based random genetic variation and find no evidence that these higher returns were driven by geneticallyinfluenced individual characteristics such as innate ability or skills.

Grant Support: Research reported in this publication was supported through the Russell Sage Foundation grant 98-16-16, NIA grants K99-AG062787-01, K01AG050811, R21AG060447, RF1AG055654, 3RF1AG055654-01A1S1, and R56AG058726, Open Philanthropy grant 010623-00001, and by the USC Population Research Center.

\section{Direct and Indirect Genetic Influences Involved in the Intergenerational Transmission of Risk for ADHD in the Norwegian Mother, Father and Child Cohort}

Wikus Barkhuizen ${ }^{1 *}$, Jean-Baptiste Pingault ${ }^{1,2}$, Biyao Wang $^{1}$, Laurie J. Hannigan ${ }^{3,4}$, Espen Moen Eilertsen ${ }^{6}$, Ole A. Andreassen ${ }^{7}$, Helga Ask ${ }^{5}$, Martin Tesli ${ }^{5,7}$, Ragna Bugge Askeland ${ }^{4,5}$,

George Davey Smith ${ }^{4,9}$, Neil Davies ${ }^{4,8,9}$, Ted Reichborn-Kjennerud ${ }^{5}{ }^{\dagger}$ Eivind Ystrom ${ }^{5,6,10}{ }^{\dagger}$ Alexandra Havdahl ${ }^{3,5,6}$

*Equal first authors ${ }^{\dagger}$ Equal last authors.

${ }^{1}$ Division of Psychology and Language Sciences, University College London, London, United Kingdom

${ }^{2}$ MRC Social, Genetic and Developmental Psychiatry Centre, Institute of Psychiatry, King's College, London, United Kingdom

${ }^{3} \mathrm{Nic}$ Waals Institute, Lovisenberg Diaconal Hospital, Oslo, Norway ${ }^{4}$ Medical Research Council Integrative Epidemiology Unit, University of Bristol, United Kingdom

${ }^{5}$ Department of Mental Disorders, Norwegian Institute of Public Health, Oslo, Norway

${ }^{6}$ PROMENTA Research Center, Department of Psychology, University of Oslo, Oslo, Norway

${ }^{7}$ NORMENT Centre, Institute of Clinical Medicine, University of Oslo and Division of Mental Health and Addiction, Oslo University Hospital, Oslo, Norway 
${ }^{8}$ K.G. Jebsen Center for Genetic Epidemiology, Department of Public Health and Nursing, NTNU, Norwegian University of Science and Technology, Norway

${ }^{9}$ Population Health Sciences, Bristol Medical School, University of Bristol, Barley House, Oakfield Grove, Bristol, United Kingdom

${ }^{10}$ School of Pharmacy, University of Oslo, Oslo, Norway

Keywords MoBa, Attention-deficit/hyperactivity disorder, Intergenerational transmission, Genetic nurture, Parenting

Direct genetic transmission of risk may partly account for epidemiological associations between parental risk factors and children's attention-deficit/hyperactivity disorder (ADHD) symptoms. Indirect genetic influences may also contribute to children's ADHD symptoms via mechanisms such as genetically influenced parenting environments which in turn contributes to ADHD liability in children (also called "genetic nurture"), population stratification or assortative mating. Our study sample consisted of 5405 family trios from the Norwegian Mother, Father and Child Cohort Study (MoBa). Mothers reported on their 8-year-old children's ADHD symptoms using the Parent/Teacher Rating Scale for Disruptive Behavior Disorders. We computed polygenic scores for parental traits previously associated with ADHD, including psychopathology, substance use, neuroticism, educational attainment and intellectual ability. Jointly modelling polygenic scores for fathers, mothers and children to statistically control for genetic transmission, children's polygenic scores for ADHD ( $\beta=0.10 ; 95 \%$ CI 0.07 to 0.14 ), smoking ( $\beta=0.07 ; 95 \%$ CI 0.03 to 0.10$)$ and educational attainment $(\beta=-0.09 ; 95 \% \mathrm{CI}-0.13$ to -0.05$)$ predicted ADHD symptoms, suggesting direct genetic effects. Mothers' polygenic scores for autism spectrum disorder $(\beta=$ $0.05 ; 95 \%$ CI 0.02 to 0.08 ) and neuroticism ( $\beta=0.05 ; 95 \%$ CI 0.01 to 0.08 ) predicted children's ADHD symptoms, implicating indirect genetic effects. Both direct genetic transmission and genetic nurture likely contribute to associations between some parental traits and children's ADHD symptoms. These findings highlight the importance of considering the role of genetic influences when investigating intergenerational transmission of risk of ADHD

Grant Support: The Research Council of Norway supported EME (\#262177), EY (\#262177 and \#288083), TRK, HA and RBA (\#274611) and AH (\#288083). AH and LJH was supported by the South-Eastern Norway Regional Health Authority (2020022 and 2018058, respectively). JBP is supported by the Medical Research Foundation 2018 Emerging Leaders 1st Prize in Adolescent Mental Health (MRF-160-0002-ELP-PINGA). GDS is supported by the Medical Research Council (MRC) and the University of Bristol [MC_UU_00011/1]. This project has received funding from the European Research Council (ERC) under the European Union's Horizon 2020 research and innovation programme (grant agreement No. 863981). The Medical Research Council (MRC) and the University of Bristol support the MRC Integrative Epidemiology Unit [MC_UU_00011/1]. NMD is supported by a Norwegian Research Council Grant number 295989.

\section{Sex Differences in Heritability-By-Age of Latent Dementia Risk in 10 Studies of the Consortium on Interplay of Genes and Environment Across Multiple Studies}

Christopher R. Beam ${ }^{1}$, Susan E. Luczak ${ }^{1}$, Chandra A. Reynolds ${ }^{2}$, Matthew Panizzon ${ }^{3}$, Kayla Tureson ${ }^{1}$, Margaret Gatz ${ }^{1,4}$ The Consortium on Interplay of Genes and Environment Across Multiple Studies
${ }^{1}$ Department of Psychology, University of Southern California, Los Angeles, California, USA

${ }^{2}$ Department of Psychology, University of California - Riverside, Riverside, CA, USA

${ }^{3}$ Department of Psychiatry, Center for Behavior Genetics of Aging, University of California San Diego, La Jolla, CA

${ }^{4}$ Center for Economic and Social Research, University of Southern California, Los Angeles, California, USA

Keywords Dementia risk, Heritability, Sex differences, Lifespan development

Genetically informed studies of dementia are rare with heritability of dementia estimated at approximately $60 \%$ across older adulthood. Alzheimer's disease researchers have suggested that, within older adults, earlier age of onset might be associated with greater genetic influence. The ordinal nature of dementia diagnosis outcomes severely affects power to test for age differences in heritability, as well as to detect significant sex differences in heritability estimates. The first aim of the current study is to examine age differences in the heritability of dementia likelihood across older adulthood. The second aim is to test for sex differences in these patterns. The current study uses 7568 pairs of twins across 10 studies from The Consortium on Interplay of Genes and Environment Across Multiple Studies (IGEMS). Dementia likelihood is quantified continuously using a latent dementia index measure that is estimated from twins' available cognitive, memory-specific, and functional ability measures. Quadratic age-trends are estimated for men and women using a modified twin correlation model (Turkheimer et al. 2017). Significant quadratic effects of age were observed in women but not men. Heritability increases in men from approximately $16 \%$ at age 60 years to greater than $80 \%$ by age 100 years. However, heritability in women decreases with age, with estimates as high as $64 \%$ at age 60 years and $10 \%$ by age 100 years. The genetic basis for dementia likelihood, thus, appears to vary drastically between men and women across older adulthood.

\section{References}

Turkheimer E, Beam CR, Sundet, JM, \& Tambs, K. (2017). Interaction between parental education and twin correlations for cognitive ability in a Norwegian conscript sample. Beh gen, 47, 507-515.

Grant Support: NIH/NIA Grants R01 AG060470 and RF1 AG058068.

\section{Preliminary Results of Cognitive Ability Trajectories from Infancy Through Middle-Age in the Louisville Twin Study}

Christopher R Beam ${ }^{1,2}$, Deborah Finkel ${ }^{3,4}$, Eric Turkheimer ${ }^{5}$, Thomas Guterbock $^{6}$, Sean Johnson ${ }^{6}$, Evan J. Giangrande ${ }^{5}$, Natalie Pasquenza ${ }^{7}$, Lesa Ryan $^{7}$, Deborah W. Davis ${ }^{7}$

${ }^{1}$ Department of Psychology, University of Southern California, Los Angeles, California, USA

${ }^{2}$ School of Gerontology, University of Southern California, Los Angeles, California, USA

${ }^{3}$ Department of Psychology, Indiana University Southeast, New Albany, Indiana, USA

${ }^{4}$ Institute for Gerontology, Jönköping University, Småland, Sweden

${ }^{5}$ Department of Psychology, University of Virginia, Charlottesville, Virginia, USA 
${ }^{6}$ Center for Survey Research, University of Virginia, Charlottesville, Virginia, USA

${ }^{7}$ Department of Pediatrics, University of Louisville, Louisville, Kentucky, US

Keywords Cognitive ability, Lifespan development, Louisville Twin Study

After a 20-year study hiatus, data collection in the Louisville Twin Study is again underway. As many of the twins are now middle-aged (> 40 years), current study of the LTS twins focuses on physical and cognitive aging, with a special focus on childhood and midlife risk factors of preclinical symptoms of Alzheimer's disease. Chief among these is clarifying the genetic and environmental processes through which childhood and adolescent cognitive development contributes to midlife cognitive functioning. In 2020, the data collection of the middle life phase of the Louisville Twin Study began in earnest, despite a hindered start due to the COVID-19 pandemic. To date, we have collected data on 43 twins (14 complete pairs) out of the proposed target sample of 750 individual twins. We expect study visits to increase as COVID-19 quarantine and social distancing restrictions are eased in 2021 and 2022. The purpose of this talk is to present preliminary results on the correlations between Full Scale IQ (FSIQ) scores at ages $6,7,8,9,12$, and 15 with scores at midlife. Mean age at study visit was $55.21(S D=3.64)$ and midlife age standardized FSIQ is 107.37 ( $S D=16.11)$. Medium $(r=.53)$ to large $(r=.83)$ correlations were observed between childhood and midlife FSIQ scores, with age 8 , age 9 and age 15 FSIQ scores correlating with midlife IQ $>.75$. As additional data are collected, we will estimate the genetic and environmental factors that mediate these correlations, as well as fit models that explain hypothesized increases in heritability from childhood through middle age.

Grant Support: NIH/NIA grant \#s R01AG063949 and R01 AG063949-02S1.

\section{Resource Profile and User Guide of the Polygenic Index Repository}

Joel Becker ${ }^{1}$, Casper A.P. Burik ${ }^{2}$, Grant Goldman ${ }^{3}$, Nancy Wang ${ }^{3}$, Hariharan Jayashankar ${ }^{3}$, Michael Bennett ${ }^{3}$, Daniel W. Belsky ${ }^{4,5}$, Richard Karlsson Linnér ${ }^{2}$, Rafael Ahlskog ${ }^{6}$, Aaron Kleinman ${ }^{7}$,

David A. Hinds ${ }^{7}$, 23andMe Research Group ${ }^{7}$, Avshalom Caspi ${ }^{8-11}$, David L. Corcoran ${ }^{10}$, Terrie E. Moffitt ${ }^{8-11}$, Richie Poulton ${ }^{12}$,

Karen Sugden ${ }^{8}$, Benjamin S. Williams ${ }^{8}$, Kathleen Mullan Harris ${ }^{13,14}$, Andrew Steptoe ${ }^{15}$, Olesya Ajnakina ${ }^{15,16}$, Lili Milani ${ }^{17}$, Tõnu Esko ${ }^{17,18}$, William G. Iacono ${ }^{19}$, Matt McGue ${ }^{19}$,

Patrik K.E. Magnusson ${ }^{20}$, Travis T. Mallard ${ }^{21}$, K. Paige Harden ${ }^{21,22}$, Elliot M. Tucker-Drob ${ }^{21,22}$, Pamela Herd ${ }^{23}$, Jeremy Freese ${ }^{24}$, Alexander Young ${ }^{25,26}$, Jonathan P. Beauchamp ${ }^{27}$,

Philipp Koellinger ${ }^{2,28}$, Sven Oskarsson ${ }^{6}$, Magnus Johannesson ${ }^{29}$, Peter M. Visscher ${ }^{30}$, Michelle N. Meyer ${ }^{31}$, David Laibson ${ }^{3,32}$,

David Cesarini ${ }^{1,3}$, Daniel J. Benjamin ${ }^{3,25,26}$, Patrick Turley ${ }^{33,34}$, and Aysu Okbay ${ }^{2}$

${ }^{1}$ Department of Economics, New York University, New York, NY, USA

${ }^{2}$ Department of Economics, School of Business and Economics, Vrije Universiteit Amsterdam, Amsterdam, The Netherlands

${ }^{3}$ National Bureau of Economic Research, Cambridge, MA, USA

${ }^{4}$ Department of Epidemiology, Columbia University Mailman School of Public Health, New York, NY, USA

${ }^{5}$ Robert N. Butler Columbia Aging Center, Columbia University, New York, NY, USA

${ }^{6}$ Department of Government, Uppsala University, Uppsala, Sweden
${ }^{7}$ 23andMe, Inc., Mountain View, CA, USA

${ }^{8}$ Department of Psychology and Neuroscience, Duke University, Durham, NC, USA

${ }^{9}$ Social, Genetic, and Developmental Psychiatry Centre, Institute of Psychiatry, Psychology \& Neuroscience, King's College London, London, United Kingdom

${ }^{10}$ Center for Genomic and Computational Biology, Duke University, Durham, NC, USA

${ }^{11}$ Department of Psychiatry and Behavioral Sciences, Duke University, Durham, NC, USA

${ }^{12}$ Dunedin Multidisciplinary Health and Development Research Unit, University of Otago, Dunedin, Otago, New Zealand

${ }^{13}$ Department of Sociology, University of North Carolina at Chapel Hill, Chapel Hill, NC USA

${ }^{14}$ Carolina Population Center, University of North Carolina at Chapel Hill, Chapel Hill, NC USA

${ }^{15}$ Department of Behavioural Science and Health, University College London, London, United Kingdom

${ }^{16}$ Department of Biostatistics and Health Informatics, Institute of Psychiatry, Psychology and Neuroscience, King's College London, London, United Kingdom

${ }^{17}$ Institute of Genomics, University of Tartu, Tartu, Estonia

${ }^{18}$ Broad Institute of MIT and Harvard, Cambridge, MA, USA

${ }^{19}$ Department of Psychology, University of Minnesota, Minneapolis, MN, USA

${ }^{20}$ Swedish Twin Registry, Department of Medical Epidemiology and Biostatistics, Karolinska Institutet, Stockholm, Sweden

${ }^{21}$ Department of Psychology, The University of Texas at Austin, Austin, TX, USA

${ }^{22}$ Population Research Center, The University of Texas at Austin, Austin, TX, USA

${ }^{23}$ McCourt School of Public Policy, Georgetown University, Washington, DC, USA

${ }^{24}$ Department of Sociology, Stanford University, Stanford, CA, USA

${ }^{25}$ UCLA Anderson School of Management, Los Angeles, CA, USA

${ }^{26}$ Human Genetics Department, UCLA David Geffen School of Medicine, Los Angeles, CA, USA

${ }^{27}$ Interdisciplinary Center for Economic Science and Department of Economics, George Mason University, Fairfax, Virginia, USA

${ }^{28}$ Robert M. La Follette School of Public Affairs, University of Wisconsin-Madison, Madison, WI, USA

${ }^{29}$ Department of Economics, Stockholm School of Economics, Stockholm, Sweden

${ }^{30}$ Institute for Molecular Bioscience, The University of Queensland, Brisbane, Queensland, Australia

${ }^{31}$ Center for Translational Bioethics and Health Care Policy, Geisinger Health System, Danville, PA, USA

${ }^{32}$ Department of Economics, Harvard University, Cambridge, MA, USA

${ }^{33}$ Center for Economic and Social Research, University of Southern California, Los Angeles, CA, USA

${ }^{34}$ Department of Economics, University of Southern California, Los Angeles, California, USA

Keywords Polygenic score, Polygenic index, Repository, Measurement error

Polygenic indexes (PGIs) are DNA-based predictors. Their value for research in many scientific disciplines is rapidly growing. As a resource for researchers, we used a consistent methodology to construct PGIs for 47 phenotypes in 11 datasets. To maximize the PGIs' prediction accuracies, we constructed them using genome-wide association studies-some not previously published-from multiple data sources, including 23 andMe and UK Biobank. We present a theoretical framework to help interpret analyses involving PGIs. A key insight is that a PGI can be understood as an unbiased but noisy 
measure of a latent variable we call the "additive SNP factor." Regressions in which the true regressor is the additive SNP factor but the PGI is used as its proxy therefore suffer from errors-in-variables bias. We derive an estimator that corrects for the bias, illustrate the correction, and make a Python tool for implementing it publicly available.

Grant Support: The study was supported by funding from the Ragnar Söderberg Foundation (E42/15, D.C.);, the Swedish Research Council (421-2013-1061, M.J.; 2019-00244, S.O.); an ERC Consolidator Grant (647648 EdGe, P.K.); the Pershing Square Fund of the Foundations of Human Behavior (D.L.);, Open Philanthropy (01062300001, D.J.B., P.T., M.N.M.);, Riksbankens Jubileumsfond P180782:1 (S.O.); Netherlands Organisation for Scientific Research VENI grant 016.Veni.198.058 (A.O.); and the NIA/NIH through grants R24-AG065184 (D.J.B.) and R01-AG042568 (D.J.B.) to the University of California Los Angeles, ; K99-AG062787-01 (P.T.) to Massachusetts General Hospital, and R56-AG058726 (Titus Galama) to the University of Southern California; the NIH/NICHD through grants R01-HD086313 (E.M.T.D.) and R01-HD092548 (K.P.H.) to the University of Texas at Austin; the NIA/NIMH through grants 1R01-MH101244-02 (P.T.; PI: Benjamin M. Neale) and 5U01MH109539-02 (P.T.; PI: B.M.N.) to the Broad Institute at Harvard and MIT; R56-AG058726 (Titus Galama) to the University of Southern California; the Government of Canada through Genome Canada and the Ontario Genomics Institute (OGI-152) (J.P.B.); the Social Sciences and Humanities Research Council of Canada (J.P.B.); the European Union through grant MP1GI18418R (T.E.); the Estonian Research Council through grant PRG1291 (T.E.); the National Health and Medical Research Council through grant GNT113400 (P.M.V.); and the Australian Research Council (P.M.V.).

\section{Genetic Contributions to Memory Ratings and Memory Concerns in Older Men}

Tyler Bell ${ }^{1}$, Nathan Gillespie ${ }^{2}$, Chandra Reynolds ${ }^{3}$, McKenna E. Williams ${ }^{1,4}$, Asad Beck ${ }^{5}$, Carol E. Franz ${ }^{1}$, William S. Kremen ${ }^{1}$

${ }^{1}$ Department of Psychiatry and Center for Behavior Genetics of Aging, University of California San Diego, La Jolla, CA, USA

${ }^{2}$ Virginia Institute for Psychiatric and Behavioral Genetics, Virginia Commonwealth University, Richmond, VA, USA

${ }^{3}$ Department of Psychology, University of California Irvine, Irvine, CA, USA

${ }^{4}$ Department of Psychology, San Diego State University, San Diego, CA, USA

${ }^{5}$ Center for Neurotechnology, University of Washington, Seattle, WA, USA

Keywords Subjective memory, Memory, Twin design, Longitudinal design, Older adults

Subjective memory ratings and memory concerns are associated with increased risk for Alzheimer's disease. Using data from the Vietnam Era Twin Study of Aging, we explored the genetic and environmental etiology of subjective memory ratings and concerns and their covariance with objective memory assessed at ages 56, 62, and 68 years. At each assessment, participants rated their: i) subjective memory ("rate your memory in terms of the kinds of problems you have "and ii) memory concerns ("how concerned you about your memory are?". Responses were dichotomized into reporting any problem in subjective memory, yes or no, or memory concerns, yes or no. Multivariate biometric modeling in OpenMx revealed that subjective memory ratings across time were best explained by a heritable common factor $(\mathrm{A}=0.65,95 \% \mathrm{CI} 0.08-1.22)$. Longitudinal subjective memory concerns were also best explained by a heritable common factor $(\mathrm{A}=.84,95 \% \mathrm{CI} .26,1.42)$. Phenotypic correlations between the objective and each of the subjective memory items were small $\left(\mathrm{r}_{\mathrm{P}}=-.11\right.$ and -.15$)$. The genetic correlation for subjective memory ratings and objective memory was $r_{\mathrm{G}}=-.27$ $(95 \% \mathrm{CI}-.53,-.01)$; for subjective memory concerns and objective memory, $r_{\mathrm{G}}=-.37(95 \% \mathrm{CI}-.63,-.10)$. Our results indicate modest shared genetic influences on the very small shared phenotypic variance between subjective and objective memory. The model-fitting results, showing high heritability of subjective memory ratings and concerns, suggest that they are trait-like, but if they are indicators of imminent dementia-related changes, they should be state-like. Thus, what underlies subjective memory and why it is predictive of dementia remains unclear.

Grant Support: Work on this manuscript was supported through funding from the National Institute on Aging of the National Institutes of Health [grant number NIA R01AG050595, R01 AG022381, P01 AG055367, R01 AG060470.

\section{Sources of Inequality at Birth: The Interplay Between Genes and Parental Socioeconomic Status}

Pietro Biroli ${ }^{1}$, Titus Galama ${ }^{2}$, Andries T. Marees ${ }^{3}$, Pia Arce ${ }^{1}$, Jeremy Vollen ${ }^{1}$

${ }^{1}$ Department of Economics, University of Zurich, Zurich, Switzerland ${ }^{2}$ Center for Economic and Social Research, University of Southern California, Los Angeles, USA

${ }^{3}$ Vrije Universiteit Amsterdam

Keywords Gene-by-Environment Interplay, Polygenic Scores, Social Science Genetics, Inequality

The start of a human's life can be characterized by two lotteries: that of your genes (nature) and that of the family you were born into (nurture). These lotteries set in motion a trajectory, from birth onward, in health and human-capital formation. Genetic factors are implicated in virtually any human trait, and early-life experiences strongly influence long-term outcomes, with children born into high socio-economic status (SES) families generally in better health and of higher SES in later life. Leveraging three longitudinal social-science data sets, the Health and Retirement Study (HRS), the Wisconsin Longitudinal Study (WLS), and the English Longitudinal Study of Aging (ELSA), containing rich genotypic and phenotypic information, we systematically analyze the relationship between an individual's index of genetic propensity towards a trait (genotype), their actual trait in adulthood (phenotype), and the social and economic status of the families they grew up in. We proxy the individual's genetic predisposition to a trait by a polygenic score (PGS) and the SES of the families they were born into by a latent factor of parental SES, constructed from parental education and the father's (former) occupational status. We then investigate how genetic predispositions, socio-economic background, and their interaction contribute to later-life outcomes, across a range of forty-five socioeconomic, anthropometric, health, behavioral, and personality traits. We find strong genetic and socio-economic associations but no evidence for sizeable interactions between them. We discuss several possible implications of this result.

Grant Support: We gratefully acknowledge financial support from NORFACE DIAL (462-16-100), the National Institute on Aging of 
the National Institutes of Health (RF1055654 and R56AG058726), and the Dutch National Science Foundation (016.VIDI.185.044).

\section{Stressful Life Events Increase Risk of Depression: A Population-Based Twin Study}

Ludvig Daae Bjørndal ${ }^{1}$ and Eivind Ystrom $^{2,3,4}$

${ }^{1}$ Department of Psychology, University of Oslo, Oslo, Norway

${ }^{2}$ PROMENTA Research Center, University of Oslo, Oslo, Norway

${ }^{3}$ Department of Mental Disorders, Norwegian Institute of Public Health, Oslo, Norway

${ }^{4}$ School of Pharmacy, University of Oslo, Oslo, Norway

Keywords Stressful life events, Depression, Co-twin control, Twin study, Survival analysis

Previous studies have found that stressful life events (SLEs) are associated with increased risk of adult depression. However, many studies are observational in nature and limited by methodological issues, such as potential confounding by genetic factors. Genetically informative studies, such as the co-twin control design, can strengthen causal inference in observational research. The co-twin control design involves comparing patterns of associations in the full sample and within dizygotic (DZ) and monozygotic twins (MZ). Discrete-time survival analysis has several benefits and multilevel survival analysis can incorporate frailty terms (random effects) to estimate the components of the biometric model. In the current study, we investigated associations between SLEs and depression risk in a population-based twin sample $(\mathrm{N}=2299)$ with a co-twin control design. Associations were modelled using discrete-time survival analysis with biometric frailty terms. SLE occurrence was associated with increased depression risk. Co-twin control analyses indicated that this association was at least in part due to causal influence of SLE exposure on depression risk for event occurrence across all SLEs and of violent SLEs. Stronger within-pair estimates for economic SLEs compared with the full sample association could have resulted if the full sample association was suppressed or if within-pair estimates were inflated. If the former occurred, economic SLEs may represent particularly important risk factors for depression. A minor proportion of the total genetic risk of depression reflected genetic effects related to SLEs. Our findings have implications for future research on SLEs.

Grant Support: This work was supported by a student research grant from the Research Council of Norway.

\section{The Influence of Delinquent Peers on Academic Achievement: Direct Effects or Genetic Predispositions?}

Alicia Bouillon*1, Kathia Couture* ${ }^{1}$, Justine Pleau*1, Sophie Aubé ${ }^{1}$, Catherine Mimeau ${ }^{1}$, Michel Boivin ${ }^{1}$, Mara Brendgen ${ }^{2}$, Frank Vitaro ${ }^{3}$, Richard E. Tremblay ${ }^{3} \&$ Ginette Dionne ${ }^{1}$

*Equal contribution

${ }^{1}$ School of Psychology, Laval University, Quebec City, Quebec, Canada

${ }^{2}$ Department of Psychology, University of Quebec at Montreal, Montreal, Quebec, Canada

${ }^{3}$ Department of Psychology, University of Montreal, Montreal, Quebec, Canada

Keywords Gene-environment correlation, Academic achievement, Delinquent peers, adolescence, Twin study
Affiliation with delinquent peers has been associated with negative outcomes in adolescents' academic achievement ${ }^{1}$. Homophilia, a process by which individuals create friendships with peers who share similar characteristics, could partly explain the affiliation with delinquent peers $^{2}$. Moreover, the association between academic achievement and affiliation with delinquent peers could stem from underlying genetic factors involved in homophilia, rather than from an actual effect of peers. The aims of this study were to determine if the academic achievement of adolescents was associated with their affiliation with delinquent peers, and if this association was explained by genetic factors. The sample was drawn from the Quebec Newborn Twin Study, a longitudinal follow-up of a birth cohort of twins $(n=$ 421 pairs of twins $)^{3}$. Delinquent behaviors and academic achievement were measured when adolescents and their peers were 15 years old. Questionnaires were completed by the twins, their parents, and their peers. Results showed that adolescents' academic achievement was associated with their affiliation with delinquent peers and that this association was completely explained by adolescents' genetic factors. These results suggest that adolescents' academic achievement is explained by their own genetic predispositions rather than influenced by the delinquent behavior of their peers.

References

${ }^{1}$ Gremmen et al. (2019). Child Dev, 90(2), e192-e211.

${ }^{2}$ McPherson et al. (2001). Annu Rev Sociol, 27(1), 415-444.

${ }^{3}$ Boivin et al. (2019). Twin Res Hum Genet, 22(6), 475-481.

Grant Support: Social Sciences and Humanities Research Council of Canada (SSHRC), The Canadian Institute of Health Research (CIHR).

\section{Early Fine Motor Skills and Genetic Associations with Later Educational Achievement and Psychopathology}

\section{Aislinn Bowler ${ }^{1}$, Pasco Fearon ${ }^{2}$, Angelica Ronald ${ }^{1}$}

${ }^{1}$ Centre for Brain and Cognitive Development, Birkbeck, University of London, London WC1E 7HX, United Kingdom

${ }^{2}$ Research Department of Clinical, Educational and Health Psychology, University College London, London, UK

Keywords Motor, Infancy, Polygenic-scores, Neurodevelopmental, Educational-achievement

Fine motor skills are heritable and an important milestone in development. Increasing evidence suggests there are associations between early motor skills and later neurodevelopment and cognition. In a study of 4514 preschool children from the Twins Early Development Study, fine motor assessments were completed by $2-4$-year-olds and their parents as part of a cognitive battery. Tasks included drawing, block building, and folding. A single fine motor factor score was derived from principal component analysis. Polygenic scores (PGS) for traits previously associated with motor skills (ADHD, autism, schizophrenia, years in education) were created along with those where the association is unclear (anxiety, major depressive disorder, obsessive-compulsive disorder). Regression analyses revealed a significant positive association between higher scores in fine motor skills and the higher years in education PGS (GWAS pT, 1, b $=0.24, p<$ $\left..001, \mathrm{R}^{2}=0.004\right)$. A nominal association was found between lower scores in fine motor skills and higher PGS for ADHD (GWAS pT, .01, $\left.\mathrm{b}=-0.15, p=.069, \mathrm{R}^{2}=0.001\right)$. As such, our initial results suggest that fine motor development may be linked to later educational outcomes due to shared common genetic pathways. Following these 
preliminary analyses, the combined effect for all the PGSs will be assessed in a multi-PGS model of fine motor skills.

Grant Support: AB is funded with an MRC Training Grant-UCLBirkbeck DTP (MR/R015759/1). TEDS is supported by a programme grant from the UK Medical Research Council (MR/M021475/1 and previously G0901245).

\section{Genetic and Environmental Influences on the Development of Psychopathology in Individuals With and Without Mild Intellectual Disability}

Bruins, S. ${ }^{1}$, Hartman, C.A. ${ }^{2}$, Lambregts-Rommelse, N.N.J. ${ }^{34}$, Masselink $^{2}$, M.W., Dolan, C.V. ${ }^{1}$, Bergen, E. van ${ }^{1}$, Boomsma, D.I. ${ }^{1}$

${ }^{1}$ Vrije Universiteit, Department of Biological Psychology \& Netherlands Twin Register. Amsterdam, The Netherlands

${ }^{2}$ Faculty of Medical Sciences/UMCG, Interdisciplinary Centre Psychopathology and Emotion regulation (ICPE). Groningen,

The Netherlands

${ }^{3}$ Radboud University Medical Center, Donders Institute for Brain, Cognition and Behavior, Nijmegen

${ }^{4}$ Karakter Child and Adolescent Psychiatry University Center,

Nijmegen, The Netherlands

Keywords Mild Intellectual Disability, Comorbidity, Psychopathology, Genetic Simplex Model, Twin Research

Individuals with mild intellectual disability (MID) are more likely to suffer from psychopathology than their peers without MID, but they are less likely to receive adequate mental health care. Despite the observed comorbidity of MID and psychopathology, little is known about the prevalence, course, and treatment effects in this group (e.g. Dekker et al. 2002). The Netherlands Twin Register (NTR) is part of a consortium with several other large, population-based studies and clinical studies to address these gaps in knowledge.

Here we present our analyses of the development of psychopathology in approximately 500 monozygotic and dizygotic twin pairs concordant or discordant for MID. We use the DSM-based CBCL scales as indicators of psychopathology. We inspect CBCL scores for individuals with MID and without MID, and test for differences between mean CBCL scores of these two groups. We fit an autoregressive genetic simplex model to CBCL scores at ages 3, 7, 9, and 12 and assess (1) the contribution of genes and environment to the variation in psychopathology and the stability of these influences over age; (2) emergence of new genetic or environmental influences during development; and (3) differences in genetic and environmental influences for individuals with and without MID.

Our results will contribute to understanding to what extent and why individuals with MID are at higher risk of psychopathology.

\section{References}

Dekker, M.C., Koot, H.M., Ende, J.V.D., \& Verhulst, F.C. (2002). Emotional and behavioral problems in children and adolescents with and without intellectual disability. Journal of Child Psychology and Psychiatry, 43(8), 1087-1098.

Grant Support: This project is funded by Zonmw grant 636340003 .

\section{Variance Decomposition and Genetic Correlations of Brain Volumes in Children}

Daniel Bustamante ${ }^{1,2}$, Ananda B. Amstadter ${ }^{1,3}$, Michael C. Neale ${ }^{1,3}$

${ }^{1}$ Virginia Institute for Psychiatric and Behavioral Genetics

${ }^{2}$ Integrative Life Sciences Doctoral Program, Virginia Commonwealth University, Richmond, VA, USA

${ }^{3}$ Department of Psychiatry, School of Medicine, Virginia Commonwealth University, Richmond, VA, USA

Keywords Brain, Volume, Genetic, MRI, SEM

Small volumes in certain brain regions of interest (ROIs) have been linked to psychiatric disorders. However, most such work to date have studied adults. Using structural magnetic resonance imaging twin data from the $\mathrm{ABCD}$ study $\left(\mathrm{N}=1,722 ; \mathrm{M}_{\mathrm{age}}=10.12\right)$ and structural equation modeling, we extend this work to children to estimate the genetic and environmental factors influencing the brain volumes of 41 subcortical and 256 cortical ROIs and their genetic correlations. Subcortical ROIs shared-environmental components did not differ significantly from zero and most were below this point. Additive genetic factors explained $43.98-93.60 \%(M=70.21 \%$; $S D=15.90 \%)$ of the variability in the volumes of all subcortical ROIs, except in the inferior lateral ventricles $(\mathrm{LH}=35.71 \%, \mathrm{RH}=32.26 \%)$. Unique environmental factors accounted for $6.40-.56 .01 \%(\mathrm{M}=29.79 \%$; SD $=15.89 \%$ ) of the variance in all subcortical regions aside from the same ROIs $(\mathrm{LH}=64.29 \%, \mathrm{RH}=67.74 \%)$. Subcortical genetic correlations were consistently moderate to high between contiguous (e.g., pallidum-putamen $=.48: .58$ ), and similar structures (e.g., corpus callosum subregions $=.38: .88)$. The caudate nuclei and the lateral ventricles were moderately genetically correlated (.37:.41). These ROIs are contiguous, located medially in the brain, and reveal low to no genetic correlation with the hippocampi $(-.25: .03)$ and the amygdalae ( $-.09: .18)$. Conclusions: Additive genetic factors influenced most of the variability in the volumes of 35 of the 41 subcortical ROIs in children. The absent, low, or negative correlations among medial and distal subcortical ROIs may represent competition between certain adjacent regions, or shared genetic factors influencing their volume in opposite directions. Analyses of cortical ROIs are in progress and we will report the results.

Grant Support: Bustamante: MH020030-21A1 NIMH, Amstadter: K02 AA023239 NIAAA, Neale: U01 DA051037-01 and R01 DA049867-01A1 NIDA.

\section{Understanding the Effects of the COVID-19 Pandemic on Youth Psychopathology: Genotype-Environment Interplay}

Sarah L. Carroll ${ }^{1}$, Elizabeth A. Shewark ${ }^{1}$, Luke W. Hyde ${ }^{2}$, Kelly L. Klump ${ }^{1}$, S. Alexandra Burt ${ }^{1}$

${ }^{1}$ Department of Psychology, Michigan State University, East Lansing, Michigan, USA

${ }^{2}$ Department of Psychology, University of Michigan, Ann Arbor, Michigan, USA

Keywords Coronavirus Pandemic, Youth Psychopathology, Genotype-Environment Interplay

Adversity has consistently been found to predict poor mental health outcomes in children and adolescents. Perhaps the most omnipresent form of adversity in the last several decades is the coronavirus 
pandemic of 2020, a global health crisis that has been linked to elevated rates of numerous forms of youth psychopathology. The ongoing nature of the pandemic renders it critical to identify the mechanisms underlying its effects on mental health. The current study examines pandemic-related disruption across multiple domains (e.g., home life, work, finances) as an etiologic moderator of several common forms of youth psychopathology. Participants were 637 adolescent twin pairs from the Twin Study of Behavioral and Emotional Development in Children (TBED-C). Mothers reported on the disruption experienced by the family using the Epidemic-Pandemic Impacts Inventory. A series of biometric genotype-environment interaction models revealed that disruption augmented the nonshared environmental contributions to emotional distress and conduct problems but had little effect on the etiology of attention-deficit hyperactivity problems. Our results indicate that children in the same family became less alike in their conduct problems and symptoms of emotional distress following exposure to pandemic-related disruption. Furthermore, this process of differentiation occurred regardless of the twins' genetic resemblance. In other words, pandemic-related stress appeared to differentiate twins in their psychopathology, and this was not due to their genetics, or even the experiences they shared, but rather to their own idiosyncratic responses to the current pandemic.

Grant Support: F31HD102094 (SC); F32HD098780 (ES); R01HD066040 (AB); R01-MH081813 (AB).

\section{Gene-Environment Interactions for Childhood Educational Achievement: Do Within-Family Polygenic Score Effects Vary Across Schools and Neighbourhoods?}

Rosa Cheesman, ${ }^{1}$ Nicolai T. Borgen, ${ }^{2}$ Torkild Lyngstad, ${ }^{3}$ Espen M. Eilertsen, ${ }^{1}$ Ziada Ayorech, ${ }^{1}$ Fartein A. Torvik, ${ }^{4,1}$ Ole Andreassen, ${ }^{5}$ Henrik D. Zachrisson, ${ }^{2}$ Eivind Ystrom ${ }^{1,6}$

${ }^{1}$ The PROMENTA Center, Department of Psychology, University of Oslo, Norway

${ }^{2}$ Department of Special Needs Education, Faculty of Educational Sciences, University of Oslo, Norway

${ }^{3}$ Department of Sociology, University of Oslo, Norway

${ }^{4}$ Centre for Fertility and Health, Norwegian Institute of Public Health, Norway

${ }^{5}$ NORMENT, Division of Mental Health and Addiction, Oslo University Hospital \& Institute of Clinical Medicine, University of Oslo, Oslo, Norway

${ }^{6}$ Department of Mental Disorders, Norwegian Institute of Public Health, Oslo, Norway

Keywords Education, Gene-environment interaction, Development, Polygenic scores

Little is known about whether and how children's educational outcomes are shaped by interactions between their genetic propensities and their social environments beyond the family. We link register data on children's standardised test results, schools, and neighbourhoods to the Norwegian Mother, Father and Child Cohort Study (MoBa), which includes $>25,000$ genotyped parent-child trios. We study the effects of children's polygenic scores on their achievements in Mathematics, Reading and English, and estimate gene-environment interactions $(\mathrm{GxE})$ by allowing the effects to vary across schools and neighbourhoods. We then explore contextual differences in how polygenic effects change across ages $10-14$, and use multi-generational data to investigate bias from social sorting (leading to passive gene-environment correlation) and population stratification.
Associations Between Psychiatric Polygenic Risk Scores and General and Specific Psychopathology Symptoms in Childhood and Adolescence: A Co-twin Control Study

Chen, C. ${ }^{1}$, Lu, Y. ${ }^{1}$, Lundström, S. ${ }^{2,3}$, Larsson, H. ${ }^{1,4}$, Lichtenstein, P. ${ }^{1}$, \& Pettersson, E. ${ }^{1}$

${ }^{1}$ Department of Medical Epidemiology and Biostatistics, Karolinska Institutet, Sweden

${ }^{2}$ Centre for Ethics, Law and Mental Health (CELAM), University of Gothenburg, Gothenburg, Sweden

${ }^{3}$ Gillberg Neuropsychiatry Centre, University of Gothenburg, Gothenburg, Sweden

${ }^{4}$ School of Medical Sciences, Örebro University, Örebro, Sweden

Keywords General factor of psychopathology, Polygenic risk scores, Genetic nurture, Multi-polygenic score

Although genetics and psychiatric conditions are correlated, the associations might be indirectly mediated via broad comorbidity, attributable to the correlations among genetics factors, or accounted for by familial factors (including population stratification, dynastic effects, and assortative mating). We estimated direct genetic effects by analyzing associations within dizygotic (DZ) twin pairs, who are perfectly matched for familial factors but vary in genetic similarity due to random allele assignment during meiosis. We regressed a bifactor model based on 98 parent-rated symptoms in childhood $(\mathrm{N}=$ 3,907 DZ twin pairs), and a subsample followed up in adolescence with both self- and parent-ratings on 20 symptoms ( $n=2,393 \mathrm{DZ}$ pairs), onto 10 polygenic risk scores (PRS) between individuals $(\beta)$ and within $\left(\beta_{\mathrm{w}}\right)$ DZ twin pairs. In childhood, the PRS for ADHD predicted general psychopathology $\left(\beta=0.09,[0.06,0.12] ; \beta_{w}=0.07\right.$ $[0.01,0.12])$. Furthermore, the PRS for ADHD predicted specific inattention $\left(\beta=0.04[0.00,0.08] ; \beta_{w}=0.09[0.01,0.17]\right)$ and hyperactivity $\left(\beta=0.07[0.04,0.11] ; \beta_{\mathrm{w}}=0.09[0.01,0.16]\right)$; the PRS for schizophrenia predicted specific learning $(\beta=0.08$ [0.03, 0.13]; $\left.\beta_{\mathrm{w}}=0.19[0.08,0.30]\right)$ and inattention problems $(\beta=0.05[0.01$, $\left.0.09] ; \beta_{\mathrm{w}}=0.10[0.02,0.19]\right)$. In the aggregate, the PRS-general factor associations were similar between individuals and within twin pairs, whereas the PRS-specific factors associations amplified by $84 \%$ within pairs. Genetic factors appear primarily directly associated with psychopathology symptoms, but the pattern might vary for the general and specific factors.

Grant Support: Swedish Research Council (no 2017-01358), Chinese Scholarship Council (CSC201806360008).

\section{Associations Among Adolescent Depressive and Anxiety Symptoms and Early Adult Substance Use: Multivariate Behavioral Genetic Analyses}

Tong Chen ${ }^{1}$, Amanda M. Ramos ${ }^{2}$, Jennifer L. Maggs ${ }^{3}$, David Reiss ${ }^{4}$, Jenae M. Neiderhiser ${ }^{1}$

${ }^{1}$ Department of Psychology, The Pennsylvania State University, University Park, PA, USA

${ }^{2}$ Department of Epidemiology, The University of North Carolina at Chapel Hill, Chapel Hill, NC, USA

${ }^{3}$ Human Development and Family Studies, The Pennsylvania State University, University Park, PA, USA

${ }^{4}$ Yale Child Study Center, Yale School of Medicine, New Haven, CT, USA 


\section{Keywords Depression, Anxiety, Substance use, Longitudinal}

This study examined genetic and environmental influences on associations among depressive and anxiety symptoms in adolescence and substance use in early adulthood. Participants were from the Nonshared Environment and Adolescent Development project, a longitudinal study including same-sex twin/sibling pairs and parents. Adolescent depressive/anxiety symptoms were measured by composite scores of multiple reporters $\left(\mathrm{M}_{\mathrm{age}}\right.$ child $1 / 2=14.4 / 12.9$ years $)$. Substance use was measured by early adult self-reports $\left(\mathrm{M}_{\text {age }}\right.$ child $1 / 2=26.8 / 25.5$ years), including frequency of current use of cigarettes, alcohol, other drugs; problems due to use summed across substances; smoking history (number of years smoked, number of cigarettes ever smoked and smoked per day). Depressive/anxiety symptoms in adolescence were not associated with early adult use or problem use of most substances. Depressive/anxiety symptoms were associated with early adult cigarette use $(r=.12 / .10)$ and smoking history $(r=.20 / .13)$. Multivariate Cholesky biometric models decomposed the shared variance among adolescent depressive symptoms, anxiety symptoms, and early adulthood smoking history into genetic, shared and nonshared environmental influences. Other substance use variables were not included due to small magnitude of associations with depressive/anxiety symptoms. A genetic factor explained variance common to adolescent depressive symptoms, anxiety symptoms, and early adulthood smoking history. No unique association between adolescent anxiety symptoms and early adulthood smoking history was found after accounting for adolescent depressive symptoms. Findings did not suggest adolescent depressive and anxiety symptoms to be differently associated with early adulthood smoking history. Genetic influences were important in explaining the association between internalizing symptoms and smoking behavior.

Grant Support: The Nonshared Environment in Adolescent Development project was supported by National Institute of Mental Health Grant R01MH43373 (T1, David Reiss, PI) and Grant R01MH59014 (T3, Jenae M. Neiderhiser, PI) and by the William T. Grant Foundation (T1, David Reiss, PI). Tong Chen is supported by the Prevention and Methodology Training Program (T32 DA017629, MPIs: Jennifer L. Maggs \& Stephanie T. Lanza) with funding from the National Institute on Drug Abuse. Amanda M. Ramos is supported by National Institute of Environmental Health Sciences F32ES031832.

\section{Exploring the Genetic Overlap of Suicide-Related Behaviors and Substance Use Disorders}

Sarah M.C. Colbert ${ }^{1}$, Alexander S. Hatoum ${ }^{1}$, Andrey Shabalin ${ }^{2}$, Hilary Coon ${ }^{2}$, Elliot C. Nelson ${ }^{1}$, Arpana Agrawal ${ }^{1}$,

Anna R. Docherty ${ }^{2}$, Emma C. Johnson ${ }^{1}$

${ }^{1}$ Department of Psychiatry, Washington University School of Medicine, St. Louis, MO USA

${ }^{2}$ Department of Psychiatry, University of Utah School of Medicine, Salt Lake City, UT USA

Keywords Genome-wide association studies, Substance use disorders, Suicide, Genomic structural equation models, Genetic overlap

Suicide-related behaviors are heterogeneous and transdiagnostic, and may demonstrate varying levels of genetic overlap with different substance use disorders (SUDs). We used linkage disequilibrium score regression and genomic structural equation models to examine the genetic relationships between several SUDs and suicide-related behaviors. Our analyses incorporated summary statistics from the largest genome-wide association studies (GWAS) of problematic alcohol use (PAU), the Fagerström Test for Nicotine Dependence (FTND), cannabis use disorder (CUD), and opioid use disorder (OUD; Ns ranging from 46,213-435,563) and GWAS of ever selfharmed, suicide attempt, and suicide death (Ns ranging from $18,223-117,733)$. We also accounted for genetic liability to depression $(\mathrm{N}=500,199)$ and risk tolerance $(\mathrm{N}=315,894)$. Ever self-harmed correlated most strongly with CUD $\left(r_{\mathrm{g}}=0.60\right)$ and suicide death correlated most strongly with OUD $\left(r_{g}=0.53\right)$. Suicide attempt correlated similarly with all SUDs. Simultaneously correlating a common SUD factor with each specific suicide indicator while controlling for depression and risk tolerance revealed significant, positive genetic correlations between all SUDs and suicide-related behaviors $\left(r_{g}=0.26-0.46\right)$. Our findings suggest that genetic and behavioral contributions to suicide death may somewhat differ from non-lethal suicide-related behaviors. Additionally, we identify a general level of genetic overlap between SUDs and suicide-related behaviors which is independent of depression and risk tolerance.

Grant Support: SMCC, ECJ and AA acknowledge support from MH109532. ECJ acknowledges support through grant YIG-0-064-18 from the American Foundation for Suicide Prevention. The content is solely the responsibility of the authors and does not necessarily represent the official views of the American Foundation for Suicide Prevention. AA acknowledges K02DA032573. ASH receives support from DA007261-17. Support was provided by National Institute of Mental Health R01 MH123619 to AD, R01 MH123489 and R01 MH122412 to HC, a Simons Foundation/SFARI award to AD \& HC, and a Brain \& Behavior Research Foundation Young Investigator award to AS.

\section{Relationship Between Depression and the Impacts of the Responses to the COVID-19 Pandemic in Australia}

Lucía Colodro-Conde $^{1}$, Penelope Lind ${ }^{1}$, Dan Siskind ${ }^{2}$, Ian Hickie ${ }^{3}$, Catherine Olsen ${ }^{4}$, David Whiteman ${ }^{4}$, Nick Martin ${ }^{1}$, Sarah Medland ${ }^{1}$ ${ }^{1}$ Department of Genetics and Computational Biology,

QIMR Berghofer Medical Research Institute, Brisbane, Australia

${ }^{2}$ Queensland Brain Institute, University of Queensland, Brisbane, Australia

${ }^{3}$ Faculty of Medicine and Health, University of Sydney, Sydney, Australia

${ }^{4}$ Department of Population Health, QIMR Berghofer Medical Research Institute, Brisbane, Australia

Keywords COVID-19, Depression, Resilience, Polygenic risk

The COVID-19 pandemic is a strong universal stressor with no recent precedents. To date, Australia has reported few COVID-19 cases and deaths compared to other countries. However, measures to control the pandemic (e.g. social distancing, border closures, lockdowns) are impacting mental health and this impact might be more pronounced in those with a history of or predisposition to mental health disorders. We have collected survey data in two large genotyped cohorts: the QSkin Sun and Health Study (QSkin, a population-based sample of residents in the state of Queensland) and the Australian Genetics of Depression Study (AGDS, a national clinical sample with history of major depressive disorder). The first survey was conducted in mid2020 and focused on the impact of the COVID-19 pandemic on mental health. A follow-up survey will be launched in late April 2021. Preliminary results show that symptoms of depression and anxiety 
significantly worsened after the start of the pandemic. This was true both for individuals with and without a history of depression. We selected the top and bottom decile of polygenic risks scores for depression from a subset of AGDS participants ( $N$ 300 per group). Those with the lowest genetic risk for depression reported being significantly happier and less fidgety/restless in the 3 months prior to the pandemic than those with the highest risk. However, in mid-2020 both groups reported being more sad and fidgety and there were no statistical differences between them. We will expand these results and add new information collected in the 2021 survey.

Grant Support: This project has been funded by Queensland government, Australia (G02138).

\section{Comparison of Algorithm-Based Versus Single-Item Phenotyping Measures of Anxiety and Depression Disorders}

Molly R. Davies ${ }^{\mathrm{a}, \mathrm{b}}$, Joshua E. J. Buckman ${ }^{\mathrm{c}, \mathrm{d}}$, Brett N. Adey ${ }^{\mathrm{a}, \mathrm{b}}$, Chérie Armour ${ }^{\mathrm{e}}$, John R. Bradley ${ }^{\mathrm{f}}$, Susannah C. B. Curzons ${ }^{\mathrm{a}, \mathrm{b}}$, Katrina A. S. Davis ${ }^{\mathrm{a}, \mathrm{b}, \mathrm{g}}$, Kimberley A. Goldsmith ${ }^{\mathrm{a}, \mathrm{b}}$,

Colette R. Hirsch ${ }^{\mathrm{a}, \mathrm{b}, \mathrm{g}}$, Matthew Hotopf ${ }^{\mathrm{a}, \mathrm{b}, \mathrm{g}}$, Christopher Hübel ${ }^{\mathrm{a}, \mathrm{b}, \mathrm{h}, \mathrm{i}}$, Ian R. Jones ${ }^{\mathrm{j}}$, Gursharan Kalsi ${ }^{\mathrm{a}, \mathrm{b}}$, Georgina Krebs ${ }^{\mathrm{a}, \mathrm{g}}$, Yuhao Lin ${ }^{\mathrm{a}, \mathrm{b}}$, Ian Marsh ${ }^{\mathrm{a}, \mathrm{b}}$, Monika McAtarsney-Kovacs ${ }^{\mathrm{a}, \mathrm{b}}$, Andrew M. McIntosh ${ }^{\mathrm{k}}$, Dina Monssen $^{\mathrm{a}, \mathrm{b}}$, Alicia J. Peel ${ }^{\mathrm{a}}$, Henry C. Rogers ${ }^{\mathrm{a}, \mathrm{b}}$,

Megan Skelton ${ }^{\mathrm{a}, \mathrm{b}}$, Daniel J. Smith ${ }^{1}$, Abigail ter Kuile ${ }^{\mathrm{a}, \mathrm{b}}$,

Katherine N. Thompson ${ }^{\mathrm{a}, \mathrm{b}}$, David Veale ${ }^{\mathrm{a}, \mathrm{b}, \mathrm{g}}$, James T. R. Walters ${ }^{\mathrm{d}}$, Roland Zahn ${ }^{\mathrm{a}, \mathrm{b}}$, Gerome Breen ${ }^{\mathrm{a}, \mathrm{b}}$, Thalia C. Eley ${ }^{\mathrm{a}, \mathrm{b}}$

anstitute of Psychiatry, Psychology and Neuroscience, King's College London, Denmark Hill, Camberwell, London, UK

${ }^{\mathrm{b}}$ National Institute for Health Research (NIHR) Biomedical Research Centre, South London and Maudsley Hospital, London, UK

${ }^{\mathrm{c}}$ Centre for Outcomes Research and Effectiveness (CORE), Research Department of Clinical, Educational and Health Psychology, University College London, Gower Street, London WC1E 7HB, United Kingdom

${ }_{\mathrm{d}}$ Cope - Camden and Islington Psychological Therapies Services, Camden \& Islington NHS Foundation Trust, Finsbury Health Centre, Pine Street, London EC1R OLP, United Kingdom

${ }^{\mathrm{e}}$ Stress, Trauma \& Related Conditions (STARC) research lab, School of Psychology, Queens University Belfast (QUB), Belfast, Northern Ireland, UK

${ }^{\mathrm{f}}$ NIHR BioResource, Cambridge University Hospitals, Cambridge Biomedical Campus, Cambridge, UK

${ }^{\mathrm{g}}$ South London and Maudsley NHS Foundation Trust, Denmark Hill, Camberwell, London, UK

${ }^{\mathrm{h}}$ Department of Medical Epidemiology and Biostatistics, Karolinska Institutet, Stockholm, Sweden

${ }^{\mathrm{i}}$ National Centre for Register-based Research, Aarhus Business and Social Sciences, Aarhus University, Aarhus, Denmark

${ }^{\mathrm{j}}$ National Centre for Mental Health, Division of Psychiatry and Clinical Neuroscience, Cardiff University, Cardiff, UK

${ }^{\mathrm{k}}$ Division of Psychiatry, Centre for Clinical Brain Sciences, University of Edinburgh, Edinurgh, UK

${ }^{1}$ Institute of Health and Wellbeing, University of Glasgow, Glasgow, UK

Keywords Anxiety, Depression, Remote assessment, Measurement, Big data

Research to understand the complex etiology of anxiety and depressive disorders often requires large sample sizes, but this comes at a cost. Large-scale studies are typically unable to utilise "gold standard" phenotyping methods, instead relying on remote, self-report measures. Our aim was to assess the comparability of two commonly used phenotyping methods for anxiety and depression disorders. Participants from the GLAD and COPING studies $(\mathrm{N}=$ 58,622 ) received a lifetime algorithm-based diagnosis for five common anxiety disorders and major depressive disorder (MDD). Any anxiety disorder included participants with at least one anxiety disorder. Participants also responded to single-item questions about prior diagnoses by health professionals. Agreement for algorithm-based and single-item diagnoses was high for any anxiety disorder and MDD but low for the individual anxiety disorders. For generalized anxiety disorder (GAD), many participants with a single-item diagnosis did not receive an algorithm-based diagnosis, whereas algorithm-based diagnoses of the other anxiety disorders were more common than the single-item diagnoses. The phenotyping methods were moderately comparable for any anxiety disorder and MDD cases. Frequencies of specific anxiety disorders varied depending on the method. Future investigations of specific anxiety disorders should use algorithm-based or other robust phenotyping methods.

Grant Support: This work was supported by the National Institute of Health Research (NIHR) BioResource [RG94028, RG85445], NIHR Biomedical Research Centre [IS-BRC-1215-20018], HSC R\&D Division, Public Health Agency [COM/5516/18], MRC Mental Health Data Pathfinder Award (MC_PC_17,217), and the National Centre for Mental Health funding through Health and Care Research Wales.

\section{The Role of Serotonergic and Inflammatory System Genes in Individual Differences in Depression Level}

Yuliya Davydova ${ }^{1}$, Anastasiya Kazantseva ${ }^{1}$, Renata Enikeeva ${ }^{1}$, Rustam Mustafin ${ }^{2}$, Zalina Takhirova ${ }^{3}$, Marina Lobaskova ${ }^{3}$, Sergey Malykh ${ }^{3,4}$, Elza Khusnutdinova ${ }^{1,4}$

${ }^{1}$ Institute of Biochemistry and Genetics - Subdivision of the Ufa Federal Research Centre of the Russian Academy of Sciences, Ufa, Russian Federation

${ }^{2}$ Department of Medical Genetics and Fundamental Medicine, Bashkir State Medical University, Ufa, Russian Federation

${ }^{3}$ Psychological Institute of the Russian Academy of Education, Moscow, Russian Federation

${ }^{4}$ Lomonosov Moscow State University, Moscow, Russian Federation

Keywords Depression, Serotonin, Inflammation, GxE-interaction

Depression is an important public health issue due to its high prevalence and socio-economic costs to society. A multifactorial nature of depression suggests the involvement of multiple genes of small effect and environmental factors in its development, thus requiring simultaneous examination of genetic and environmental predictors. Considering the role of serotonergic and inflammatory functioning in emotional regulation, the present study aimed to estimate both the main effect of polymorphic variants of serotonergic (SLC6A4 5-HTTLPR, HTR1B rs13212041, HTR2A rs7322347) and inflammatory (TNF rs1041981, CRP rs3093077, IL1B rs16944) system genes, and GxE-interactions in individual differences in depression in healthy individuals. The study included 999 mentally healthy individuals ( $80.5 \%$ women; $19.98 \pm 1.80$ years) of Caucasian origin (327 Russians, 242 Tatars, 233 Udmurts, 112 Bashkirs and 85 of mixed ethnicity) from Russia. Depression was assessed using BDI. SNPs genotyping was performed used Real-Time PCR. Statistical analysis was conducted with PLINK v.1.9 followed by FDR correction for multiple testing. Statistical analysis revealed association of 
the TNF rs1041981 A-allele and decreased depression level in Russians $(\beta=-1.44 ; \mathrm{P}=0.04)$, while the SLC6A4 5-HTTLPR LLgenotype was statistically significantly more frequent in men with enhanced depression score $\left(\beta=3.79 ; \mathrm{P}=0.009 ; \mathrm{P}_{\mathrm{FDR}}=0.13\right)$. As a result of stratification analysis we observed that birth season significantly affected association of TNF rs1041981 $(\beta=-2.21 ; \mathrm{P}=0.006$; $\left.\mathrm{P}_{\mathrm{FDR}}=0.04\right), I L 1 B$ rs $16944(\beta=-2.21 ; \mathrm{P}=0.04)$ and depression level in the total sample. The present study provides evidence that $T N F, I L 1 B$ and $S L C 6 A 4$ genetic variants may contribute to depression susceptibility together with environmental factors in mentally healthy young adults.

\section{Comparing Designs for Estimating Parental Indirect Genetic Effects}

Perline A Demange ${ }^{1}$, Michel Nivard ${ }^{1}$, Espen M Eilertsen ${ }^{2}$, Rosa Cheesman $^{2}$

${ }^{1}$ Vrije Universiteit Amsterdam

${ }^{2}$ PROMENTA Research Center, Department of Psychology, University of Oslo

Keywords Indirect genetic effects, Genetic nurture, Adoption, Siblings, Non transmitted alleles

There has been a recent interest in separating direct genetic effects (influence of the individual's genome on their own outcomes) from parental indirect genetic effects (influence of parental genetics on individuals' outcomes). Three key designs have been developed to estimate direct and indirect genetic effects using polygenic scores, which respectively use adoption, sibling, and parent-offspring trio data. To complement our empirical use of three designs to estimate indirect genetic effects of non-cognitive skills (Demange et al. 2020), we use data simulation to compare these designs in presence of different biases and indirect effect components. We simulate data with or without presence of parental indirect effects, indirect effects of siblings, pre- and postnatal indirect effects, and assortative mating. We show that designs are not equal in their estimation of indirect genetic effects. Adoption data are necessary to separate pre- and postnatal indirect effects. Assortative mating biases sibling and triobased estimates with or without a parental indirect genetic effect, but only affects the adoption design in the presence of parental indirect effects. Designs using siblings are very sensitive to the choice of estimates to represent the population genetic effect, and to the presence of sibling effects. Our results call for a specific attention to the design used when interpreting estimates of indirect genetic effects.

\section{References}

Demange, P.A., et al. (2020). Parental influences on offspring education: indirect genetic effects of non-cognitive skills. BioRxiv. 1-39. https://doi.org/10.1101/2020.09.15.296236

Grant Support: PAD is supported by the grant 531003014 from The Netherlands Organization for Health Research and Development (ZonMW). MGN is supported by R01MH120219, ZonMW grants 849200011 and 531003014 from The Netherlands Organization for Health Research and Development, a VENI grant awarded by NWO (VI.Veni.191G.030) and is a Jacobs Foundation Research Fellow.

\section{Teaching Human Behavior Genetics}

Jaime Derringer ${ }^{1}$

${ }^{1}$ Department of Psychology, University of Illinois at Urbana-Champaign, Champaign, Illinois, USA

\section{Keywords Education, Methods, Pedagogy}

Students in behavior genetics classes are diverse in their educational and cultural backgrounds and goals. One thing they share is living at a time of rapidly developing genetic technology often marketed for personal decision making. In our research products, we call for consideration of impacts and regulation of applications and urge caution above all else. Nonetheless, history shows that new technologies, especially those applied to reproductive or medical decision-making, become more widely accepted over time. Regulation of genetic technologies has been slow and more often than not fails to reach a clear consensus. Although classroom-based education is currently one of our most common forms of public outreach, there has been little formal discussion of pedagogical practices in human behavior genetics. The social relevance of our science demands that we consider the 'Who', 'What', and 'Why' of our teaching practices. I will present a review of structures and topics typical in human behavior genetics courses and explore how these approaches do or do not meet goals for broadly targeted, introductory-level instruction within our field. I will also discuss the shifting goals for behavior genetic education, including training students as both producers and consumers of research. A modular course design enables ongoing updates, with the ability to rotate topics, sources, and approaches as needed to meet a variety of objectives. Educational models to develop critical thinking skills allow students to engage with emerging behavior genetic research well beyond the bounds of a traditional college course.

\section{Distinguishing Well-Being and Resilience: A GWAS-by-Subtraction Study in UK Biobank}

Lianne P. de Vries ${ }^{1,2}$, Perline A. Demange ${ }^{1,2}$, Dirk H.M. Pelt ${ }^{1,2}$, Christiaan H. Vinkers ${ }^{3,4}$, Michel G. Nivard ${ }^{1,2}$, \& Meike Bartels ${ }^{1,2}$

${ }^{1}$ Department of Biological Psychology, Vrije Universiteit Amsterdam, The Netherlands

${ }^{2}$ Amsterdam Public Health Research Institute, Amsterdam University Medical Centres, Amsterdam, The Netherlands

${ }^{3}$ Department of Psychiatry, Amsterdam UMC, Location VUmc, The Netherlands

${ }^{4}$ Department of Anatomy and Neurosciences, Amsterdam UMC, Location VUmc, The Netherlands

Keywords Well-being, Resilience, GWAS-by-subtraction

Resilience can be defined as the ability to quickly recover after experiencing stress, returning to an optimal mental state. In a previous study, we reported a strong phenotypic relation $(r=.50)$, a large overlap and possible bidirectional causality between resilience and well-being (de Vries et al. 2021). In a bivariate twin model, the genetic and environmental correlations between resilience and wellbeing were respectively .71 (95\% CI $0.70-0.71)$, and .93 (95\% CI 0.86-0.98), indicating a large overlap in the genetic and environmental factors underlying resilience and well-being. The strong phenotypic, genetic, and environmental correlations between wellbeing and resilience suggests a partly overlapping etiology, which sometimes leads to the claim that well-being is resilience and that the same trait is assessed but with a different name. In an ongoing project, 
we further investigate the relation, overlap and differences between resilience and well-being using molecular genetic analyses. First, we perform a GWAS on UK Biobank data to investigate the genetic variants related to resilience. Next, we apply the GWAS-by-subtraction method (Demange et al. 2021) using the UKB happiness GWAS of Baselmans and Bartels (2018) and our UKB resilience GWAS to directly investigate the overlap between happiness and resilience. Subtracting the resilience GWAS from the happiness will lead to a GWAS of "pure happiness", by removing genetic variants associated with resilience. In follow-up analyses, we investigate and compare the genetic variants associated with resilience, happiness, and "pure happiness" using functional annotation, gene-mapping, and gene-based tests and we compute genetic correlations with a range of phenotypes.

\section{References}

Baselmans BML, Bartels M (2018) A genetic perspective on the relationship between eudaimonic - and hedonic well-being. Sci Rep 8:. https://doi.org/10.1038/s41598-018-32638-1

de Vries LP, Baselmans BML, Luykx JJ, et al (2021) Genetic evidence for a large overlap and potential bidirectional causal effects between resilience and well-being. Neurobiol Stress 14:100315. https://doi.org/10.1016/j.ynstr.2021.100315

Demange PA, Malanchini M, Mallard TT, et al (2021) Investigating the genetic architecture of noncognitive skills using GWAS-by-subtraction. Nat Genet. https://doi.org/10.1038/s41588-020-00754-2

Grant Support: This work is supported by an ERC consolidator grant (WELL-BEING 771057, M. Bartels).

\section{Multivariate Analysis Reveals Shared Genetic Architecture of Brain Morphology and Human Behavior}

R. de Vlaming ${ }^{1}$, Eric A.W. Slob ${ }^{2,3,4}$, Philip R. Jansen ${ }^{5,6}$, Alain Dagher ${ }^{7}$, Philipp D. Koellinger ${ }^{1,8}$, Patrick J.F. Groenen ${ }^{9}$, Cornelius A. Rietveld ${ }^{2,3}$

${ }^{1}$ School of Business and Economics, Vrije Universiteit Amsterdam, Amsterdam, The Netherlands

${ }^{2}$ Department of Applied Economics, Erasmus School of Economics, Rotterdam, The Netherlands

${ }^{3}$ Erasmus University Rotterdam Institute for Behavior and Biology, Erasmus School of Economics, Rotterdam, The Netherlands

${ }^{4}$ MRC Biostatistics Unit, School of Clinical Medicine, University of Cambridge, Cambridge, UK

${ }^{5}$ Department of Complex Trait Genetics, Center for Neurogenomics and Cognitive Research, Amsterdam Neuroscience, Vrije Universiteit Amsterdam, Amsterdam, The Netherlands

${ }^{6}$ Department of Clinical Genetics, VU Medical Center, Amsterdam UMC, Amsterdam, The Netherlands

${ }^{7}$ Montreal Neurological Institute, McGill University, Montreal, Quebec, Canada

${ }^{8}$ La Follette School of Public Affairs, University of WisconsinMadison, WI, USA

${ }^{9}$ Econometric Institute, Erasmus School of Economics, Rotterdam, The Netherlands

Keywords Multivariate GREML, Genetic correlation, Brain morphology, Human behavior

Human variation in brain morphology and behavior are related and highly heritable. Yet, it is largely unknown to what extent specific features of brain morphology and behavior are genetically related.
Here, we introduce multivariate genomic-relatedness restricted maximum likelihood (MGREML) and provide estimates of the heritability of grey-matter volume in 74 regions of interest (ROIs) in the brain. We map genetic correlations between these ROIs and health-relevant behavioral outcomes including intelligence. We find four genetically distinct clusters in the brain that are aligned with standard anatomical subdivision in neuroscience. Behavioral traits have distinct genetic correlations with brain morphology which suggests trait-specific relevance of ROIs.

Grant Support: UK Biobank has obtained ethical approval from the National Research Ethics Committee (11/NW/0382). This research has been conducted using the UK Biobank Resource under application number 11425. We would like to thank the participants and researchers from UK Biobank Imaging Study who significantly contributed or collected data. This work was carried out on the Dutch national e-infrastructure with the support of SURF Cooperative (NWO Call for Compute Time EINF-403 to E.A.W.S.). P.D.K. and R.d.V. were supported by a European Research Council Consolidator Grant (647648 EdGe to P.D.K.). P.D.K. was also supported by the Office of the Vice Chancellor for Research and Graduate Education at the University of Wisconsin-Madison with funding from the Wisconsin Alumni Research Foundation. C.A.R. was supported by a European Research Council Starting Grant (946647 GEPSI).

\section{The Genetics of Assortative Mating}

\author{
Melanie MP de Wit $^{1}$, Karin JH Verweij ${ }^{2}$, Abdel Abdellaoui ${ }^{2}$, \\ ${ }^{1}$ Department of Biological Psychology, \\ Vrije Universiteit van Amsterdam, Amsterdam, The Netherlands \\ ${ }^{2}$ Department of Psychiatry, Amsterdam Universitair Medisch Cen- \\ trum (UMC), Amsterdam, The Netherlands
}

Keywords Assortative mating, Genetics, Behaviour, Complex traits

People tend to choose a partner that is alike; a phenomenon called (positive) assortative mating. This behaviour is apparent in a wide variety of characteristics, including physical characteristics, personality, educational level and socioeconomic status. Such behaviour can have substantial impact on the genetic make-up of subsequent generations. Yet, research has only addressed the underlying genetics of assortative mating in a sparse selection of traits. Therefore, we investigate genetic assortative mating in a broad range of traits regarding health, personality, cognition, behaviour and physical dimensions. In a sample of over 46.000 spouse pairs in the UK Biobank, we investigate the spousal resemblance based on polygenic scores for a range of traits. Furthermore, we compute genome-wide per-SNP spouse correlations and apply LD score regression (BulikSullivan et al., 2015) to assess the genetic correlations of spousal resemblance with complex traits. Based on previous research we expect to find significant assortative mating for traits related to behaviour, health, and socioeconomic status. This work can offer novel insights on the relationship between mate choice and genetic variation related to complex traits.

\section{References}

Bulik-Sullivan, B., Finucane, H. K., Anttila, V., Gusev, A., Day, F. R., Loh, P. R., ... Genetic Consortium for Anorexia Nervosa of the Wellcome Trust Case Control Consortium 3. (2015). An atlas of genetic correlations across human diseases and traits. Nature Genetics, 47(11), 1236-41. https://doi.org/10.1038/ng.3406

Howe, L. J., Lawson, D. J., Davies, N. M., St. Pourcain, B., Lewis, S. J., Davey 


\section{Sex Differences in the Biometrical Model for Substance Use Vulnerability}

\author{
Deepika R. Dokuru ${ }^{1}$, Maia Frieser ${ }^{1}$, John K. Hewitt ${ }^{1}$, \\ Michael C Stallings ${ }^{1}$ \\ ${ }^{1}$ Department of Psychology and Neuroscience, and Institute for \\ Behavioral Genetics, University of Colorado Boulder, USA
}

Keywords Substance use, Comorbidity, Genetic etiology, Twins

To date, numerous studies that have studied sex differences in substance use have found significant differences in the etiology of use trajectories in males and females. In this study, we investigated sex differences in the change of variance components over time for a general factor of polysubstance use dependence. We used data from the Colorado longitudinal twin studies $(\mathrm{N}=2884)$. Analyses utilized transformed (ordinal) DSM dependence counts based on the number of DSM IV dependence criteria endorsed for each substance. Although DSM-IV dependence criteria scores range from 1 to 7 , due to substantial skew and sparsity of extreme scores in a community twin sample, we binned the symptom counts into 0- "No symptoms" and 1- "Some Symptoms." In addition, all substances outside of tobacco, alcohol, and cannabis were collapsed into one variable described as "other" and the substance with the highest dependence criteria endorsement was used. Across assessment waves (adolescence to adulthood) in females, the influence of the genetic variance component increased, and the influence of the shared environment component decreased. In males, we see the opposite trend, where the genetic variance component declines with age and effect of the shared environment peaks in early adulthood before declining slightly. In both males and females, the effects of nonshared environment increases gradually with age. This inconsistent contribution of genetic and environmental influence between males and females is indicative that sex differences in drug use are not wholly biological or sociocultural and their interactive effects lead to substantial phenotypic differences observed.

Grant Support: P50 DA011015, T32DA017637.

\section{Self-perception of Risk for Developing Substance Use Disorders}

Morgan N. Driver ${ }^{1}$, Sally I-Chun Kuo ${ }^{2}$, Peter B. Barr ${ }^{2}$, Fazil Aliev², Spit for Science Working Group, \& Danielle M. Dick ${ }^{1,2}$

${ }^{1}$ Department of Human and Molecular Genetics, Virginia Commonwealth University, Richmond, Virginia, USA

${ }^{2}$ Department of Psychology, Virginia Commonwealth University, Richmond, Virginia, USA

\section{Keywords Risk perception, Substance use disorders}

Risk perception, the perceived likelihood of experiencing a healthrelated outcome, is associated with a number of preventative and health-related behavioral outcomes including health care service use, uptake of screening, diet, and exercise. Perceived risk has been studied in relation to health outcomes such as cancer and diabetes, but little research has examined perceived risk as it relates to substance use disorders, including alcohol use disorder, nicotine use disorder, and cannabis use disorder, which are influenced by both genetic factors and environmental factors. We conducted a brief survey with participants recruited from a registry which enrolled cohorts of freshmen at an urban public university; 207 participants $(76.2 \%$ female; $58.3 \%$ self-reported as White; Mean age $=24.48$ years) completed the survey assessing self-perception of risk for developing substance use disorders (seven-point response scale from "no chance" to "certain to happen") and factors that contribute to their risk perception. The percentage of participants who believed that they had at least a "moderately likely" chance of developing alcohol use disorder, nicotine use disorder, and cannabis use disorder was $34.3 \%$, $20.3 \%$ and $23.9 \%$, respectively. Additionally, $63.3 \%$ of the sample reported that their risk for developing alcohol use disorder was based on family history, while only $18.8 \%$ based their risk for cannabis use disorder on family history. We also examine the relationship between self-reported risk factors and risk perception, as well as substance use. These results can be used to inform prevention efforts, including educational resources surrounding the complex etiology of substance use disorders.

Grant Support: K02AA018755 and R34AA027347.

\section{The Effect of AVPR1A, GATA2, and PCDH7 Gene Variants on Developing Music Abilities in Russian Students}

Renata Enikeeva $^{1,2}$, Anastasiya Kazantseva ${ }^{1,2}$, Yuliya Galyautdinova ${ }^{3}$, Yuliya Davydova ${ }^{1}$, Marina Lobaskova ${ }^{4}$, Elza Khusnutdinova ${ }^{1,5}$, Sergey Malykh ${ }^{3,5}$

${ }^{1}$ Department of Human Molecular Genetics, Institute of Biochemistry and Genetics - Subdivision of the Ufa Federal Research Centre of the Russian Academy of Sciences, Ufa, Russian Federation

${ }^{2}$ Centre for interdisciplinary research in the field of educational sciences, Russian Academy of Education, Moscow, Russian Federation ${ }^{3}$ Department of Genetics and Fundamental Medicine, Bashkir State University, Ufa, Russian Federation

${ }^{4}$ Laboratory of age psychogenetics, Psychological Institute of the Russian Academy of Education, Moscow, Russian Federation

${ }^{5}$ Faculty of psychology, Lomonosov Moscow State University, Moscow, Russian Federation

Keywords Depression, Oxytocin, Gene $\times$ Environment interactions, Birth order, Haplotype

According to neurobiological hypothesis, the development of music abilities is a complex process affected by multiple genes, epigenetic and environmental components. Twin research demonstrated that the coefficient of inheritance of music pitch and rhythm accounted for $70-80 \%$. It was suggested that molecular pathways regulating prosocial behavior and music perception might be responsible for individual differences in music abilities. Namely, association studies linked genetic variants in the arginine vasopressin receptor gene (AVPR1A), GATA transcription factor (GATA2) and protocadherin 7 $(P C D H 7)$ to music perception and memory. The present study aimed to estimate the main effect of AVPR1A RS1, GATA2 rs9854612 and $P C D H 7$ rs13146789 gene variants in developing music abilities in Russian students. The study included musically talented students, who passed through the severe contest to study in musical conservatories ( $\mathrm{N}=100,63 \%$ women; aged 18-22 years) in Moscow (Russia). Control group consisted of mentally healthy individuals $(\mathrm{N}=100)$ corresponding by age, sex, and ethnicity to the group of musically talented individuals. SNPs genotyping was performed using PCRbased KASP genotyping technology on "CFX96" DNA Analyzer. Statistical testing of logistic regression models was performed with PLINK v.1.09. We succeeded to observe an increased frequency of PCDH7 rs 13146789 T-allele $\left(\chi^{2}=4.51, \mathrm{P}=0.033\right.$, OR $1.60,95 \% \mathrm{CI}$ $1.03-2.47)$ and TT-genotype $\left(\chi^{2}=4.28, \mathrm{P}=0.039\right.$, OR $2.8,95 \% \mathrm{CI}$ 1.31-6.96) in musically talented students. However, other examined 
gene variants failed to be associated with music aptitude. The impact of $\mathrm{PCDH} 7$ variants in musical abilities are congruent to previously reported protocadherins involvement in amygdale developmentbrain region related to emotion recognition for music.

Grant Support: This study was supported by the Russian Science Foundation (project no. 17-78-30028).

\section{Insulinopathies of the Brain? Genetic Overlap Between Somatic Insulin-Related and Neuropsychiatric Disorders}

Giuseppe Fanelli ${ }^{1,2}$, Barbara Franke ${ }^{2,3}$, Ward De Witte ${ }^{2}$, I. Hyun Ruisch ${ }^{4}$, Jan Haavik ${ }^{5}$, Veerle van Gils ${ }^{6}$, Willemijn J. Jansen ${ }^{6}$, Stephanie J. B. Vos ${ }^{6}$, Lars Lind ${ }^{7}$, Jan K. Buitelaar ${ }^{8}$, Tobias Banaschewski ${ }^{9}$, Søren Dalsgaard ${ }^{10,11}$, Alessandro Serretti ${ }^{1}$, Nina Roth Mota ${ }^{2}$, Geert Poelmans ${ }^{2}$, Janita Bralten $^{2}$

${ }^{1}$ Department of Biomedical and Neuromotor Sciences, University of Bologna, Bologna, Italy

${ }^{2}$ Department of Human Genetics, Radboud University Medical Center, Donders Institute for Brain, Cognition and Behaviour, Nijmegen, The Netherlands

${ }^{3}$ Department of Psychiatry, Radboud University Medical Center, Donders Institute for Brain, Cognition and Behaviour, Nijmegen, The Netherlands

${ }^{4}$ Department of Child and Adolescent Psychiatry, University of Groningen, University Medical Center Groningen, Groningen, The Netherlands

${ }^{5}$ Department of Biomedicine, University of Bergen, Bergen, Norway ${ }^{6}$ Department of Psychiatry and Neuropsychology, School for Mental Health and Neuroscience, Maastricht University, Maastricht, The Netherlands

${ }^{7}$ Department of Medical Sciences, Uppsala University, Uppsala, Sweden

${ }^{8}$ Department of Cognitive Neuroscience, Donders Institute for Brain, Cognition and Behaviour, Radboud University Medical Center, Nijmegen, The Netherlands

${ }^{9}$ Department of Child and Adolescent Psychiatry and Psychotherapy, Central Institute of Mental Health, Medical Faculty Mannheim, Heidelberg University, Mannheim, Germany

${ }^{10}$ National Centre for Register-Based Research, Aarhus University, Aarhus, Denmark

${ }^{11}$ The Lundbeck Foundation Initiative for Integrative Psychiatric Research, PSYCH, Aarhus, Denmark

Keywords Insulin, Pleiotropy, Alzheimer's disease, Obsessive-compulsive disorder, Autism spectrum disorder

The prevalence of Alzheimer's disease (AD), autism spectrum disorder (ASD), and obsessive-compulsive disorder (OCD) is higher among patients with somatic insulinopathies, like metabolic syndrome (MetS), obesity, and type 2 diabetes mellitus (T2DM). Dysregulation of insulin signalling has been implicated in these neuropsychiatric disorders, and shared genetic factors might partly underlie these observed comorbidities. We investigated genetic overlap between AD, ASD, and OCD with MetS, obesity, and T2DM by estimating pairwise genetic correlations using the summary statistics of the largest available genome-wide association studies for these diseases. Stratified covariance analyses were performed to investigate the contribution of insulin-related gene-sets. Having tested these hypotheses, novel brain "insulinopathies" were explored by estimating the genetic relationship of six additional neuropsychiatric disorders with nine insulin-related diseases/traits. Significant genetic correlations were found between ASD and MetS $\left(\mathrm{r}_{\mathrm{g}}=0.115, \mathrm{p}=\right.$ $0.002)$, OCD and MetS $\left(r_{g}=-0.315, p=3.9 e-8\right)$, OCD and obesity $\left(\mathrm{r}_{\mathrm{g}}=-0.379, \mathrm{p}=3.4 \mathrm{e}-5\right)$, and OCD and T2DM $\left(\mathrm{r}_{\mathrm{g}}=-0.172, \mathrm{p}=\right.$ $3 \mathrm{e}-4)$. Stratified analyses showed negative genetic covariances between ASD and MetS/T2DM through gene-sets comprising insulin signalling and/or insulin processing genes, and between AD/OCD and MetS/T2DM through an insulin receptor recycling gene-set $(p<$ $6.17 \mathrm{e}-4)$. Significant genetic correlations with insulin-related phenotypes were also found for anorexia nervosa, attention-deficit/ hyperactivity disorder, major depression, and schizophrenia $(\mathrm{p}<$ $6.17 \mathrm{e}-4)$. Our findings highlight genetic overlap of somatic insulinrelated phenotypes with multiple neuropsychiatric disorders, pointing to a shared etiology. These results represent a starting point for a new research line on "insulinopathies" of the brain, which may support the development of more effective/tolerated treatment strategies for neuropsychiatric disorders.

Grant Support: This project has received funding from the European Union's Horizon 2020 research and innovation programme under grant agreement No 847879 (PRIME, Prevention and Remediation of Insulin Multimorbidity in Europe).

\section{SES Differences in Longitudinal Dynamic Relationship Between Temperament and Cognition in Childhood: The Louisville Twin Study}

Deborah Finkel $^{1,2}$, Deborah W. Davis ${ }^{3}$, Evan J. Giangrande ${ }^{4}$, Sean Womack ${ }^{4}$, Eric Turkheimer ${ }^{4}$, and Christopher Beam ${ }^{5}$

${ }^{1}$ Department of Psychology, Indiana University Southeast, New Albany, Indiana, USA

${ }^{2}$ Institute for Gerontology, Jönköping University, Jönköping, Sweden ${ }^{3}$ University of Louisville, Louisville, Kentucky, USA

${ }^{4}$ University of Virginia, Charlottesville, Virginia, USA

${ }^{5}$ University of Southern California, Los Angeles, California, USA

Keywords Cognition, Temperament, Childhood, Longitudinal, SES

Neurocognitive theories of early childhood development recognize the dynamic and interactive relationships between cognitive and emotional development as described by the construct of temperament. Although once thought to be relative stable over time, it is now known that the self-regulation of emotional reactivity emerges in late infancy, continues to develop throughout childhood, and is associated with the control over attentional processes. At the same time, cognitive control is simultaneously developing. The trajectories of these two related and interactive systems have not been explored longitudinally in a genetically informed sample. Longitudinal assessments of mental development (MD) and temperament were collected from 486 twins from ages 4 to 9 years as part of the Louisville Twin Study (70\% with 3 or more waves). Factor analysis of 8 temperament scales from the McDevitt Style Questionnaire and the Temperament in Middle Childhood Questionnaire resulted in 3 factors consistent across waves: reactivity (intensity, distress, and threshold), persistence (persistence and activity), and approach (approach, adaptability, and mood). Mental development was assessed with the age-appropriate scale (Stanford-Binet, WPPSI, or WISC). Model-fitting indicated significant SES differences in the temporal dynamics between mental development and temperament: the strongest relationships identified were between temperament and subsequent mental development in higher SES children. Twin analysis indicated that temporal coupling impact primarily genetic variance indicating that for the higher SES group a significant portion of the genetic 
variance in mental development arises from genetic variance for temperament. Thus, results indicate that the relationship between mental development and temperament is moderated by SES.

Grant Support: National Institute of Aging 1R03AG048850-01 \& R01AG063949-01.

\section{Socioeconomic Status Moderates Heritability of Entrepreneurial Personality Factor}

\author{
Deborah Finkel ${ }^{1,2}$, Karin Hellerstedt ${ }^{3}$, Lucia Naldi ${ }^{3}$ \\ ${ }^{1}$ Department of Psychology, Indiana University Southeast, New \\ Albany, Indiana, USA \\ ${ }^{2}$ Institute for Gerontology, Jönköping University, Jönköping, Sweden \\ ${ }^{3}$ Jönköping International Business School, Jönköping University, \\ Jönköping, Sweden
}

The entrepreneurship literature has generally assumed that the tendency to engage in entrepreneurial activity was driven by learning or situational factors. Recent twin and genetic studies have identified significant genetic influences on measures of entrepreneurship. However, to date few studies have examined the extent to which environmental factors might moderate genetic influences on entrepreneurship. Full occupational history was available for 569 individuals from the Swedish Adoption Twin Study of Aging, allowing for identification of entrepreneurs. Personality variables that differentiated entrepreneurs from non-entrepreneurs were identified (activity, extraversion, hard-driving, life direction, monotony avoidance, and sociability) and an entrepreneurial personality factor (EPF) was created. Four measures of environmental support (education, childhood SES, childhood urban/rural, adulthood urban/rural) were intercorrelated, but both education and childhood urban/rural contributed independently to the prediction of EPF. Moderation of genetic influences on EPF by environmental factors was examined in a sample of $210 \mathrm{MZ}$ pairs and $366 \mathrm{DZ}$ pairs aged 26-92. For all 4 environmental support variables, genetic variance was significantly lower (approximately $20 \%$ vs. $60 \%$ ) in environments that provided less support for entrepreneurship: rural childhood, rural adulthood, lower education, and lower SES. The trend for lower unique environmental variance in environments supportive of entrepreneurship was significant for education and childhood SES. Thus, results provide support for a version of the diathesis-stress model of gene-environment interplay: both a genetic predisposition for entrepreneurship and an environment conducive to entrepreneurial activity are required to develop the phenotype.

\section{A Behavioral Genetic Analysis of Relationships Between Executive Functions, Intelligence, Impulsivity, and Multiple Reports of Adolescent Behavioral Problems}

\author{
Samantha M. Freis ${ }^{1,2}$, Jordan D. Alexander ${ }^{3}$, Robin Corley ${ }^{1,2}$, \\ Scott I. Vrieze ${ }^{1,3}$, Naomi P. Friedman ${ }^{1,2}$ \\ ${ }^{1}$ Institute for Behavioral Genetics, University of Colorado Boulder \\ ${ }^{2}$ Department of Psychology and Neuroscience, University of Color- \\ ado Boulder \\ ${ }^{3}$ Department of Psychology, University of Minnesota
}

Keywords Self-regulation, Executive control, Cognitive ability, Psychopathology, Heritability

Executive functioning (EF) and impulsivity measures both relate to psychopathology. However, self-report measures of impulsivity and laboratory EF tasks typically display small correlations, raising the question of whether impulsivity and EFs tap separate aspects of selfregulation that could be differentially related to psychopathology. Furthermore, intelligence is sometimes equated with EF, raising the question of whether these cognitive constructs account for the same variance in psychopathology. Using data from the Colorado Twin Study (COTwins; 812 twins aged 14-18), we examined the phenotypic and genetic relationships among latent variables for different components of EFs (constructed from a task battery designed to assess inhibiting, updating working memory, and set-shifting abilities) and intelligence, as well as impulsivity facets (UPPS-P scales, and their relationships to self- and parent-reported psychopathology symptoms (Achenbach Youth Self-Report and Child Behavior Checklist) in adolescence. EFs and intelligence were not significantly related to any impulsivity facets. EFs showed moderate to strong positive relationships with intelligence, but EFs and intelligence displayed different associations with psychopathology. General EF was negatively related to self- and parent-reported internalizing problems at the phenotypic but not genetic level. In contrast, intelligence was positively associated with self-reported internalizing problems and negatively associated with parent-reported externalizing at the phenotypic and genetic levels. Finally, negative urgency was positively phenotypically and genetically related to both self- and parent-reported internalizing and externalizing problems. Overall, these results suggest that EFs, intelligence, and impulsivity are differentially related to internalizing and externalizing psychopathology at the phenotypic and genetic levels in adolescence.

Grant Support: This research was supported by NIH grants MH016880 and DA046413.

\section{Determining the Stability of Genome-Wide Factors in BMI Between Ages 40 to 69 Years}

Amanda Elswick Gentry ${ }^{1}$, Nathan A Gillespie ${ }^{1-2}$,

Robert M Kirkpatrick ${ }^{1}$, Hermine $\mathrm{H} \mathrm{Maes}^{3}$,

Chandra A Reynolds ${ }^{4}$, Ravi Mathur ${ }^{5}$, Kenneth S Kendler ${ }^{1}$,

Roseann E. Peterson ${ }^{1} \&$ Bradley T. Webb ${ }^{1,5}$

${ }^{1}$ Virginia Institute for Psychiatric and Behavior Genetics, Department of Psychiatry, Virginia Commonwealth University, Richmond VA, USA

${ }^{2}$ QIMR Berghofer Medical Research Institute, Herston, Queensland 4006, Australia

${ }^{3}$ Virginia Institute for Psychiatric and Behavior Genetics, Department of Human and Molecular Genetics \& Massey Cancer Center, Virginia Commonwealth University, Richmond VA, USA

${ }^{4}$ Department of Psychology, University of California, Riverside CA 92521

${ }^{5}$ GenOmics, Bioinformatics, and Translational Research Center, Biostatistics and Epidemiology Division, RTI International, Research Triangle Park, NC, USA

\section{Keywords BMI, GWAS, Genomic SEM, Longitudinal, Genetic}

Genome-wide association studies (GWAS) have successfully identified common variants associated with BMI. However, the stability of genetic variation influencing BMI from midlife and beyond is unknown. By analyzing BMI data collected from 165,717 men and 193,073 women from the UKBiobank, we performed BMI GWAS on six independent five-year age intervals between 40 and 73 years. We then applied genomic structural equation modeling (gSEM) to test competing hypotheses regarding the stability of genetic effects for BMI. LDSR genetic correlations between BMI assessed between ages 
40 to 73 were all very high and ranged 0.89 to 1.00 . Genomic structural equation modeling revealed that genetic variance in BMI at each age interval could not be explained by the accumulation of any age-specific genetic influences or autoregressive processes. Instead, a common set of stable genetic influences appears to underpin variation in BMI from middle to early old age in men and women alike.

\section{Biometric Analysis of Within-Person Flynn Effects}

Evan J. Giangrande ${ }^{1}$, Sean R. Womack ${ }^{1}$, Ramona Weber ${ }^{1}$, Christopher R. Beam ${ }^{2}$, Deborah Finkel ${ }^{3,4}$, Deborah W. Davis', Eric Turkheimer ${ }^{1}$

${ }^{1}$ Department of Psychology, University of Virginia, Charlottesville, Virginia, USA

${ }^{2}$ Department of Psychology, University of Southern California, Los Angeles, California, USA

${ }^{3}$ Department of Psychology, Indiana University Southeast, New Albany, Indiana, USA

${ }^{4}$ Institute for Gerontology, Jönköping University, Jönköping, Sweden ${ }^{5}$ Department of Pediatrics, University of Louisville School of Medicine, Louisville, Kentucky, USA

Keywords Flynn Effect, Multilevel modeling, Cognitive development, Louisville Twin Study

The systematic rise in cognitive ability scores across generations, known as the Flynn Effect (FE), is typically documented as a between-subjects phenomenon, either as gains in mean scores across cohorts (cohort effects) or sudden decreases in mean score upon test re-standardization (test version effects). The FE may also operate within individuals, boosting cognitive growth above what would be expected from age-related cognitive development. However, only two previous studies have examined within-person FEs (e.g., O'Keefe \& Rodgers, 2017), and no study has analyzed the FE using a geneticallyinformed approach. In the present study, we leveraged the unique data structure of the Louisville Twin Study (longitudinal IQ data on twins; 40 years of data collection; three WISC versions) to investigate the FE across ages 7-15 years (291 monozygotic pairs, 298 dizygotic pairs). Multilevel latent growth curve analyses revealed clear evidence of cohort and test version effects between twin pairs, and also FEs on within-person cognitive growth across age, with gains equaling approximately three IQ points per decade. Results of biometric analyses indicated that individual-level sensitivity to the FE on FSIQ, VIQ, and PIQ were associated with substantial proportions of additive genetic, shared environmental, and non-shared environmental variance. For FSIQ, A = .64, C $=.26$, and $\mathrm{E}=.10$. Implications for understandings of the FE, as well as gene-environment interplay on cognitive development, are discussed.

\section{References}

O'Keefe, P. \& Rodgers, J.L. (2017). Double decomposition of level-1 variables in multilevel models: An analysis of the Flynn Effect in the NSLY data. Multivariate Behavioral Research.

Grant Support: National Institute on Aging (R01AG063949-01 and R03AG048850-01).

\section{The Role of Genetics in Neurodevelopmental Disorders and Their Comorbidity with Disruptive, Impulse Control and Conduct Disorders During Childhood and Adolescence. A Meta-analysis}

Agnieszka Gidziela ${ }^{1}$, Yasmin Ahmadzadeh $^{2}$, Giorgia Michelini ${ }^{3}$, Andrea Allegrini ${ }^{4}$, Angelica Ronald ${ }^{5}$, Robert Plomin ${ }^{2}$, Kaili Rimfeld ${ }^{2}$ \& Margherita Malanchini ${ }^{1,2}$

${ }^{1}$ School of Biological and Chemical Sciences, Queen Mary University of London

${ }^{2}$ Social, Genetic and Developmental Psychiatry Centre, King's College London

${ }^{3}$ UCLA Semel Institute for Neuroscience, Division of Child \& Adolescent Psychiatry

${ }^{4}$ Division of Psychology and Language Sciences, University College London

${ }^{5}$ Department of Psychological Sciences, Birkbeck University of London

Keywords Meta-analysis, Neurodevelopment, Disruptive, Heritability, Comorbidity

A systematic understanding of the aetiology of neurodevelopmental disorders (NDDs) and of their comorbidities during childhood and adolescence remains incomplete. A disproportionate number of studies have focused on autism spectrum disorder (ASD) and attention deficit/hyperactivity disorder (ADHD), but other neurodevelopmental conditions have been overlooked. With three core aims, this meta-analysis bridges current gaps in our knowledge. First, we obtained meta-analytic estimates for the relative contribution of genetic and environmental influences to all NDDs categories defined in the DSM-5. Second, we meta-analysed estimates of the genetic and environmental overlap between NDDs. Third, we examined the comorbidity between NDDs and disruptive, impulse control and conduct disorders (DICCs). A total of 8,040 studies were screened by two independent reviewers, which yielded 293 eligible studies. We performed preliminary random-effects, multilevel metaanalyses, considering family-based and SNP-based estimates separately. Family-based designs yielded a grand heritability estimate for NDDs of $0.66(\mathrm{SE}=0.03)$, ranging from $0.62(0.04)$ for Specific Learning Disorder to 0.76 (0.11) for ASD; SNP-based studies yielded an estimate of $0.19(0.03)$. The genetic correlation among NDDs was $0.41(0.14)$ for family-based studies and $0.39(0.19)$ for SNP-based ones. The genetic correlation between NDDs and DICCs was 0.60 (0.18) for family-based designs. This meta-analysis extends our knowledge of NDDs in several directions. First, it provides a holistic view of genetic and environmental contributions to all NDDs and to their comorbidities over development. Second, it identifies clear gaps in the literature which point the way to future research. Third, it equips clinicians and educators with crucial new knowledge.

Grant Support: AB is funded by a School of Biological and Chemical Sciences Departmental PhD scholarship awarded to MM. 


\section{Determining the Stability of Genome-Wide Factors in BMI Between Ages 40 to 69 Years}

Nathan A Gillespie ${ }^{1-2}$, Amanda Elswick Gentry ${ }^{1}$,

Robert M Kirkpatrick ${ }^{1}$, Hermine H Maes ${ }^{3}$, Chandra A Reynolds ${ }^{4}$, Ravi Mathur ${ }^{5}$, Kenneth S Kendler ${ }^{1}$,

Roseann E. Peterson ${ }^{1} \&$ Bradley T. Webb ${ }^{1,5}$

${ }^{1}$ Virginia Institute for Psychiatric and Behavior Genetics, Department of Psychiatry, Virginia Commonwealth University, Richmond VA, USA

${ }^{2}$ QIMR Berghofer Medical Research Institute, Herston, Queensland 4006, Australia

${ }^{3}$ Virginia Institute for Psychiatric and Behavior Genetics, Department of Human and Molecular Genetics \& Massey Cancer Center, Virginia Commonwealth University, Richmond VA, USA

${ }^{4}$ Department of Psychology, University of California, Riverside CA 92521

${ }^{5}$ GenOmics, Bioinformatics, and Translational Research Center, Biostatistics and Epidemiology Division, RTI International, Research Triangle Park, NC, USA

\section{Keywords BMI, GWAS, Genomic SEM, Longitudinal, Genetic}

Genome-wide association studies (GWAS) have successfully identified common variants associated with BMI. However, the stability of genetic variation influencing BMI from midlife and beyond is unknown. By analyzing BMI data collected from 165,717 men and 193,073 women from the UKBiobank, we performed BMI GWAS on six independent five-year age intervals between 40 and 73 years. We then applied genomic structural equation modeling (gSEM) to test competing hypotheses regarding the stability of genetic effects for BMI. LDSR genetic correlations between BMI assessed between ages 40 to 73 were all very high and ranged 0.89 to 1.00 . Genomic structural equation modeling revealed that genetic variance in BMI at each age interval could not be explained by the accumulation of any age-specific genetic influences or autoregressive processes. Instead, a common set of stable genetic influences appears to underpin variation in BMI from middle to early old age in men and women alike.

\section{The Genetic and Environmental Etiology of Weighted Gene Co-Expression Network Module Eigengenes in Middle Age Sample of Male Twins}

\author{
Nathan A Gillespie ${ }^{1-2}$, Tyler R. Bell ${ }^{3-4}$, Gentry C Hearn ${ }^{5}$, \\ Jonathan L. Hess ${ }^{5}$, Ming T. Tsuang ${ }^{4}$, Carol E. Franz ${ }^{3-4}$, \\ Stephen J. Glatt ${ }^{5}$ \& William S. Kremen ${ }^{3-4}$ \\ ${ }^{1}$ Virginia Institute for Psychiatric and Behavior Genetics, Virginia \\ Commonwealth University, VA, USA \\ ${ }^{2}$ QIMR Berghofer Medical Research Institute, Herston, Queensland \\ 4006, Australia \\ ${ }^{3}$ Center for Behavior Genetics of Aging, University of California San \\ Diego, La Jolla, CA, USA \\ ${ }^{4}$ Department of Psychiatry, University of California San Diego, La \\ Jolla, CA, USA \\ ${ }^{5}$ Department of Psychiatry and Behavioral Sciences, SUNY Upstate \\ Medical University, Syracuse, NY, USA
}

Keywords Weighted gene co-expression network analysis, Module eigengene, Twin gene

Weighted gene co-expression network analysis (WGCNA) is a wellvalidated multivariate network-based analytic method for identifying genes driving complex traits. Despite their increasing application, the sources of individual differences in network module eigengenes have never been empirically established. WGCNA works by classifying gene sets into 'network modules' based on their particular patterns of gene expression (GExp) and connectivity. These patterns are typically summarized with a single quantitative metric or module eigengene (ME), which is a quantitative summary of the correlated expression and connectedness across all genes in a module. Using a sample of 646 early old age male twins from the Vietnam Era Twin Study of Aging with GExp data based on blood lymphocytes, our aim was to explore the genetic and environmental etiology of WGCNA-derived MEs. GExp data were filtered to have $>1$ CPM expression in at least $50 \%$ of the sample, adjusted for batch effects, and log-transformed before running WGCNA. Full Informative Maximum Likelihood biometric genetic models were then fitted to the first 30 MEs. Results were adjusted for covariates including age, ethnicity, site, storage time and comorbid disease. Across all MEs, familial aggregation was significant and was explained by varying proportions of additive genetic (ranging 37-63\%) and shared environmental (ranging 26-52\%) risks. Detailed results and implications along with exploratory associations with mild cognitive impairment will be presented.

Grant Support: This work was assisted through funding from the National Institute on Aging [R01 AG054002, R01 AG060470, R01 AG022381, R01 AG050595].

\section{The Costs of Victimization on Job Retention}

B.M.A. Gonggrijp ${ }^{1,2}$, S. van de Weijer ${ }^{2}$, J. van Dongen ${ }^{1}$, E.M.A. Slob ${ }^{3,4}$, C. Bijleveld ${ }^{2}$, D.I. Boomsma ${ }^{1}$

${ }^{1}$ Department of Biological Psychology, Netherlands Twin Register, Vrije Universiteit Amsterdam, Amsterdam, The Netherlands

${ }^{2}$ Netherlands Institute for the Study of Crime and Law Enforcement (NSCR), Amsterdam, The Netherlands

${ }^{3}$ Department of Respiratory Medicine, Amsterdam University Medical Centers, DE Amsterdam, The Netherlands

${ }^{4}$ Department of Paediatric Pulmonology, Amsterdam University Medical Centers, Amsterdam, The Netherlands

Keywords Victimization, Unemployment, Occupation, Discordant twin analysis

During the past decades, it has been increasingly recognized that victimization can be a life-altering experience. Previous research has shown that victims of crime and accidents report less favorable life outcomes than non-victims. This is so across a host of such lifeoutcomes, such as mental health, relationship formation, and employment. However, due to confounding it has been hard to causally link such outcomes to victimization experience and thus determining what exactly the 'costs' of victimization are. The present study is based on data from eight surveys collected by the Netherlands Twin Register (NTR) between 2000-2009. All surveys included information about unemployment, working hours and victimization. Participants with the age of 15-65 were selected. This research aims to determine the cost of victimization on job retention by using longitudinal data in an unmatched cohort analysis $(\mathrm{N}=18,296)$ and a discordant twin design $(\mathrm{N}=1440)$. In the cohort analysis we see an overall decrease in unemployment for victims compared to non-victims. In the discordant twins however, there is an increase in unemployment if the event was 0-5 years ago. Looking at working fulltime versus parttime we see a decrease in working parttime for individuals who experienced a crime $0-5$ years ago compared to nonvictims. When the event was longer ago $(5+$ years $)$ we see an 
increase in working part-time among the victims. The same results were found in both the $\mathrm{MZ}$ as $\mathrm{DZ}$ discordant twin pairs.

\section{Liquor Legacies: The Impact of Parental Psychopathology and Young Adult Depression on Alcohol Use}

Rachel Good ${ }^{1}$, Yoo Ri Hwang ${ }^{1}$, S. Mason Garrison ${ }^{1}$

${ }^{1}$ Department of Psychology, Wake Forest University, Winston-Salem, NC USA

Keywords Alcoholism, Psychopathology, Depression, Sibling comparison

Children of alcoholics have increased risks for developing alcohol abuse or dependence. Unhealthy relationships with alcohol may affect daily functioning and increase risks for other health and mental health related issues. These consequences affect subsequent generations (Chassin, et al. 1999). Therefore, we aim to understand whether parental alcohol abuse actually affects an individual's alcohol use or reflects between family effects (such as shared environment or genetics).

Using the National Longitudinal Survey of Youth 1979, we examined the impact of parental psychopathology and young adult depression on alcohol use at age 60 . Results differed by whether we employed classic regression approaches versus when compared siblings using the discordant kinship model (Garrison \& Rogers, 2016). The covariate approach suggested that all variables of interest were predictive of later alcohol use, whereas the sibling comparisons found that no such effect. In other words, the relationship between parental psychopathology and young adult depression on alcohol use appears to be a between family effect, suggesting that neither directly causes alcohol use at age 60 .

References

Chassin, L., Pitts, S. C., DeLucia, C., \& Todd, M. (1999). A longitudinal study of children of alcoholics: predicting young adult substance use disorders, anxiety, and depression. Journal of abnormal psychology

Garrison, S. M., \& Rodgers, J. L. (2016). Casting doubt on the causal link between intelligence and age at first intercourse: A crossgenerational sibling comparison design using the NLSY. Intelligence

Grant Support: N/A.

\section{Use of GWAS by Subtraction in Lieu of Covariates: The Expected and Unexpected}

\author{
Katrina L. Grasby ${ }^{1}$ \\ ${ }^{1}$ QIMR Berghofer Medical Research Institute, Brisbane, QLD, \\ Australia
}

Keywords GWAS, GWAS-by-subtraction, Method comparison, ENIGMA

GWAS summary statistics are extremely useful for a myriad of follow up analyses, which are made possible through various open-access programs, including genomicSEM ${ }^{1}$. With large collaborations of researchers pooling their data and making GWAS results freely available, there is an ever increasing amount of data available to improve our understanding of genotype and phenotype. In a recent GWAS of the cortex ${ }^{1}$ by the ENIGMA consortium, in excess of 11 TB of data were uploaded to a central server for analysis. However, approximately half of the data was essentially a duplicate model that included a global control measure. I explored if GWAS-by-subtraction $^{2}$ would provide equivalent results, thus requiring half the GWAS and half the data storage for future work that implement similar protocols. From the cortical results, GWAS-by-subtraction was run "subtracting" total surface area from the genetic effects on the surface area of the precentral sulcus. Results were compared with the original GWAS that included total surface area as a covariate in the regression. The GWAS-by-subtraction results genetically correlated 1.0 with the original summary statistics. However, only a third of the original loci were significant. While most loci were slightly reduced in significance, one very strong signal was reduced by a large amount. Respecifying the model solved the discrepancy in results.

References

${ }^{1}$ Grasby K.L. et al. The genetic architecture of the human cerebral cortex. Science 367.6484 (2020)

${ }^{2}$ Demange, P.A., Malanchini, M., Mallard, T.T. et al. Investigating the genetic architecture of noncognitive skills using GWAS-by-subtraction. Nat Genet 53, 35-44 (2021)

Grant Support: Australian NHMRC APP1173025.

\section{An Examination of Early Socioeconomic Status and Neighborhood Disadvantage as Independent Predictors of Antisocial Behavior: A Longitudinal Adoption Study}

Shelley A. Gresko ${ }^{1,2 *}$, Laura Hink ${ }^{1,2 *}$, Robin P. Corley ${ }^{2}$, Elizabeth Muñoz ${ }^{3}$, Chandra A. Reynolds ${ }^{4}$, Soo Hyun Rhee ${ }^{1,2}$

${ }^{1}$ Department of Psychology and Neuroscience, University of Colorado Boulder, USA

${ }^{2}$ Institute for Behavioral Genetics, University of Colorado Boulder, USA

${ }^{3}$ Department of Human Development and Family Sciences, University of Texas Austin, USA

${ }^{4}$ Institute for Integrative Genome Biology, University of California Riverside, USA

*Denotes co-first authors

Keywords Antisocial behavior, Socioeconomic status, Neighborhood disadvantage, Adoption study, Longitudinal

Prior research consistently shows that low socioeconomic status (SES) and neighborhood disadvantage (ND) predict antisocial behavior (ASB). However, few studies have addressed whether SES and ND are independent predictors of ASB, or the mechanisms of these associations. The present study had the following aims: (1) determine whether SES and neighborhood disadvantage are independent predictors of ASB, (2) test whether associations are primarily influenced via environmental mediation or passive gene environment correlation, (3) examine sex differences in associations. Data from the Colorado Adoption Project was used. SES (parental education and occupation) was assessed from birth to age 1. A neighborhood deprivation composite was created for familial address at birth to age 1, using 7 census tract indicators. ASB was assessed by parents (via the externalizing scale of the Child Behavior Checklist) and teachers (via the externalizing scale of the Teacher Report Form) from age 7-16. Results indicated that, in adoptees, parental SES did not 
significantly predict ASB. After controlling for ND, SES was an independent, negative predictor of ASB in nonadoptees. In nonadopted girls, as SES increased, there was significantly less change in ASB. Overall, associations were not significantly different by sex. Although the association between SES and ASB was greater in nonadoptees than adoptees, there was not a significant group difference. The lack of significant group difference may be due to low power. Future analyses will examine if patterns of associations differ between parent- and teacher- reported ASB.

Grant Support: National Institutes Health grants HD010333, DA035804, DA011015, and AG046938.

\section{Alzheimer's Disease Polygenic Scores Predict Changes in Executive Function and Episodic Memory Across 12 years in Late Middle Age}

Daniel E. Gustavson ${ }^{1,2}$, Chandra A. Reynolds ${ }^{3}$, Timothy J. Hohman ${ }^{2}$, Angela L. Jefferson ${ }^{2}$, Matthew S. Panizzon ${ }^{4}$, Michael C. Neale ${ }^{5}$, and Carol E. Franz ${ }^{4}$, William S. Kremen ${ }^{4,6}$

${ }^{1}$ Department of Medicine, Vanderbilt Genetics Institute, Vanderbilt University Medical Center, Nashville, Tennessee, USA

${ }^{2}$ Vanderbilt Memory and Alzheimer's Center, Vanderbilt University Medical Center, Nashville, Tennessee, USA

${ }^{3}$ Department of Psychology, University of California, Riverside, California, USA

${ }^{4}$ Department of Psychiatry, University of California, San Diego, La Jolla, California, USA

${ }^{5}$ Virginia Institute for Psychiatric and Behavior Genetics, Virginia Commonwealth University, Richmond, VA

${ }^{6}$ VA Center of Excellence for Stress and Mental Health, La Jolla, California, USA

Keywords Polygenic risk score, Alzheimer's disease, Neuropsychology, Cognitive aging

Genome-wide association studies (GWAS) are instrumental in quantifying genetic influences underlying Alzheimer's disease (AD), and applications of polygenic scores (PRS) derived from GWAS have already shed light on early disease pathology. What remains unclear is how AD-PRS relate to objectively measured cognitive changes across midlife. We examined 1388 men in the Vietnam Era Twin Study of Aging (VETSA) who were cognitively normal at their first assessment and had up to 3 cognitive assessments across 10-12 years (mean ages 56, 62, and 68). Latent growth models of executive function were based on 6 tasks spanning inhibition, shifting, and working memory subdomains and growth models of episodic memory were based on 7 subtests from the Logical Memory, Visual Reproductions, and California Verbal Learning tests. The AD-PRS was based on Kunkle et al. (2019), $p<5 \times 10^{-8}$ threshold. Results demonstrated that baseline executive function $(r=.11)$ and memory $(r=-.07)$ were not associated with AD-PRS. Importantly, individuals with higher AD-PRS had sharper declines in executive functioning $(r=-.38,95 \%$ CI $[-.64,-.14])$ and episodic memory $(r=-.20,95 \%$ CI $[-.37,-.03])$ from midlife to early old age. Associations were driven by ApoE and were nonsignificant after excluding the ApoE region from the AD-PRS. These findings highlight the importance of considering executive function changes in midlife, especially for predicting disease progression and AD-related biology. Executive functions are one of the first cognitive abilities to decline in midlife in normal aging. This study is among the first to demonstrate that this early decline also relates to $\mathrm{AD}$ genetic influences.
Grant Support: National Institutes of Health, National Institute on Aging grants: R03 AG065643; R01 AG050595; R01 AG022381.

\section{Associations Between the Subdomains of Negative Symptoms in the General Population and Genome- Wide Polygenic Scores for Major Depressive Disorder and Schizophrenia}

Laura Havers $^{1}$, Alastair Cardno ${ }^{2}$, Daniel Freeman ${ }^{3}$, Angelica Ronald ${ }^{1}$ ${ }^{1}$ Department of Psychological Sciences, Birkbeck, University of London, London, UK

${ }^{2}$ School of Medicine, University of Leeds, Leeds, UK. ${ }^{3}$ Department of Psychiatry, University of Oxford, Oxford, UK

Keywords Psychosis continuum, Genome-wide polygenic scores, Subdomain-specificity, Confirmatory factor analysis

Negative symptoms predict adverse outcomes within psychotic disorders, in individuals at high-risk for psychosis, and in young people in the community. Accumulating evidence suggests a 5-factor latent structure of these symptoms, but little is known about their underlying structure outside of clinical and high-risk samples. It is also unknown which aspects of negative symptoms show the strongest genetic links with clinically diagnosed depression and schizophrenia. We used confirmatory factor analysis to test the structure of parent-reported negative symptoms at 3 ages in adolescence and emerging adulthood (mean ages 16.32, SD 0.68; 17.06, SD 0.88; 22.30, SD 0.93) in the Twins Early Development Study $(N=1468-5177)$. We assessed associations between the negative symptom subdomains and genomewide polygenic scores (GPS) for major depressive disorder (MDD) and schizophrenia. A 5-factor model of flat affect, alogia, avolition, anhedonia and asociality provided the best fit at each age and was invariant over time. Associations were observed between MDD GPS with avolition, flat affect, anhedonia and asociality, and between schizophrenia GPS with avolition and flat affect. We showed that a 5-factor structure of negative symptoms, previously identified in clinical samples, is present from ages 16 to 22 years in the community. Avolition was most consistently associated with known common genetic architecture underlying MDD and schizophrenia, and alogia was least associated. These findings highlight the value of dissecting negative symptoms into psychometrically derived subdomains and may offer insights into early manifestation of genetic risk for MDD and schizophrenia.

Grant Support: Medical Research Council grant G1100559 to AR. TEDS is funded by Medical Research Council grant MR/M021475/1 to Robert Plomin. LH was funded by an ESRC PhD studentship.

\section{Can Interventions Mitigate Genomic Liability for Obesity? Using Causal Inference Based Mediation Analyses in Genetically-Sensitive Studies}

Moritz Herle ${ }^{1}$, Andrew Pickles ${ }^{1}$, Bianca De Stavola ${ }^{2}$

${ }^{1}$ Department of Biostatistics \& Health Informatics, Institute of Psychology, Psychiatry and Neuroscience, Kings College London, UK

${ }^{2}$ Population, Policy \& Practice, UCL Great Ormond Street Institute of Child Health, University College London, London, UK

Keywords Causal inference, Obesity, Physical activity, BMI, Polygenic scores 
Polygenic scores are now commonly available in longitudinal cohort studies, leading to their integration into epidemiological research. In this work, our aim is to explore how polygenic scores can be used as exposures in causal inference based methods, specifically mediation analyses. We propose to apply the interventional disparity measure approach to estimate the extent to which the direct effect from an exposure to an outcome could be mitigated by a potential intervention on an intermediate mediator. In order to do this this, we use the interventional disparity measure approach, which allows us to compare the adjusted total causal effect of an exposure on an outcome, with the effect that would have occurred had we intervened on a intermediate mediator. As an example, we analyze data from two UK cohorts, the Avon Longitudinal Study of Parents and Children (ALSPAC) and the Millennium Cohort Study. In both, the exposure is genetic liability for obeisty (indicated by a polygenic risk score for BMI), the outcome is late childhood/early adolescent BMI, and the mediator and potential intervention target is physical activity, measured intermediately between exposure and outcome. Our results suggest that a potential intervention on child physical activity can mitigate some of the genetic liability for childhood obesity. Causal inference based methods can be beneficial as they ask researchers to state specific hypotheses and ground their research in the context of potential interventions, linking these investigations directly to clinical applications and wider public health.

Grant Support: This research was supported by a fellowship from the Medical Research Council UK (MR/T027843/1) awarded to Moritz Herle.

\section{Constitutional Thinness and Anorexia Nervosa Differ on a Genomic Level}

Christopher Hübel ${ }^{1,2,3,4} *$, Mohamed Abdulkadir ${ }^{5,6 *}$, Moritz Herle ${ }^{7}$, Alish B. Palmos ${ }^{1,2}$, Ruth J.F. Loos ${ }^{9}$, Gerome Breen ${ }^{1,2}$,

Nadia Micali $^{5,6,9 \dagger}$ \& Cynthia M. Bulik ${ }^{10,11 \uparrow}$

${ }^{1}$ Social, Genetic \& Developmental Psychiatry Centre, Institute of Psychiatry, Psychology \& Neuroscience, King's College London, UK ${ }^{2}$ National Institute for Health Research (NIHR) Maudsley Biomedical Research Centre at South London and Maudsley NHS Foundation Trust, London, UK

${ }^{3}$ National Centre for Register-based Research, Aarhus Business and Social Sciences, Aarhus University, Aarhus, Denmark

${ }^{4}$ Department of Medical Epidemiology and Biostatistics, Karolinska Institutet, Stockholm, Sweden

${ }^{5}$ Department of Pediatrics Gynaecology and Obstetrics, Faculty of Medicine, University of Geneva, Geneva, Switzerland

${ }^{6}$ Department of Psychiatry, Faculty of Medicine, University of Geneva, Geneva, Switzerland

${ }^{7}$ Department of Biostatistics \& Health Informatics, Institute of Psychiatry, Psychology \& Neuroscience, King's College London, UK

${ }^{8}$ Charles Bronfman Institute for Personalized Medicine, Icahn School of Medicine at Mount Sinai, New York, New York, USA

${ }^{9}$ Great Ormond Street Institute of Child Health, University College London, London, UK

${ }^{10}$ Department of Psychiatry, University of North Carolina at Chapel Hill, Chapel Hill, NC, USA

${ }^{11}$ Department of Nutrition, University of North Carolina at Chapel Hill, Chapel Hill, NC, USA

*contributed equally to this work

$\dagger$ shared last authorship
Keywords Avon Longitudinal Study of Parents and Children (ALSPAC), Polygenic scores, Genetic correlations, Latent class growth analysis

Constitutional thinness (CT) and anorexia nervosa (AN) are both phenotypes that present with low body weight defined as a BMI below $18.5 \mathrm{~kg} / \mathrm{m}^{2}$. However, individuals with anorexia nervosa additionally report a fear of weight gain and engage in behaviors leading to weight loss not reported or seen in constitutional thinness. Body composition regulation and anorexia nervosa are both heritable complex traits. Currently, it is unclear how constitutional thinness differs from anorexia nervosa on a genomic level. We calculated genetic correlations between constitutional thinness and anorexia nervosa and between constitutional thinness and traits that are genetically correlated with anorexia nervosa, to investigate potential similarities and differences. Additionally, we identified individuals with constitutional thinness in the Avon Longitudinal Study of Parents and Children (ALSPAC) and performed polygenic score analyses. Our results suggest that, in contrast to anorexia nervosa, attention deficit hyperactivity disorder $\left(r_{\mathrm{gAN}}=0.02\right.$ vs. $\left.r_{\mathrm{gCT}}=-0.24\right)$ and alcohol dependence $\left(r_{\mathrm{gAN}}=0.07\right.$ vs. $\left.r_{\mathrm{gCT}}=-0.44\right)$ are significantly genetically correlated with constitutional thinness. A higher polygenic score for posttraumatic stress disorder is associated with an increased risk of constitutional thinness in the ALSPAC cohort (OR 1.27; $Q=$ $0.03)$ whereas posttraumatic stress disorder shows no genetic correlation with anorexia nervosa $\left(r_{\mathrm{g}}=-0.02\right)$. In conclusion, our research proposes that even though both anorexia nervosa and constitutional thinness are marked by low BMI, they differ on a genomic level.

Grant Support: Biomedical Research Centre at South London and Maudsley NHS Foundation Trust and King's College London, GSTT Charity (TR130505), Maudsley Charity (980), Medical Research Foundation (ref: MR/R004803/1), Wellcome (Grant ref: 102215/2/13/ 2 and 217065/Z/19/Z), NIMH (R21 MH115397; R01MH120170; R01MH119084; R01MH118278; U01 MH109528), Vetenskapsrådet (award: 538-2013-8864), Lundbeck Foundation (Grant no. R2762018-4581), Medical Research Council UK (MR/T027843/1).

\section{Major Depressive Disorder and Lifestyle: Pleiotropic Effects in Bivariate Genetic Analyses of Extended Twin Pedigrees}

Floris Huider ${ }^{1}$, Brenda W.J.H. Penninx ${ }^{2}$, Yuri Milaneschi ${ }^{2}$, Matthijs van der Zee ${ }^{1}$, Eco J. C. de Geus ${ }^{1}$, Quinta Helmer ${ }^{1}$,

\& Dorret I. Boomsma ${ }^{1}$

${ }^{1}$ Department of Biological Psychology, Vrije Universiteit, Amsterdam, The Netherlands

${ }^{2}$ Department of Psychiatry, Amsterdam UMC, Vrije Universiteit, Amsterdam, The Netherlands

Keywords Depression, Pleiotropy, Lifestyle, Extended twin pedigree, Variance decomposition

Major Depressive Disorder (MDD) is a prevalent, burdensome and heterogeneous disorder with a complex etiology of genetic and environmental determinants (Kendall et al., 2021). In recent years, evidence has accumulated with regard to the ubiquity of pleiotropy across the genome (Watanabe et al., 2019), and shared genetic etiology is thought to play a large role in the widespread comorbidity among psychiatric disorders and risk factors (van Rheenen, Pevrot, Schork, Lee, \& Wray, 2019). The majority of recent methods investigate pleiotropy by estimating genetic correlation from Genome-Wide Association (GWA) summary statistics, but such estimates can also be 
derived from the known relatedness between genetic relatives. Here we conduct a series of bivariate genetic analyses in extended twin pedigree data on lifetime MDD and three indicators of lifestyle, namely smoking behavior, physical inactivity and Body-Mass Index, decomposing phenotypic variance and covariance into genetic and environmental components. We analyze lifetime MDD and lifestyle data in a large multigenerational dataset of $N=18,896$ individuals, containing 4882 families with two or more members and 8677 twins, using the 'Mendel' software (Lange et al., 2013). Analysis of extended twin pedigree data allows for the estimation of genetic correlation for both additive and non-additive genetic effects, as well as shared environmental effects. Quantifying the relative contribution of genetic and environmental effects on MDD liability and comorbidity with risk factors benefits our understanding of its etiological architecture.

\section{References}

Kendall, K. M., Assche, E. V., Andlauer, T. F. M., Choi, K. W., Luykx, J. J., Schulte, E. C., \& Lu, Y. (2021). The genetic basis of major depression. Psychological Medicine, 1-14.

Lange, K., Papp, J. C., Sinsheimer, J. S., Sripracha, R., Zhou, H., \& Sobel, E. M. (2013). Mendel: The Swiss army knife of genetic analysis programs. Bioinformatics, 29(12), 1568-1570.

van Rheenen, W., Peyrot, W. J., Schork, A. J., Lee, S. H., \& Wray, N. R. (2019). Genetic correlations of polygenic disease traits: From theory to practice. Nature Reviews Genetics, 20(10), 567-581.

Watanabe, K., Stringer, S., Frei, O., Umićević Mirkov, M., de Leeuw, C., Polderman, T. J. C., van der Sluis, S., Andreassen, O. A., Neale, B. M., \& Posthuma, D. (2019). A global overview of pleiotropy and genetic architecture in complex traits. Nature Genetics, 51(9), 1339-1348.

Grant Support: This work was supported by the Royal Dutch Academy for Arts and Science (KNAW) Academy Professor Award (PAH/ 6635) to DIB; the Netherlands Organization for Scientific Research (NWO 480-15-001/674) and Biobanking and Biomolecular Resources Research Infrastructure (BBMRI-NL: 184.021.007; 184.033.111).

\section{Genetic and Environmental Contributions to Stability and Change in Computer-Assessed Inhibitory Control Across the Preschool Period}

\author{
I-Tzu Hung ${ }^{1}$, Jody Ganiban ${ }^{2} \&$ Kimberly Saudino ${ }^{1}$ \\ ${ }^{1}$ Department of Psychological and Brain Sciences, Boston University, \\ Boston, MA, USA; \\ ${ }^{2}$ Department of Psychological and Brain Sciences, George Wash- \\ ington University, Washington, DC, USA.
}

\section{Keywords Inhibitory control, Flanker, Preschool, Twins}

Research exploring the etiology and development of inhibitory control (IC) in early childhood is mixed. Parent-ratings display significant heritabilities at ages 2 and 3, but game-like lab-based measures are only heritable at age 2 (Gagne \& Saudino, 2016). One possible explanation is that the lab measure reached ceiling at age 3 , hence more complex measures of IC might yield different results. The present study uses a cognitively-based Flanker task to examine the heritability of IC in a sample of 275 same-sex twin pairs $(M Z=112, D Z=163)$ assessed within one month of their 3rd, 4th, and 5th birthdays. IC displayed lowmoderate stability (ranging from .11 to .24). Longitudinal trivariate Cholesky models reveal that IC was significantly heritable at each age $\left(h^{2}\right.$ : age $3=.36$; age $4=.36$; age $\left.5=.35\right)$. No significant shared environmental influences were observed. There were genetic effects that persisted from age 3 to ages 4 and 5 , novel genetic effects emerged in age 4 and persisted to age 5, and also novel genetic effects on IC at age $5.87 \%$ of the genetic effects of age 4 were independent of age 3 , and $50 \%$ of the genetics effects at age 5 were independent of those at prior ages. The finding of significant heritability with the Flanker task at age 3 suggests that the lack of heritability for the play-based measures of IC at age 3 is methodological.

Grant Support: This research was supported by grant R01HD068435 to Dr. Saudino.

\section{Using Adopted Singletons to Partition Maternal Genetic Effects into Pre- and Post-natal Effects on Offspring Phenotype}

Liang-Dar Hwang ${ }^{1,2}$, Gunn-Helen Moen ${ }^{1,2,3,4,5}$, David M Evans ${ }^{1,2,6}$ ${ }^{1}$ Institute for Molecular Bioscience, The University of Queensland, Brisbane, Australia

${ }^{2}$ The University of Queensland Diamantina Institute, The University of Queensland, Brisbane, Australia

${ }^{3}$ Institute of Clinical Medicine, Faculty of Medicine, University of Oslo, Oslo, Norway

${ }^{4}$ Population Health Science, Bristol Medical School, University of Bristol, Bristol, United Kingdom

${ }^{5}$ K.G. Jebsen Center for Genetic Epidemiology, Department of Public Health and Nursing, NTNU, Norwegian University of Science and Technology, Trondheim, Norway

${ }^{6}$ Medical Research Council Integrative Epidemiology Unit at the University of Bristol, Bristol, United Kingdom

Keywords Adoption studies, Maternal effects, Mendelian randomization, Educational attainment, Birthweight

Maternal genetic effects can be defined as the effect of a mother's genotype on the phenotype of her offspring, independent of the offspring's genotype. Maternal genetic effects can act via the intrauterine environment during pregnancy and/or via the post-natal environment. Here we develop a new model using structural equation modelling (SEM) to partition maternal genetic effects into pre- and post-natal effects. We assume that in biological families, offspring phenotypes are influenced prenatally by their mother's genotype and postnatally by their parents' genotypes, whereas adopted individuals' phenotypes are influenced prenatally by their biological mother's genotype and postnatally by their adoptive parents' genotypes. Critically, SEM allows us to model the potentially unobserved genotypes of biological and adoptive parents as latent variables, permitting us to leverage the thousands of adopted singletons in the UK Biobank.We examine the utility and power of our model using simulations and asymptotic power calculations.We apply our model to educational attainment and birthweight, in up to 4197 adopted singletons, 969 trios, 3545 motheroffspring pairs, 1635 father-offspring pairs and 291642 singletons from the UK Biobank. Our results show expected patterns of maternal effects on offspring birthweight, but unexpected large prenatal effects on offspring educational attainment. Sensitivity analyses suggest this result may be due to adopted individuals in UK Biobank being fostered by their relatives. We conclude that our model can be used to estimate prenatal and postnatal maternal effects on offspring phenotypes, which has exciting implications for the development of pleiotropy robust Mendelian randomization approaches.

Grant Support: NHMRC Ideas Grant GNT1183074, NHMRC Project Grant GNT1157714. 


\section{Breaking Down the Components of the SES-Health Gradient with Sibling Comparisons}

\author{
Yoo Ri Hwang ${ }^{1}$, Jonathan D Trattner ${ }^{2}$, S. Mason Garrison ${ }^{1}$ \\ ${ }^{1}$ Department of Psychology, Wake Forest University, Winston Salem, \\ NC USA \\ ${ }^{2}$ Department of Interdisciplinary Studies, Wake Forest University \\ Winston-Salem, North Carolina, USA
}

Keywords Socioeconomic Status, Health, Sibling Comparison

Although the SES-health gradient is well-established (Adler, 1994), the relationship of different factors of SES with health is not. Often, the components of SES are used interchangeably without investigation and may not be explicitly stated. Further, SES-health research typically ignores familial confounding as well as intergenerational effects. To address these limitations, we compare two methodological approaches. Specifically, we examined each component of SES and its consequences in health, and compared the results from the classic regression approach with the sibling comparison method. Data is from the 1979 National Longitudinal Survey of Youth; we quantified SES as income, wealth, education levels, and occupation to predict mental and physical health at ages $40+, 50+$, and $60+$. We also included the presence of a will or a trust at age $60+$. First, we conducted regression analyses to see how SES components related to health outcomes. Then, we replicated this regression using a discordant-kinship model (Garrison \& Rogers, 2016) to distinguish within- and between-family variance. We compared the results of each approach and found that results differed. The classic approach suggested that many components were predictive of health, whereas the sibling comparisons found results that differed across components and measures of health. Specifically, fewer components were significantly predictive of health in the sibling comparison model. Further implications were discussed.

\section{References}

Adler, N. E., Boyce, T., Chesney, M. A., Cohen, S., Folkman, S., Kahn, R. L., \& Syme, S. L. (1994). Socioeconomic status and health: the challenge of the gradient. American psychologist

Garrison, S. M., \& Rodgers, J. L. (2016). Casting doubt on the causal link between intelligence and age at first intercourse: A cross-generational sibling comparison design using the NLSY. Intelligence

\section{Active by Nature, or Active by Nurture? A Twin Study of Preschoolers' Observed Motor Behavior}

\author{
Matthew R. Jamnik ${ }^{1}$, Lisabeth F. DiLalla ${ }^{2}$ \\ ${ }^{1}$ School of Psychological and Behavioral Sciences, Southern Illinois \\ University, Carbondale, Illinois, USA \\ ${ }^{2}$ Department of Family and Community Medicine, Southern Illinois \\ University School of Medicine, Carbondale, Illinois, USA
}

Keywords Twins, Early Childhood, Physical Activity, Motor Behavior

Many children today do not meet recommended guidelines for daily physical activity, yet little research has examined movement in preschoolers. It is important to study the underlying etiology of activity to better understand how to positively impact children's behaviors. We hypothesized that: (1) genetic influences would significantly contribute to preschoolers' observed motor behavior, but (2) considering that sedentary lifestyles have rapidly increased only relatively recently, environment would account for greater variance. At age 5, families took part in the Southern Illinois Twins/Triplets and Siblings Study ${ }^{1}$. During testing, parents and children completed a parent-child interaction task. Coders rated the physical activity that children exhibited during this interaction using a new coding scheme designed to measure young children's motor and sedentary behaviors ${ }^{2}$. An ADE model was tested based on intraclass correlations, $r \mathrm{MZ}=0.76, p<.001, r \mathrm{DZ}=0.20, p=$ .023 . The AE model fit best, with $66 \%$ of variance attributed to additive genetic effects and $34 \%$ attributed to non-shared environment. Thus, a new reliable coding scheme provided unbiased, observer ratings to assess young children's physical activity. Findings revealed that both genetic and environmental influences contribute to the emergence of preschoolers' observed motor behavior; however, genetic factors appear most salient.

\section{References}

${ }^{1}$ DiLalla LF, Jamnik MR (2019) The Southern Illinois Twins/ Triplets and Siblings Study (SITSS): A longitudinal study of early child development. Twin Research and Human Genetics, 22(6), 779-782.

${ }^{2}$ Jamnik MR (2021) A behavioral genetic investigation of activity levels and internalizing problems across childhood. $\mathrm{PhD}$ diss, Southern Illinois University Carbondale

Grant Support: Partial funding for this study was provided to MRJ by the Grants in Aid of Research (GIAR) program from Sigma Xi: The Scientific Research Honor Society and the Mamie Phipps Clark Research Grant from Psi Chi: The International Honor Society in Psychology.

\section{Phenotypic and Genetic Study of a Wellbeing Factor Score in the UK Biobank and the Impact of Childhood Maltreatment}

Javad Jamshidi ${ }^{1,2}$, Peter R Schofield ${ }^{1,3}$, Justine M Gatt ${ }^{1,2, *}$ \& Janice M Fullerton ${ }^{1,3, *}$

${ }^{1}$ Neuroscience Research Australia, Sydney, NSW, Australia

${ }^{2}$ School of Psychology, University of New South Wales, Sydney, NSW, Australia

${ }^{3}$ School of Medical Sciences, University of New South Wales, Sydney, NSW, Australia

\section{*Joint senior authors}

Keywords Wellbeing, Factor analysis, Polygenic score, Genome-wide association study (GWAS), Childhood maltreatment

Wellbeing is an important aspect of mental health, distinct from the absence of mental illness. Wellbeing is moderately heritable, and large-scale genome-wide association studies (GWAS) have identified specific wellbeing-related variants. While there are various subcomponents of wellbeing, measured with different instruments and assessing different facets of the same spectrum, they are usually correlated. Therefore, a score derived from multiple questions has potential to capture a more stable and heritable wellbeing phenotype, thereby increasing power for genetic and phenotypic studies. We employed the population-based UK Biobank baseline dataset of selfreport questionnaires to create a wellbeing factor score derived from indices of: happiness and satisfaction with family, friendship, financial and health; using principal component analysis. A GWAS was performed in 129,237 Caucasian participants using the derived wellbeing score, followed by polygenic profiling in an independent sample $(n=23,703)$ to predict the wellbeing score and correlated 
phenotypes. The wellbeing score, its subcomponents and some negative indicators of mental health (including neuroticism, depressive symptoms, and loneliness) were compared in terms of phenotypic and genetic correlations. Lastly, the impact of childhood maltreatment on the wellbeing score was investigated. We identified four genomewide significant GWAS hits for wellbeing. SNP heritability was $\sim 8.6 \%$, higher than each subcomponent. The wellbeing score had the highest phenotypic and genetic correlation with mental health phenotypes compared to individual wellbeing items, except for neuroticism on happiness. Additionally, childhood trauma significantly reduced wellbeing scores, with a moderate negative genetic correlation $(\sim-0.56)$. Thus, a wellbeing index can elucidate phenotypic and genetic relationships with mental health and is moderated by trauma.

Grant Support: JJ was supported by the UNSW Scientia PhD Scholarship Scheme.

\section{Estimating SNP-Based Heritability of Complex Traits in Diverse Ancestry: Method Comparisons}

Seon-Kyeong Jang ${ }^{1}$, Saonli Basu ${ }^{2}$, Zhatong Lin $^{2}$, Scott Vrieze ${ }^{1}$, Trans-Omics Precision Medicine

${ }^{1}$ Department of Psychology, University of Minnesota, Minneapolis, MN, USA

${ }^{2}$ Department of Biostatistics, University of Minnesota, Minneapolis, MN, USA

Keywords Heritability, Admixed, Population structure, Linear mixed model, HE regression

SNP-based heritability $\left(\mathrm{h}_{\text {SNP }}^{2}\right)$ indicates the degree to which a set of genotyped variants and their tagging variants account for phenotypic variance of a given trait. It can be estimated by linear mixed model or Haseman-Elston (HE) regression using unrelated individuals. To date, the existing methods can only be applied to population with relatively little recent admixture. Extending the methods to the genomes of diverse ancestry enrich our understanding of genetic etiology of complex traits and potentially improve power by incorporating larger sample. Here we are considering three approaches to estimating $h^{2}{ }_{\text {SNP }}$ of two anthropometric traits (height and BMI) and two smoking traits (age of smoking initiation and cigarettes per day) in a sample with population substructure; (1) linear mixed model with PC adjustments (GCTA-REML), (2) HE regression with PC adjustments in a secondorder estimating equation (Adj-HE), and (3) HE regression with kinship estimates adjusted for PCs (PC-relate-HE). We used TOPMed whole-genome sequences of 43,724 and $>26,000$ unrelated samples for anthropometric and smoking traits, respectively, of which $30 \%$ consisted of non-European ancestry. Using variants common in European, African and Hispanic admixed ancestries, $\mathrm{h}^{2}$ SNP was estimated similarly across two methods (GCTA-REML and Adj-HE): about 0.40 and 0.18 (SE 0.008-0.01) for height and BMI, and 0.04 and 0.08 (SE 0.013) for age of smoking initiation and cigarettes per day. We further test the performance of all three methods in admixed populations separately and consider the influence of ancestry-specific common variants along with variance differences among cohorts.

\section{The Predictive Capacity of Psychiatric and Psychological Polygenic Risk Scores for Distinguishing Cases in a Child and Adolescent Psychiatric Sample from Controls}

Arija G. Jansen, ${ }^{1,2}$ Philip R. Jansen, ${ }^{1,2,3}$ Jeanne E. Savage, ${ }^{1}$ Julia Kraft, ${ }^{4,5}$, Nora Skarabis, ${ }^{4}$ Tinca J. C. Polderman, ${ }^{1,6}$ and Gwen C. Dieleman ${ }^{2}$

${ }^{1}$ Department of Complex Trait Genetics, Center for Neurogenomics and Cognitive Research, Vrije Universiteit Amsterdam, Amsterdam, The Netherlands

${ }^{2}$ Department of Child and Adolescent Psychiatry/Psychology, Erasmus University Medical Center, Rotterdam, The Netherlands

${ }^{3}$ Department of Clinical Genetics, Amsterdam UMC, Vrije Universiteit Medical Center, Amsterdam, The Netherlands

${ }^{4}$ Department of Psychiatry and Psychotherapy, Charite, Universitatsmedizin Berlin, Berlin, Germany

${ }^{5}$ Berlin School of Mind and Brain, Humboldt University of Berlin, Berlin, Germany

${ }^{6}$ Child and Adolescent Psychiatry and Psychosocial Care, Amsterdam UMC, Vrije Universiteit Amsterdam, Amsterdam Public Health, Amsterdam, The Netherlands

Keywords Genetics, Psychiatry, Neurodevelopmental disorders, Comorbidity, General P factor

Psychiatric traits are heritable, comorbid and genetically correlated making it plausible that there are shared genetic effects between these disorders. This study quantifies the predictive capacity of 14 polygenic risk scores (PRS) of traits related to psychiatric disorders by means of a case-control study in a child and adolescent sample $(\mathrm{N}=$ 1402) with mixed and multiple diagnoses and healthy controls $(\mathrm{N}=$ 1448). A PRS is a single measure of the common genetic risk an individual has for a certain trait. Included PRS are educational attainment, intelligence, smoking initiation, Neuroticism, insomnia, risk taking behavior, anti-social behavior, ADHD, ASD, schizophrenia, major depressive disorder, anxiety, alcohol dependence and bipolar disorder. These PRS were first individually tested in univariate regression model. A secondary analysis entails the testing of significantly associated PRS together in a multivariate model. The univariate analysis showed significant association with case control status for educational attainment, intelligence, smoking initiation, neuroticism, anti-social behavior, ADHD, major depressive disorder and alcohol dependence. The two main findings are educational attainment ( $\mathrm{p}$ value: $3.53 \mathrm{E}-20$, explained variance: $3.99 \%$, OR 0.66 ) and smoking initiation ( $\mathrm{p}$ value: $4.77 \mathrm{E}-10$, explained variance: $1.91 \%$, OR 1.33). The secondary analysis showed significant association of educational attainment and smoking initiation. The explained variance of the PRS in the multivariate model with these eight traits combined was $5.9 \%$. This study provides more insights into the genetic signal that is shared between childhood and adolescent psychiatric disorders might guide future studies on psychiatric comorbidity and offer insights into shared etiology between psychiatric disorders.

Grant Support: Sophia Stichting voor Wetenschappelijk Onderzoek (SSWO, grant number 593 and S14-27). 


\section{Systematic Identification of Genetic Loci Associated with Both Cannabis Use Disorder and Schizophrenia}

Emma C Johnson ${ }^{1}$, Sarah MC Colbert ${ }^{1}$, Alexander S Hatoum ${ }^{1}$, Joseph D Deak ${ }^{2,3}$, Renato Polimanti ${ }^{2,3}$, Robin Murray ${ }^{4}$, Howard J Edenberg ${ }^{5,6}$, Joel Gelernter ${ }^{2,3,7}$, Marta Di Forti ${ }^{8}$, Arpana Agrawal ${ }^{1}$

${ }^{1}$ Department of Psychiatry, Washington University School of Medicine, Saint Louis, Missouri, USA

${ }^{2}$ Department of Psychiatry, Yale University School of Medicine, New Haven, Connecticut, USA

${ }^{3}$ Department of Psychiatry, Veterans Affairs Connecticut Healthcare Center, West Haven, Connecticut, USA

${ }^{4}$ Department of Psychosis Studies, Institute of Psychiatry, Psychology and Neuroscience, King's College London, London, United Kingdom ${ }^{5}$ Department of Medical and Molecular Genetics, Indiana University School of Medicine, Indianapolis, Indiana, USA

${ }^{6}$ Department of Biochemistry and Molecular Biology, Indiana University School of Medicine, Indianapolis, Indiana, USA

${ }^{7}$ Departments of Genetics and Neuroscience, Yale University School of Medicine, New Haven, Connecticut, USA

${ }^{8}$ Social, Genetic and Developmental Psychiatry Centre, Institute of Psychiatry, Psychology and Neuroscience, King's College London, London, United Kingdom

Keywords Cannabis, Schizophrenia, Cross-disorder, Genome wide association study, Genetic overlap

Recent genome-wide association studies (GWAS) have found modest but significant genetic correlations between schizophrenia (SCZ) and cannabis ever-use (Pasman et al., 2018), and between SCZ and cannabis use disorder (CUD; Johnson et al., 2020). No study has yet examined the specific genetic loci and biological pathways associated with both CUD and SCZ liability. Using the largest genome-wide datasets available (Ns 46,213-632,802), we applied ASSET, a crossdisorder method, to identify $>100$ independent genome-wide significant loci pleiotropic for CUD and SCZ. A previously identified chromosome 8 locus that contains the genes EPHX2 and CHRNA2 showed a particularly strong signal for both CUD and SCZ, suggesting that this may be a point of shared genetic vulnerability. As tobacco smoking is phenotypically and genetically correlated with both CUD and SCZ, we also plan to use LAVA to examine bivariate and local genetic correlations between CUD and SCZ after conditioning on tobacco smoking and other potential confounds (e.g., SES).

\section{References}

Johnson, Emma C., et al. "A large-scale genome-wide association study meta-analysis of cannabis use disorder." The Lancet Psychiatry 7.12 (2020): 1032-1045.

Pasman, Joëlle A., et al. "GWAS of lifetime cannabis use reveals new risk loci, genetic overlap with psychiatric traits, and a causal effect of schizophrenia liability." Nature neuroscience 21.9 (2018): 1161-1170.

Grant Support: K01DA051759 (ECJ), DA054869 (AA, HJE, JG).
Harmonized Phenotypes for Anxiety, Depression, and Attention-Deficit Hyperactivity Disorder (ADHD)

Miljan Jović ${ }^{1, *}$, Kratika Agarwal ${ }^{1, *}$, Andrew Whitehouse ${ }^{2}$, Stéphanie M. van den Berg ${ }^{1}$

*Shared first authorship

${ }^{1}$ Department of Learning, Data Analytics and Technology, University of Twente, Enschede, The Netherlands

${ }^{2}$ Telethon Kids Institute, University of Western Australia, Perth, Australia

Keywords Data harmonization, Anxiety, Depression, ADHD, Raine study

In multi-cohort consortia, the problem often arises that a phenotype is measured using different questionnaires in different cohorts. This study aimed to harmonize scores based on the Child Behaviour Check List (CBCL) and the Strength and Difficulties Questionnaire (SDQ) for anxiety/depression and ADHD using test linking. To link the scales, we used parent reports on 1330 children aged 10-11.5 years from the Raine study on both SDQ and CBCL. Test linking was done based on Item Response Theory. We started from existing CBCL and SDQ scales related to anxiety/depression and ADHD (theoretical approach). For confirmation, we conducted a data-driven approach using factor analysis to validate the theoretical approach. Both approaches yielded similar scales, supporting the combination of existing scales. In addition, we studied the impact of harmonizing scores on the statistical power of meta-analytic gene-finding studies. The results showed that IRT-based harmonization increased the statistical power of the results compared to sum scores, even with equal sample size. These findings can guide future researchers to harmonize data from different samples and/or different questionnaires that measure anxiety, depression, and ADHD, in order to obtain larger sample sizes, to compare research results across subpopulations, or to increase generalizability of research results. We recommend using our item parameters to estimate harmonized scores that represent commensurate phenotypes across cohorts, and we explained in detail how other researchers can use our results to harmonize data in their studies.

Grant Support: This work has been supported by the CAPICE project, funded by the European Union's Horizon 2020 Research and Innovation Programme under the Marie Sklodowska-Curie grant agreement no. 721567 . We are grateful to the Raine Study participants and their families and we thank the Raine Study team for cohort co-ordination and data collection. The core management of the Raine Study is funded by The University of Western Australia, Curtin University, Telethon Kids Institute, Women and Infants Research Foundation, Edith Cowan University, Murdoch University, The University of Notre Dame Australia, and the Raine Medical Research Foundation. AJOW is supported by an Investigator Grant from the National Health and Medical Research Council (APP1173896). 


\section{A Role of Adolescent Lifestyle Habits in Biological Aging: A Prospective Twin Study}

\author{
Anna Kankaanpää ${ }^{1}$, Asko Tolvanen ${ }^{2}$, Aino Heikkinen ${ }^{3}$, \\ Jaakko Kaprio $^{3}$, Miina Ollikainen ${ }^{3,4}$, Elina Sillanpää ${ }^{1,3}$ \\ ${ }^{1}$ Gerontology Research Center (GEREC), Faculty of Sport and Health \\ Sciences, University of Jyväskylä, Jyväskylä, Finland \\ ${ }^{2}$ Methodology Center for Human Sciences, University of Jyväskylä, \\ Jyväskylä, Finland \\ ${ }^{3}$ Institute for Molecular Medicine Finland (FIMM), HiLife, Univer- \\ sity of Helsinki, Helsinki, Finland \\ ${ }^{4}$ Department of Public Health, University of Helsinki, Helsinki, \\ Finland
}

Keywords Twin study, Epigenetic clock, Biological aging, Lifestyle, Adolescence, Latent class analysis

Adolescence is a stage of fast growth and development. Exposures during puberty may have long-term effects on health in later life. In this study, we aimed to investigate the role of adolescent lifestyle in biological aging. Biological aging was assessed in young adulthood by four measures of epigenetic aging. Study participants were twin pairs $(\mathrm{n}=371)$ aged $21-25$ years from the Finnish Twin Cohort. Blood-based DNA methylation (DNAm) was used to assess epigenetic aging by Horvath's clock, DNAm PhenoAge, DNAm GrimAge, and DunedinPoAm estimators. Adolescent lifestyle-related factors, including body mass index, leisure-time physical activity, smoking, and alcohol use were measured at ages of 12, 14, and 17 years. First, latent class analysis was conducted to identify patterns of lifestyle behaviors in adolescence. We identified six subgroups of participants with different lifestyle behavior patterns. Second, the mean differences across the subgroups in later biological aging were studied using the Bolck-Croon-Hagenaars $(\mathrm{BCH})$ approach. The groups with the healthier lifestyle behaviors were biologically younger compared to the groups with unhealthy habits. However, the differences were observed only when DNAm GrimAge and DunedinPoAm estimators were used to assess biological aging. These findings suggest that adolescent lifestyle may associate with biological aging process. Of measures of epigenetic aging, DNAm GrimAge and DunedinPoAm estimators may capture adolescent lifestyle-induced changes in biological aging. We are currently extending our analysis to include ACE components. Our purpose is to investigate the genetic and environmental factors underlying the observed differences in biological aging between the subgroups.

Grant Support: This work was supported by the Academy of Finland [213506, 265240, 263278, 312073 to JK, and 297908 to MO], EC FP5 GenomEUtwin (JK), NIH NIH/NHLBI (grant HL104125), EC MC ITN Project EPITRAIN (JK \& MO) project and the University of Helsinki Research Funds to MO, Sigrid Juselius Foundation to JK and MO, Yrjö Jahnsson foundation (6868) and Juho Vainio foundation to ES.

\section{Large-Scale Cross-Disorder Study on Penetrance and Comorbidity of Neuropsychiatric Copy-Number Variants}

Richard Karlsson Linnér*1,2, Matthew T Oetjens* ${ }^{*}$, Juliette Gudknecht ${ }^{1}$, Christopher F. Chabris ${ }^{1}$, Scott M. Myers ${ }^{1}$, Brenda Finucane ${ }^{1}$, David H. Ledbetter ${ }^{1}$, Christa L. Martin ${ }^{1}$
${ }^{1}$ Autism and Developmental Medicine Institute (ADMI), Geisinger, Lewisburg, Pennsylvania, USA

${ }^{2}$ School of Business and Economics, Vrije Universiteit Amsterdam, Netherlands

*Correspondence to: rklinner@geisinger.edu mtoetjens@geisinger.edu

Keywords Polygenic Risk Scores, Copy-Number Variants, Neurodevelopmental and Psychiatric Disorders, Cross-Disorder Study

Rare copy-number variants (CNVs) greatly increase the risk of neuropsychiatric disorders, but clinical interpretation and genetic counselling remains challenging because of incomplete penetrance and variable expressivity. Recent studies suggest that polygenic scores can help, but few studies have simultaneously investigated common and rare variation in samples not ascertained for a specific disorder or variant. Here, in a large exome-sequenced sample of MyCode 'DiscovEHR' participants $(N=122,370)$, we study the joint contribution of 26 rare $\mathrm{CNVs}\left(N_{\mathrm{CNV}+}=933\right)$, e.g., $22 \mathrm{q} 11.2$ deletion (DiGeorge) syndrome $(N=21)$, and six polygenic scores, to the risk of five disorders: attention-deficit/hyperactivity disorder (ADHD; 3779 cases), autism spectrum disorder (ASD; 450 cases), bipolar disorder (BD; 6678 cases), intellectual disability (ID; 676 cases), and schizophrenia (SCZ; 920 cases). Our findings replicate known CNVdisorder associations and provide new "non-ascertained" estimates, in particular for ADHD. Cross-disorder polygenic score analysis generally improved model fit, except for ID. Although the effects of the polygenic scores on the five disorders could be considered small, the scores jointly explained more variation in ADHD, BD, and SCZ than the CNVs combined. We find general support for an additive model where people with rare $\mathrm{CNV}$ s require less polygenic burden to develop disorder. We conclude that polygenic scores should indeed be able to improve clinical interpretation of rare CNVs, but that adequately powered replication within this high-risk group requires a seven times larger sample of $\mathrm{CNV}+$ participants.

Grant Support: NIH 5U01MH119705-03 Marin \& Ledbetter.

\section{APOE-Controlled Effect of Inflammatory System Gene Variants on Non-verbal Intelligence in Young Adults}

Anastasiya Kazantseva ${ }^{1,2}$, Renata Enikeeva ${ }^{1,2}$, Yuliya Davydova ${ }^{1}$, Zalina Takhirova $^{2}$, Rustam Mustafin ${ }^{3}$, Marina Lobaskova ${ }^{4}$,

Yuliya Galyautdinova ${ }^{5}$, Sergey Malykh ${ }^{4,6}$, Elza Khusnutdinova ${ }^{1,6}$

${ }^{1}$ Department of Human Molecular Genetics, Institute of Biochemistry and Genetics - Subdivision of the Ufa Federal Research Centre of the Russian Academy of Sciences, Ufa, Russian Federation

${ }^{2}$ Centre for interdisciplinary research in the field of educational sciences, Russian Academy of Education, Moscow, Russian Federation ${ }^{3}$ Department of Medical Genetics and Fundamental Medicine, Bashkir State Medical University, Ufa, Russian Federation

${ }^{4}$ Laboratory of age psychogenetics, Psychological Institute of the Russian Academy of Education, Moscow, Russian Federation

${ }^{5}$ Department of Genetics and Fundamental Medicine, Bashkir State University, Ufa, Russian Federation

${ }^{6}$ Faculty of psychology, Lomonosov Moscow State University, Moscow, Russian Federation.

Keywords Cognitive ability, g-factor, APOE, Tumor necrosis factor, Haplotype

Although multiple studies indicated the role of APOE gene in cognitive decline, the evidence of APOE effect on genetic associations of inflammatory system genes and cognitive abilities (including non- 
verbal intelligence, NVI) is scarce. The aim of the study was to estimate the main effect of inflammatory gene variants on individual differences in non-verbal intelligence in mentally healthy students and to clarify the necessity to control for unfavorable APOE E4alleles while performing genetic testing even at young age. The study included 1011 mentally healthy individuals (80\% women; $19.79 \pm$ 1.69 years) of Caucasian origin (535 Russians, 231 Tatars, 160 Udmurts, and 85 of mixed ethnicity) from Russia. NVI score was assessed via Raven's progressive matrices. The IL1b rs16944, IL1A rs1800587, CRP rs3093077, TNF rs1041981 and rs1800629, $P 2 X 7 R$ rs2230912 gene variants were genotyped using PCR. Statistical analysis included multiple linear regression models (additive, dominant, recessive) conducted controlling for sex, ethnicity and $A P O E$ E4-allele in total sample and in men and women separately (PLINK v.1.09). While stratifying by $A P O E$ E4-allele and controlling for sex and ethnicity in the total sample, there was a significant effect of TNF rs1800629 A-allele $(\beta=1.79 ; \mathrm{P}=0.019), T N F$ rs1041981 A-allele $(\beta$ $=1.49 ; \mathrm{P}=0.019)$ and TNF AA-haplotype (rs1041981, rs1800629) $(\beta$ $=1.53 ; \mathrm{P}=0.033$ ) on higher NVI in dominant model. The same effect was observed in men while controlling for APOE E4-allele: TNF rs1800629 A-allele was associated with higher NVI $(\beta=1.75 ; \mathrm{P}=$ $0.021)$. The findings obtained evidence in a modulating effect of $A P O E$ E4-allele on the association between TNF gene variants and non-verbal intelligence in young adults.

Grant Support: The present study was supported by the Russian Science Foundation (project no. 17-78-30028).

\section{Predicting School Performance by Non-cognitive Factors Self-control and Grit in a Genetically Informed Design}

Sofieke T. Kevenaar ${ }^{1}$, Conor V. Dolan ${ }^{1}$, Eveline L. de Zeeuw ${ }^{1}$, Dorret I. Boomsma ${ }^{1}$, Elsje van Bergen ${ }^{1}$

${ }^{1}$ Netherlands Twin Register, Department of Biological Psychology, Vrije Universiteit Amsterdam, Amsterdam, The Netherlands

Keywords Self-control, Grit, School performance, SES, Genetically Informed Regression Analysis

Understanding individual differences in school performance has been a topic of interest for decades. Non-cognitive factors have gained increasing attention as a possible explanation on why some children do better in school than others. Two non-cognitive factors that have been related to school success are self-control and grit. We analyzed teacher-rated data that indexed these non-cognitive skills (self-control and grit) and data on academic performance in twelve-year-old twins from the Netherlands Twin Register $(\mathrm{N} \leq 9568)$. Information on parental SES was available from parental reports on educational attainment and occupation. Children from high SES had better academic performance, more self-control and were grittier than children from lower SES backgrounds. Girls had better academic performance, more self-control and were grittier than boys. We tested for moderation of genetic and environmental variance components by SES and saw no evidence for SES by genotype or SES by environment interaction. However, the variance in children from higher SES backgrounds was lower. This indicates that children from higher SES backgrounds are more similar to each other than lower SES children, but the portion of these individual differences that can be explained by genetic and environmental factors is the same across SES strata. Next, we will test the differential prediction of self-control and grit for school performance, by simultaneously fitting a genetic covariance structure model and a regression model to these data from mono- and dizygotic twins. This method allows for differential prediction of school performance by the correlated non-cognitive factors self-control and grit.

Grant Support: Gravitation program of the Dutch Ministry of Education, Culture, and Science and the Netherlands Organization for Scientific Research (NWO grant number 024.001.003).

\section{Increasing Heritability of Late-Life Cognition: The Case for Phenotype-Environment Effects}

Alice J. Kim, MA ${ }^{1}$, Evan J. Giangrande, $\mathrm{MA}^{2}$, and Christopher R. Beam, $\mathrm{PhD}^{1}$

${ }^{1}$ Department of Psychology, University of Southern California, Los Angeles, CA, USA

${ }^{2}$ Department of Psychology, University of Virginia, Charlottesville, VA, USA

Keywords Heritability, Older adults, Cognition, Phenotype-environment

Heritability of cognitive ability increases over time and accounts for the majority of variance in cognitive aging outcomes. The processes that lead to differentiation within aging families remains unclear. We hypothesized that the accumulation of within-family gene-environment correlation would cause dizygotic twins to decrease in similarity with age while monozygotic twin similarity would remain constant with age. We analyzed six waves of data from 3565 same-sex twin families ( $\geq 70$ years; $58.7 \%$ women; $N=4457$ ) collected every two years between 1995 and 2005 in the Longitudinal Study of Aging Danish Twins $(\mathrm{MZ}=1576 ; \mathrm{DZ}=2881)$. At each wave, global cognitive functioning was assessed. Multilevel SEM was used to test whether family gene-environment correlation increases in DZ twins to cause heritability to increase with age. Results indicate that MZ twin correlations increased over time, increasing from .51 to .69. DZ twin correlations stayed stable over ten years, increasing from .31 to .38. Model fitting results suggest that between-family gene-environment correlative processes, not within-family gene-environment correlation, are implicated in the increasing heritability of late-life cognition. Study findings suggest that between-family gene-environment correlation may have an important role in how heritability of cognition increases with age.

Grant Support: N/A.

\section{Determine the Assortative Mating Pattern of the Phenotypes by Using Covariance Structures of the Haplotypic Polygenic Scores}

Yongkang Kim ${ }^{1}$, Jared V Balbona ${ }^{1,2}$, Emmanuel Sapin ${ }^{1}$, and Matthew C Keller ${ }^{1,2}$

${ }^{1}$ Institute for Behavioral Genetics, University of Colorado at Boulder, Boulder, Colorado, USA

${ }^{2}$ Department of Psychology, University of Colorado at Boulder, Boulder, Colorado, USA

Keywords Genome-wide association studies (GWAS), Polygenic risk score, Assortative mating, Structural equation modeling (SEM), Goodness-of-fit test 
Assortative mating (AM) can bias estimates of genetic effects from family/twin-based and genomics-based approaches. Properly adjusting estimates requires knowing whether the $\mathrm{AM}$ is at equilibrium or not. Here, we show how different types of AM (at equilibrium vs. AM for one or two previous generations) lead to different patterns of 6 unique covariances between the haplotypic polygenic scores (hPGS's) of spouses. For example, all covariances are expected to be zero between hPGSs of spouses when there is no AM, all 6 are expected to be positive and equal under equilibrium AM, and 4 of the 6 are expected to be positive under a single generation of AM. In addition, the 4 variances of the hPGS are similarly informative. We show how a goodness of fit test on these hPGS variance/covariances matrices (e.g., using openMx) can be used to test whether the data best fits a single generation of AM model vs. an equilibrium AM model. We show further that spousal data that is "gametically phased" (such that the chromosomes inherited from different parents are differentiated) can be used to test for AM that has occurred for one generation vs. two generations vs. many generations.

Grant Support: MH100141 (Keller).

\section{Intergenerational Transmission of ADHD: More Evidence for Heritability than Fast Life History}

Thomas Haarklau Kleppest $\varnothing^{b^{*}}$, Espen Moen Eilertsen ${ }^{\mathrm{a}, \mathrm{b}, \mathrm{c}}$, Elsje van Bergen ${ }^{\text {e,f,g }}$, Brendan Zietsch ${ }^{\mathrm{i}}$, Hans Fredrik Sunde ${ }^{\mathrm{b}}$, Magnus Nordmo ${ }^{\mathrm{b}}$, Nikolai Haahjem Eftedal ${ }^{\mathrm{a}, \mathrm{c}}$, Alexandra Havdahl ${ }^{\mathrm{b}, \mathrm{h}, \mathrm{c}}$, Eivind Ystrom ${ }^{\mathrm{a}, \mathrm{b}, \mathrm{d}}$ * , Fartein Ask Torvik ${ }^{\mathrm{a}, \mathrm{b}}$ * ${ }^{a}$ Department of Psychology, University of Oslo, Norway

${ }^{\mathrm{b}}$ Norwegian Institute of Public Health, Oslo, Norway

${ }^{\mathrm{c}}$ PROMENTA Research Center, Department of Psychology, University of Oslo, Norway

${ }^{\mathrm{d}}$ School of Pharmacy, University of Oslo, Norway

${ }^{\mathrm{e}}$ Department of Biological Psychology, Vrije Universiteit Amsterdam, The Netherlands

${ }^{\mathrm{f}}$ Research Institute LEARN!, Vrije Universiteit Amsterdam, The Netherlands

${ }^{\mathrm{g}}$ Amsterdam Public Health research institute

${ }^{\mathrm{h}}$ Nic Waals Institute, Spångbergveien 25, 0853 Oslo, Norway

${ }^{\mathrm{i}}$ Centre for Psychology and Evolution, School of Psychology, University of Queensland, St. Lucia, Brisbane, QLD 4072, Australia.

\section{Keywords ADHD, Lifehistory, Evolution, Children-of-twins}

Parents and children resemble each other in their levels of ADHD symptoms. Twin studies suggest that ADHD is heritable, but also that environmental factors play a role. A key theoretical postulate from life history theory (LHT) is that children use information from their environment (e.g., predictability and resource availability) and develop phenotypes that are optimized for future success in that environment. A key source of environmental information are their parents. From an LHT perspective, it is therefore expected that variation in children's ADHD symptoms reflect the information they have gathered from parental behavior and the larger developmental context the parents provide. The alternative hypothesis is that parents and children resemble each other because of transmitted genes. This study uses a large children-of-twins sample $(\mathrm{N}=22,350$ parents; 11,566 children) which allows us to disentangle genetic transmission from environmental transmission (i.e., effects of parental behavior). The effect of parents on children was largely due to genetic transmission. Children's ADHD levels were largely driven by heritability $(57 \%)$, with small effects of parental environment (2\%), gene-environment correlation $(2 \%)$, and the remainder due to unique environmental influences and noise. We conclude that ADHD is not the outcome of adaptive behavior to the (family) environment, but rather a neurodevelopmental disorder with a strong genetic basis.

\section{Genome-Wide Association Study of Liking of Physical Activity in the UK Biobank}

Yann C. Klimentidis ${ }^{1}$, Michelle Newell ${ }^{1}$, Matthijs D. van der Zee ${ }^{2,3}$, Victoria L. Bland ${ }^{4}$, Sebastian May-Wilson ${ }^{5}$, Amit Arora ${ }^{1}$, David A. Raichlen ${ }^{6}$, Gene E. Alexander ${ }^{7}$, Jouke-Jan Hottenga ${ }^{2,3}$, Eco J.C. de Geus ${ }^{2,3}$, Nicola Pirastu ${ }^{5}$

${ }^{1}$ Department of Epidemiology and Biostatistics, Mel and Enid Zuckerman College of Public Health, University of Arizona, Tucson, AZ, USA

${ }^{2}$ Department of Biological Psychology, Vrije Universiteit Amsterdam, The Netherlands

${ }^{3}$ Netherlands Twin Register, The Netherlands

${ }^{4}$ Department of Nutritional Sciences, University of Arizona, Tucson, AZ, USA

${ }^{5}$ Centre for Global Health Research, Usher Institute for Population Health Sciences and Informatics, University of Edinburgh, Edinburgh, United Kingdom

${ }^{6}$ Human and Evolutionary Biology Section, Department of Biological Sciences, University of Southern California

${ }^{7}$ Department of Psychology, University of Arizona, Tucson, AZ, USA

Keywords Physical activity, Exercise, Liking, Preference, Genetic

A lack of physical activity (PA) is one of the most pressing current health issues. In addition to economic and social influences, one's propensity for PA is influenced by genetic factors. Here, in over 157,000 individuals from the UK Biobank, we sought to complement and extend previous findings on the genetics of PA behavior by performing genome-wide association studies of liking of several PArelated behaviors plus an additional derived overall PA-liking trait. We identified a total of 17 loci, along with an additional 8 for the overall trait, only some of which overlap with loci previously identified for PA behavior. Replication in over 7000 adults from the Netherlands Twin Register (NTR) showed directional consistency in 13 out of 17 loci. The PA-liking traits were genetically correlated with self-report $\left(r_{\mathrm{g}}: 0.38-0.80\right)$ and accelerometry-derived $\left(\mathrm{r}_{\mathrm{g}}\right.$ : 0.26-0.49) PA measures, and with a wide range of health-related traits and dietary behaviors. Polygenic risk scores (PRS) for each PAliking trait computed in NTR based on UKB significantly predicted the same liking trait in NTR. The PRS for overall PA-liking predicts PA behavior in NTR $\left(r^{2}=0.0032\right)$ nearly as well as one constructed based on PA behavior in UK Biobank $\left(r^{2}=0.0042\right)$. Combining the two PRS in a single model increased $r^{2}$ to 0.0049 , suggesting that although they broadly overlap, they are capturing different dimensions of PA behavior. In conclusion, we have identified some of the first loci associated with PA-liking and extend our understanding of the genetic basis of PA behavior. 


\section{First Results from a Multivariate GWAS on Different Measures of Income Among $\approx \mathbf{7 5 6 , 0 0 0}$ Individuals}

Casper A.P. Burik ${ }^{1}$, H. Kweon ${ }^{1}$, A. Okbay ${ }^{1}$, R. Karlsson Linnér ${ }^{1}$, R. de Vlaming ${ }^{1}$, Daniel J. Benjamin ${ }^{2,3,4}$, Thomas A. DiPrete ${ }^{5}$, Philipp D. Koellinger ${ }^{1,6}$, the Social Science Genetic Association Consortium (SSGAC)

${ }^{1}$ Department of Economics, School of Business and Economics, Vrije Universiteit Amsterdam, Amsterdam, The Netherlands

${ }^{2}$ National Bureau of Economic Research, Cambridge, MA, USA

${ }^{3}$ UCLA Anderson School of Management, Los Angeles, CA, USA

${ }^{4}$ Human Genetics Department, UCLA David Geffen School of Medicine, Los Angeles, CA, USA

${ }^{5}$ Department of Sociology, Columbia University, New York, New York, USA

${ }^{6}$ La Follette School of Public Affairs, University of WisconsinMadison, Madison, Wisconsin, USA

\section{Keywords GWAS, Income, Meta-analysis}

Poverty and economic deprivation are known to be major risk factors for mental and physical diseases as well as for lower life expectancy. Thus, a precise understanding of how income inequalities are related to health inequalities is of fundamental importance for science, social policy, and public health. In 2018, the Social Science Genetic Association Consortium (SSGAC) launched a genome-wide association study (GWAS) meta-analysis on income with the purpose to generate well-powered, publicly available GWAS summary statistics that will provide researchers from various disciplines with new ways to study the causes and consequences of inequalities in wealth and health. Thirty one cohorts from Europe, the US, and Australia joined the project and provided GWAS results on personal income, household income, occupational wages, or parental income from $\approx 756,000$ individuals of European ancestries. The SNP-based narrow-sense heritability of these income measures varies between 4 and 15 percent, partly driven by differences in measurement accuracy. After rigorous quality control, a multi-trait analysis of GWAS summary statistics (MTAG) of all four income measures identified 178 independent genetic loci. The genetic correlations of income across cohorts and between men and women are imperfect $(<1)$. Our results are consistent with the hypothesis that substantial differences in environmental conditions influence labor market outcomes. Furthermore, we find that income is genetically correlated with educational attainment $(\sim 0.9)$, cognitive performance $(\sim 0.6)$, and a broad variety of health outcomes across different body parts and organs.

Grant Support: The study was financially supported by a variety of research grants, including a European Research Council Consolidator Grant (647648 EdGe to Koellinger), a Netherlands Organisation for Scientific Research VENI grant (NWO 016.Veni.198.058 to Okbay), and a Riksbankens Jubileumsfond grant (P18-0782:1).

\section{Association Between Combinations of Genotypes of Genes MAOA, COMT, 5HTR2A, DRD4 with Emotional and Personal Characteristics of Young People, Living in the South of Russia}

Ekaterina M Kovsh ${ }^{1}$, Elena V Vorobyeva ${ }^{1,2}$, Ekaterina G Denisova ${ }^{2}$, Daria S Alekseeva ${ }^{1}$, Vitaly V Babenko ${ }^{1,2}$, Pavel N Ermakov ${ }^{1}$.

${ }^{1}$ Academy of Psychology and Educational Sciences, Southern Federal University, Rostov-on-Don, Russian Federation

${ }^{2}$ Faculty "Psychology, pedagogy and defectology", Don State Technical University, Rostov-on-Don, Russian Federation

Keywords MAOA, COMT, 5HTR2A, DRD4, Emotional intelligence

Introduction. The association of combinations of neurotransmitter systems genes' genotypes (MAOA, COMT, 5HTR2A, DRD4) with peculiarities of the emotional and personal sphere (the level of empathy, emotional intelligence-EmIn, alexithymia) were studied on a sample of 100 Russian right-handed psychology students without health problems (average age-19.3; 68\%-women). Genetic methods: DNA analysis, PCR, agarose gel electrophoresis ("Biological Solutions and Technologies", Russia). Psychodiagnostics (questionnaires): 5PFQ, TAS-26, EETS, The test of EmIn by D.V. Lyusin. Statistics: ANOVA (Statistica 13.0), Tukey's post-hoc test for nonequilibrium sample sizes. Tukey post-hoc test (ANOVA) revealed the following $(\mathrm{p}<0.05)$ : combinations of highly active MAOA $(\mathrm{H})$ and $\mathrm{Val} / \mathrm{Val}$ COMT genotypes $(\mathrm{M}=95.3)$ and low-level MAOA $(\mathrm{L})$ and Met/Met COMT $(M=91.6)$ genotypes are associated with a high level of general EmIn; the combination of heterozygous genotypes of the MAOA (M) and Val/Met COMT genes is associated with a significantly lower level of EmIn $(M=95.3)$; carriers of the combination of the heterozygous genotype of the MAOA (M) gene and the Met/ Met COMT genotype have a significantly higher level of empathy in the study sample $(M=28.5)$; men who carry a combination of Met/ Met COMT and T/T DRD4 $(M=9)$ genotypes, as well as carriers of a combination of T/T 5HTR2A and a highly active genotype of the gene MAOA $(M=13)$, have a significantly lower level of empathy in the studied sample. Combinations of genes of neurotransmitter systems are associated with the level of empathy and EmIn, but not with the level of alexithymia.

Grant Support: This study was supported by the Russian Science Foundation (RSF), research project No. 20-64-47057. 


\section{Socioeconomic Status and Its Genetic Basis are Embodied in Human Brain Anatomy}

Hyeokmoon Kweon ${ }^{1}$, Gökhan Aydogan ${ }^{2}$, Alain Dagher ${ }^{3}$, Danilo Bzdok ${ }^{3,4,5,6}$, Gideon Nave ${ }^{7}$, Martha J. Farah ${ }^{8}$,

Philipp Koellinger ${ }^{1,9}$

${ }^{1}$ Department of Economics, School of Business and Economics, Vrije Universiteit Amsterdam, $1081 \mathrm{HV}$ Amsterdam, Netherlands

${ }^{2}$ Zürich Center for Neuroeconomics (ZNE), Department of Economics, University of Zurich, 8006 Zürich, Switzerland

${ }^{3}$ McConnell Brain Imaging Centre, Montreal Neurological Institute (MNI), McGill University, Montreal, QC H3A 2B4, Canada

${ }^{4}$ Department of Biomedical Engineering, Faculty of Medicine, McGill University, Montreal, QC H3A 2B4, Canada

${ }^{5}$ School of Computer Science, McGill University, Montreal, QC H3A 2A7, Canada

${ }^{6}$ Mila - Quebec Artificial Intelligence Institute, Montreal, QC H2S 3H1, Canada

${ }^{7}$ Marketing Department, the Wharton School, University of Pennsylvania, Philadelphia, PA 19104, USA

${ }^{8}$ Center for Neuroscience \& Society, University of Pennsylvania, Philadelphia, PA 19104, USA

${ }^{9}$ La Follette School of Public Affairs, University of WisconsinMadison, Madison, WI 53706, USA

Keywords Socioeconomic status, Polygenic score, Brain, Voxel-based morphometry, Grey matter volume

The brain is the major locus of integration for genetic and environmental influences on behavior, including those related to socioeconomic status (SES). Current insights into the relationships between brain anatomy and SES are limited. Furthermore, little is known about whether such relationships are due to environmental or genetic effects. To address these questions, we conduct a genetically informed neuroscientific study on SES in a population sample of 23,714 adults from the UK. We find many small, positive associations (partial $R^{2}<1.3 \%$ ) between SES and voxel-level grey matter volume (GMV) across the entire brain. A brainwide GMV index predicts $5 \%$ of the variance in SES out-of-sample. We show that SES and GMV structure have a partially shared common genetic architecture, in particular in regions of the insular and prefrontal cortex. Controlling for the genetic underpinnings of SES reduces the associations between SES and GMV on average by 38\%. SES-GMV associations are most robust to genetic controls in the cerebellar, lateral-temporal, and lateral-parietal regions, suggesting that these regions are more prone to environmental influences. Furthermore, we find that SESGMV associations can be partially attributed to individual differences in body mass index even after adjusting for genetic controls. This suggests that environmental factors that lead to obesity among low SES individuals also have negative consequences for brain health. Thus, the patterns we observe are both anatomical reflections of persisting socio-economic inequalities and a window into how unfavorable environments pose a risk to brain health and social mobility.

Grant Support: The study was supported by funding from an ERC Consolidator Grant to PK (647648 EdGe), an NSF Early Career Development Program grant (1942917) to GN, and the Wisconsin Alumni Research Foundation, provided to PK by the Office of the Vice Chancellor for Research and Graduate Education at the University of Wisconsin-Madison. GN thanks Carlos and Rosa de la Cruz for ongoing support.

\section{Longitudinal Change in Heritability of Verbal Episodic Memory: Preliminary Findings from the Older Australian Twins Study (OATS)}

Teresa Lee ${ }^{1,2}$, Anbupalam Thalamuthu ${ }^{1}$, Ben Lam ${ }^{1}$, Dansen Cho ${ }^{1}$, Julie D Henry ${ }^{3}$, Margaret J Wright ${ }^{4}$, Julian N Trollor ${ }^{1,5}$, Vibeke S Catts ${ }^{1}$, Perminder Sachdev ${ }^{1,2}$

${ }^{1}$ Centre for Healthy Brain Ageing, School of Psychiatry, University of New South Wales, Sydney, NSW 2052, Australia

${ }^{2}$ Neuropsychiatric Institute, Euroa Centre, Prince of Wales Hospital, Randwick, NSW, Australia

${ }^{3}$ School of Psychology, University of Queensland, Brisbane, QLD 4072, Australia

${ }^{4}$ Queensland Brain Institute, University of Queensland, Brisbane, QLD 4072, Australia

${ }^{5}$ Department of Developmental Disability Neuropsychiatry, School of Psychiatry, University of New South Wales, Sydney, NSW 2052, Australia

Keywords Heritability, Longitudinal, Episodic memory, Aging

Episodic memory (EM) decline/impairment is a salient feature of cognitive aging and neurodegenerative conditions, such as amnestic Mild Cognitive Impairment and Alzheimer's disease. While longitudinal EM changes in cognitive aging have been frequently studied, longitudinal change in the heritability of EM has not been fully explored. We used a subsample of $148 \mathrm{MZ}$ and $116 \mathrm{DZ}$ twin pairs (mean age 71 at baseline, 65\% female) from OATS to examine change in heritability of four measures of EM (immediate story recall, delayed story recall, word-list total learning over repetitions, and delayed word-list recall) at baseline and at a mean follow-up assessment of 5.3 years. Residuals of EM measures were obtained after adjusting for age, sex, education, mood (Geriatric Depression Scale), global cognition and practice effects. A saturated Cholesky ACE model was fitted using the residuals. Preliminary results suggested moderate increase in heritability for immediate story recall $(0.35,95 \% \mathrm{CI}[0.25,0.44])$ and delayed story recall $(0.40[0.30,0.49]$ at baseline to follow-up $(0.52[0.41,0.63]$ and $0.48[0.37,60]$, respectively). A decreased trend was observed for word-list total learning $(0.41[0.28,0.49])$ and delayed word-list recall $(0.44$ $[0.31,0.54])$ at baseline to follow-up $(0.37[0.19,0.50]$ and 0.37 [0.24,0.47], respectively). However, overlapping confidence intervals suggest that changes in heritability may not be statistically significant. Latent change model will be fitted to examine the degree of change in EM between two time-points, to assess change in heritability, and to assess if the change is associated with the general EM domain or test specific.

Grant Support: We acknowledge the contribution of the OATS research team (https://cheba.unsw.edu.au/project/older-australiantwins-study) to this study. The OATS study has been funded by a National Health \& Medical Research Council (NHMRC) and Australian Research Council (ARC) Strategic Award Grant of the Ageing Well, Ageing Productively Program (ID No. 401162); NHMRC Project (seed) Grants (ID No. 1024224 and 1025243); NHMRC Project Grants (ID No. 1045325 and 1085606); and NHMRC Program Grants (ID No. 568969 and 1093083). We thank the participants for their time and generosity in contributing to this research. This research was facilitated through access to Twins Research Australia, a national resource supported by a Centre of Research Excellence Grant (ID No. 1079102) from the National Health and Medical Research Council. 


\section{Breaking the Link Between Genetic Risk and Externalizing Problems Through Early Prevention}

\author{
Kathryn Lemery-Chalfant ${ }^{1}$, Veronica Oro ${ }^{1}$, Sierra Clifford ${ }^{1}$, \\ Melvin Wilson ${ }^{2}, \&$ Daniel Shaw ${ }^{2}$ \\ ${ }^{1}$ Department of Psychology, Arizona State University, Tempe, Ari- \\ zona, USA \\ ${ }^{2}$ Department of Psychology, University of Virginia, Charlottesville, \\ Virginia, USA \\ ${ }^{3}$ Department of Psychology, University of Pittsburgh, Pittsburgh, \\ Pennsylvania, USA
}

Keywords Prevention, Polygenic, Aggression, Alcohol, Developmental

Because genes and environments work together to influence health and development (i.e., the nature-nurture debate is dead), psychosocial interventions should attenuate genetic risk. The Family Check-Up (FCU) has been shown to increase children's effortful control, and reduce psychopathology and substance use. We hypothesized that FCU would mitigate genetic risk for aggression and frequency of alcohol use by increasing children's effortful control. The Early Steps RCT included 731 racially/ethnically diverse low-income children (49\% females; $M=29.9$ months at baseline) and their parents. Based on recent GWAS, polygenic risk scores for childhood aggression and adult alcohol use were formed. Children's effortful control was assessed at home visits, and parent-reported effortful control was obtained across middle childhood. Covariates included genetic ancestry PCs, age, sex, and socioeconomic status. Using an intent to treat design, FCU moderated the association between aggression polygenic risk and effortful control, such that polygenic risk was associated with lower effortful control in the control group (simple slope: $B=-.263, \mathrm{SE}=.053, p<.001$ ) but not the intervention group (simple slope: $B=-.069, \mathrm{SE}=.051, n s$ ). A similar pattern was found with genetic risk for alcohol use. Children at higher genetic risk for aggression and frequency of alcohol use had lower levels of effortful control. Importantly, FCU moderated both types of genetic risk by "repairing" children's "too low" effortful control. Findings support contemporary theories of gene-environment interplay, underscoring the importance of early prevention for mitigating both genetic and environmental risk of externalizing problems.

Grant Support: R01 DA036832

\section{Investigating the Etiology of Self-harm by Integrating Mendelian Randomization within Twin Modelling}

Kai Xiang Lim $^{1}$, Olakunle Oggini ${ }^{1}$, Kaili Rimfeld ${ }^{1}$, Jean-Baptiste Pingault ${ }^{1,2}$, Fruhling Rijsdijk ${ }^{1}$.

${ }^{1}$ Social, Genetic and Developmental Psychiatry Centre, Institute of Psychiatry, Psychology and Neuroscience, King's College London, London, UK.

${ }^{2}$ Department of Clinical, Educational and Health Psychology, Division of Psychology and Language Sciences, University College London, London, UK

Keywords Self-harm, Mendelian randomization, Direction of causation, Suicide attempt, Non-suicidal self-harm

Self-harm can be further delineated into suicidal self-harm (SSH) and non-suicidal self-harm (NSSH). By fitting Mendelian Randomization - Direction of Causation (MR-DoC) models, we investigated if there is any etiological difference between NSSH and SSH in terms of their causal relationships with mental health conditions. We used data from 9373 twins $(62.3 \%$ females, mean age $=22.3$ years $)$ in the Twins Early Development Study (TEDS). In each MR-DoC model, the exposure was either a parent- or child-rated mental health measure collected at age 16 years. The instrumental variable was a polygenic score (PS) for the exposure chosen, whereas the outcome was either NSSH or SSH. MR-DoC models were fitted using raw maximum likelihood estimation. We found significant causal effects flowing from child-rated depressive symptoms to both NSSH $(\mathrm{g} 1=0.194$, $95 \%$ CI $0.131,0.257)$ and SSH $(\mathrm{g} 1=0.210,95 \%$ CI $0.125,0.295)$. A similar pattern was observed for causal effects flowing from parentrated depressive symptoms to NSSH $(\mathrm{g} 1=0.092$, 95\% CI 0.004 , $0.181)$ and $\mathrm{SSH}(\mathrm{g} 1=0.165,95 \%$ CI $0.051,0.281)$. All pleiotropic pathways in models with depressive symptoms were significant. There was no significant causal effect flowing from parent-rated ADHD symptoms to NSSH and SSH, but the pleiotropic pathway was significant in the ADHD-SSH model $(\mathrm{b} 2=0.079,95 \% \mathrm{CI}$ $0.027,0.131$ ), and not significant in the ADHD-NSSH model. In terms of causal effects in MR-DoC models, we found no evidence of etiological difference between NSSH and SSH, suggesting they are on the same continuum.

Grant Support: KXL is funded by King's Postgraduate Research International Scholarship.

\section{Genetic and Cultural Transmission of Alcohol Use Disorders in Swedish Twin Pedigrees}

Hermine H. Maes ${ }^{1,2,3,4}$, Henrik Olsson ${ }^{4}$, Paul Lichtenstein ${ }^{5}$, Kristina Sundquist $^{4}$, Jan Sundquist ${ }^{4}$, Kenneth S. Kendler ${ }^{1,2}$

${ }^{1}$ Department of Human and Molecular Genetics, Virginia Institute for Psychiatric and Behavioral Genetics, Virginia Commonwealth University, Richmond VA

${ }^{2}$ Department of Psychiatry, Virginia Institute for Psychiatric and Behavioral Genetics, Virginia Commonwealth University, Richmond VA

${ }^{3}$ Massey Cancer Center, Virginia Commonwealth University, Richmond VA

${ }^{4}$ Center for Primary Health Care Research, Lund University, Malmö, Sweden

${ }^{5}$ Karolinska Institutet, Stockholm, Sweden

Keywords Alcohol use disorders, Twin pedigrees, Genetic transmission, Cultural transmission, Assortment

Using Swedish nationwide registry data, we investigated the contribution of genetic and environmental risk factors to the etiology of alcohol use disorders by extended twin pedigree modeling. Alcohol use disorder (AUD) was defined using public inpatient, outpatient, prescription and criminal records. Three-generational pedigrees were selected for index individuals born between 1980-1990, obtained from the national twin and genealogical registers, whose parents were twins. Relatives of the twins included in the pedigrees were their parents, siblings, spouses and children. Genetic structural equation modeling was applied to the population-based data on AUD, using OpenMx, accounting for the effects of age. Analyses including up to 162,469 individuals in 18,971 pedigrees suggested that prevalence for AUD ranged from 5 to $12 \%$ in males and 2 to $5 \%$ in females. Results predicted substantial heritability ( $\sim 50$ to $60 \%$ ) of which a portion upwards of $5 \%$ is due to the consequences of assortative mating. Contributions of shared environmental factors, which represent a mix of within and cross-generational effects, for AUD appeared to be moderate $(\sim 10$ to $20 \%$ ). Unique environment accounted for the 
remaining variance ( $\sim 20$ to $30 \%$ ). Sex differences in the magnitude of the variance components suggested higher heritability in males and correspondingly higher shared environmental contributions in females. Using objective registry data, we found that AUD is highly heritable. Furthermore, shared environmental factors contributed significantly to the liability of AUD in both males and females.

Grant Support: R01 AA023534.

\section{Triangulating Genetic Methods to Uncover the Contribution of Cognitive and Noncognitive Skills to Academic Achievement Throughout the School Years}

\author{
Margherita Malanchini ${ }^{1,2, *}$, Andrea G. Allegrini ${ }^{3, *}$, Kaili Rimfeld ${ }^{2}$, \\ Michel G. Nivard ${ }^{4}$, Pietro Biroli ${ }^{5}$, Rosa Cheesman ${ }^{6}$, \\ Sophie von Stumm ${ }^{7}$, Perline A. D. Demange ${ }^{4}$, Elsje van Bergen ${ }^{4}$, \\ Andrew D. Grotzinger ${ }^{8}$, Laurel A. Raffington ${ }^{8}$, Javier De la Fuente ${ }^{8}$, \\ K. Paige Harden ${ }^{8}$, Elliot M. Tucker-Drob ${ }^{8}$ \& Robert Plomin ${ }^{2}$ \\ ${ }^{1}$ School of Biological and Chemical Sciences, Queen Mary Univer- \\ sity of London, United Kingdom \\ ${ }^{2}$ Social, Genetic and Developmental Psychiatry Centre, King's Col- \\ lege London, United Kingdom \\ ${ }^{3}$ Department of Psychology, University College London, United \\ Kingdom \\ ${ }^{4}$ Department of Biological Psychology, Vrije Universiteit Amster- \\ dam, Amsterdam, The Netherlands \\ ${ }^{5}$ Department of Economics, University of Zurich, Switzerland \\ ${ }^{6}$ PROMENTA Research Center, Department of Psychology, Univer- \\ sity of Oslo, Oslo, Norway \\ ${ }^{7}$ Department of Education, University of York, United Kingdom \\ ${ }^{8}$ Department of Psychology, The University of Texas at Austin, \\ United States \\ *Joint first authors
}

Keywords Noncognitive skills, Academic achievement, Development, Genomic SEM, Triangulation

Characteristics such as personality, motivation, and socioemotional competencies have been found to account for academic achievement, and its heritability, beyond cognitive skills. These characteristics have been broadly described as noncognitive skills. Several questions regarding the role of noncognitive skills in academic development remain unanswered, including to what extent noncognitive characteristics are associated with academic achievement throughout compulsory education, and the extent to which genetic and environmental factors are implicated in the development of these associations. By triangulating multiple methods, the current study provides a detailed account of how cognitive and noncognitive characteristics are linked to academic performance from age 7 to 16 in the Twins Development Study. Phenotypic and twin analyses showed that noncognitive skills are a fundamental driver of academic achievement, as they predicted variation in achievement at every developmental stage beyond cognitive abilities. Genetic factors accounted for a substantial portion of this prediction. A new polygenic score (PGS) of noncognitive skills, created extending existing approaches to quantify the genetics of noncognitive skills from DNA, significantly predicted variation in noncognitive traits and academic achievement at every developmental stage. While the contribution of cognitive genetics to academic achievement remained relatively stable, the contribution of noncognitive genetics increased substantially over development, providing a glimpse into the mechanisms underlying active gene-environment correlation processes. By placing recent advances in the genetic investigations of noncognitive skills into an educationally relevant, developmental context, the current study provides new crucial insights into the role of cognitive and noncognitive characteristics in academic achievement throughout the school years.

Grant Support: We gratefully acknowledge the ongoing contribution of the participants in the Twins Early Development Study (TEDS) and their families. TEDS is supported by a program grant to RP from the UK Medical Research Council (MR/M021475/1 and previously G0901245), with additional support from the US National Institutes of Health (AG046938).

\section{Enriched X-Chromosome Influences on Neuroanatomical Variation in UK Biobank}

Travis T. Mallard ${ }^{1,2}$, Siyuan $\mathrm{Liu}^{2}$, Jakob Seidlitz ${ }^{2}$, Zhiwei $\mathrm{Ma}^{3}$, Dustin Moraczewski ${ }^{4}$, Adam Thomas ${ }^{4}$, Armin Raznahan ${ }^{2}$

${ }^{1}$ Department of Psychology, University of Texas at Austin, Austin, TX, 78712

${ }^{2}$ Section on Developmental Neurogenomics, Human Genetics Branch, National Institute of Mental Health Intramural Research Program, Bethesda, MD, 20892

${ }^{3}$ Laboratory of Functional and Molecular Imaging, National Institute of Neurological Disorders and Stroke, National Institutes of Health, Bethesda, MD, 20892

${ }^{4}$ Data Science and Sharing Team, National Institute of Mental Health, Bethesda, MD, 20892

Keywords Imaging genetics, Sex differences, Neurodevelopment, Cognition, Intellectual disability

The X-chromosome has long been hypothesized to have a disproportionate influence on the brain, as genes located on chromosome often show enriched expression in brain tissue relative to other chromosomes (Nguyen \& Disteche, 2006). Moreover, it has been demonstrated that the $\mathrm{X}$-chromosome is enriched for genes that cause intellectual disability when mutated (Zechner et al., 2001). Here, we verify this hypothesis through partitioned heritability analysis of $\mathrm{X}$-chromosome influences (XIs) on human brain anatomy in 32,256 individuals from the UK Biobank. We first establish evidence for dosage compensation in XIs on brain anatomy, reflecting larger XIs in males as compared to females. Moreover, we find that the spatial pattern of dosage compensation across the cortex is correlated with that of regional sex-differences in neuroanatomical variance. We then demonstrate that XIs are significantly larger than would be predicted from X-chromosome size for the relative surface area of cortical systems supporting attention, decision making, and motor control. Finally, we report several follow-up analyses that implicate X-linked genes with pleiotropic effects on neuroanatomy and cognition. Taken together, our findings reveal a privileged role for the X-chromosome in human neurodevelopment, and urge greater inclusion of this chromosome in future genetically informative research.

\section{References}

Nguyen, D. K. \& Disteche, C. M. Dosage compensation of the active $\mathrm{X}$ chromosome in mammals. Nat. Genet. 38, 47-53 (2006).

Zechner, U. et al. A high density of X-linked genes for general cognitive ability: a run-away process shaping human evolution? Trends Genet. 17, 697-701 (2001).

Grant Support: This research was supported by the Intramural Research Program of the National Institute of Mental Health (NIH Annual Report Number, ZIA MH002949-04). 
Perspectives of the Gender Roles in the Family Versus Personality Traits

Sherry Mao ${ }^{1}$, Yoo Ri Hwang ${ }^{1}$, Jonathan Trattner $^{1}$, S. Mason Garrison $^{1}$

${ }^{1}$ Department of Psychology, Wake Forest University, Winston Salem, NC, USA

Keywords Big Fiver Personality, Gender

Attitudes toward gender roles have shifted in recent decades (Fortin, 2005), but they still vary considerably (Sani and Quaranta, 2017). These attitudes affect how people behave as well as how they perceive others' behavior in practically every social situation (e.g., Eccles, 1987). Given that these attitudes develop at a young age (Beal 1994) and are transmitted across generations (Farré \& Vella, 2012), it is important to understand the stable factors that influence them, such as personality. Using the 1979 National Longitudinal Survey of Youth, we examined the relationship between attitudes about gender roles and personality traits. To do so, we used the discordant-kinship model (Garrison \& Rogers, 2016) which enabled us to control for gene-and-environmental variance among 1,278 adult sibling pairs and allowed us to better understand the personality-attitude relationship. Preliminary results suggest that sibling differences in big five traits are significantly associated with differences in gender role attitudes.

References

Beal, C. R. (1994). Boys and girls: the development of gender roles. McGraw-Hill.

Dotti Sani, G. M., \& Quaranta, M. (2016). The Best Is Yet to Come? Attitudes Toward Gender Roles Among Adolescents in 36 Countries. Sex Roles, 77(1-2), 30-45. https://doi.org/10.1007/s11199-016-0698-7

Eccles, J. S. (2006, July 28). Gender roles and women's achievementrelated decisions. https://doi.org/10.1111/j.1471-6402.1987.tb00781.x. Farré, L., Klein, R., \& Vella, F. (2011, September 6). Does Increasing Parents' Schooling Raise the Schooling of the Next Generation? Evidence Based on Conditional Second Moments*. Wiley Online Library. https://doi.org/10.1111/j.1468-0084.2011.00667.x.

Fortin, J. (2005). Première ligne : https://doi.org/10.1522/24115069

Garrison, S. M., \& Rodgers, J. L. (2017). Decomposing the causes of the Socioeconomic Status-Health gradient WITH Biometrical Modeling. https://doi.org/10.31219/osf.io/xbkja

\section{Developmental cascades from polygenic and prenatal substance use to adolescent substance use: Leveraging severity and directionality of externalizing and internalizing problems to understand pubertal and harsh discipline-related risk}

\author{
Kristine Marceau ${ }^{1}$, Gregor Horvath ${ }^{2}$, Amy Loviska ${ }^{1}$, \\ Valerie S. Knopik ${ }^{1}$ \\ ${ }^{1}$ Department of Human Development and Family Studies, \\ Purdue University, West Lafayette, Indiana, USA \\ ${ }^{2}$ Rogers Behavioral Health, Oconomowoc, WI \\ Keywords ALSPAC, Adolescent substance use, Externalizing, \\ Polygenic, Prenatal substance use, Pubertal timing
}

We tested a developmental cascade from puberty-related polygenic risk and prenatal substance use through pubertal timing and parenting to the severity and directionality (i.e., differentiation) of externalizing and internalizing problems to adolescent substance use in $n=4504$ White boys, $n=4287$ White girls from the Avon Longitudinal Study of Parents and Children (ALSPAC) cohort. Puberty-related polygenic risk was calculated using summary statistics from the largest available GWAS on age at menarche. Prenatal substance use was a weighted severity score of smoking, alcohol, and cannabis use frequency maternallyreported prior to 18 week and at 32 week gestation. Parental harsh discipline was parent-reported at 9.5-11.5 years. Pubertal timing was the age of reaching Tanner Stage 2 from repeated measures of parentand youth-reports. Severity and directionality of internalizing and externalizing problems was parent-reported at 13 years. Alcohol, smoking, and cannabis use were measured on continuums of substancespecific stages increasing in quantity and risk for dependence/abuse youth-reported at 18.5 years. Results from path models found that girls' earlier pubic hair development timing mediated the PRS for puberty and differentiation towards internalizing symptoms. Limited associations of early pubertal timing with substance use outcomes were only observable via symptom directionality, differently for girls and boys. For boys, exposure to prenatal substance use predicted substance use via differentiation towards relatively more pure externalizing problems, but in girls associations were largely direct. Severity and especially differentiation towards externalizing problems were key intermediaries in developmental cascades from parental harsh discipline with substance use progressions for girls and boys.

Grant Support: K01 DA039288, NIDA.

\section{Autism Spectrum Disorders and Increased Brain Volume Link Through a Set of mTOR-Related Genes}

Arenella Martina ${ }^{1,2,3}$, Mota, Nina Roth ${ }^{1,3}$, Teunissen, Mariel W.A. ${ }^{1,4}$, PhD, Brunner, Han G. ${ }^{1,5,6}$, Bralten, Janita ${ }^{1,3}$.

${ }^{1}$ Department of Human Genetics, Radboud university medical center, Nijmegen, The Netherlands

${ }^{2}$ Department of Forensic and Neurodevelopmental Science, Institute of Psychiatry, Psychology and Neuroscience, King's College London, London, The United Kingdom

${ }^{3}$ Donders Institute for Brain, Cognition and Behaviour, Radboud University, Nijmegen, The Netherlands

${ }^{4}$ Department of Neurology, Maastricht university medical centre, Maastricht, The Netherlands

${ }^{5}$ Department of Clinical Genetics, Maastricht University Medical centre, Maastricht, The Netherlands

${ }^{6}$ GROW School of development and Oncology, and MHENS school of Neuroscience, Maastricht University, The Netherlands

Keywords Autism spectrum disorders, Brain volume, Genetic correlation, mTOR gene-set

Large brains are often observed in individuals with autism spectrum disorders (ASDs). Both ASDs and brain volume are highly heritable, and it is unclear if these traits share genetic mechanisms. Genes from the mammalian target of rapamycin (mTOR) pathway influence variability in brain volume, and mutations are found in rare genetic syndromes that include ASD features. We investigated whether variations in mTORrelated genes constitute a genetic link between large brains and ASDs. To test this hypothesis, we first investigated whether macrocephaly and ASDs were enriched in individuals with rare de-novo mTOR-related variants in a local cohort $(\mathrm{N}=2257)$ and using publicly available data $(\mathrm{N}$ $=32,991)$. We next explored common variant datasets and estimated the genetic correlation between ASDs $(\mathrm{N}=46,350)$ and ICV $(\mathrm{N}=25,974)$ by LD-score regression. Lastly, we performed gene-set analyses in MAGMA and restricted the ASD-ICV genetic correlation analysis to variants in mTOR-related genes using GNOVA. Both macrocephaly and ASDs are more frequent in individuals carrying rare de-novo mutations in mTOR-related genes compared to carriers of de-novo non -mTOR-related mutations. Although no significant genome-wide correlation 
between ASD and ICV was present $(p=0.81)$, we show a significant mTOR gene-set association with ASDs $(p=0.0029)$, and a mTORstratified positive genetic correlation between ASD and ICV $(p=0.027)$.

\section{Genome-Wide Association Study of Food Liking in 162,000 People Uncovers the Genetic Bases of Food Liking}

Sebastian May-Wilson ${ }^{1}$, Nana Matoba ${ }^{2,3}$, Kaitlin Wade $^{4}$, Maria Pina Concas ${ }^{5}$, Jouke-Jan Hottenga ${ }^{6}$, Massimo Mangino ${ }^{7,8}$, Maria G. Veldhuizen ${ }^{9}$,Cristina Menni ${ }^{8}$, Eco de Geus ${ }^{6,10}$, Paolo Gasparini ${ }^{5,9}$, Nicholas Timpson ${ }^{4}$, Jim Wilson ${ }^{1,11}$, Nicola Pirastu ${ }^{1}$

${ }^{1}$ Centre for Global Health Research, Usher Institute, University of Edinburgh, Scotland UK

${ }^{2}$ Department of Genetics, University of North Carolina at Chapel Hill, Chapel Hill, NC, USA

${ }^{3}$ UNC Neuroscience Center, University of North Carolina at Chapel Hill, Chapel Hill, NC, USA

${ }^{4}$ MRC Integrative Epidemiology Unit (MRC-IEU), University of Bristol, England UK

${ }^{5}$ Institute for Maternal and Child Health - IRCCS "Burlo Garofolo",Trieste, Italy

${ }^{6}$ Department of Biological Psychology, Faculty of Behavioral \& Movement Sciences, Vrije Universiteit, Amsterdam

${ }^{7}$ Department of Twin Research and Genetic Epidemiology, King's College London, London, UK

${ }^{8}$ NIHR Biomedical Research Centre at Guy's and St Thomas' Foundation Trust, London UK

${ }^{9}$ Department of Medical Sciences, University of Trieste, Trieste, Italy

${ }^{10}$ Amsterdam Public Health research institute, Amsterdam University Medical Centers, The Netherlands

${ }^{11}$ MRC Human Genetics Unit, Institute of Genetics and Cancer, University of Edinburgh, Scotland UK

\section{Keywords Food liking, GWAS, GenomicSEM}

Variable preferences for different foods are among the main determinants of their intake and are influenced by many factors including genetics. Despite $\sim 50 \%$ heritability, studies aimed at uncovering food liking genetics have focused mostly on taste receptors. Here we present the first results of a large-scale genome-wide association study of food liking conducted on 161,625 participants from UK-biobank. Liking was assessed over 142 specific foods using a 9-point Likert scale. After performing GWAS we used genetic correlation coupled with structural equation modelling to create a multi-level hierarchical map of food liking which identified 3 main dimensions: high caloric foods defined as "innate", strong tasting foods ranging from alcohol to strong tasting vegetables defined as "learned" and finally "low caloric" foods such as fruit and vegetables. The "innate" dimension was genetically completely independent from the other two suggesting that two independent processes underlie to liking high reward foods from the learned/low caloric ones. Genetic correlation analysis with corresponding food consumption traits evidenced a high correlation $(\sim 0.75)$ while showing twice the heritability. GWAS analysis identified 1401 food liking-SNP associations located in 173 genomic loci several of which close to taste or olfactory receptors. Genetic correlation with morphological and functional brain data $(33,224$ participants) results in associations of the three liking dimensions with non-overlapping, distinct brain areas and networks, suggestive of separate neural mechanisms underlying the liking determinants. In conclusion, we created a comprehensive and data-driven map of genetic determinants and associated neurophysiological factors of food liking beyond taste receptor genes.

\section{Using Polygenic Risk Scores to Dissect Heterogeneity Between Major Depressive Disorder Subtypes}

Brittany L. Mitchell ${ }^{1,2}$, Adrian I Campos ${ }^{1,3}$, David C Whiteman ${ }^{1}$, Catherine M Olsen ${ }^{1}$, Scott D. Gordon ${ }^{1}$, Adam Walker ${ }^{4}$, Olivia M Dean ${ }^{4,5}$, Michael Berk ${ }^{4,5,6}$, Ian B Hickie ${ }^{7}$,

Naomi R Wray ${ }^{8,9}$, Sarah E Medland ${ }^{1}$, Nicholas G Martin ${ }^{1}$, Enda M Byrne ${ }^{8}$

${ }^{1}$ QIMR Berghofer Medical Research Institute, Brisbane, Australia

${ }^{2}$ School of Biomedical Sciences, Faculty of Health, Queensland University of Technology (QUT) Brisbane, Australia

${ }^{3}$ School of Biomedical Sciences, Faculty of Medicine, The University of Queensland, Brisbane, Australia.

${ }^{4}$ Deakin University, Institute for Mental and Physical Health and Clinical Translation, School of Medicine, Barwon Health, Geelong, Australia

${ }^{5}$ Florey Institute for Neuroscience and Mental Health and the Department of Psychiatry, The University of Melbourne, Melbourne, Australia

${ }^{6}$ Orygen, The National Centre of Excellence in Youth Mental Health, Centre for Youth Mental Health

${ }^{7}$ Brain and Mind Centre, The University of Sydney, Sydney, New South Wales, Australia

${ }^{8}$ Institute for Molecular Bioscience, The University of Queensland, Brisbane, Australia

${ }^{9}$ Queensland Brain Institute, The University of Queensland, Brisbane, Australia

Keywords Major depressive disorder, Genetics, Heterogeneity, Psychiatry, Polygenic risk scores

Major depressive disorder (MDD) is a common and highly heterogeneous psychiatric disorder but little is known about the genetic characterization of this heterogeneity. Understanding the genetic etiology of MDD can be challenging as large sample sizes are needed for gene discovery but this is often achieved with a trade-off in the depth phenotyping. The Australian Genetics of Depression Study is the largest stand-alone depression cohort with both genetic data and in-depth phenotyping and comprises a total of 15,792 participants of European ancestry, $92 \%$ of which, met diagnostic criteria for MDD. We leveraged the unique nature of this cohort to investigate genetic heterogeneity across various clinical subtypes of MDD using polygenic risk scores (PRS). We show that a PRS for depression explains $5.7 \%$ of variance in MDD liability in our sample and find strong support for genetic heterogeneity in depression with differential associations of multiple psychiatric and comorbid traits with age of onset, longitudinal course and various clinical subtypes of MDD. Until now, this degree of detailed phenotyping in such a large sample of MDD cases has not been possible. In this study, we provide support for differential pathways to illness models that recognize both the overlap with other common psychiatric disorders, as well as pathophysiological differences.

\section{Polygenic Liability for ADHD but not ASD Relates to Infant Temperament}

Emma L. Meaburn ${ }^{1,2}$, Anna Gui ${ }^{1,2}$, Rebecca Harrison ${ }^{2}$, Greg Pasco ${ }^{3}$, Jannath Begum Ali ${ }^{1}$, Hamel Patel ${ }^{4}$, Charles Curtis ${ }^{4}$, Tony Charman ${ }^{3}$, Mark H. Johnson ${ }^{5}$, Emily J. H. Jones ${ }^{1,2}$ and the BASIS Team ${ }^{1}$

${ }^{1}$ Centre for Brain and Cognitive Development, Birkbeck College, University of London, Malet Street, WC1E 7HX, United Kingdom

${ }^{2}$ Department of Psychological Sciences, Birkbeck College, University of London, Malet Street, WC1E 7HX, United Kingdom 
${ }^{3}$ Department of Psychology, Institute of Psychiatry, Psychology \& Neuroscience, King's College London, De Crespigny Park, London SE5 8AF, United Kingdom

${ }^{4}$ NIHR BioResource Centre Maudsley, King's College London, London SE5 8AF, United Kingdom

${ }^{5}$ Department of Psychology, Cambridge University, Downing Street, Cambridge CB2 3EB, United Kingdom

Keywords Autism, Polygenic scores, Infancy, Development, Temperament

Temperament in early life can be conceptualised as variability in activity, affectivity, attention, and self-regulation, and is linked to neurobiological systems and later psychopathology. Here, we investigate whether individual differences and developmental trajectories of temperament are an early behavioral manifestation of polygenic liability for autism spectrum disorder (ASD) and ADHD in infants with and without a family history of ASD. Polygenic scores for ASD and ADHD were calculated using PRSice- 2 for 236 infants of European ancestry with $(\mathrm{FH}, \mathrm{n}=172)$ and without $(\mathrm{noFH}, \mathrm{n}=64)$ a family history of ASD enrolled in a prospective longitudinal study (BASIS). Parent-reported temperament was obtained longitudinally from 10 to 36 months using age-appropriate questionnaires (Infant Behavior Questionnaire-Revised; Early Childhood Behavior Questionnaire; Children's Behavior Questionnaire). Diagnostic assessment for ASD was performed at 36 months. We examined the association between (1) temperament and ASD (2) PGS $_{\mathrm{ASD}}$ and PGS $\mathrm{ADHD}_{\mathrm{A}}$ and the domains of surgency, negative effect and effortful control at 36 months, and (3) longitudinal trajectories of temperament domains. The examined temperament domains differed between infants with and without ASD (all $\mathrm{p}<0.05)$. Higher $\mathrm{PGS}_{\mathrm{ADHD}}\left(\mathrm{P}_{\mathrm{T}}=0.01\right)$, but not $\mathrm{PGS}_{\mathrm{ASD}}$, was associated with higher negative affect $(\beta=0.17, \mathrm{SE}=$ $0.06, p=0.004)$, and lower effortful control $(\beta=-0.13, \mathrm{SE}=0.05, \mathrm{p}$ $=0.014)$ at 36 months. Trajectory analyses show distinct profiles of temperament that will be linked to PGS $_{\mathrm{ADHD}}$ in further analysis. Our emerging findings support research linking early temperament with later ASD and indicates negative emotionality and effortful control in infants with a family history of neurodevelopmental disorders relates to ADHD (but not ASD) genetic liability.

Grant Support: The genetic data generation was funded by the Simons Foundation Autism Research Initiative (SFARI) Pilot Award [grant No 511504] to E.J.H.J and E.L.M. The experimental and behavioral data collection for all Phases of BASIS was funded by MRC Programme [grant No G0701484 and MR/K021389/1], the BASIS funding consortium led by Autistica (www.basisnetwork.org).

\section{Exploring the Archives: A review of Pre-Existing Longitudinal Family Studies with Life Outcomes}

Morgan Milhollen $^{1,2}$, S. Mason Garrison ${ }^{2}$

${ }^{1}$ Department of History, Wake Forest University Winston-Salem, North Carolina, USA

${ }^{2}$ Department of Psychology, Wake Forest University Winston-Salem, North Carolina, USA

\section{Keywords Longitudinal Studies, Pre-Existing Data, Pedigrees}

Although worthwhile, longitudinal studies are time-consuming and expensive. Furthermore, they are not always economically feasible or global events. Thus, repurposing pre-existing data is invaluable. However, many scholars are unaware that genetically informed data exists in public databases. Therefore, we aimed to understand better what family and pedigree data exist. To explore this data, we searched ICPSR and Harvard-Dataverse using terms including "family-trees," "pedigree," and "life outcomes." We limited our search to studies with at least three generations with life outcomes. Although the systematic review is ongoing, we have identified studies from many countries (e.g., USA, China, Netherlands), with many outcomes (e.g., marriage, fertility, mortality, health) and contexts (e.g., geography, weather, culture, SES, century). Although pre-existing data have some notable disadvantages (including missing data), these preexisting datasets can include information on hundreds of thousands of individuals, spanning over 100 years, and thus contain more entries than any one researcher can collect in their lifetime. Indeed, they allow psychologists to study populations beyond their lifetime. In addition to these general observations, we will highlight the China Multi-Generational Panel Datasets (Campbell \& Lee, 2017; Lee \& Campbell, 2016), which provide data on five generations of individuals.

\section{References}

Campbell, Cameron D., and Lee, James Z. China Multi-Generational Panel Dataset, Shuangcheng (CMGPD-SC), 1866-1913. ICPSR, 2017-11-03. https://doi.org/10.3886/ICPSR35292.v8

Lee, James Z., and Campbell, Cameron D. China Multi-Generational Panel Dataset, Liaoning (CMGPD-LN), 1749-1909. ICPSR, 2016-0906. https://doi.org/10.3886/ICPSR27063.v10

Grant Support: Not Applicable

\section{Testing Association of Previously Implicated Gene-Sets in Nicotine Exposed Mouse Models with Human Smoking Phenotypes}

Travis J. Mize ${ }^{1,2}$, Scott A. Funkhouser ${ }^{1}$, Jordan M. Buck ${ }^{1,3}$, Jerry A. Stitzel ${ }^{1,3}$, Marissa A. Ehringer ${ }^{1,3}$, \& Luke M. Evans ${ }^{1,2}$ ${ }^{1}$ Institute for Behavioral Genetics, University of Colorado, Boulder, CO, USA

${ }^{2}$ Department of Ecology and Evolutionary Biology, University of Colorado, Boulder, CO, USA

${ }^{3}$ Department of Integrative Physiology, University of Colorado, Boulder, CO, USA

\section{Keywords Genetics, Smoking, GWAS, MAGMA, GSCAN}

Tobacco use leads to more than eight million deaths per year worldwide, with cigarette smoking being the single largest contributor. Smokingrelated behaviors are partly heritable, yet the genetic contribution to human smoking phenotypes is still not fully understood; however, nicotine exposure mouse models have implicated numerous genes that may be involved in risk for certain behaviors among offspring. Here, we sought to examine whether previously identified genes that are differentially expressed in D1-type striatal medium spiny neurons in developmental nicotine exposure (DNE) mice are associated with human smoking behaviors. Summary statistics from a large GWAS (GSCAN) of four phenotypes (age of smoking initiation, cigarettes per day, smoking cessation, and smoking initiation) were analyzed in conjunction with multi-marker analysis of genomic annotation, or MAGMA. Of the genes contained within each gene-set, TMEM18 and TOX were found to be statistically significant $(\mathrm{p}<5 \mathrm{e}-8)$ at the genelevel in the smoking initiation GWAS. However, this set of differentially expressed genes in DNE mice were no more strongly associated with human smoking behaviors than the rest of the genome. This might not be surprising, given that these were identified as differentially expressed genes in a single cell type due to an environmental exposure 
and therefore may not represent innate genetic differences. Ongoing analyses include applying stratified LD Score Regression to estimate the heritable contribution of this set of genes and assessing whether the association signal of SNPs within these genes is stronger when only examining those variants that also contribute to expression variation in humans.

\section{Methods for Enabling and Improving the Power of Mendelian Randomization Studies of Parental Environmental Exposures and Offspring Outcomes}

Gunn-Helen Moen 1,2,3,4, Laxmi Bhatta ${ }^{3}$, Bjørn Olav Åsvold ${ }^{3,6}$, Ben Brumpton ${ }^{3,7,8, *}$, Nicole M Warrington ${ }^{2,3, *}$, David M. Evans ${ }^{1,5, *}$ ${ }^{1}$ Institute of Clinical Medicine, Faculty of Medicine, University of Oslo, Oslo, Norway

${ }^{2}$ The University of Queensland Diamantina Institute, The University of Queensland, Brisbane, Australia

${ }^{3}$ K.G. Jebsen Center for Genetic Epidemiology, Department of Public Health and Nursing, NTNU, Norwegian University of Science and Technology, Trondheim, Norway

${ }^{4}$ Population Health Sciences, Bristol Medical School, University of Bristol, Bristol, UK

${ }^{5}$ Medical Research Council Integrative Epidemiology Unit, University of Bristol, Bristol, UK

${ }^{6}$ Department of Endocrinology, Clinic of Medicine, St. Olavs Hospital, Trondheim University Hospital, Trondheim, Norway

${ }^{7}$ Clinic of Medicine, St. Olavs Hospital, Trondheim University Hospital, Trondheim, Norway

${ }^{8}$ HUNT Research Center, Department of Public Health and Nursing, NTNU, Norwegian University of Science and Technology, Levanger, Norway

*These authors jointly supervised this work.

Keywords Mendelian randomization, Parent-offspring pairs, Birthweight, Norwegian HUNT cohort

There is growing interest in using Mendelian randomization (MR) to investigate the causal effect of parental influences on their offspring. However, a major obstacle is the paucity of large-scale cohorts around the world that have genotype information on mother-offspring pairs or parent-offspring trios. Another difficulty is that MR studies in this context often rely on simple regression models that may not be optimal in terms of statistical power. In this talk we summarize our efforts to devise techniques to facilitate MR analyses of summary results data in this context, and methods to improve the power of MR analyses when complete data on mother-offspring pairs/parent-offspring trios is available. We illustrate how genomic SEM can be used to combine estimates of maternal, paternal and offspring genetic effects that are derived from different data sources where the extent of relatedness and sample overlap is unknown, and subsequently use these in MR analyses. We also show how the genetic linear mixed model can be extended to include genetic relationship matrices indexing offspring, maternal, paternal similarity as well as the covariance between them in order to model error variance and provide more precise estimates of genetic effects for subsequent MR analyses. Finally, we illustrate these methods by using them to estimate the causal effect of parental phenotypes on first and second born offspring birthweight using summary results data from 39,432 mothers, 34,095 fathers and 10,066 offspring from the Norwegian HUNT cohort as well as 5725 complete parent-offspring trios.

Grant Support: G.H.M. is supported by the Norwegian Research Council (Post doctorial mobility research grant 287198). N.M.W. is supported by an Australian National Health and Medical Research Council Early Career Fellowship (APP1104818). D.M.E. is funded by an Australian National Health and Medical Research Council Senior Research Fellowship (APP1137714) and NHMRC project grants (GNT1125200, GNT1157714, GNT1183074).

\section{Do Parenting Styles and Parental Involvement in School Actually Influence School Performance? The Role and Importance of Common Genetic and Common Environmental Influences}

Bastian Mönkediek ${ }^{1}$, Martin Diewald ${ }^{1}$

${ }^{1}$ Faculty of Sociology, Bielefeld University, Universitätsstraße 25, 33615 Bielefeld, Germany

Keywords Math grades, Parenting styles, Parenting practices, Twins, Germany

Differences in children's school performance are often associated with differences in parents' parenting styles and school involvement practices, which are often seen as part of the mechanisms by which parents can pass on social disadvantages to children. However, there are doubts about the extent to which the observable relationships are causal or reflect genetic influences. To trace the effective influence of parenting styles and involvement practices on school performance, we examine what underlies the existing associations and to what extent they are driven by common genetic and common environmental influences, while additionally including the possibility of moderation effects. The analysis is based on the first two waves of the German Twin Family Panel (TwinLife), a random sample of twin families from all parts of Germany. We focus on twins $(\mathrm{N}=2,056)$ aged 10 to 12 years old at the time point of the first interview and relate the parenting they received to their math grades when they were 10 to 12 and 12 to 14 years old. Our analyses here are based on the full bivariate moderation model proposed by Purcell. Our results demonstrate that the covariance between parental involvement practices and math grades largely relates to common genetic influences, hinting for processes related to gene-environment correlation (rGE). For parenting styles, however, the covariance is mainly due to common, non-shared environmental influences. Our results thus hint for differences in the underlying processes for parenting styles and involvement practices and for possible differences in the causality of relationships.

Grant Support: This work is supported by funding from the German Research Foundation (DFG) as long-term project TwinLife (DI 759/11-3).

\section{Polygenic Prediction of Cognitive Abilities Between and Within Family Members}

Claire L. Morrison ${ }^{1}$, Matthew C. Keller ${ }^{1}$, Chandra A. Reynolds ${ }^{2}$, Sally J. Wadsworth ${ }^{1}$, Robin P. Corley ${ }^{1}$, Naomi P. Friedman ${ }^{1}$

${ }^{1}$ Institute for Behavioral Genetics, University of Colorado Boulder, Boulder, CO

${ }^{2}$ Department of Psychology, University of California, Riverside

Keywords Executive function, IQ, Reaction time, PRS, EF

Disentangling how genes and environments combine to influence outcomes is fundamental to the field of behavioral genetics. Genome- 
wide association studies (GWAS) of unrelated individuals quantify allelic associations that may reflect both direct genetic effects as well as indirect genetic effects ("genetic nurture" from rearing environments). Recent work separating effects of polygenic scores into within- and between-family effects has shown that cognitive traits may be especially likely to be impacted by indirect genetic effects in addition to direct effects. Family data are useful for studying these effects because within-family effects control for shared environmental influences, whereas between-family genetic effects may be inflated by assortative mating or gene-environment correlations. Indeed, comparisons of within- vs. between-family effects for polygenic scores suggest indirect genetic effects for cognition-related traits such as educational attainment and intelligence, in contrast to more biological traits like height that show predominately direct genetic effects. However, the range of cognitive phenotypes examined so far has been limited, focusing primarily on intelligence and educational attainment. Here we investigate whether different cognitive constructs, particularly executive functioning and speed, show patterns consistent with indirect genetic effects that are similar to the patterns obtained with intelligence and educational attainment. Using GWAS summary statistics for the UK Biobank sample, we calculated polygenic scores for common executive functioning, intelligence, speed, and educational attainment in a sample of $\sim 300$ dizygotic twin pairs from two Colorado twin studies. Then we used mixed models to parse their effects on cognitive outcomes into between and within-family effects.

Grant Support: National Institutes of Health Grants AG046938, MH016880, MH063207, and MH001865.

\section{Genetic Prejudice as a Cognitive Bias: Evidence from a Multivariate Twin Study}

\author{
José J. Morosoli ${ }^{1,2}$, Lucía Colodro-Conde ${ }^{1}$, Fiona K. Barlow ${ }^{2}$, \\ Sarah E. Medland ${ }^{1}$ \\ ${ }^{1}$ Psychiatric Genetics Group, QIMR Berghofer Medical Research \\ Institute, Brisbane, Australia \\ ${ }^{2}$ School of Psychology, University of Queensland, Brisbane, Australia
}

Keywords Prejudice, Need for closure, Genetic determinism, Moral values, Twin study

Learning about someone's genetic predisposition could prompt prejudiced behaviors in others. For example, 20-25\% of our survey participants declare that they would not choose a partner who has a strong genetic predisposition for depression. Previous research in social psychology suggests that prejudiced behavior could stem from an aversion towards ambiguity in social contexts. Moreover, genetic deterministic thinking has also been associated with both need for firm answers and prejudice, as well as with conservative ideologies. Need for certainty has been proposed as the general cognitive disposition underlying this type of categorical thinking. It has also been hypothesized that need for certainty, prejudiced thinking, and conservatism may be the product of overreliance on certain "innate" heuristics (i.e., mental shortcuts that allows make judgments quickly). Arguably, this could also be the case for genetic deterministic thinking. In the present study, we surveyed 3974 Australian twins on genetic deterministic thinking, reliance on heuristics, need for certainty, conservative values, and attitudes towards partners with strong genetic predisposition for depression. Using a multivariate twin design, we tested the hypothesis of an innate cognitive mechanism underlying all five measures. Heritability was low to moderate $\left(\mathrm{H}^{2}=\right.$ $16-54 \%)$. Phenotypic correlations between measures were low to moderate $(r=0.01-0.20)$. Genetic determinism showed little genetic influence and its genetic correlation with cognitive measures was nonsignificant. Genetic correlations were significant between need for certainty, conservatism, and heuristic thinking, supporting the hypothesis of shared innate mechanisms. Implications for research in prejudice and public understanding of genetics will be discussed.

Grant Support: This study was funded by the John Templeton Foundation (Genetics and Human Agency Project).

\section{Understanding the Genetics of Manic Symptoms in the Genetic Links to Anxiety and Depression Study}

Jessica Mundy ${ }^{1}$, Christopher Hübel ${ }^{1,2,3}$, Jonathan R I Coleman ${ }^{1,2}$, Evangelos Vassos ${ }^{1,2}$, Robin M Murray ${ }^{2,4}$, and Gerome Breen ${ }^{1,2}$

${ }^{1}$ Social, Genetic and Developmental Psychiatry Centre; Institute of Psychiatry, Psychology \& Neuroscience, King's College London, London, United Kingdom

${ }^{2}$ UK National Institute for Health Research (NIHR) Biomedical Research Centre, South London and Maudsley National Health Service (NHS) Trust, London, United Kingdom

${ }^{3}$ National Centre for Register-based Research, Aarhus Business and Social Sciences, Aarhus University, Aarhus, Denmark

${ }^{4}$ Institute of Psychiatry, Psychology \& Neuroscience, King's College London, London, United Kingdom

Keywords Bipolar disorder, Mania, Factor analysis

Bipolar disorder is a heritable psychiatric disorder. Individuals with bipolar disorder experience swings from depressed states to mania in bipolar type I and hypomania states in bipolar type II. Mania can be measured using the Mood Disorder Questionnaire (MDQ) which asks about the presence or absence of 13 manic symptoms. The aims of this study were first, to perform factor analysis on the items from the MDQ to understand the underlying latent structure of the mania. Second, to perform genome-wide association studies (GWASs) of (a) factors underlying the structure of the MDQ and (b) a continuous mania trait to understand their genetic overlap with other psychiatric traits. This study is based on participants in the Genetic Links to Anxiety and Depression Study which is part of the NIHR Bioresource and collects both phenotypic and genetic data. We conduct exploratory and confirmatory factor analysis on the binary answers to the MDQ and compute factor scores based on the underlying factor structure. Based on the participants' genetic data, we perform GWAS of the factor scores, as well as a continuous mania trait (based on the 13 items), controlling for the first 10 ancestry principal components, genotyping batch, sex and age. Using the summary statistics produced by the GWASs, we compute genetic correlations with other psychiatric traits. A three factor model fit the data best. These factors can be broadly defined as: impulsivity, hyperactivity and sociability. GWAS of the factors, as well as a continuous mania trait, are currently being performed. Heritability and genetic correlation results will be available shortly.

Grant Support: This work was supported by the NIHR Biomedical Research Centre (BRC) and the Lord Leverhulme Charitable Grant.

\section{Scrutinizing the Genetic Overlap Between Disorders Characterized by Disturbed Water and Ion Homeostasis in the Brain}

Gema Muñoz ${ }^{1}$, Rogier Min ${ }^{2,3}$, Mats Nagel ${ }^{1}$, Sophie van der Sluis ${ }^{1,4}$ 
${ }^{1}$ Department of Complex Trait Genetics, Center for Neurogenomics and Cognitive Research, Amsterdam Neuroscience, Vrije Universiteit Amsterdam, Amsterdam, The Netherlands

${ }^{2}$ Department of Child Neurology, Amsterdam Neuroscience, VU University Medical Center, Amsterdam, The Netherlands

${ }^{3}$ Department of Integrative Neurophysiology, Center for Neurogenomics and Cognitive Research, Amsterdam Neuroscience, VU University, Amsterdam, The Netherlands

${ }^{4}$ Department of Child and Adolescence Psychiatry, section Complex Trait Genetics, Amsterdam UMC, Amsterdam, The Netherlands

Keywords Brain ion and water homeostasis, GWAS, Summary statistics, Genetic correlation

Megalencephalic Leukoencephalopathy with Subcortical Cysts (MLC) is a rare infantile-onset leukodystrophy caused by monogenic mutations in $M L C 1$ or GLIALCAM. MLC patients present impaired brain ion and water homeostasis, which can lead to e.g. progressive neurological deterioration. Interestingly, MLC patients with the same mutation can present highly heterogeneous clinical courses, suggesting the existence of additional genetic or environmental factors influencing brain ion and water regulation. To further examine the genetic mechanisms underlying these processes, we analyzed the genetic overlap between the following traits, which are biologically related to ion/water homeostasis: overall brain volume, grey and white matter volume, two white matter microstructure-derived parameters, stroke outcome, and epilepsy risk. SNP-based heritability of these traits ranged from $22.14 \%$ and $40.97 \%$, except for stroke outcome, for which the heritability was approximately zero, probably due to small sample size. Genetic correlations between these traits ranged between 0.003 and 0.884 . Analysis of local genetic correlations yielded further insight into the shared genetic architecture of these traits. Cross-trait meta-analysis yielded 62 significant genes, among which $V C A N$, which encodes a chondroitin sulfate proteoglycan influencing e.g. water content in several tissues. MAGMA gene-set analysis on the cross-trait meta-analytic results showed significant association with two gene-sets: PI3K/Akt signaling in cancer, and insulin receptor substrate binding. These analyses identified genes that are jointly associated to traits characterized by ion/ water imbalance. Whether variation in these genes explains the phenotypic heterogeneity observed in MLC patients, merits further research

Grant Support: This work was partially supported by NWO Gravitation program BRAINSCAPES: A Roadmap from Neurogenetics to Neurobiology (NWO: 024.004.012).

\section{Dynamic Complementarity in Skill Production: Evidence from Genetic Endowments and Birth Order}

Dilnoza Muslimova ${ }^{1,4}$, Hans van Kippersluis ${ }^{1,4}$,

Cornelius A. Rietveld ${ }^{1,2,4}$, Stephanie von Hinke ${ }^{1,3,4}$, S. Fleur W. Meddens ${ }^{1,2}$

${ }^{1}$ Erasmus School of Economics, Erasmus University Rotterdam, Rotterdam, The Netherlands

${ }^{2}$ Erasmus University Rotterdam Institute for Behavior and Biology, Erasmus University Rotterdam, Rotterdam, The Netherlands

${ }^{3}$ School of Economics, University of Bristol, Bristol, United Kingdom; ${ }^{4}$ Tinbergen Institute, Amsterdam, The Netherlands

Keywords Birth order, Dynamic complementarity, Gene-environment interaction, Educational attainment, Polygenic score
On average, firstborns complete more education than their laterborn siblings. We study whether this effect is amplified by genetic endowments. Our family-fixed effects approach allows us to exploit exogenous variation in birth order and genetic endowments among 14,850 siblings in the UK Biobank. We find that those with higher genetic endowments benefit disproportionally more from being firstborn compared to those with lower genetic endowments, providing a clean example of how nature and nurture interact in producing skills. Moreover, since parental investments are a dominant channel driving birth order effects, our results are consistent with dynamic complementarity in skill formation.

Grant Support: NORFACE through the Dynamic of Inequality across the Life Course (DIAL) programme (Grant no. 462-16-100), the National Institute on Aging of the National Institutes of Health under Award R56AG058726, the European Research Council (GEPSI 946647; DONNI 851725).

\section{Scrutinizing the Genetic Relationship Between Psychiatric Disorders}

Mats Nagel ${ }^{1,2}$, Cato Romero ${ }^{1,2}$, Sophie van der Sluis ${ }^{1,2}$

${ }^{1}$ Department of Complex Trait Genetics, Vrije Universiteit Amsterdam, Amsterdam, The Netherlands

${ }^{2}$ Department of Child and Adolescence Psychiatry, section Complex Trait Genetics, Amsterdam UMC, Amsterdam, The Netherlands

Keywords Psychiatric disorder, Local genetic correlation, Genetic overlap

Previous studies have shown that psychiatric disorders (PDs) are highly comorbid and considerably genetically correlated. Including 12 major PDs, this study aimed to scrutinize their genetic overlap as well as their genetic distinctness. SNP-based heritability $\left(h^{2}{ }_{S N P}\right)$ of the 12 PDs ranged between $4.4 \%$ (anxiety) and $36.7 \%$ (obsessivecompulsive disorder; OCD). Genetic correlations $\left(r_{g}\right)$ between the 12 PDs ranged from - 0.13(ADHD-OCD) to 0.83 (depression-anxiety: median $r_{g}=0.24$ ). Using LAVA to estimate local $r_{g}$, we observed 29 significant local $r_{g}$ 's (range -0.84 to 0.98 , median $=0.76$ ), 23 involving schizophrenia, 2 of which were negative, and none were shared between more than 2 traits. Additionally, significant local $r_{g}$ 's were observed between pairs of traits that showed non-significant global $r_{g}$ 's (e.g., insomnia and schizophrenia: global $r_{g}: 0.05 ; 1$ significant opposing local $r_{g}$ 's). Cross-trait meta-analysis on the 12 PDs yielded 222 significant genes, 102 of which overlapped with the 332 genes associated to the 12 individual PDs. Only 39 of 332 genes were associated to two PDs, and three to 3 PDs. Cross-trait meta-analysis yielded 3 biological pathways not observed for any of the 12 individual PDs. Overall, this study shows that while global $r_{g}$ 's between PDs are considerable, local $r_{g}$ 's are unique to certain trait pairs and top genes tend to show little overlap. While low power and low $h^{2}{ }_{S N P}$ play a role here, it may also suggest that the genetic similarity between PDs is driven by a more dispersed signal that does not accumulate in pleiotropic loci but, rather, converges on another level (e.g., biological function).

Grant Support: This work was partially supported by NWO Gravitation program BRAINSCAPES: A Roadmap from Neurogenetics to Neurobiology (NWO: 024.004.012). 


\section{Gene-Environment Interactions in Early Life Cognitive Development}

\author{
Alexandra F. Nancarrow ${ }^{1} \&$ Sophie von Stumm ${ }^{1}$ \\ ${ }^{1}$ Department of Education, University of York, Heslington, York, UK
}

Keywords Gene, Environment, Interaction, Development, Education

Differences in early life cognitive development are widely thought to result from the interplay of genetic and environmental factors, yet no replicable gene-environment interactions $(\mathrm{GxE})$ have been identified. Here, we predicted differences in children's cognitive skills from 2 to 4 years of age by testing interactions between family- and neighborhood-level environments and the genome-wide polygenic score (GPS) for years in education. We addressed 3 research questions: (1) Do GxE effects exist in the prediction on cognitive development? (2) If they exist, to what extent do GxE effects account for children's differences in cognitive development, independent of the main effects of GPS and environmental variables? (3) Which environmental variables, if any, are particularly relevant to the observation of GxE effects? Data came from the Twins Early Development Study (TEDS) in the United Kingdom. Lasso penalized linear regression results revealed a significant main effect of the GPS for years in education on children's general cognitive ability, after controlling for twin age, twin sex, the first ten principal components, and genotyping array, $\beta=$ $.05, S E=.01, p<.05$. Independent of confounders, the GPS for years in education explained .3 percent of the variance in children's general cognitive ability, while environmental measures explained 18.5 percent of the variance. No significant interactions were observed. This research has potential implications for improving children's developmental and educational outcomes in early life.

Grant Support: Nuffield Foundation Grant (EDO/44110)

\section{Exploring Causal Links Between Psychological Distress \& Physical Health}

Zeynep Nas ${ }^{1}$, Helena M. S. Zavos ${ }^{2}$, Athula Sumathipala ${ }^{3,7}$, Kaushalya Jayaweera ${ }^{3}$, Sisira Siribaddana ${ }^{4}$, Matthew Hotopf ${ }^{5,6}$, Frühling V. Rijsdijk ${ }^{1}$

${ }^{1}$ Social, Genetic \& Developmental Psychiatry Centre, Institute of Psychiatry, Psychology and Neuroscience, King's College London, London, UK

${ }^{2}$ Department of Psychology, Institute of Psychiatry, Psychology and Neuroscience, King's College London, London, UK

${ }^{3}$ Institute for Research and Development, Colombo, Sri Lanka

${ }^{4}$ Faculty of Medicine \& Allied Sciences, Rajarata University of Sri Lanka, Anuradhapura, Sri Lanka

${ }^{5}$ Psychological Medicine Department, Institute of Psychiatry, Psychology, and Neuroscience, King's College London, London, UK

${ }^{6}$ NIHR Biomedical Research Centre for Mental Health at the South London and Maudsley NHS Foundation Trust, King's College London, London, UK

${ }^{7}$ Research Institute for Primary Care and Health Sciences, Faculty of Health, Keele University, Keele, UK

Keywords Psychological distress, Physical health, Twin Study, Causation

Psychological distress is associated with poorer physical health, yet the causal relationship between the two domains is not thoroughly understood, especially in non-western populations. Here, we use the direction-of-causation (DOC) twin model to investigate this relationship between psychological distress and physical health using cross-sectional, genetically informative data from the Colombo Twin and Singleton Study (COTASS), as part of the Sri Lankan twin registry. Self-report measures were used to construct out the two latent psychological distress and physical health factors. In this talk, I will present findings from both a bivariate common pathway twin model as well as results obtained from direction of causation twin modelling. Results will be discussed in light of potential limitations, clinical implications, and future directions.

\section{Modeling Assortative Mating and Other Selection with OpenMx}

${ }^{1}$ Michael C. Neale and ${ }^{2}$ Joshua N. Pritikin

${ }^{1}$ Departments of Psychiatry, Virginia Institute for Psychiatric and Behavioral Genetics, Virginia Commonwealth University, Richmond, Virginia, USA.

${ }^{2}$ Human \& Molecular Genetics, Virginia Institute for Psychiatric and Behavioral Genetics, Virginia Commonwealth University, Richmond, Virginia, USA.

Keywords Assortative mating, Structural equation modeling, Multivariate analysis, Selection

Resemblance between spouses has numerous potential sources, including: direct phenotypic homogamy, population stratification and marital interaction. Even within phenotypic assortment, spouse resemblance for one trait may be due to mate selection on a correlated trait. Multivariate modeling of spousal resemblance can help to distinguish between such primary and secondary mechanisms. To date, modeling these alternative mechanisms has proved challenging, especially when they are considered jointly. Recent developments to OpenMx software have facilitated the specification of such models in two ways. First, Pearson-Aitken selection matrix functions that calculate the covariances of all measured variables following selection on a subset of them are useful for matrix-based analyses. Second, and apparently a first for structural equation modeling software, it is possible to use co-paths, represented by lines with no arrowheads, to represent direct phenotypic homogamy. In the multivariate context, homogamy for some variables may precede that for others. Accordingly, it is possible to specify the order in which the assortment for different variables occurs. For substance use, marital resemblance is substantial.

Grant Support: NIH/NIDA funding 1R01DA049867

\section{Genetic and Environmental Influences on Sleep-Wake Behaviors in Adolescence}

Victoria S. O'Callaghan ${ }^{1}$, Narelle K. Hansell ${ }^{1}$, Wei Guo ${ }^{2}$, Joanne Carpenter ${ }^{3}$, Haochang Shou ${ }^{4,5}$, Lachlan T. Strike ${ }^{1}$, Kerrie McAloney ${ }^{8}$, Katie L. McMahon ${ }^{6}$, Enda M. Byrne ${ }^{7}$, Nicholas G. Martin ${ }^{8}$, Ian B. Hickie ${ }^{3}$, Kathleen Merikangas ${ }^{4}$, Margaret J. Wright ${ }^{1,9}$

${ }^{1}$ Queensland Brain Institute, University of Queensland, Brisbane, Australia

${ }^{2}$ Statistical Genomics and Data Analysis Core, National Institute of Mental Health, National Institutes of Health, Bethesda, MD USA

${ }^{3}$ Brain and Mind Institute, University of Sydney, Sydney, NSW, Australia 
${ }^{4}$ Genetic Epidemiology Branch, National Institute of Mental Health, National Institutes of Health Bethesda, MD, USA

${ }^{5}$ Department of Biostatistics, Epidemiology, and Informatics, University of Pennsylvania Perelman School of Medicine, Philadelphia, PA, USA

${ }^{6}$ Queensland University of Technology, Brisbane, Australia

${ }^{7}$ Institute of Molecular Bioscience, University of Queensland, Brisbane, Australia

${ }^{8}$ QIMR Berghofer Medical Research Institute, Brisbane, QLD 4066, Australia

${ }^{9}$ Centre for Advanced Imaging, University of Queensland, Brisbane, Australia

Keywords Sleep, Adolescence, Heritability, Twins, Genetics, Actigraphy

The study objective was to investigate the role of genetic and environmental factors on sleep-wake behaviors across adolescence and differences between school and non-school nights. Four hundred and ninety-five participants (aged 9 to $18 ; 55 \%$ females), including 93 monozygotic (MZ) and 117 dizygotic (DZ) twin pairs, and 75 unmatched twins, wore a wrist activity monitor and completed a sleep diary for two weeks. Individual differences in sleep onset, wake time, and sleep midpoint were similarly influenced by additive genetic $(50 \% ; 48 \% ; 44 \%$ of total variance) and shared environmental $(36 \%$; $31 \% ; 42 \%$ ) factors, with a predominant genetic influence for sleep duration $(62 \%)$ and restorative sleep $(43 \%)$. When we stratified by age, in older adolescents (aged 16-17), genes accounted for more of the variance than in younger adolescents (aged 9-14), and shared environmental factors were no longer significant. Bivariate analyses showed sleep duration and sleep midpoint were correlated $(r \mathrm{p}=-$ $.43, r \mathrm{G}=.54$ ), due to a common genetic source. Additionally, while heritability was similar for school and non-school nights, there were genetic sources common and specific to each night type for all objectively measured sleep-wake behaviors. Across adolescence, there is a strong genetic influence on sleep phenotypes, but genes have a greater impact on older adolescents' sleep timing than younger adolescents, likely to be influenced by family environment. The relationship between sleep maintenance and chronotype is entirely due to a common genetic source. Both common and specific genetic sources influence sleep-wake behaviors on school and non-school nights in adolescence.

\section{Multi-omics Prediction of Complex Traits and Exposers: Polygenic and DNA Methylation Scores}

Veronika V. Odintsova ${ }^{1}$, Jenny van Dongen ${ }^{1}$, Dorret I. Boomsma ${ }^{1}$, NTR (Netherlands Twin Register), BBMRI-BIOS (Biobank-based integrative omics study), ALSPAC (Avon Longitudinal Study of Parents and Children), ACTION (Aggression in Children: Unraveling gene-environment interplay to inform Treatment and InterventiON strategies) consortium

${ }^{1}$ Department of Biological Psychology, Amsterdam Public Health Research Institute, Vrije Universiteit Amsterdam, Amsterdam, The Netherlands

Keywords DNA methylation scores, Polygenic scores, Multi-omics prediction

DNA-based predictors of complex traits and exposures can serve to improve prediction of health and behavioral outcomes, to optimize risk stratification, and are increasingly considered for application in social sciences and education. Whereas DNA-based predictors are static, other predictors such as those based on epigenome data are dynamic and may capture both genetic and environmental information. We will discuss prediction by polygenic scores and DNA methylation scores created using weights derived from summary statistics of large genome- and epigenome-wide studies, for several traits (birth weight, BMI, left-handedness, and aggression) and exposures (prenatal maternal smoking and smoking). Predictive ability of the scores in multi-omics prediction models was evaluated on the Netherlands Twin Register cohorts of adults and children with DNA methylation in blood and buccal cells. We examined if the DNA methylation scores add predictive value above the polygenic scores for DNA methylation data obtained from different tissues and ages. Our studies illustrate the value of combining polygenic scores with information from methylation data for complex traits and exposure prediction.

Grant Support: the Netherlands Organization for Scientific Research (NWO): Biobanking and Biomolecular Research Infrastructure (BBMRI-NL, 184.033.111); the BBMRI-NL funded BIOS Consortium (NWO184.021.007); "Aggression in Children: Unraveling geneenvironment interplay to inform Treatment and InterventiON strategies" project (ACTION) funding from the European Union Seventh Framework Program (FP7/2007-2013) under grant agreement no 602768; NWO 480-15-001/674: Netherlands Twin Registry Repository: researching the interplay between genome and environment, the Avera Institute for Human Genetics and by multiple grants from the Netherlands; NWO/SPI 56-464-14192, Genetic Association Information Network (GAIN) of the Foundation for the National Institutes of Health, Rutgers University Cell and DNA Repository (NIMH U24 MH068457-06), the National Institutes of Health (NIH R01 HD042157-01A1, MH081802, Grand Opportunity grants 1RC2 MH089951 and 1RC2 MH089995) and European Research Council (ERC-230374); NWO Large Scale infrastructures, X-Omics (184.034.019); the Royal Netherlands Academy of Science Professor Award (PAH/6635).

\section{Causal Influences of Same-Sex Attraction on Psychological Distress and Risky Sexual Behaviors: Evidence for Bidirectional Effects Using the Mendelian Randomization-Direction of Causation Model}

Olakunle Ayokunmi Oginni ${ }^{1}$, Kai Xiang Lim $^{1}$, Kirstin Purves ${ }^{1}$, Patrick Jern ${ }^{2}$, Frühling Vesta Rijsdijk ${ }^{1}$

${ }^{1}$ The Social, Genetic and Developmental Psychiatry centre, Institute of Psychiatry, Psychology and Neuroscience, Denmark Hill, King's College London

${ }^{2}$ Department of Psychology, Åbo Akademi University, Åbo, Finland

Keywords Same-sex attraction, Psychological distress, Risky sexual behavior, Mendelian Randomization, Direction of Causation

Although minority stress explains health disparities among same-sex attracted compared to heterosexual individuals; there is limited evidence to infer causation. This study determined whether same-sex attraction was causally associated with psychological distress and risky sexual behavior. The sample comprised monozygotic and dizygotic twins, and non-twin siblings $(\mathrm{n}=2036,3780$ and 2356 respectively) genotyped and assessed for same-sex attraction, psychological distress, and risky sexual behavior. Causal influences were investigated with same-sex attraction as the predictor and psychological distress and risky sexual behavior as the outcomes in two separate Mendelian Randomization-Direction of Causation (MRDoC) models using OpenMx in R. Supplementary analyses tested reverse 
causal influences from psychological distress and risky sexual behavior towards same-sex attraction in separate MRDoC models, and moderation of all causal paths by early-life adversities. There were significant causal influences flowing from same-sex attraction to psychological distress and risky sexual behavior (standardized coefficients $=0.13$ and $0.16 ; 95 \%$ CIs $0.03,0.23$ and $0.08,0.25$ respectively). Supplementary analyses further indicated causal influences flowing from psychological distress and risky sexual behavior towards same-sex attraction. Exploratory analyses indicated no significant moderation of causal paths by early-life adversities. Causal influences from same-sex attraction to psychological distress and risky sexual behavior may reflect minority stress which reinforces ongoing measures to minimize social disparities. Causal influences flowing in the opposite direction may reflect rejection sensitivity, stigma-inducing outcomes of risky sexual behavior and recall bias; however, further research is required to specifically investigate these processes.

\section{Polygenic Prediction Within and Between Families from a 3-Million-Person GWAS of Educational Attainment}

Aysu Okbay ${ }^{1}$, Yeda $\mathrm{Wu}^{2}$, Nancy Wang ${ }^{3}$, Hariharan Jayashankar ${ }^{3}$, Michael Bennett ${ }^{3}$, Seyed Moeen Nehzati ${ }^{4}$, Julia Sidorenko ${ }^{2}$, Hermon Kweon ${ }^{1}$, Grant Goldman ${ }^{3}$, Tamara Gjorgjieva ${ }^{3}$, Yunxuan Jiang ${ }^{5}$, Chao Tian ${ }^{5}$, Rafael Ahlskog ${ }^{6}$,

Patrik K.E. Magnusson ${ }^{7}$, Sven Oskarsson ${ }^{6}$, Caroline Hayward ${ }^{8}$, Archie Campbel1 ${ }^{9,10}$, David Porteous ${ }^{10,11}$, Jeremy Freese ${ }^{12}$, Pamela Herd ${ }^{13}$, 23andMe Research Team ${ }^{14}$, Social Science Genetic Association Consortium ${ }^{14}$, Chelsea Watson ${ }^{4}$, Jonathan Jala ${ }^{4}$,

Dalton C. Conley ${ }^{15}$, Philipp D. Koellinger ${ }^{1,16}$,

Magnus Johannesson ${ }^{17}$, David I. Laibson ${ }^{18}$, Michelle N. Meyer ${ }^{19}$, James J. Lee ${ }^{20}$, Augustine Kong ${ }^{21}$, Loic Yengo ${ }^{2}$,

David Cesarini ${ }^{3,22,23}$, Patrick Turley ${ }^{24,25}$, Peter M. Visscher ${ }^{2}$,

Jonathan P. Beauchamp ${ }^{26}$, Daniel J. Benjamin ${ }^{3,4,27}$,

and Alexander I. Young ${ }^{4}$

${ }^{1}$ Department of Economics, School of Business and Economics, Vrije Universiteit Amsterdam, Amsterdam, the Netherlands

${ }^{2}$ Institute for Molecular Bioscience, University of Queensland, Brisbane, Australia

${ }^{3}$ National Bureau of Economic Research, Cambridge, MA, USA

${ }^{4}$ UCLA Anderson School of Management, Los Angeles, CA, USA

${ }^{5} 23$ andMe, Inc., Mountain View, CA, USA

${ }^{6}$ Department of Government, Uppsala University, Uppsala, Sweden

${ }^{7}$ Swedish Twin Registry, Department of Medical Epidemiology and Biostatistics, Karolinska Institutet, Stockholm, Sweden

${ }^{8}$ MRC Human Genetics Unit, Institute of Genetics and Molecular Medicine, University of Edinburgh, Western General Hospital, Edinburgh, United Kingdom

${ }^{9}$ Centre for Genomic and Experimental Medicine, Institute of Genetics \& Molecular Medicine, University of Edinburgh, Western General Hospital, Edinburgh, United Kingdom

${ }^{10}$ Usher Institute, University of Edinburgh, Edinburgh, United Kingdom

${ }^{11}$ Centre for Cognitive Ageing and Cognitive Epidemiology, University of Edinburgh, Edinburgh, United Kingdom

${ }^{12}$ Department of Sociology, Stanford University, Stanford, CA, USA

${ }^{13}$ McCourt School of Public Policy, Georgetown University, Washington, DC, USA

${ }^{14} \mathrm{~A}$ full list of members and affiliations appears at the end of the paper

${ }^{15}$ Department of Sociology, Princeton University, Princeton, NJ, USA
${ }^{16}$ Robert M. La Follette School of Public Affairs, University of Wisconsin-Madison, Madison, WI, USA

${ }^{17}$ Department of Economics, Stockholm School of Economics, Stockholm, Sweden

${ }^{18}$ Department of Economics, Harvard University, Cambridge, MA, USA

${ }^{19}$ Center for Translational Bioethics and Health Care Policy, Geisinger Health System, Danville, PA, USA

${ }^{20}$ Department of Psychology, University of Minnesota Twin Cities, Minneapolis, MN, USA

${ }^{21}$ Big Data Institute, Li Ka Shing Centre for Health Information and Discovery, University of Oxford, United Kingdom

${ }^{22}$ Department of Economics, New York University, New York, NY, USA

${ }^{23}$ Center for Experimental Social Science, New York University, New York, NY, USA

${ }^{24}$ Department of Economics, University of Southern California, Los Angeles, CA, USA

${ }^{25}$ Center for Economic and Social Research, University of Southern California, Los Angeles, CA, USA

${ }^{26}$ Interdisciplinary Center for Economic Science and Department of Economics, George Mason University, Fairfax, VA, USA

${ }^{27}$ Human Genetics Department, UCLA David Geffen School of Medicine, Los Angeles, CA, USA

Keywords Educational attainment, GWAS, Direct/indirect effects, Dominance, Polygenic score

Educational attainment (EA) is an important dimension of socioeconomic status that features prominently in research by social scientists, epidemiologists, and other medical researchers. We conduct a GWAS of EA in a sample of 3,037,499 individuals and identify 3952 approximately uncorrelated genome-wide-significant SNPs. We also conduct a GWAS of the $\mathrm{X}$ chromosome $(N=2,713,033)$ and the first large-scale GWAS of dominance variance of EA on the autosomes $(N=2,574,253)$. Our X-chromosome GWAS identifies 57 approximately uncorrelated genome-wide-significant SNPs. Our dominance GWAS identifies no genome-wide-significant SNPs; moreover, with high confidence, we can rule out the existence of any common SNPs whose dominance effects explain more than a negligible fraction of the variance in EA. We report evidence of directional dominance for EA. We also investigate the scope and sources of the predictive power of a genome-wide polygenic predictor, or polygenic index (PGI), of EA. The PGI explains $12-16 \%$ of the variation in EA and adds nontrivial predictive power for 10 diseases we examine, even after controlling for disease-specific PGIs. Using a combined sample of $\sim 53,000$ individuals with genotyped siblings and $\sim 3500$ individuals with both parents genotyped, we examine the predictive power of the EA PGI controlling for parental EA PGIs. The PGI's associations with EA and other phenotypes falls by roughly half when controlling for parental PGIs. Finally, we use the EA and other PGIs to study assortative mating and find that the correlation between spouses' EA PGIs is far too large to be consistent with phenotypic assortment alone.

Grant Support: The study was supported by funding from the Ragnar Söderberg Foundation (E42/15, D.C.);, the Swedish Research Council (421-2013-1061, M.J.; 2019-00244, S.O.);, an ERC Consolidator Grant (647648 EdGe, P.K.); the Pershing Square Fund of the Foundations of Human Behavior (D.L.);, Open Philanthropy (01062300001, D.J.B., P.T., M.N.M.);, Riksbankens Jubileumsfond P180782:1 (S.O.);, Netherlands Organisation for Scientific Research VENI grant 016.Veni.198.058 (A.O.); , and the NIA/NIH through grants R24-AG065184 (D.J.B.) and R01-AG042568 (D.J.B.) to the University of California Los Angeles, ; K99-AG062787-01 (P.T.) to Massachusetts General Hospital; the NIA/NIMH through grants 
1R01-MH101244-02 (P.T.; PI: Benjamin M. Neale) and 5U01MH109539-02 (P.T.; PI: B.M.N.) to the Broad Institute at Harvard and MIT; the Government of Canada through Genome Canada and the Ontario Genomics Institute (OGI-152) (J.P.B.); the Social Sciences and Humanities Research Council of Canada (J.P.B.); the National Health and Medical Research Council through grant GNT113400 (P.M.V.); and the Australian Research Council (P.M.V.).

\section{Shared Environment in Second Language Learning}

\author{
Ordoñana, $\mathrm{C}^{1}$; Madrid-Valero, $\mathrm{JJ}^{2}$; Sánchez-Romera, $\mathrm{JF}^{3,4}$; \\ Ordoñana, $\mathrm{JR}^{3,4}$ \\ ${ }^{1}$ Department of English, University of Murcia \\ ${ }^{2}$ Department of Health Psychology, University of Alicante \\ ${ }^{3}$ Department of Human Anatomy and Psychobiology, University of \\ Murcia \\ ${ }^{4}$ Murcia Instituto of Biomedical Research, IMIB-Arrixaca, Murcia, \\ Spain
}

Keywords Heritability, Second language, Education

Learning a second language has a decisive role on educational progress, autonomy, and self-development in the globalized word. Surprisingly, despite the interest of this phenotype, there is much to know about why individuals differ substantially in their second language performance. The objective of this work is to analyze the relative contribution of genetic and environmental factors to the acquisition of a second (foreign) language in our educational context. Results from the standard exam of second language for university access were analyzed in 98 pairs of twins ( $52 \mathrm{MZ}$; $46 \mathrm{DZ}$ ) participants in the Murcia Twin Registry (76.5\% women). 94.3\% were English exams and the rest French. Mean score was 6.1 (SD 2.2). A univariate model was applied using structural equation models to estimate heritability. Correlation was higher for $\mathrm{MZ}$ twins $(\mathrm{rMZ}=.71 ; 95 \% \mathrm{CI}$ $.53, .83)$ than for $\mathrm{DZ}(\mathrm{rDZ}=.59 ; 95 \%$ CI $.34, .76)$. An ACE model where family factors have a substantial effect showed a good fit to the data $(\mathrm{A}=.32 ; 95 \% \mathrm{CI} .00, .79 ; \mathrm{C}=.41 ; 95 \% \mathrm{CI} .00, .72 ; \mathrm{E}=.28 ; 95 \%$ $\mathrm{CI} .18, .44)$. AE and CE models did not differ significantly from the full ACE model but both of them with a similar fit $(p=.09$ and $p=$ .13 , respectively). Shared environmental factors explain a greater proportion of variance in this phenotype than genetic or unique environmental ones. The weight of the shared environment and, therefore, heritability may vary between countries depending on socio-cultural circumstances.

Grant Support: Ministerio de Ciencia, Innovación y Universidades Spain (RTI2018-095185-B-I00) co-funded by European Regional Development Fund (FEDER)

\section{Large-Scale Deep Sequencing Meta-analysis of Alcohol and Tobacco Use: Effects of Rare Variation}

Jacqueline M. Otto ${ }^{1}$, on behalf of the GWAS \& Sequencing Consortium of Alcohol and Nicotine Use and the Trans-Omics for Precision Medicine program

${ }^{1}$ Department of Psychology, University of Minnesota, Minneapolis, MN, USA

Keywords Rare variation, Meta-analysis, Whole-genome Sequencing, Gene-based analysis, Substance use
Alcohol and tobacco use are moderately heritable behaviors $\left(\mathrm{h}^{2} \approx\right.$ 0.50 ), and genome-wide association studies (GWAS) have identified a large number of common variants, each with small effect and uncertain direct causality. Much of the remaining missing heritability for complex traits is thought to be attributable to rare variation (minor allele frequency $[\mathrm{MAF}]<0.01$ ). However, such investigations using deep whole-genome sequencing (WGS) require large samples and have not yet been conducted for substance use and dependence. Therefore, the current study sought to test whether rare variants were associated with measures of alcohol and tobacco use and addiction. Study phenotypes included a measure of drinks consumed per week, as well as smoking initiation, age of smoking initiation, cigarettes smoked per day, and smoking cessation. Deep whole-genome sequences were obtained for $\sim 155,000$ samples as part of the TransOmics for Precision Medicine (TOPMed) consortium, and this dataset was used as a reference panel to impute genotypes for $\sim 500,000$ individuals in UK Biobank, a large-scale richly-phenotyped biomedical and research database. Whole-exome sequences (WES) were also obtained for $\sim 200,000$ UK Biobank participants. Planned analyses are ongoing at this time, and include a rare variant metaanalysis of TOPMed WGS, UK Biobank genome-wide array data imputed to TOPMed, and UK Biobank WES, as well as rare-variant gene-based tests. This study will allow for well-powered discovery of rare variants and genes associated with alcohol and tobacco use and inform our understanding of their biological etiology.

Grant Support: NIDA R01DA044283

\section{Genetic and Environmental Moderation of Cognitive Performance by Smoking Behavior}

Shandell Pahlen ${ }^{1}$, Carol E. Franz ${ }^{2}$, William S. Kremen ${ }^{2}$,

Anna K. Dahl Aslan ${ }^{3,4,5}$, Teresa Lee ${ }^{6}$, Perminder Sachdev ${ }^{6,7}$,

Vibeke S. Catts ${ }^{6}$, \& Chandra A. Reynolds ${ }^{1}$, for the IGEMS Consortium

${ }^{1}$ Department of Psychology, University of California, Riverside, Riverside, California, USA

${ }^{2}$ Department of Psychiatry, University of California, San Diego, San Diego, California, USA

${ }^{3}$ School of Health Sciences, University of Skövde, Skövde, Sweden ${ }^{4}$ Department of Medical Epidemiology and Biostatistics, Karolinska Institutet, Stockholm, Sweden

${ }^{5}$ Institute of Gerontology and Aging Research Network - Jönköping (ARN-J), School of Health and Welfare, Jönköping University, Jönköping, Sweden

${ }^{6}$ Centre for Healthy Brain Ageing, School of Psychiatry, University of New South Wales Sydney, Sydney, NSW, Australia

${ }^{7}$ Neuropsychiatric Institute, The Prince of Wales Hospital, UNSW, Randwick, NSW, Australia

Keywords Smoking Behavior, Cognitive Functioning, GxE Moderation

Tobacco use is a known health burden and phenotypic research has found worse cognitive functioning linked with smoking behavior. To further examine the etiological impact of smoking behavior on cognitive functioning biometric moderation models were tested using twin data from 12 studies in the Interplay of Genes and Environment Across Multiple Studies (IGEMS) consortium ( $\mathrm{N}=24,536[\mathrm{~F}=$ $51.3 \%$, Mage $=60.7$ years $[\mathrm{SD}=12.2]$, Complete pairs based on cognitive task: 476 to $2250 \mathrm{MZ}$ and 691 to $4201 \mathrm{DZ}$ ). Smoking measures included smoking status variables (e.g., ever and current smokers) and $\log 10$ transformed pack-years. Cognitive measures 
included fluency, processing speed, and spatial reasoning. Generalized estimating equation models, controlling for pair dependency, age, sex, and country showed current smokers had significantly lower scores on all cognitive measures (Cohen's d: -.11 [fluency] to -.35 [spatial]); pack-years showed similar patterns (Cohen's d: - .04 [fluency] to -.14 [speed] per $\log _{10}$ year). Extended univariate moderation models were tested entering smoking status and pack-years. Likelihood ratio tests supported significant moderation by smoking status for speed and pack-years for fluency, resulting in lower genetic and higher environmental influences with increasing pack-years. Processing speed differed with higher genetic contributions for smokers. Spatial reasoning followed fluency trends but was non-significant. Overall, moderation patterns suggest greater smoking-related environmental influences across all tasks. Smoking behavior represents a complex moderator of the etiology of age-sensitive cognitive tasks. Further work examining additional moderating contexts (e.g., sex, country, birthyear) will be reviewed.

Grant Support: R01 AG059329, R01 AG060470, R01 AG050595, R01 AG022381

\section{Childhood Externalizing Problems: The Indirect Influence of Parental Negative Talk and Shared Genetic Effects of Child Aggression}

Emily C. Pali ${ }^{1}$, M.A., Matthew R. Jamnik ${ }^{1}$, M.A., Riley L. Marshall, M.S. ${ }^{1}$, Rachel L. Weisbecker, B.A. ${ }^{1}$, Cheyenne Vazquez, B.S. ${ }^{2}$, Lisabeth F. DiLalla, Ph.D. ${ }^{2}$

${ }^{1}$ School of Psychological \& Behavioral Sciences, Southern Illinois University, Carbondale, IL, USA

${ }^{2}$ Department of Family \& Community Medicine, Southern Illinois University School of Medicine, Carbondale, IL, USA

\section{Keywords Aggression, Preschool, Externalizing, Negative talk}

Externalizing behaviors can cause serious, costly, and long-standing problems for children. Parent-child interaction therapy (PCIT) is aimed at combating childhood externalizing problems in children aged 2-7. PCIT aims to reduce disruptive behavior through decreasing negative talk (NTA) and other negative parenting behaviors while increasing positive parenting. In this study, we hypothesized (1) parent NTA and childhood aggression would be associated with children's externalizing behavior, and (2) these would share genetic variance. As part of a longitudinal twin study (DiLalla and Jamnik, 2019) of 328 twins/triplets, 4-year-old children and one parent worked together on a puzzle task. Reliable coders later rated parents for NTA and children for aggressive behaviors. At age 5, parents rated children's externalizing behaviors. Mediation analyses showed that parental NTA impacted child externalizing indirectly via child aggressive behaviors. Multilevel modeling examined within and between twin/family variance to assess causality and shared genetic effects. NTA was causally related to externalizing; the twin who experienced more NTA had higher externalizing behaviors one year later, $\mathrm{p}=.018$. There was no shared genetic variance between these measures. However, there was significant shared genetic variance between age 4 aggression and age 5 externalizing behaviors, $\mathrm{p}=.005$. Thus, NTA has an environmental influence on externalizing, whereas aggression's influence is largely genetic. This could have implications for clinical treatment focused on the impact of parental NTA on children's behaviors.

\section{References}

${ }^{1}$ DiLalla LF, Jamnik MR (2019) The Southern Illinois Twins/Triplets and Siblings Study (SITSS): A longitudinal study of early child development. Twin Research and Human Genetics, 22(6), 779-782.

Grant Support: Funding was provided by a grant from the Central Research Committee of SIU School of Medicine to LFD.

\section{Genetic and Environmental Moderation Among Plasma-Based Biological Markers of Alzheimer's Disease}

Matthew S. Panizzon ${ }^{1,2}$; Robert A. Rissman ${ }^{3}$, Jeremy A. Elman ${ }^{1,2}$; Nathan Gillespie ${ }^{4}$; Michael C. Neale ${ }^{4}$; Chandra Reynolds ${ }^{5}$; Michael Lyons $^{6}$; Carol E. Franz ${ }^{1,2}$; and William S. Kremen ${ }^{1,2}$

${ }^{1}$ Department of Psychiatry, University of California San Diego

${ }^{2}$ Center for Behavior Genetics of Aging, University of California San Diego

${ }^{3}$ Department of Neuroscience, University of California San Diego

${ }^{4}$ Virginia Institute for Psychiatric and Behavioral Genetics

${ }^{5}$ Department of Psychology, University of California Riverside

${ }^{6}$ Department of Psychological and Brain Sciences, Boston University

Keywords Beta amyloid, tau, Neurodegeneration, Genetic moderation, Alzheimer's disease

The current research framework for Alzheimer's disease (AD) defines the condition in terms of the progressive accumulation of beta-amyloid $(\mathrm{A} \beta)$ and tau, as well as progressive neurodegeneration. This framework represents a biological definition of $\mathrm{AD}$, and shifts $\mathrm{AD}$ research toward examining younger cohorts where the progression of biological markers can be examined prior to dementia onset. The genetic analysis of these biomarkers is still in its infancy, as little is known regarding their genetic and environmental determinants, or if the magnitude of genetic and environmental influences change over disease progression. The goal of the present study was to determine whether plasma-based $\mathrm{A} \beta$, tau, and neurodegeneration-assessed by neurofilament light (NFL)—moderated the genetic and environmental determinants of one another. Data were from the Vietnam Era Twin Study of Aging. The average age at assessment was 67.1 years $(\mathrm{SD}=2.6)$. Using a bivariate model for the analysis of gene-environment interaction, we found evidence for significant genetic and environmental moderation across multiple biomarkers. All variance components of $A \beta_{42}$, for example, significantly increased as a function of NFL level, resulting in a change in heritability from .26 to .47 . A similar pattern of moderation was observed between tau and $A \beta_{42}$. The heritability of $A \beta_{42}$ also increased as a function of $A \beta_{40}$, a more abundant form of the peptide, while the genetic correlation between them decreased. These results suggest that the degree to which AD biomarkers are influenced by genetic and environmental factors is in part dependent on disease staging as indexed by biomarker accumulation.

Grant Support: R01 AG050595, R01 AG023381, R01 AG056410, P01 AG055367, and K01 AG063805 


\section{Dynamix Transactions Between Externalizing Behaviors and Inconsistent Discipline}

Stephane Paquin ${ }^{1}$, Jody M. Ganiban ${ }^{2}$, Daniel S. Shaw ${ }^{3}$, David Reiss ${ }^{4}$, Leslie D. Leve ${ }^{5}$, Misaki N. Natsuaki ${ }^{6}$, Jenae M. Neiderhiser ${ }^{1}$

${ }^{1}$ Department of Psychology, The Pennsylvania State University, University Park, PA, USA

${ }^{2}$ Department of Psychological and Brain Sciences, George Washington University, Washington, DC, USA

${ }^{3}$ Department of Psychology, University of Pittsburgh, Pittsburgh, PA, USA

${ }^{4}$ Yale Child Study Center, Yale University, New Haven, CT, USA

${ }^{5}$ College of Education, University of Oregon, Eugene, OR, USA

${ }^{6}$ Department of Psychology, University of California at Riverside, Riverside, CA, USA

Keywords Externalizing behaviors, Inconsistent discipline, Latent change score, Adoption design, Child development

The developmental course of child externalizing behaviors is unlikely to be independent as reciprocal effects have been shown with negative parenting. We apply a bivariate latent change score model to test reciprocal effects between child's externalizing behavior and parent's inconsistent discipline and intraindividual change of these behaviors. Using a parent-offspring adoption design, we also test for the presence of evocative $r \mathrm{GE}$ on parent's inconsistent discipline practices.

This study uses data from 561 children from the Early Growth and Development Study. Parents reported on their use of inconsistent discipline practices and on their children's externalizing behaviors during childhood and early adolescence $(4.5,6,7$ and 11 years of age). Preliminary results from the best fitting bivariate model indicate that only inconsistent discipline was associated with future change in externalizing behaviors. This model showed that children's externalizing behaviors follow a mean decline (growth rate $b=-11.720$ ) that got steeper as they displayed more externalizing behaviors (proportional change related to past externalizing $b=-.476$ ), but that mean decline was dampened by their parents' inconsistent discipline practices (proportional change related to past inconsistent discipline $b$ $=7.035$ ). Birth mother's externalizing behaviors was examined as a predictor of adoptive parents' inconsistent discipline. No evidence of evocative $r$ GE was found. In summary, our findings control for both passive and evocative $r \mathrm{GE}$ and thus, indicate that parents' inconsistent discipline may have a mostly environmental effect and prevent a decline in children's externalizing behaviors during childhood and early adolescence.

Grant Support: Fond de Recherche du Québec - Santé; R01HD042 608; R01DA020585; R01MH092118; R01DK090264; R01DA03 5062; UH3OD023389; R01DA045108.

\section{Exploring Relationships Between Internalizing Problems and Risky Sexual Behavior: A Twin Study}

Katie N. Paulich ${ }^{1,2}$, Michael C. Stallings ${ }^{1,2}$, John K. Hewitt ${ }^{1,2}$ ${ }^{1}$ Institute for Behavioral Genetics, University of Colorado Boulder, Boulder, CO, USA

${ }^{2}$ Department of Psychology and Neuroscience, University of Colorado Boulder, Boulder, CO, USA

Keywords Risky sexual behavior, Internalizing problems, Twin study, Number of lifetime partners

Previous research has linked risky sexual behavior (RSB) to externalizing problems (Ross et al., 2019; Clark et al, 2021) and to substance use (Huibregtse et al., 2020), but little research has been conducted on relationships between RSB and internalizing problems. Component measures of RSB-e.g., number of lifetime sexual partners and age at first sexual encounter-demonstrate heritability, as do internalizing problems. The current study examines relationships between RSB and internalizing problems, utilizing data from twins participating in the Center on Antisocial Drug Dependence. We first explore genetic and environmental influences on both number of lifetime sexual partners and age at first sexual encounter, as well as on depression, neuroticism, and self-esteem. We then examine relationships between RSB and internalizing problems via confirmatory factor analysis and bivariate Cholesky decomposition. Our research is relevant to understanding links between risky sexual behavior and mental health.

\section{References}

Clark, D.A., Durbin, C.E., Heitzeg, M.M., Iacono, W.G., McGue, M., Hicks, B.M. (2021) Adolescent sexual development and peer groups: Reciprocal associations and shared genetic and environmental influences. Arch Sex Behav., 50(1), 141-160.

Huibregtse, B., Hatoum, A.S., Corley, R.P., Rhea, S.A., Hewitt, J.K., \& Stallings, M.C. (2020). Etiological Overlap Between Sex Under the Influence and Number of Lifetime Sexual Partners. Behavior Genetics, 1-18.

Ross, J. M., Granja, K., Duperrouzel, J.C., Pacheco-Colón, I., Lopez-Quintero, C., Hawes, S. W., \& Gonzalez, R. (2019). Risky sexual behavior among adolescents: The role of decision-making, problems from cannabis use and externalizing disorder symptoms. Journal of Clinical and Experimental Neuropsychology, $41: 3,300-311$.

\section{Grant Support: T32 MH016880, P50 DA011015}

\section{Genetic and Early Environmental Predictors of Retrospective Self-reports of Childhood Trauma}

Alicia J. Peel ${ }^{1}$, Jessie R. Baldwin ${ }^{1,2}$, Gerome Breen ${ }^{1,3}$, Jonathan R.I. Coleman ${ }^{1,3}$, Jean-Baptiste Pingault ${ }^{1,2}$, Kirstin L. Purves ${ }^{1}$, Megan Skelton ${ }^{1,3}$, Abigail ter Kuile ${ }^{1,3}$, Andrea Danese $^{1,4,5}$ \& Thalia C. Eley ${ }^{1,3}$

${ }^{1}$ Social, Genetic and Developmental Psychiatry Centre; Institute of Psychiatry, Psychology \& Neuroscience; King's College London, London, United Kingdom

${ }^{2}$ Division of Psychology and Language Sciences, Department of Clinical, Educational and Health Psychology, University College London, London, United Kingdom

${ }^{3}$ UK National Institute for Health Research (NIHR) Biomedical Research Centre, South London and Maudsley NHS Trust, London, United Kingdom

${ }^{4}$ Department of Child \& Adolescent Psychiatry, Institute of Psychiatry, Psychology \& Neuroscience, King's College London, London, SE5 8AF, United Kingdom

${ }^{5}$ National and Specialist CAMHS Trauma, Anxiety, and Depression Clinic, South London and Maudsley NHS Foundation Trust, London, SE5 8AF, United Kingdom

Keywords Self-report, Trauma, Polygenic scores

Background. Evidence suggests that retrospective self-reports of childhood trauma are associated with a greater risk of psychopathology in adulthood than are prospective measures. However, it remains unclear why retrospectively self-reported trauma confers a 
greater risk for poor mental health outcomes. Investigating the heritable characteristics and environmental adversities associated with measures of self-reported trauma could increase understanding of this risk pathway to psychopathology. Methods. Our sample included 3,963 unrelated individuals from the Twins Early Development Study. We tested whether polygenic scores for 21 psychiatric, cognitive, anthropometric and personality traits were associated with childhood trauma retrospectively self-reported in adulthood and whether these associations remained after controlling for composite scores of early environmental adversity between birth and age 16, using linear regression models. Results. Retrospectively self-reported childhood trauma was positively associated with polygenic scores for autism spectrum disorder (ASD), body mass index and risky behaviours. When composite scores of environmental adversity were included in the model, only the association with the polygenic score for ASD remained significant. Conclusion. Retrospective self-reports of childhood trauma are associated with heritable characteristics of the reporter. Genetic liability to ASD may increase sensitivity to experiencing or interpreting events as traumatic. Associations between genetic predisposition for risky behaviour and high BMI with self-reported childhood trauma may be environmentally-mediated. Studies of the association between self-reported childhood trauma and later life outcomes should consider that genetically-influenced reporter characteristics may confound associations, both directly and through gene-environment correlation.

\section{Well-Being is More than "A Personality Thing": Common and Unique Genetic Effects on Higher Order Factors of Personality and Well-Being}

\author{
Dirk H.M. Pelt ${ }^{1,2}$, \& Meike Bartels ${ }^{1,2}$ \\ ${ }^{1}$ Department of Biological Psychology, Vrije Universiteit Amster- \\ dam, The Netherlands \\ ${ }^{2}$ Amsterdam Public Health Research Institute, Amsterdam, The \\ Netherlands
}

Keywords Personality, Well-being, Extended twin design, Higher order factors

Based on studies finding that genetic effects on well-being were completely shared with personality, some authors have concluded that "wellbeing is a personality thing" (Weiss et al. 2008). However, in these studies, well-being is often measured by specific traits (e.g., life satisfaction), while recent studies have suggested that well-being covers a broad spectrum of multiple, overlapping traits. Similarly, previous results are mostly limited to the Big Five personality traits, while personality can be organized hierarchically from general (e.g., Stability; DeYoung et al. 2002) to more specific traits (e.g., the Big Five). In this study, we investigate common and unique genetic influences on well-being and personality while taking their hierarchical organizations into account, by systematically testing competing theoretical independent and common pathway models. Data on the Big Five, life satisfaction, quality of life, self-rated health, loneliness and depression from 14,253 twins and their siblings from the Netherlands Twin Register are used. Multivariate extended twin models showed Neuroticism, Extraversion, and Conscientiousness to have the strongest genetic and environmental overlap with the well-being traits. The best-fitting theoretical model indicated that well-being and personality share substantial common etiological influences, yet that they also have their own personality-specific and wellbeing-specific influences, and trait-specific effects. Significant amounts of non-additive genetic influences on the traits' (co)variances was found. Little evidence was found for quantitative or qualitative sex differences. Taken together, our results suggest that well-being is more than a personality thing, which has important implications for studies trying to identify the genetic loci influencing personality and well-being.

References

DeYoung CG, Peterson JB, Higgins DM (2002) Higher-order factors of the Big Five predict conformity: Are there neuroses of health? Pers Individ Dif 33:533-552. https://doi.

org/10.1016/S0191-8869(01)00171-4

Weiss A, Bates TC, Luciano M (2008) Happiness is a personal(ity) thing: The genetics of personality and well-being in a representative sample. Psychol Sci 19:205-210. https://doi.org/

10.1 111/j.1467-9280.2008.02068.x

Grant Support: This work is supported by the European Research Council Consolidator Grant (ERC-2017-COG 771057 WELL-BEING PI Bartels).

\section{Pupil Size and Pupillary Light Reflex in Early Infancy: Heritability and Link to Neurodevelopmental and Psychiatric Genetic Liability in the general Population}

Ana Maria Portugal ${ }^{1}$, Mark J. Taylor ${ }^{2}$, Pär Nyström ${ }^{3}$, Charlotte Viktorsson ${ }^{4}$, Danyang $\mathrm{Li}^{1,5}$, Kristiina Tammimies ${ }^{1,5}$, Angelica Ronald ${ }^{6}$, Terje Falck-Ytter ${ }^{1,4,7}$

${ }^{1}$ Center of Neurodevelopmental Disorders (KIND), Centre for Psychiatry Research; Department of Women's and Children's Health, Karolinska Institutet \& Stockholm Health Care Services, Region Stockholm, Stockholm, Sweden

${ }^{2}$ Dept of Medical Epidemiology \& Biostatistics, Karolinska Institutet, Stockholm, Sweden

${ }^{3}$ Department of Psychology, Uppsala University, Uppsala, Sweden

${ }^{4}$ Development and Neurodiversity Lab (DIVE), Department of Psychology, Uppsala University, Uppsala, Sweden

${ }^{5}$ Astrid Lindgren Children's Hospital, Karolinska University Hospital, Region Stockholm, Sweden

${ }^{6}$ Department of Psychological Sciences, Birkbeck, University of London, London, UK

${ }^{7}$ Swedish Collegium for Advanced Study (SCAS), Uppsala, Sweden

Keywords Pupillometry, Twins, Polygenic risk score, Infancy, Development

The pupil regulates the amount of light reaching the retina and has basic visual sensory regulatory functions. Previous work links an atypical pupillary light reflex (pupil constriction to light) to neurodevelopmental and psychiatric conditions, which are highly heritable. However, there have been no twin studies of pupil responses in infancy or attempts to understand its genetic architecture. Here we estimated genetic and environmental influences on baseline pupil size and the pupillary light reflex in 510 infant twins assessed at 5 months (281 monozygotic and 229 dizygotic pairs), and the relationship with polygenic risk for neurodevelopmental and psychiatric disorders. Pupil size $\left(\mathrm{h}^{2}=.64 \mathrm{CI} .54\right.$ to .72$)$ and constriction response to light $\left(\mathrm{h}^{2}=.62, \mathrm{CI} .51\right.$ to .70$)$ were both highly heritable, and bivariate twin modelling indicated that the genetic factors showed modest overlap ( $\mathrm{rG}=.38$, CI .23 to .52 ). When analyzing genome-wide polygenic scores (GPSs), a statistically significant association between pupil size and the GPS for schizophrenia $\left(\beta=.15, \mathrm{p}=.024, \Delta \mathrm{R}^{2}\right.$ $=.016$ ) was found but there were no significant associations with the GPS for autism $(p>.25)$. Further, there were no associations between constriction response and GPSs. This study suggests that individual differences in pupil size and the pupil light reflex are heritable and provides initial evidence that pupil size may share some links with schizophrenia genetic liability. It underscores the possibilities of 
combining established behavioral genetic methods with experimental psychophysiological developmental assessments to further our understanding of human brain development.

Grant Support: The BabyTwins Study Sweden (BATSS) was supported by funding from Riksbankens Jubileumsfond and the European Union Initial Training Network BRAINVIEW.

\section{The Influence of Specialist Genes in Mathematical, Language and Reading Ability}

Francesca Procopio ${ }^{1}$, Margherita Malanchini ${ }^{1,2}$, Kaili Rimfeld ${ }^{1}$, Agnieszka Gidziela $^{1,2}$ \& Robert Plomin ${ }^{1}$

${ }^{1}$ Social, Genetic and Developmental Psychiatry Centre, Institute of Psychiatry, Psychology and Neuroscience, King's College London, London, UK

${ }^{2}$ Department of Psychology, Queen Mary University of London, London, UK

Keywords General cognitive ability, Intelligence, Mathematics, Reading, Polygenetic scores

Although a general factor of cognitive ability $(\mathrm{g})$ is well-established within the literature, both phenotypically and genetically, more than half of the variance of specific cognitive abilities is independent of $g$. We hypothesized that we could identify specific genetic effects on mathematical, language and reading ability independent of g. We used a sample of 5434 12-year-old twin pairs (1945 MZ, 1760 samesex DZ and 1729 opposite-sex DZ) from the Twins Early Development Study. The twins were assessed on verbal and nonverbal abilities (g) and mathematical, reading and language abilities using a battery of 14 tests. Using univariate twin analyses, we explored the genetic and environmental components of the three specific cognitive abilities phenotypically corrected for g. These results were complemented by bivariate Cholesky decomposition analyses between each of the specific cognitive abilities and $g$ to estimate specific genetic effects independent of g. Finally, we conducted comparable analyses using genome-wide polygenetic scores (GPS), beginning with the most recent IQ GPS (2018 'IQ3'). First, we correlated each GPS with each of the three g-corrected specific cognitive abilities. We then conducted 'GPS-by-subtraction' analyses that corrected IQ3 for $\mathrm{g}$ and correlated the corrected GPS with each specific cognitive ability.

\section{References}

Savage JE, Jansen PR, Stringer S, Watanabe K, Bryois J, de Leeuw CA et al. Genome-wide association meta-analysis in 269,867 individuals identifies new genetic and functional links to intelligence. Nature genetics 2018; 50(7): 912-919.

Grant Support: UK Medical Research Council (MR/M021475/1) with additional support from the US National Institutes of Health (AG04938)

\section{The Role of Prenatal Distress on Children's Early Social Competence: Disentangling Heritable and Environmental Influences}

Amanda M. Ramos ${ }^{1}$, Elizabeth A. Shewark ${ }^{2}$, David Reiss ${ }^{3}$, Leslie D. Leve ${ }^{4}$, Misaki N. Natsuaki ${ }^{5}$, Daniel S. Shaw ${ }^{6}$, Jody M. Ganiban ${ }^{7}$, and Jenae M. Neiderhiser ${ }^{8}$
${ }^{1}$ Department of Epidemiology, University of North Carolina-Chapel Hill, Chapel Hill, North Carolina, USA

${ }^{2}$ Department of Psychology, Michigan State University, East Lansing, Michigan, USA

${ }^{3}$ Child Study Center, Yale University, New Haven, Connecticut, USA

${ }^{4}$ Prevention Science Institute, University of Oregon, Eugene, Oregon, USA

${ }^{5}$ Department of Psychology, University of California, Riverside, Riverside, California, USA

${ }^{6}$ Department of Psychology, University of Pittsburgh, Pittsburgh, Pennsylvania, USA

${ }^{7}$ Department of Psychological and Brain Sciences, George Washington University, Washington, DC, USA

${ }^{8}$ Department of Psychology, The Pennsylvania State University, University Park, Pennsylvania, USA

Keywords Child social competence, Prenatal distress, Adoption design

Prenatal distress has been associated with lower levels of child social competence in early childhood in some studies. However, most studies cannot distinguish between genetic or environmental explanations for these findings. Using the Early Growth and Development Study $(N=561)$, a prospective parent-offspring adoption design, we examined inherited and prenatal influences on child social competence at 4.5 years as well as potential mediational pathways of toddler reactivity and dysregulation. Birth parent (BP) temperament (Emotion Dysregulation and Agreeableness) indexed inherited characteristics. Prenatal distress was assessed using birth mother reports of her anxiety and depressive symptoms during pregnancy. Adoptive parents reported on child anger proneness and ADHD symptoms at 18 and 27 months and social competence at 4.5 years old. We used structural equation modeling and found that $\mathrm{BP}$ emotion dysregulation was negatively associated with social competence at age 4.5 years $(\beta=-$ $.09, p<.05)$. Prenatal distress was not significantly related to social competence directly or indirectly via child reactivity or dysregulation. Child reactivity and dysregulation at 27 months were negatively associated with social competence $(\beta=-.15, p<.05 ; \beta=-.20, p<$ .01 , respectively). BP emotion dysregulation was positively associated with prenatal distress $(\beta=.18, p<.01)$.

These findings support the importance of early regulatory behaviors and heritable factors for child social competence, but not prenatal distress. The findings reflect the importance of examining prenatal effects within a genetically informed design.

Grant Support: R01 HD042608 NICHD; R01 DA020585, R01 DA045108 NIDA; R01 MH092118 NIMH; UG3/UH3 OD023389 from the Office of the Director, NIH; R305B090007 IES; F32ES031832 NIEHS; F31HD089582 NICHD

\section{The Mediating Role of Reading-Related Endophenotypes in the Association Between Genes and Reading Skills}

Alexandra Remon ${ }^{1}$, Sophie Aubé ${ }^{1}$, Bei Fang ${ }^{1}$, Frank Vitaro ${ }^{2}$, Mara Brendgen $^{3}$, Isabelle Ouellet-Morin ${ }^{2}$, Michel Boivin ${ }^{1,4}$, Sara Mascheretti ${ }^{1,5}$, Ginette Dionne ${ }^{1}$

${ }^{1}$ Laval University, École de Psychologie, Québec, QC, Canada

${ }^{2}$ University of Montréal, Montréal, QC, Canada

${ }^{3}$ University of Québec, Montréal, QC, Canada

${ }^{4}$ Tomsk State University, Tomsk, Russia

${ }^{5}$ Scientific Institute, IRCCS E. Medea, Child Psychopathology Unit, Bosisio Parini, Lecco, Italy 
Keywords Candidate genes, Reading skills, Endophenotypes, Mediation, Academic achievement

Reading acquisition relies upon several reading-related neurocognitive skills (Bailey \& al., 2018). Although reading skills have already been associated with candidate genes and reading-related endophenotypes (EPs), processes explaining the relationship between candidate genes and reading remain poorly studied (Mascheretti \& al., 2018). It has been suggested that EPs may mediate the pathway between genes and reading skills (Mascheretti et al., 2020). Here, we replicate previous findings by testing four EPs (i.e., phonological awareness, rapid automatized naming (RAN), rapid bimodal temporal processing, rapid auditory processing) and 14 markers spanning five reading-related candidate genes (i.e., DYX1C1, DCDC2, KIAA0319, ROBO1 and GRIN2B) using a multiple-predictor/multiple-mediator framework in a sample of 253 twins ( $80 \mathrm{MZ}$ and $173 \mathrm{DZ}$ ). Results showed that RAN mediates the pathway from GRIN2B-rs 2192973 to reading. Carrying the minor ' $A$ ' allele correlates with longer reaction times in RAN, which, in turn, correlates with lower reading comprehension skills. Our results support the use of EPs to explain the complex pathways between genes and behavior.

\section{References}

Bailey, S. K., Aboud, K. S., Nguyen, T. Q. \& Cutting, L. E. (2018). Applying a network framework to the neurobiology of reading and dyslexia. Journal of neurodevelopmental disorders, 10(1), 1-9. https://doi.org/10.1186/s11689-018-9251-z.

Mascheretti, S., Gori, S., Trezzi, V., Ruffino, M., Facoetti, A. \& Marino, C. (2018). Visual motion and rapid auditory processing are solid endophenotypes of developmental dyslexia. Genes Brain Behav, 17(1), 70-81. https://doi.org/10.1111/gbb.12409.

Mascheretti, S., Riva, V., Feng, B., Trezzi, V., Andreola, C., Giorda, R., ... \& Facoetti, A. (2020). The Mediation Role of Dynamic Multisensory Processing Using Molecular Genetic Data in Dyslexia. Brain sciences, 10(12), 993. https://doi.org/10.3390/brain sci10120993

Grant Support: This research is supported by the Canadian Institutes of the Social and Humanities Research Council and the Quebec Research Fund- Society and Culture.

\section{Assortative Mating for Autistic Traits, Systemizing, and Theory of Mind}

Gareth Richards ${ }^{1,2}$, Simon Baron-Cohen ${ }^{2}$, Varun Warrier ${ }^{2}$, Shanhong Luo ${ }^{3}$, Robin Dunbar ${ }^{4}$, Emily Jackson ${ }^{1}$, Hannah Proctor ${ }^{1}$, Holly Stokes ${ }^{5}$ Eva Lee ${ }^{1}$, Ben Mellor ${ }^{1}$, Jessica Davies ${ }^{5}$,

Laura Gee $^{5}$, \& John Galvin ${ }^{5}$

${ }^{1}$ School of Psychology, Newcastle University, Newcastle upon Tyne, Tyne and Wear, UK

${ }^{2}$ Autism Research Centre, University of Cambridge, Cambridge, Cambridgeshire, UK

${ }^{3}$ Department of Psychology, University of North Carolina Wilmington, Wilmington, North Carolina, USA

${ }^{4}$ Department of Experimental Psychology, University of Oxford, Oxford, Oxfordshire, UK

${ }^{5}$ School of Psychology, Birmingham City University, Birmingham, West Midlands UK

Keywords Assortative mating, Autism, Autistic traits, Systemizing, Theory of Mind
We aimed to test the hypothesis that traits associated with autism spectrum conditions are subject to assortative mating. Study 1 examined self-reported autistic traits (Autism Spectrum Quotient [AQ]), systemizing (Systemizing Quotient-Revised [SQ-R]), and empathizing (Empathy Quotient [EQ]), as well as behavioral measures related to socio-perceptual Theory of Mind (Reading the Mind in the Eyes Test [RMET]) and systemizing (Embedded Figures Task $[E F T])$. Variable-centered analyses revealed couple-similarity correlations for AQ $(r[102]=0.305, p=0.002)$, SQ-R $(r[101]=0.263, p=$ $0.007)$, RMET $(r[53]=0.438, p<0.001)$ and EFT $(r[56]=0.423, p<$ $0.001)$, but not EQ $(r[100]=-0.018, p=0.860)$. Further analysis suggested people pair with others more similar than chance (initial assortment) rather than become alike during a relationship (convergence), and that they seek out similar partners (active assortment) rather than pair with similar people due to social stratification (social homogamy). We next used couple-centered analyses to compare similarity scores between actual couples and the average of all other possible male-female pairings within the dataset. Actual couples were more similar for AQ $(d=0.250 p=0.002)$, SQ-R $(d=0.211, p=$ $0.007)$, RMET $(d=0.393, p=0.007)$ and EFT $(d=0.365, p=0.006)$, but not EQ $(d=-0.002, p=0.980)$. In Study 2, we replicated the variable-centered $(r[94]=0.284, p=0.005)$ and couple-centered results $(d=0.253, p=0.032)$ for socio-perceptual Theory of Mind (RMET). However, there was no evidence for assortment for sociocognitive Theory of Mind when using the Stiller-Dunbar Stories Task (variable-centered: $r[98]=0.048, p=0.635$; couple-centered, $d=$ $0.012, p=0.917)$. Random-effects meta-analysis $(\mathrm{k}=16, \mathrm{n}=5,892)$ confirmed a significant couple-similarity correlation for autistic traits, $r=0.186, p<0.0001$. These findings support the assortative mating theory of autism and should be considered when estimating heritability.

Grant Support: SBC and VW received funding from the Wellcome Trust, Innovative Medicines Initiative 2 Joint Undertaking (JU), the Autism Research Trust, SFARI, the Templeton World Charitable Foundation, and the National Institute for Health Research (NIHR) Collaboration for Leadership in Applied Health Research and Care East of England at Cambridgeshire and Peterborough NHS Foundation Trust. JG was supported by a Birmingham City University Small Development Grant (ML/ZM/GP; Ref: SDG 17-18-1.2).

\section{A Protective Role of Internalizing Symptoms in Adolescent Substance Use: Popularity and Harm Avoidance as Potential Mediators}

Maya M. Rieselbach ${ }^{1,2}$, Shelley Gresko ${ }^{1,2}$, Robin P. Corley ${ }^{2}$, John K. Hewitt ${ }^{1,2}$, and Soo Hyun Rhee ${ }^{1,2}$

${ }^{1}$ Department of Psychology and Neuroscience, University of Colorado Boulder, USA

${ }^{2}$ Institute for Behavioral Genetics, University of Colorado Boulder, USA

Keywords Substance use, Internalizing, Popularity, Harm avoidance, Adolescent

Psychological symptoms are generally considered to pose risk for substance use, yet findings suggest that internalizing symptoms may protect from adolescent substance use after controlling for co-occurring externalizing symptoms. The present study examined two potential mediators of the internalizing-specific association with substance use: popularity and harm avoidance. Adolescent substance use typically occurs in social contexts, so more socially-connected teens may have more opportunities to access substances. Internalizing 
symptoms may be associated with reduced popularity with peers, which may in turn lead to fewer connections through which to obtain substances. Also, internalizing symptoms may be positively associated with harm avoidance (i.e., depressed or anxious adolescents may be actively avoiding potential risks associated with substance use). The present study used data from the Colorado Longitudinal Twin Study and Colorado Adoption Project. Assessments of internalizing, externalizing, social competence, and harm avoidance were collected between the ages of 7 and 17. Substance use was assessed at age 17 and analyses included a latent substance use factor with loadings on alcohol, nicotine, cannabis, and other drug use. Results supported an internalizing-specific negative association with substance use. Popularity was negatively correlated with both substance use and internalizing, suggesting that it does not mediate the association between internalizing and substance use. Harm avoidance was positively correlated with internalizing and negatively correlated with substance use, yet mediation analyses did not support harm avoidance as a mediator of the association between internalizing and substance use. Future research is needed to explore the protective influence of internalizing on substance use.

Grant Support: National Institutes of Health Grants AG046938, DA011015, DA038504, and DA017637

\section{The Consequences of the COVID-19 Crisis for the Mental Health of Young Adult Twins in England and Wales}

Kaili Rimfeld ${ }^{1}$, Margherita Malanchini ${ }^{1,2}$, Ryan Arathimos ${ }^{1}$, Agnieszka Bubel $^{1,2}$, Oliver Pain ${ }^{1}$, Andrew McMillan ${ }^{1}$, Rachel Ogden ${ }^{1}$,Louise Webster ${ }^{1}$, Nicholas G. Shakeshaft ${ }^{1}$, Kerry L. Schofield ${ }^{1}$, Jean-Baptiste Pingault ${ }^{4}$, Andrea G. Allegrini ${ }^{4}$, Argyris Stringaris ${ }^{4}$, Sophie von Stumm ${ }^{5}$,

Cathryn Lewis ${ }^{1} \&$ Robert Plomin ${ }^{1}$

${ }^{1}$ Social, Genetic and Developmental Psychiatry Centre, Institute of Psychiatry, Psychology and Neuroscience, King's College London, London, UK

${ }^{2}$ Department of Psychology, Queen Mary University of London, London, UK

${ }^{3}$ Clinical, Educational \& Health Psychology, Division of Psychology $\&$ Language Sciences, Faculty of Brain Sciences, University College London

${ }^{4}$ Mood, Brain \& Development Unit, Emotion and Development Branch, National Institute of Mental Health

${ }^{5}$ Psychology in Education Research Centre, Department of Education, University of York

\section{Keywords COVID-19, Pandemic, Lockdown, Mental health}

The COVID-19 pandemic has impacted the lives of many, not only through the infection itself, but also through full or partial isolation (lockdown). Here we investigated how the COVID-19 pandemic and unprecedented global lockdown affected the mental health of young adults in England and Wales. We compared the mental health symptoms of 4000 twins in their mid-twenties prior to COVID-19 crises (2018) to those of a four-wave longitudinal data collection during the pandemic in April, July, and October 2020, and in March 2021. The average changes in mental health symptoms were small and mainly occurred over the 2-year period from 2018 to March 2020 (average Cohen $\mathrm{d}=0.24$ ) but we did not observe trends in worsening mental health during the pandemic. Twin analyses indicated that aetiology of individual differences did not change during the lockdown. The average heritability was $33 \%$ across 5 waves of assessment, and the average genetic correlation between $\mathrm{T} 1$ and $\mathrm{T} 2-$ T5 was .95 , indicating that the genetic effects of $\mathrm{T} 1$ are substantially correlated with genetic effects at T2-T5. However, we show that the COVID-19 pandemic disproportionately affected the mental health of young adults who at $\mathrm{T} 1$ had pre-existing vulnerabilities. We conclude that on average young adults in the UK are remarkably resilient to the effects of the pandemic and associated lockdown. However, young people with pre-existing mental health problems are adversely affected. Preventive interventions for young adults should be targeted to those with existing vulnerabilities during and after a crisis, and this is especially important is young adulthood because it is a tipping point for life-long psychological problems.

Grant Support: UK Medical Research Council (MR/M021475/1) with additional support from the US National Institutes of Health (AG04938); Sir Henry Wellcome Postdoctoral Fellowship

\section{Discordant-Sibling Designs and Applications for Differential Psychology}

\author{
Hannah Robertson ${ }^{1}$, Joseph Lee Rodgers ${ }^{2}$, S. Mason Garrison ${ }^{1}$ \\ ${ }^{1}$ Department of Psychology, Wake Forest University, Winston Salem, \\ NC USA \\ ${ }^{2}$ Department of Psychology and Human Development, Vanderbilt \\ University, Nashville, TN, USA
}

Keywords Causal Inference, Differential Psychology, GeneticallyInformed Designs; Quasi-Experimental Designs; Research Methods

Randomized experiments are the "gold standard" for inferring causation and are designed to collect covariate information (Rubin, 2008). Yet, many questions cannot be answered with experiments practically or ethically. Often, potential confounds are controlled statistically as covariates in quasi-experimental designs. The typical use of covariates does not control for many systematically confounded gene-and-environmental effects. Poverty, health, and personality all covary with gene-and-environmental effects, so much so that using covariates can create bias. We advocate for using genetically-informed designs as they support better causal inference without random assignment and adjust for most gene-and-environmental elements. We illustrate the discordant kinship model (Garrison \& Rodgers, 2016). Differences between kin pairs explicitly distinguish within-family variance from between-family, and control for all background variance linked to gene-and-environmental differences. We present three illustrations with personality predictors, specifically conscientiousness and neuroticism interacting with socio-economic status from the NLSY79 dataset. All three illustrations found significant associations when using covariate-based approaches. After addressing systematic confounding within the discordant-kinship approach, the results diverged. Understanding the relationship between poverty, mental health, and personality as related to genetic or environmental effects can aid in the overall health of a person.

\section{References}

Garrison, S. M., \& Rodgers, J. L. (2016). Casting doubt on the causal link between intelligence and age at first intercourse: A crossgenerational sibling comparison design using the NLSY. Intelligence

Donald B. Rubin (2008) Comment: The Design and Analysis of Gold Standard Randomized Experiments, Journal of the American Statistical Association 


\section{Pubertal Timing, Child Body Mass Index and the Obesogenic Environment}

Olivia C. Robertson ${ }^{1}$, Kristine Marceau ${ }^{1}$, Misaki N. Natsuaki ${ }^{2}$, Daniel S. Shaw ${ }^{3}$, David Reiss ${ }^{4}$, Leslie D. Leve ${ }^{5}$,

Jenae M. Neiderhiser ${ }^{6}$, Jody M. Ganiban ${ }^{7}$

${ }^{1}$ Department of Human Development and Family Studies, Purdue University

${ }^{2}$ Department of Psychology, University of California, Riverside

${ }^{3}$ Department of Psychology, University of Pittsburgh

${ }^{4}$ Child Study Center, Yale University

${ }^{5}$ Prevention Science Institute, University of Oregon

${ }^{6}$ Department of Psychology, Penn State University

${ }^{7}$ Department of Psychology, George Washington University

Keywords Pubertal timing, Gene by Environment Interaction, Childhood Obesity, Obesogenic environment

The obesogenic family environment is considered a key contextual factor for child body mass index (BMI), and elevated child BMI is a strong predictor for early pubertal timing. As shared genetic influences and metabolic processes underlie the development of obesity and pubertal timing, and may be influenced by obesogenic environmental factors, we expect that family obesogenic environments may exacerbate heritable risk on pubertal timing. Using cohort I $(n=361)$ of the Early Growth and Development Study, a US-based sample of children adopted into non-relative families at birth, we examined whether the obesogenic family environment moderated the effect of heritable influences (birth parent BMI) for earlier puberty in 11-yearold boys and girls. Obesogenic family environment was quantified by latent profiles derived from indicators of adoptive parent characteristics, feeding behavior, and parenting: average $(n=276)$, weight concerned $(n=52)$, and low control $(n=31)$. In girls, genetic influences were only associated with early pubertal timing in the weight concerned profile. In boys, genetic influences predicted earlier puberty in the average and weight concerned, but not the low control profile. No mean differences in pubertal timing were found for boys across profiles. In girls, the low control had later pubertal timing than the weight concerned profile. However, in omnibus tests, profiles could be constrained for mean pubertal timing and the effect of heritable influences on pubertal timing for both boys and girls without a decrement in model fit. Overall, results suggest weak evidence of genetic by family obesogenic environment interaction for puberty.

Grant Support: The funding for this study was provided by grant R01 HD042608 from the Eunice Kennedy Shriver National Institute of Child Health \& Human Development and the National Institute on Drug Abuse, NIH, U.S. PHS (PI Years 1-5: David Reiss, MD; PI Years 6-10: Leslie Leve, Ph.D.), R01 DA020585 from the National Institute on Drug Abuse, the National Institute of Mental Health and OBSSR, NIH, U.S. PHS (PI: Jenae Neiderhiser, Ph.D.), R01 MH092118 from the National Institute of Mental Health, NIH, U.S. PHS (PIs: Jenae Neiderhiser, Ph.D. and Leslie Leve, Ph.D.), R01 DK090264 from the National Institute of Diabetes and Digestive and Kidney Disease, NIH, U.S. PHS (PI: Jody Ganiban, Ph.D.), R56 HD042608 from the Eunice Kennedy Shriver National Institute of Child Health \& Human Development, NIH, U.S. PHS (PI: Leslie Leve, Ph.D.), R01 DA045108 (PI: Jenae Neiderhiser, Ph.D.), UH3OD023389 (PIs: Leslie Leve, Ph.D., Jenae Neiderhiser Ph.D., Jody Ganiban Ph.D.) and K01 DA039288 (PI: Kristine Marceau, Ph.D.).

\section{Does Maternal Smoking During Pregnancy Moderate the Effect of Genes on Offspring's Smoking Behavior?}

Pamela N. Romero Villela ${ }^{1,2^{*}}$, Jared C. Balbona ${ }^{1,2}$, Richard Border, Ph.D. ${ }^{3}$, Matthew C. Keller, Ph.D. ${ }^{1,2}$, Luke M. Evans, Ph.D. ${ }^{1,5}$, Marissa A. Ehringer, Ph.D. ${ }^{1,4}$

${ }^{1}$ Institute for Behavioral Genetics, University of Colorado, Boulder, Colorado, USA ${ }^{2}$

${ }^{2}$ Department of Psychology, University of Colorado,

Boulder, Colorado

${ }^{3}$ Departments of Neurology and Computer Science, University of California, Los Angeles

${ }^{4}$ Department of Integrative Physiology, University of Colorado, Boulder, Colorado

${ }^{5}$ Department of Ecology \& Evolutionary Biology, University of Colorado, Boulder, Colorado

*Corresponding Author: Pamela Romero Villela, Institute for Behavioral Genetics, Boulder, CO 80309; pamela.romerovillela@colorado.edu

Keywords Genetics, Epigenetics, Smoking, GxE, Maternal smoking during pregnancy

Maternal smoking during pregnancy (MSP) is a strong predictor of later offspring nicotine use. However, not all children exposed to nicotine prenatally later go on to smoke, suggesting that the effect of MSP may be moderated by genetic factors (Buka, Shenassa, \& Niaura, 2003). Due to limited sample sizes and statistical power, few studies have investigated this potential interaction between genetics and maternal smoking during pregnancy. We attempt to address this gap using the UK Biobank to examine potential genetic interactions with maternal smoking to predict a wide range of smoking phenotypes: cigarettes per day, smoking initiation, smoking cessation, and birth weight. We used plink2 (Purcell et al., 2007) to conduct a genome-wide interaction study to assess if genome-wide loci moderated the effect of MSP on unrelated offspring of European descent $(n=116,442)$. Our results failed to find loci that reached genome-wide significance for the interaction with maternal smoking. It is possible that even with a large sample size such as UK Biobank, the power to detect such interactions at the genome-wide level is limited. Future analyses will investigate gene-by-environment and polygenic risk score-by-environment interactions.

\section{References}

Buka, S. L., Shenassa, S. D. E. D., \& Niaura, S. D. R. (2003). Article Elevated Risk of Tobacco Dependence Among Offspring of Mothers Who Smoked During Pregnancy: A 30-Year Prospective Study. Am J Psychiatry (Vol. 160). Retrieved from http://ajp.psychiatryonline.org Purcell, S., Neale, B., Todd-Brown, K., Thomas, L., Ferreira, M. A. R., Bender, D., ... Sham, P. C. (2007). PLINK: a tool set for whole-genome association and population-based linkage analyses. American Journal of Human Genetics, 81(3), 559-575. https://doi.org/10.1086/519795

Grant Support: Not applicable. 


\section{Investigating Gene-Environment Correlation with a Biometric Cross-Lagged Panel Model: The Case of Parental Educational Expectations and Child's Learning Motivation}

\author{
Mirko Ruks ${ }^{1}$ \\ ${ }^{1}$ TwinLife Project and Faculty of Sociology, University of Bielefeld, \\ Bielefeld, Germany
}

Keywords Gene-environment correlation, Educational expectation, Learning motivation, Longitudinal twin model, Longitudinal twin model

One well-established finding of sociological research is the social inequality of educational opportunity (IEO). However, the question of the underlying mechanisms is empirically still not sufficiently clear. Two often discussed candidates are the "compensatory advantage" and "multiplicative advantage" mechanisms, according to which socio-economically advantaged families tend to compensate for children's early disadvantages or reinforce early endowments. However, at least two problematic aspects in current research on compensatory and multiplicative advantages can be identified: (1) Most studies only examine whether the relevance of children's characteristics for their educational success varies with social background, while concrete practices through which compensation and multiplication might operate are not identified. (2) Research generally remains at a purely phenotypic level, ignoring behavioral genetics research on gene-environmental interplay in general and gene-environment correlation (rGE) in particular. While the theoretical concept of rGE is well-established in the behavioral genetics literature, the discussion about empirical approaches to model rGE processes using longitudinal twin models has started only recently. In this paper, I try to enhance the sociological research on IEO drawing on this theoretical and empirical literature on rGE to address both aspects. Using data from the German twin study "TwinLife", a biometric crosslagged panel model is computed to test (1) whether parents compensate or reinforce for their children's early learning motivation via their own educational expectations and if these patterns vary with parental socio-economic status, and (2) to what extent parental educational expectations are evoked by the children's genetic disposition for learning motivation, indicating rGE.

Grant Support: This work is supported by funding from the German Research Foundation (DFG) as long-term project TwinLife (DI 759/11-3).

\section{Genetic and Environmental Influences on Tinnitus over 12 Years in Vietnam-Era Veteran Twins}

\author{
Mitali Sakharkar ${ }^{1}$, Kristy Cuthbert ${ }^{1}$, Ruth E. McKenzie ${ }^{1,3}$, \\ Rosemary Toomey ${ }^{1}$, Matthew S. Panizzon ${ }^{2}$, Carol E. Franz ${ }^{2}$, \\ William S. Kremen ${ }^{2}$, Michael J. Lyons ${ }^{1}$ \\ ${ }^{1}$ Department of Psychological \& Brain Science, Boston University, \\ Boston, MA, USA. \\ ${ }^{2}$ Department of Psychiatry and Center for Behavioral Genetics of \\ Aging, University of California, San Diego, La Jolla, CA, USA. \\ ${ }^{3}$ Applied Human Development and Community Studies, Merrimack \\ College, North Andover, MA, USA.
}

Keywords Heritability, Genetics, Tinnitus, Twin study

Tinnitus has a higher prevalence in veteran and aging populations ${ }^{1}$. There are few studies examining longitudinal genetic models of tinnitus $^{2}$, and no known studies have utilized a sample of aging male veterans. This poster will examine longitudinal ACE models for tinnitus in the Vietnam Era Twin Study of Aging. $468 \mathrm{MZ}$ and $317 \mathrm{DZ}$ male twin pairs self-reported tinnitus (yes or no) at mean ages of 56, 62, and 68 years. Longitudinal ACE twin models of tinnitus were generated to examine genetic and environmental influences over three time points (T1, T2, T3). Tetrachoric correlations for T1-T3 tinnitus were 0.78 from $\mathrm{T} 1-\mathrm{T} 2,0.80$ from $\mathrm{T} 2-\mathrm{T} 3$, and 0.67 from $\mathrm{T} 1-\mathrm{T} 3$ (all $\mathrm{p}<.001$ ). An AE model best fit the data; there was no evidence for the influence of shared environment on tinnitus. Additive genetic factors significantly accounted for $28.5 \%, 46.6 \%$, and $44.3 \%$ of the variance at $\mathrm{T} 1, \mathrm{~T} 2, \mathrm{~T} 3$, respectively, with remaining variance attributed to non-shared environment. Full results from a trivariate model (including genetic correlations across times) will be included in the poster. Results indicate significant non-shared environmental and genetic influences on tinnitus over time. Most of the variance in tinnitus at age 56 is determined by unique environmental influences. However, there is a suggestion in these preliminary findings that genetic influences may become equally influential as subjects transition from middle to early old age.

\section{References}

Folmer, R. L., McMillan, G. P., Austin, D. F., \& Henry, J. A. (2011). Audiometric thresholds and prevalence of tinnitus among male veterans in the United States: data from the National Health and Nutrition Examination Survey, 1999-2006. Journal of Rehabilitation Research \& Development, 48(5)

Vona, B. (2019). Heritability and tinnitus. JAMA OtolaryngologyHead \& Neck Surgery, 145(3), 229-230.

\section{A Genetically-Informed Test of the Biopsychosocial Theory of Borderline Personality Disorder: Informant and Sex Effects}

Sang, E. ${ }^{1}$, McGue, M. ${ }^{2}$, Iacono, W.G. ${ }^{2}$, Bornovalova, M.A. ${ }^{1}$

${ }^{1}$ Department of Psychology, University of South Florida, Tampa, Florida, USA.

${ }^{2}$ Department of Psychology, University of Minnesota, Minneapolis, Minnesota, USA

Keywords Borderline Personality Disorder, Parenting, Direction of Causation, Informant Effects

Background: Parent-child transactions are at the heart of the biopsychosocial theory of borderline personality disorder (BPD). However, few studies have examined the longitudinal, bidirectional relationship between BPD and parenting factors, and fewer still have attempted to differentiate reciprocal causation from common genetic liabilities.

Methods: We used a longitudinal, genetically informed design consisting of mixed-sex monozygotic, dizygotic, and adoptive sibling pairs ( $\mathrm{n}=4996,52.7 \%$ female) followed longitudinally from age 14 to 17 . We examined the developmental patterns of BPD traits (Minnesota Borderline Personality Scale) and parenting factors (parentchild conflict and involvement, Parental Environment Questionnaire), as well as gene-environment correlations and causal effects across child sex and informant.

Results: Longitudinal direction of causation models indicated bidirectional influences between all three ratings of parenting (child, mother, and father) and BPD traits across child sex. At both age 14 and 17, BPD traits and parenting factors showed high genetic correlations across sex and informants. 
Conclusions: The heritability of parenting is in large part accounted for by genetic factors on BPD. However, there is evidence of reciprocal causation between BPD traits and parenting factors as well. Together, these results provide some support for the biopsychosocial theory of BPD while highlighting the role of personenvironment correlations.

Grant Support: This work was supported by National Institute on Drug Abuse Grant DA 05147, National Institute on Alcohol Abuse and Alcoholism Grants AA09367 and AA015621, and the National Institute on Mental Health MH066140-10.

\section{Gametic Phasing in a Sample of Distantly Related Individuals Using Segments Shared Identical by Descent (IBD).}

\author{
Emmanuel Sapin ${ }^{1}$ and Matthew C Keller ${ }^{1,2}$ \\ ${ }^{1}$ Institute for Behavioral Genetics, University of Colorado at Boulder, \\ Boulder, Colorado, USA \\ ${ }^{2}$ Department of Psychology, University of Colorado at Boulder, \\ Boulder, Colorado, USA
}

\section{Keywords Gametic Phasing, IBD Segment, Clustering}

We define "gametic phasing" as the discrimination of the paternal from maternal haplotypes in an individual. Gametic phasing with genome-wide data is straight-forward when one or both parents are included in the sample, but it has not been solved in a sample of unrelated individuals in which genealogical data is unavailable. In the current study, we show how gametic phasing can be accomplished in a sample of conventionally unrelated (i.e., distantly related) individuals with unknown genealogies using segments shared identical by descent (IBD). We show that clustering of IBD sharing among corelatives of a focal individual into two clusters can allow gametic phasing of the focal individual. By further incorporating SNPs on the mitochondria and Y-chromosome, the paternal and maternal autosomal haplotypes can be distinguished. Such gametic phasing can serve a variety of functions, including estimates of how long assortative mating has occurred in a population, estimates of genetic nurture and vertical transmission without parental data, and parent-of-origin effects.

Grant Support: MH100141 (Keller)

\section{Trans-Ancestry GWAS Meta-analysis of Tobacco and Alcohol Use}

Gretchen Saunders ${ }^{1}$, GWAS \& Sequencing Consortium of Alcohol and Nicotine Use (GSCAN)

${ }^{1}$ Psychology, University of Minnesota, Minneapolis, MN

Keywords Alcohol, Nicotine, GWAS, Trans-ancestry

The use and abuse of nicotine and alcohol account for $>100$ million disability-adjusted life years across the globe, constituting one of the world's leading public health problems. Despite this, the vast majority of genome-wide association studies thus far have been restricted to individuals of European ancestry, representing $<1 \%$ of known worldwide genetic variation. Here, we leveraged a trans-ancestry GWAS of nicotine and alcohol use from up to 3.4 million individuals from 81 studies with recent ancestry from Africa $(\mathrm{N}=121,858)$,
America (285,155), East Asia (298,624), and Europe $(2,669,029)$. Overall, we identified 1449 loci and 3842 conditionally independent variants associated with our five substance use phenotypes: 33 loci (39 variants) for age of initiation of regular smoking, 738 (2489) for smoking initiation, 138 (245) for cigarettes per day, 132 (217) for smoking cessation, and 408 (852) for alcoholic drinks per week. Using trans-ancestry fine-mapping methods, we identified 653 loci with $90 \%$ credible intervals containing $<5$ variants. Approximately $18 \%$ of sentinel variants showed differential effects across ancestry. Polygenic risk score prediction accuracy in European ancestries ranged from 1.2 to $7.7 \%$, which attenuated to $0.6-5.1 \%$ in the remaining ancestries. Taken together, our results highlight the value of diverse ancestry inclusion in genetic studies.

Grant Support: NIDA T32DA050560; NIDA R01DA037904; NIDA R01DA044283

\section{Polygenic Risk for Psychiatric Disorder Reveals Distinct Association Patterns Across Social Behaviour in the General Population}

Fenja Schlag $^{1}$, Andrea Allegrini ${ }^{2,3}$, Jan Buitelaar ${ }^{4,5,6}$, Ellen Verhoef ${ }^{1}$, Marjolein van Donkelaar ${ }^{1}$, Robert Plomin ${ }^{2}$, Kaili Rimfeld ${ }^{2}$, Simon E. Fisher ${ }^{1,4}$, Beate St Pourcain ${ }^{1,4,7}$

${ }^{1}$ Language and Genetics, MPI for Psycholinguistics, Wundtlaan 1, 6525 XD Nijmegen, The Netherlands

${ }^{2}$ Social, Genetic and Developmental Psychiatry Centre, Institute of Psychiatry, Psychology \& Neuroscience, King's College London, Memory Ln, Camberwell, London SE5 8AF, London, UK

${ }^{3}$ Psychology and Language Sciences, University College London, 26 Bedford Way, Bloomsbury, London WC1H 0AP, London, UK

${ }^{4}$ Donders Institute for Brain, Cognition and Behaviour, Radboud University, Kapittelweg 296525 EN Nijmegen, The Netherlands

${ }^{5}$ Karakter Child and Adolescent Psychiatry University Centre, Reinier Postlaan 12, 6525 GC Nijmegen, The Netherlands

${ }^{6}$ Department of Cognitive Neuroscience, Radboud University Medical Center, Geert Grooteplein 21, 6525 EZ Nijmegen, The Netherlands

${ }^{7} \mathrm{MRC}$ Integrative Epidemiology Unit, University of Bristol, Oakfield House, Oakfield Grove, Bristol, BS8 2BN, UK

${ }^{8}$ Population Health Sciences, University of Bristol, 5 Tyndall Avenue, Bristol, BS8 1UD, UK

Keywords Psychiatric disorder, Polygenic risk scoring, Social behaviour, Genetic heterogeneity

Many complex neurodevelopmental conditions show a spectrum of social-behavioural difficulties. These are thought to lie on a heritable behavioural continuum that is shared with social behaviour in the general population. However, genetic continuity with psychiatric illness may vary for different social-behavioural symptoms. In this study, we systematically investigated differences in polygenic association patterns for attention-deficit/hyperactivity disorder (ADHD), autism spectrum disorders (ASD), bipolar disorder (BP), major depression (MD) and schizophrenia, across a spectrum of different social-behavioural symptoms. Polygenic risk scores for ADHD, ASD, $\mathrm{BP}, \mathrm{MD}$, and schizophrenia, as informed by publicly available genome-wide summary statistics from large consortia, were each regressed on longitudinally assessed low-prosociality and peer problem scores in two UK general population-based/community-based cohorts (ALSPAC, $\mathrm{N} \leq 6174,4-17$ years; TEDS, $\mathrm{N} \leq 7112$, 4-16 years; parent- and teacher-reports; negative binomial regressionmodels). Across ALSPAC and TEDS, we replicated univariate 
polygenetic associations between social behaviour and risk of ADHD, MD, and schizophrenia, but not ASD and BP. Modelling combined univariate genetic effects with meta-regression revealed variation in polygenic links across age, reporters and, especially, social trait. We identified a prevailing association of peer problems with ADHD, MD, and, once meta-analytically increasing power, also for ASD, while schizophrenia was solely associated with low-prosociality. The genetic association patterns for each disorder clustered distinctly across age-, reporter- and, especially, trait-specific symptoms of social behaviour investigated across 13 years of child and adolescent development. Our findings suggest marked differences in the genetic aetiology of psychiatric disorders across the spectrum of social difficulties.

\section{Deliberately Divided: The Controversial 1960s-70s Study of Twins and Triplets Adopted Apart}

\author{
Nancy L. Segal ${ }^{1}$ \\ ${ }^{1}$ Department of Psychology, California State University, Fullerton, \\ California, USA
}

\section{Keywords Twins, Triplets, Controversy ethics, Legality}

An in-depth look at the controversial twin study conducted in New York City, in the 1960s-70s, is presented. Material for this talk is drawn from my forthcoming book, Deliberately Divided: Inside the Controversial Study of Twins and Triplets Adopted Apart (Segal, October 2021). The riveting story of the study's origins, investigators, sealed files and questionable ethics are described. Detailed life histories of the participating pairs are also be provided. It is not surprising that this study was not well-known among scholars or the general public until it became the focus of two recent documentary films, The Twinning Reaction (Shinseki, 2017) and Three Identical Strangers (Wardle, 2018); even now, many twin and adoption researchers are unaware of this work. That is because the few published case studies were largely limited to psychoanalytic journals and meetings, and described briefly as part of a book. Responses to the study and to the films from academic and non-academic audiences have varied from outrage and sadness to enthusiasm and support. Ethical and legal inquiries, conferences and discussion groups have followed, and others will take place later this year. However, these events cannot capture or convey the full history, complexities and consequences of this study that a book can accomplish.

\section{References}

Segal, N.L. Deliberately divided: Inside the controversial study of twins and triplets adopted apart. Lanham, MD: Rowman \& Littlefield (in press, October 2021).

Shinseki, L. (2017). The Twinning Reaction. U.S.: Fire Horse Pictures.

Wardle, T. (2018). Three Identical Strangers. U.K.: Raw.

\section{Patterns of Brain Asymmetry Associated with Polygenic Risks for Autism and Schizophrenia Implicate Language and Executive Functions but not Brain Masculinization}

Zhiqiang Sha ${ }^{1}$, Dick Schijven ${ }^{1}$, Clyde Francks ${ }^{1,2}$

${ }^{1}$ Language and Genetics Department, Max Planck Institute for Psycholinguistics, Nijmegen, The Netherlands

${ }^{2}$ Donders Institute for Brain, Cognition and Behaviour, Radboud University, Nijmegen, The Netherlands

Keywords Brain asymmetry, Genetics, Structural MRI, Autism, Schizophrenia

Autism spectrum disorder (ASD) and schizophrenia have been conceived as partly opposing disorders in terms of systemizing versus empathizing cognitive styles, with resemblances to male versus female average sex differences. Left-right asymmetry of the brain is an important aspect of its organization that shows average differences between the sexes, and can be altered in both ASD and schizophrenia. Here we mapped multivariate associations of polygenic risk scores (PRS) for ASD and schizophrenia with asymmetries of regional cerebral cortical surface area, thickness and subcortical volume measures in 32,256 participants from the UK Biobank. PRS for the two disorders were positively correlated $\left(r=0.08, p=7.13 \times 10^{-50}\right)$, and both were higher in females compared to males, consistent with biased participation against higher-risk males. Each PRS was associated with multivariate brain asymmetry after adjusting for sex, ASD PRS $r=0.03, p=2.17 \times 10^{-9}$, schizophrenia PRS $r=0.04, p=2.61$ $\times 10^{-11}$, but the multivariate patterns were mostly distinct for the two PRS, and neither resembled average sex differences. Annotation based on meta-analyzed functional imaging data showed that both PRS were associated with asymmetries of regions important for language and executive functions, consistent with behavioural associations that arose in phenome-wide association analysis. Overall, the results indicate that distinct patterns of subtly altered brain asymmetry may be functionally relevant manifestations of polygenic risk for ASD and schizophrenia, but do not support brain masculinization or feminization in their etiologies.

Grant Support: This research was funded by the Max Planck Society (Germany).

\section{Relationship of Monoamine Oxidase a Gene Polymorphisms with Personality Traits}

\author{
E. Y. Shabalina ${ }^{1}$, V. O. Kimeeva ${ }^{2}$, E. Y. Skorova ${ }^{1}$, V. A. Skobeeva ${ }^{1,2}$, \\ E. R. Chertkova ${ }^{3}$, O. V. Parshikova ${ }^{2}$, E. V. Petersen ${ }^{1}$ \\ ${ }^{1}$ Moscow Institute of Physics and Technology (National Research \\ University), Moscow, Russia \\ ${ }^{2}$ Lomonosov Moscow State University, Moscow, Russia \\ ${ }^{3}$ Pirogov Russian National Research Medical University, Moscow, \\ Russia
}

Keywords Behavior genetics, Molecular Genetics, VNTR

This paper investigates the relationship of MAOA gene expression with personality traits. It has already been shown that low expression of MAOA ( 3 and 3.5 repeats of VNTR) is associated with a tendency to violence and mental problems [1]. Two samples of responders (1st sample $-400,17-26$ years, $65 \%$ female, 2 nd sample-380, 15-58 years, $41 \%$ female) filled out Big Five questionnaire [2] and 
HEXACO-PI-R [3]. Respondents' DNA was subjected to fragment analysis by QIAxcel Advanced system. The results were analyzed using IBM Statistics and STATISTICA programs with Pearson correlation, cluster and factor analysis. Higher MAOA expression (4 VNTR repeats) was associated with lower manifestation of integrity compared to lower levels (3 repeats). Correlation between neuroticism and MAOA gene expression was detected only in group of male respondents. Young males with 3 repeats had higher rates of emotional instability compared to carriers of 4 repeats. These results may indicate different effects of MAOA gene expression on personality traits throughout ontogenesis.

\section{References}

1. Kim-Cohen J. et al. MAOA, maltreatment, and gene-environment interaction predicting children's mental health: new evidence and a meta-analysis //Molecular psychiatry. - 2006. - T. 11. - №. 10. - C. 903-913.

2. Egorova M.S., Parshikova O.V. Validation of the Short Portrait Big Five Questionnaire (BF-10). // Psychological Studies, 2016, 9, 45, p. 9. http://psystudy.ru (in Russian, abstr. in English).

3. Egorova M.S., Parshikova O.V., Mitina O.V. Structure of the Russian variant of the six-factor HEXACO personality inventory // Issues of Psychology, 5, 33-49. (in Russian)

\section{The Differential Relations Between ADHD and Reading Achievement: A Quantile Genetic Approach}

Jeffrey Shero ${ }^{1}$, Jessica A. R. Logan ${ }^{3}$, Stephen A. Petrill ${ }^{4}$,

Erik Willcutt ${ }^{5}$, \& Sara A. Hart ${ }^{1,2}$

${ }^{1}$ Department of Psychology, Florida State University, Tallahassee, Florida

${ }^{2}$ Florida Center for Reading Research, Tallahassee, Florida

${ }^{3}$ Department of Educational Studies, The Ohio State University, Columbus, Ohio

${ }^{4}$ Department of Psychology, The Ohio State University, Columbus, Ohio

${ }^{5}$ Department of Psychology, University of Colorado Boulder, Boulder, Colorado

Keywords ADHD, Reading, Bivariate analysis, Quantile analysis, Quantitative methods

This paper extends the understanding of the relation between ADHD and reading comprehension, through examining how this relation differs depending on the quantile an individual falls in for each. Samples from three twin projects around the United States were used (Florida Twin Project, Colorado component of International Longitudinal Twin Study of Early Reading Development, \& Western Reserve Reading and Math Projects). Phenotypic analysis using quantile regression showed relations between ADHD related behaviors and reading comprehension to be stronger in the lower quantiles of reading comprehension in two of three samples. A new method was developed extending this analysis into the bivariate genetic space. Results of this quantile genetic analysis revealed that overlapping common environmental influences accounted for a larger proportion of variance in the lower quantiles of these variables in two of three samples. Finally, in all three samples the phenotypic relation was strongest when shared environmental influences accounted for a larger proportion of the overall variance.

Grant Support: This project was supporting by funding from the Eunice Kennedy Shriver National Institute of Child Health and
Human Development grants P50HD052120, R01HD095193, R01HD038075, R01HD68728, P50HD27802, and R01HD38526.

\section{Neighborhood Features Moderate the Etiology of Children's Social Information Processing}

Elizabeth A. Shewark ${ }^{1}$, Kelly L. Klump ${ }^{1}$, \& S. Alexandra Burt ${ }^{1}$ ${ }^{1}$ Department of Psychology, Michigan State University, East Lansing, Michigan, USA

Keywords Child Social Information Processing, Neighborhood Social Processes, Neighborhood Structural Characteristics

The neighborhood is a key context in which children learn to navigate social situations. While much of this learning leads to adaptive outcomes, some children develop aggressive response styles. Researchers in this area have emphasized the need to consider the potential contributions of multiple neighborhood features (e.g., social processes and structural characteristics), but have largely overlooked the ways in which children's individual-level characteristics might moderate neighborhood effects. As a consequence, little progress has been made in identifying the specific mechanisms through which neighborhood influences children's perception of social situations. We examined 847 6-11 year old twin pairs oversampled for disadvantage from the Twin Study of Behavioral and Emotional Development in Children (TBED-C), and a yoked sample of 1880 randomly-sampled neighbors who reported on neighborhood social cohesion, informal social control, and extent of problems in their neighborhood. We evaluated these neighborhood informant reports as etiologic moderators of children's hostile attributions and expectations for aggressive behavior (i.e., positive outcomes, peer approval, and retaliation). We found no evidence of etiologic moderation of children's expectations of aggressive behavior. However, we found that extent of neighborhood problems moderated nonshared environmental influences on children's hostile attributions such that as neighborhood problems decreased, the amount of variance accounted for by nonshared environmental factors increased. These results suggest that twins who resided in more advantaged neighborhoods differed more in their hostile attributions. Our next steps will be to test various definitions of neighborhood, and to test more traditional conceptualizations of neighborhood disadvantage as assessed using Census data.

Grant Support: F32 HD098780, R01-HD093334

\section{Does Depression Lead to Criminal Behavior? A Sibling Comparison Design Using the NLSY}

Emma E. Sims ${ }^{1}$, Jonathan D Trattner ${ }^{2}$, S. Mason Garrison ${ }^{1}$

${ }^{1}$ Department of Psychology, Wake Forest University, Winston Salem, NC USA

${ }^{2}$ Department of Interdisciplinary Studies, Wake Forest University Winston-Salem, North Carolina, USA

Keywords Depression, Criminal behavior, Justice-involved youth, Delinquency, Sibling comparison

Relative to the general population, adolescents with psychiatric disorders such as major depression disorder are incarcerated (and reincarcerated) at higher rates (McDougall et al., 2013). Current research is mixed whether this association is a cause, consequence, or 
familial confound. For example, correctional facility time leads to more depressive symptoms (Fazel et al., 2008), yet depression is associated with antisocial behaviors (e.g., delinquency; Ozkan et al., 2019). Moreover, most studies have failed to incorporate genetic-andenvironmental confounding. Therefore, we employed the discordant kinship model to see whether compared siblings. We examined the direction and timing of the relationship between criminal behavior and depression, using sibling pairs from the National Longitudinal Surveys of Youth 1979 - a nationally representative study. This allowed us to control for within- and between-family variance, reducing plausible confounds in analyzing the causal relationship between depression and delinquency. After controlling for familial confounds, we failed to find a causal link between depression and delinquency.

\section{References}

Fazel, S., Doll, H., \& Långström, N. (2008). Mental Disorders Among Adolescents in Juvenile Detention and Correctional Facilities: A Systematic Review and Metaregression Analysis of 25 Surveys. Journal of the American Academy of Child \& Adolescent Psychiatry McDougall, A., Campbell, M. A., \& Santor, D. A. (2013). Institutional offense patterns in adolescent offenders: The role of antisocial and mental health indicators. Youth Violence and Juvenile Justice Ozkan, T., Rocque, M., \& Posick, C. (2019). Reconsidering the link between depression and crime: A longitudinal assessment. Criminal Justice and Behavior

\section{Longitudinal methylome-wide analysis in patients undergoing Electroconvulsive Therapy (ECT)}

Lea Sirignano ${ }^{1}$, Josef Frank ${ }^{1}$, Laura Kranaster ${ }^{2}$,

Stephanie H. Witt ${ }^{1}$, Fabian Streit ${ }^{1}$, Lea Zillich ${ }^{1}$, Alexander Sartorius ${ }^{2}$, Marcella Rietschel ${ }^{1}$, Jerome C. Foo ${ }^{1}$

${ }^{1}$ Department of Genetic Epidemiology in Psychiatry, Central Institute of Mental Health, Medical Faculty Mannheim, University of Heidelberg, Mannheim, Germany

${ }^{2}$ Department of Psychiatry and Psychotherapy, Central Institute of Mental Health, Medical Faculty Mannheim, University of Heidelberg, Mannheim, Germany

Keywords Electroconvulsive Therapy (ECT), DNA methylation, Epigenetics, Antidepressant response

Electroconvulsive therapy (ECT) is the treatment of choice for severe and treatment-resistant depression and considered as most effective for this subgroup of patients (Kellner et al, 2020). The molecular mechanisms underlying its effects are largely unknown. As antidepressant treatment has been reported to be associated with epigenetic modifications (Webb et al, 2020); we hypothesized that a strong intervention such as ECT leads to pronounced changes in epigenetic levels. In this study, we analyzed epigenome-wide DNA methylation levels of 34 severely depressed patients before and after ECT treatment using the Illumina Infinium Methylation EPIC BeadChip. Epigenome-wide methylation analysis revealed a significant differential methylated $\mathrm{CpG}$ site in $T N K S$ to be associated with response to ECT. Two differential methylated regions on chromosomes 8 and 20 were associated with a reduction in depressive symptoms. Pathway anaylsis yielded no significant pathway related to ECT response. Our findings show that ECT introduces change in DNA methylation levels and could therefore be considered a promising model to study underlying mechanisms in depression and treatment response. To confirm potentially important results larger sample sizes and replication in other cohorts are needed. To this end, we also present a more comprehensive assessment framework for the ongoing phase 2 of the investigation, including additional phenotypic and epigenetic assessments throughout the course of ECT treatment.

\section{References}

Kellner CH, Obbels J, Sienaert P. When to consider electroconvulsive therapy (ECT). Acta Psychiatr Scand. 2020;141(4):304-315.

Webb LM, Phillips KE, Ho MC, Veldic M, Blacker CJ. The Relationship between DNA Methylation and Antidepressant Medications: A Systematic Review. Int J Mol Sci. 2020;21(3).

Grant Support: This work was supported by the German Research Foundation [DFG; grant FOR2107; RI908/11-2 and WI3429/3-2], the German Federal Ministry of Education and Research (BMBF) through the Integrated Network IntegraMent, under the auspices of the e:Med Programme [01ZX1314G; 01ZX1614G] through grants 01EE1406C, 01EE1409C, Target-OXY [031L0190A] and through ERA-NET NEURON, "SynSchiz-Linking synaptic dysfunction to disease mechanisms in schizophrenia-a multilevel investigation" [01EW1810], through ERA-NET NEURON "Impact of Early life MetaBolic and psychosocial strEss on susceptibility to mental Disorders; from converging epigenetic signatures to novel targets for therapeutic intervention" [01EW1904], and by a grant of the DietmarHopp Foundation. All other authors report no biomedical financial interests or potential conflicts of interest.

\section{Predicting Disordered Gambling Across Adolescence and Young Adulthood from Polygenic Contributions to Big 5 Personality Traits in a UK Birth Cohort}

Kellyn M. Spychala, Ian R. Gizer, Christal N. Davis, Genevieve F. Dash, Thomas M. Piasecki, and Wendy S. Slutske ${ }^{1}$ Department of Psychological Sciences, University of Missouri, Columbia, Missouri, USA

Keywords Disordered gambling, Big 5 personality traits, ALSPAC, Polygenic risk score

Phenotypic associations between disordered gambling (DG) and the Big 5 personality traits have been demonstrated throughout the literature. However, only one twin study to date has examined these genetic associations. The aim of the present study is to examine the genetic associations between the Big 5 personality traits and disordered gambling using multiple polygenic risk score (PRS) approaches, (i.e., pruning and thresholding $(\mathrm{P}+\mathrm{T})$ and PRS-CS). Summary results from a genome-wide association study (GWAS) of the Big 5 personality traits were used as weights for the creation of the PRSs. PRSs were created in 4,729 unrelated children of European ancestry from the Avon Longitudinal Study of Parents and Children (ALSPAC) study. The resulting PRSs were used to predict past-year assessment of DG using the Problem Gambling Severity Index (PGSI) and lifetime assessment of DSM-IV pathological gambling symptoms (DPG) across the ages of 17, 20, and 24. PRSs for agreeableness negatively predicted PGSI scores $(\beta s=-0.25$ to -0.15 ; $P s=$ 0.002-0.009) and PRSs for neuroticism positively predicted PGSI scores $(\beta s=0.11-0.15 ; P s=0.000003-0.001)$ using both PRS methods. PRSs for agreeableness also negatively predicted DPG using the $\mathrm{P}+\mathrm{T}$ method $(\beta=-0.20 ; P=0.0004)$. Finally, the neuroticism PRS showed positive main effects when predicting DPG using PRSCS $(\beta=0.14 ; P=0.009)$ and an interaction with age when using the $\mathrm{P}+\mathrm{T}$ method $(\beta s=0.18-0.29 ; P s=0.002-0.009)$. Findings suggest 
polygenic contributions to low agreeableness and high neuroticism are significantly associated with lifetime and past year measures of DG and these associations are relatively consistent across different PRS approaches.

Grant Support: This research was specifically funded by the Responsible Gambling Fund (A. Emond, PI) and Gamble Aware (A. Emond, PI), and a Center of Excellence award (W. Slutske, PI) from the International Center for Responsible Gaming, and a grant from the National Institute on Alcohol Abuse and Alcoholism: T32AA-013526 (K. Sher, PI).

\section{What Drives the Relation Between Cognitive Ability, Conscientiousness, Self-perceived Ability and School Grades?}

\author{
Alexandra Starr ${ }^{1}$, Rainer Riemann ${ }^{1}$ \\ ${ }^{1}$ Department of Psychology, Bielefeld University, Bielefeld, Germany
}

Keywords Twin family study, School performance, Self-perceived ability, Adolescence, TwinLife

The overall level of education has been increasing worldwide over the last decades, still interindividual differences in learning are a topic of interest regarding life outcomes in adulthood. Phenotypic research identified that personal characteristics such as cognitive ability (IQ), conscientiousness $(\mathrm{CON})$ and self-perceived ability (SPA) predict differences in school grades. In educational research, origins of SPA have long been assumed to be environmental, however substantial genetic contributions have been detected in behavioral genetic analyses. The object of this study is to investigate the common etiology of these three predictors and their association with school grades as well as how genetic and environmental contributions differ between age groups and school subjects. The sample consists of over 2000 twin pairs (aged 11 and 17) and their siblings participating in the German TwinLife study. Using a multivariate twin-sib design, we analyze common genetic and environmental effects on IQ, CON, SPA and school grades in math and German in two age groups. Results confirm genetic effects for all three predictors of up to $55 \%$ as well as (non-) shared environmental effects. Multivariate analyses show that the association with school grades in math and German as well as the relation between CA, CON and SPA is largely due to common genetic effects. In addition, non-shared environmental effects matter across age groups and domains, while shared twin specific environmental effects are only relevant for the relation between SPA and school grades, however effects are small.

Grant Support: The TwinLife study is supported by a grant from the German Research Foundation (DFG) awarded to Rainer Riemann (RI 595/8-1), Martin Diewald (DI 759/111) and Frank M. Spinath (SP 610/6-1).

\section{Cortical and Subcortical Grey Matter Micro-structure is Associated with Polygenic Risk for Schizophrenia}

Eva - Maria Stauffer ${ }^{1}$, Richard A.I. Bethlehem ${ }^{1 *}$, Varun Warrier ${ }^{1 *}$, Graham K. Murray ${ }^{1,2,3}$, Rafael Romero-Garcia ${ }^{1}$, Jakob Seidlitz ${ }^{4,5}$, Edward T. Bullmore ${ }^{1}$

${ }^{1}$ University of Cambridge, Department of Psychiatry, Cambridge Biomedical Campus, CB2 0SZ, UK
${ }^{2}$ Cambridgeshire and Peterborough NHS Trust, Elizabeth House, Fulbourn Hospital, Cambridge CB21 5EE, UK

${ }^{3}$ Institute for Molecular Bioscience, University of Queensland, St Lucia 4072, Australia

${ }^{4}$ Department of Child and Adolescent Psychiatry and Behavioral Science, Children's Hospital of Philadelphia, Philadelphia, PA, USA ${ }^{5}$ Department of Psychiatry, University of Pennsylvania, Philadelphia, PA, USA

*Equal contribution

Keywords Polygenic risk score, Schizophrenia, Structural MRI, Subcortex, Cortex

The current evidence linking structural brain MRI markers to polygenic risk for schizophrenia is inconsistent. We analysed multimodal MRI and genotype data on $\mathrm{N} \sim 30,000 \mathrm{UK}$ Biobank participants to perform a phenome-wide association study using polygenic risk scores for schizophrenia (PRS) and nine cortical and five subcortical MRI phenotypes. We measured PRS for schizophrenia based on the largest genome-wide association (GWAS) dataset in a large sample from the UK Biobank who had multiple micro- and macro-structural MRI metrics measured at 180 cortical areas and seven subcortical structures. We report significant associations between PRS and regional neurite density index, fractional anisotropy, orientation dispersion index, local gyrification index, volume, surface area and intrinsic curvature. Micro-structural phenotypes derived from diffusion tensor imaging data were more robustly associated with schizophrenia PRS than macro-structural phenotypes. PRS was significantly associated with reduced neurite density index (NDI), a measure of the density of myelinated axons and dendrites, at global brain scale, at 149 cortical regions, and five subcortical structures. Genetic effects on multiple MRI phenotypes were co-located in temporal, cingulate and prefrontal cortical areas, insula, and hippocampus. These findings are in line with reported reductions in NDI in schizophrenia patients, post-mortem studies showing abnormalities in neurite structure and GWAS results pointing towards risk genes implicated in functional processes, such as synaptic organisation and transmission. Taken together, these results suggest that the genetic risk for schizophrenia is associated with cortical and subcortical brain structure.

Grant Support: E.-M.S is supported by a $\mathrm{PhD}$ studentship awarded by the Friends of Peterhouse. This research was co-funded by the National Institute of Health Research (NIHR) Cambridge Biomedical Research Centre and a Marmaduke Sheild grant to R.A.I.B. and V.W. R.R.G was funded by a Guarantors of Brain Fellowship. R.A.I.B is supported by a British Academy Post-Doctoral fellowship and the Autism Research Trust.

\section{The Analysis of the Polymorphic Variant rs10494561 of the Gene NMNAT2 in the Development of Spatial Cognition of the Individual}

\footnotetext{
${ }^{* 1}$ Takhirova Z.R., ${ }^{1,2}$ Ismatullina V.I., ${ }^{1}$ Adamovich T.V., ${ }^{1,3}$ Kazantseva A.V., ${ }^{1,3}$ Enikeeva R.F., ${ }^{1,2}$ Lobaskova M.M., Soldatova E.L., ${ }^{3,4}$ Khusnutdinova E.K., ${ }^{2,4}$ Malykh S.B.

${ }^{1}$ Russian Academy of Education, Moscow, Russian Federation

${ }^{2}$ Psychological Institute, Russian Academy of Education, Moscow, Russian Federation

${ }^{3}$ Institute of Biochemistry and Genetics of Ufa Federal Research Centre of the Russian Academy of Sciences, Ufa, Russian Federation ${ }^{4}$ Moscow State University, Moscow, Russian Federation

${ }^{5}$ Saint-Petersburg State University, Saint-Petersburg, Russian Federation
} 
Keywords Spatial ability, Cognition, Gene, Polymorphic Variant, Intellect

Spatial abilities-evolutionarily adaptive cognitive skills, which act as a reliable predictor of an individual's academic achievement in scientific fields related to STEM. Genetically informative studies have shown that spatial abilities are moderately inherited (30-50\%). In the present study, we undertook the analysis of the polymorphic variant rs10494561 in the gene NMNAT2 with the development of spatial intelligence in 312 students aged 17-34 years of different sex and professional direction by using KASP technology (LGC, Biosearch Technologies). The gene NMNAT2 is involved in key processes in maintaining neuronal activity, protecting the nervous system from stress (Ali et al., 2016). Analysis of the association of the polymorphic locus was carried out in correlation with: sex, professional sphere and psychological correlates (4 tests for space: mechanical reasoning; paper folding; pattern assembly; share rotation, academic performance, test of Raven). Preliminary statistical evaluation with the introduction of the distance of Mahalonobis (>95\%) revealed 16 respondents with deviations at data of testing, and therefore they were removed from the study. So that, the group included 296 people $(60$ men, 236 women). The results of genotyping of the polymorphic variant were evaluated by using the software PLINK 1.9 package. However, we not observed statistically significant results $(\mathrm{p}>0.05)$, which allows us to conclude that the $r s 10494561$ in the gene NMNAT2 is not associated with the development of spatial intelligence in our cohort. Perhaps this is due to the small sample size.

Grant Support: The present study was supported by the Russian Science Foundation (Project No. 17-78-30028).

\section{The Analysis of the Polymorphic Variant rs2285351 in the Gene of IFT122 in the Development of Spatial Abilities of Students}

\author{
*1 Takhirova Z.R., ${ }^{1,2}$ Ismatullina V.I., ${ }^{1}$ Adamovich T.V., ${ }^{1,3}$ \\ Kazantseva A.V., ${ }^{1,3}$ Enikeeva R.F., ${ }^{1,2}$ Lobaskova M.M., \\ ${ }^{3,4}$ Khusnutdinova E.K., ${ }^{2,4}$ Malykh S.B. \\ ${ }^{1}$ Russian Academy of Education, Moscow, Russian Federation \\ ${ }^{2}$ Psychological Institute, Russian Academy of Education, Moscow, \\ Russian Federation \\ ${ }^{3}$ Institute of Biochemistry and Genetics of Ufa Federal Research \\ Centre of the Russian Academy of Sciences, Ufa, Russian Federation \\ ${ }^{4}$ Moscow State University, Moscow, Russian Federation
}

Keywords Spatial ability, Cognition, Gene, Polymorphic variant, Intellect

Spatial abilities play a significant role in a person's success, they are a reliable predictor of his academic achievements in scientific fields related to STEM. The conducting of massive longitudinal screenings are show $\sim 69 \%$ of all individual differences are due to the participation of various genetic factors (Rimfeld et al., 2017). We searched for the association of the polymorphic variant $r s 2285351$ in the gene IFT122 with the development of the spatial abilities of 312 young people different sex, aged 17-34, studying in higher educational institutions with using KASP technology (LGC, Biosearch Technologies). The analysis of the association was carried out in such indicators as: sex, professional sphere and psychological correlates (4 tests for space: mechanical reasoning; paper folding; pattern assembly; share rotation, academic performance, test of Raven). The statistical evaluation revealed deviations in 16 respondents on data of testing in the distance of Mahalanobis ( $>95 \%)$ and therefore they were removed from the study. So that the group consist of 296 people ( 60 men, 236 women). For evaluate the results of genotyping, we using the software PLINK 1.9. During the analysis, we found statistically significant differences in comparing individuals of the technical and socio-humanitarian spheres ( $\beta=1.441, \mathrm{p}=0.0387)$, which indicates the involvement of $r s 2285351$ of the gene IFT122 in the development of spatial intelligence in our group. It is also known that $r s 2285351$ is associated with the development of the spatial orientation trait (Bi et al., 2017).

Grant Support: The present study was supported by the Russian Science Foundation (Project No. 17-78-30028).

\section{The Role of Genetics and the Environment on the Relationship Between Weight Related Indicators and Common Mental Health: Results from Two Twin Studies}

Ellen J. Thompson ${ }^{1}$

${ }^{1}$ Social, Genetic \& Developmental Psychiatry Centre, Institute of Psychiatry, Psychology \& Neuroscience, King's College London, UK and Department of Twin Research and Genetic Epidemiology, School of Life Course Sciences, King's College London

Keywords Twin modelling, Weight, Emotional symptoms

This project aims to (i) investigate the directionality and etiology between BMI and depression and (ii) explore the magnitude of genetic and environmental influences on different types of adiposity indicators, anxiety, depression, and their associations. Data was draw from the Twins Early Development Study (TEDS) and the TwinsUK registry were used. Self-report depressive symptoms and self-report height and weight at ages 12,16 and 21 years were used from TEDS and self-report emotional symptoms and measured height, weight and adiposity indicators were used from TwinsUK. A genetically informative crosslagged model (first described by Burt et al., 2006) was used to estimate the phenotypic longitudinal effect and etiology between measures in 4833 twin pairs from TEDS, whilst adjusting for the concurrent genetic and environmental associations between them. A multivariate ACE model was used to estimate the genetic and environmental influences on the covariance between anxiety, depression, adiposity indicators and body mass index in TwinsUK. Results from TEDS indicate a bidirectional phenotypic association between BMI and depression between ages 12 to 16 , but not between ages 16 to 21 years. Results from TwinsUK show a small but significant genetic correlation between adiposity indicators and depression. These findings will be discussed in light of the growing literature on the obesity and mental health.

\section{References}

1. Burt SA, McGue M, Iacono WG, Krueger RF. Differential Parent-Child Relationships and Adolescent Externalizing Symptoms: Cross-Lagged Analyses Within a Monozygotic Twin Differences Design. Dev Psychol. 2006;42(6):1289-1298. https://doi.org/10.1037/0012-1649.42.6.1289

Grant Support: EJT acknowledges funding from the Wellcome Trust (WT212904/Z/18/Z). 


\section{Principal Components Analysis Corrects Collider Bias in Polygenic Risk Score Effect Size Estimation}

Nathaniel S. Thomas, M.S. ${ }^{1}$, Peter Barr, Ph.D ${ }^{1}$, Fazil Aliev, Ph.D. ${ }^{1}$, Mallory Stephenson, M.S. ${ }^{3}$, Sally I. Kuo, Ph.D. ${ }^{1}$,

Danielle M. Dick, Ph.D. ${ }^{1,2}$, Jessica E. Salvatore, Ph.D. ${ }^{1,3}$

${ }^{1}$ Department of Psychology, Virginia Commonwealth University, Box 842018, Richmond, VA 23284-2018

${ }^{2}$ Department of Human \& Molecular Genetics, Virginia Commonwealth University, Box 980033, Richmond, VA 23298-0033

${ }^{3}$ Virginia Institute for Psychiatric and Behavioral Genetics, Box 980126, Richmond, VA 23298-0126

Keywords Polygenic Risk Scores, Collider Bias, Principal Components Analysis

Genome-wide polygenic scoring has emerged as a way to predict psychiatric and behavioral outcomes and identify environments that moderate the expression of genetic predispositions. An increasing number of studies demonstrate that the inclusion of heritable environments as covariates may bias the effects of polygenic risk scores (PRS) when the environmental covariates are influenced by unmeasured confounding variables, an example of collider bias. We tested the hypothesis that the inclusion of the principal components of observed confounders as covariates may correct for the effect of unmeasured confounders. We conducted a simulation study to test principal components analysis (PCA) as a correction for collider bias. Data were sampled from a model that tested different values for the effect of the polygenic risk score on the heritable environment, the correlation structure of the unmeasured confounding data, and the proportion of the confounding data that is used to construct the principal components. Other model parameters were fixed across all simulation iterations. Modeling the first PC of observed confounders as a covariate recovers the PRS effect size estimate under reasonable assumptions about the proportion of the confounding data that is measured or the correlation structure of the confounding data. Required assumptions become stricter as the association between PRS and the heritable environment (and the magnitude of bias) increases. Inclusion of the first PC of observed confounders may improve the accuracy of PRS effect size estimation when heritable environments are included in the model as covariates. Future directions include application of this method in observed data.

\section{Grant Support: R01AA028064}

\section{Dissecting the Association Between Trauma, Polygenic Risk and Depression: Specific Types of Trauma and Individual Symptoms}

Jackson G. Thorp ${ }^{1,2}$, Zachary F. Gerring ${ }^{1}$, Lucia Colodro Conde ${ }^{3}$, Enda M. Byrne ${ }^{4,5}$, Sarah E. Medland ${ }^{3}$, Christel M. Middeldorp ${ }^{5,6}$ Eske M. Derks ${ }^{1}$

${ }^{1}$ Translational Neurogenomics, QIMR Berghofer Medical Research Institute, Brisbane, Australia

${ }^{2}$ Faculty of Medicine, University of Queensland, Brisbane, Australia ${ }^{3}$ Psychiatric Genetics, QIMR Berghofer Medical Research Institute, Brisbane, Australia

${ }^{4}$ Institute for Molecular Bioscience, University of Queensland, Brisbane, Australia

${ }^{5}$ Child Health Research Centre, University of Queensland, Brisbane, Australia
${ }^{6}$ Child and Youth Mental Health Service, Children's Health Queensland Hospital and Health Service, Brisbane, Australia

Keywords Trauma Depression Symptoms PRS Interactions

Experiencing trauma in either childhood or adulthood is associated with an increased risk for major depression. The effect of different types of traumatic experiences and their interaction with polygenic risk has not been extensively investigated. Here, we investigate the unique effects of ten different types of trauma, including childhood abuse, domestic / intimate partner abuse, and traumatic events, plus their interaction with depression polygenic risk scores (PRS) on overall depression and individual depressive symptoms. We use data from 128,906 individuals in the population-based UK Biobank cohort. Nine out of the ten traumas were uniquely associated with depression. Emotional traumas had the strongest association, and effects were different between sexes: intimate partner emotional abuse had a larger effect on depression in males, while childhood emotional neglect had a larger effect in females. A positive, additive PRS-by-childhood emotional neglect interaction effect on depression was observed, but no other PRS-by-trauma interactions were observed for the other types of trauma. Additionally, our results suggest that trauma is associated with specific patterns of depression symptoms. Broadly, trauma was associated with experiencing atypical neurovegetative symptoms of depression. Overall, our results suggest a complex relationship between trauma, genetic risk and depression, with particular traumatic experiences interacting with genetic risk and leading to different symptomatology.

\section{Association of ADHD Polygenic Risk Score with ADHD and Learning Disorder, Depression, Body Mass Index and MCI}

Rosemary Toomey ${ }^{1}$, Ruth McKenzie ${ }^{1}$, Carol E. Franz ${ }^{2}$, William S. Kremen ${ }^{2}$, Michael J. Lyons ${ }^{1}$

${ }^{1}$ Department of Psychological and Brain Sciences, Boston University, Boston, Massachusetts, USA

${ }^{2}$ Department of Psychiatry and Center for Behavior Genetics of Aging, University of California San Diego, La Jolla, California, USA

Keywords ADHD, Polygenic risk score, MCI, Attention

Attention deficit-hyperactivity disorder (ADHD) symptoms often persist in adulthood and resemble some symptoms of mild cognitive impairment (MCI), as well as constitute a risk factor for dementia. It is unclear if $\mathrm{ADHD}$ is misdiagnosed as MCI due to symptom overlap or if there is a genuine association between the two. ADHD is also more common in men than women. Subjects were 886 individual male twins from the Vietnam Era Twin Study of Aging assessed across three time points (mean ages of 56, 62, and 68). These individuals represent those in the upper and lower tertiles of an ADHD polygenic risk score (PRS) $(n=443$ each). Through linear mixed models controlling for twin status, age, and ethnicity, we evaluated the association of the PRS groups with ADHD itself assessed via selfreport at wave 2 and other phenotypes often associated with ADHD: concurrently assessed learning disorders and body mass index [BMI], major depressive disorder assessed in their forties, as well as with MCI diagnosis at all three waves. The PRS was significantly associated with ADHD $(\mathrm{p}=.031)$ and inattention $(\mathrm{p}=.039)$, but not learning disorder, depression, or BMI. The ADHD PRS was significantly associated with MCI only at wave $3(\mathrm{p}=.036)$. The association between ADHD and MCI may not simply reflect symptom overlap. Genetic liability for ADHD, validated by association with phenotypic 
ADHD, was also associated with the phenotypic presentation of MCI at mean age 68, but not earlier. Further research is warranted to see if this association increases as these men continue to age.

Grant Support: R01 AG050595, R01 AG022381, P01 AG55367, R01 AG060470

\section{Modeling Cross-Generational Polygenic Risk of Depression in the UK Biobank}

\author{
Justin D. Tubbs ${ }^{1}$, Pak C. Sham ${ }^{1,2,3}$ \\ ${ }^{1}$ Department of Psychiatry, The University of Hong Kong, Hong \\ Kong, China \\ ${ }^{2}$ Centre for PanorOmic Sciences, The University of Hong Kong, \\ Hong Kong, China \\ ${ }^{3}$ State Key Laboratory of Brain and Cognitive Sciences, The \\ University of Hong Kong, Hong Kong, China
}

Keywords Depression, Genetic Nurture, Maternal Effect, Polygenic Score, Development

Depression is well known to run in families, with studies typically focusing on the influence of genetic transmission or familial environmental factors, which can be confounded (Jami et al., 2021). Renewed interest has grown in estimating and controlling for genetic nurture, the effects of parental genotypes above and beyond those accounted for by genetic transmission. Leveraging recent methodological advancements (Hwang et al., 2020; Tubbs et al., 2021), we infer SNP genotypes of missing parents in 35,975 individuals from the UK Biobank, subsequently calculating offspring, maternal, and paternal polygenic scores for depression (oPGS, mPGS, pPGS). Our current study uses generalized linear mixed models to estimate the joint and interactive effects of these PGS on offspring depression. Controlling for age, sex, and genetic principal components, we find evidence for a statistically significant interaction between oPGS and mPGS ( $p=0.013)$, as well as between mPGS and pPGS $(p=0.005)$. These interactions indicate that risk for depression is more strongly influenced by offspring genetic risk when maternal genetic risk is low. Results also suggest that although both parents having a high PGS is associated with greater risk of depression in the offspring, both parents having a low PGS is also predictive of greater offspring depression risk. Follow-up analyses indicate that these effects may be specific to females only, and that they may be partially mediated by childhood abuse or feeling loved as a child. Thus, our analysis supports the presence of context-dependent maternal genetic effects on offspring depression and suggests potential environmental mediators.

\section{References}

Jami ES, Hammerschlag AR, Bartels M, Middeldorp, CM. (2021). Parental characteristics and offspring mental health and related outcomes: a systematic review of genetically informative literature. Translational Psychiatry https://doi.org/10.1038/s41398-021-01300-2

Hwang LD, Tubbs JD, Luong J, Lundberg M, Moen G, Sham PC, Cuellar-Partida G, Evans DM (2020). Estimating indirect parental genetic effects on offspring phenotypes using virtual parental genotypes derived from sibling and half sibling pairs. PLoS Genetics. https://doi.org/10.1371/journal.pgen.1009154
Tubbs JD, Hwang LD, Luong J, Evans DM, Sham PC (2021). Modeling Parent-Specific Genetic Nurture in Families with Missing Parental Genotypes: Application to Birthweight and BMI. Behavior Genetics. https://doi.org/10.1007/s10519-020-10040-w

Grant Support: None.

\section{Genetic Association of Nucleus Accumbens 5-HIAA Level and Alcohol Preference Drinking in Quasi-Congenic RQI Mouse Models}

\author{
Csaba Vadasz ${ }^{1,2}$, Beatrix M. Gyetvai ${ }^{3}$ \\ ${ }^{1}$ Laboratory of Neurobehavior Genetics, Nathan S. Kline Institute for \\ Psychiatric Research, 140 Old Orangeburg Rd., 10962, Orangeburg, \\ NY, USA \\ ${ }^{2}$ Department of Psychiatry, New York University Langone Medical \\ Center, New York, NY, USA \\ ${ }^{3}$ Laboratory of Neurobehavior Genetics, Nathan S. Kline Institute for \\ Psychiatric Research, 140 Old Orangeburg Rd., 10962, Orangeburg, \\ NY, USA
}

Keywords Addiction, Alcohol, Congenic, Serotonin, 5-HIAA

Quasi-congenic Recombinant QTL Introgression strains were initially used to identify the first gene ( $\mathrm{Grm} 7$, Glutamate Metabotropic Receptor 7) as accounting for alcohol consumption in a mammalian model. Quasi-congenic mice of the alcohol avoiding C5A3 and the alcohol preferring I5B25A strains were subjected to in vivo microdialysis in the Nucleus Accumbens (NAc) to test the hypothesis that genetic predisposition to high alcohol drinking behavior is associated with hypoactive serotonerg function in the NAc. Neurotransmitter and metabolite contents were analyzed by HPLC in dialysate samples collected in three phases: Baseline, Control (after saline injection, i.p.), and Alcohol (after alcohol injection, $1.5 \mathrm{~g} / \mathrm{kg}$ i.p.). Samples were collected every $20 \mathrm{~min}$ before and after each injection. 5-HIAA levels in the alcohol preferring I5B25A strain were consistently lower in dialysate samples in the course of all three phases. GLM RM ANOVA and Tests of Between-Subjects Effects showed a significant strain difference. Microsatellite DNA marker genotyping of the biallelic strains allowed the identification of differential chromosome regions associated with low 5-HIAA levels and high alcohol drinking. Testing 19 autosomal chromosomes of the C5A3 and I5B25A strains we found 16 differential markers distributed on 5 chromosomes. The results are consistent with accumulated evidence for the involvement of serotonin in reward processing, and support earlier reports of similar association in rats and primates, including Homo sapiens. The data suggest that further genetic selection and construction of congenic lines can lead to the identification of pleiotropic candidate gene(s) affecting both the serotonerg system and alcohol preference drinking behavior.

Grant Support: Development of animal models was supported by The National Institute of Neurological Disorders and Stroke NS19788, The National Institute on Alcohol Abuse and Alcoholism R01 AA11031, and United States Department of Defense (U.S. Army Medical Research and Materiel Command DAMD 17-00-1-0578). 


\section{Measuring 100 Personality Traits and 9 Cognitive Domains at Biobank Scale Under 1 Hour}

\author{
Uku Vainik ${ }^{1,2}$, Mariliis Vaht ${ }^{3,1}$, Rene Mõttus ${ }^{4,1}$ \\ ${ }^{1}$ Institute Psychology, Faculty of Social Sciences, University of Tartu, \\ Tartu, Estonia \\ ${ }^{2}$ Department of Neurology and Neurosurgery, Faculty of Medicine \\ and Health Sciences, , McGill University, Montreal, Canada \\ ${ }^{3}$ Institute of Genomics, Faculty of Science and Technology, Univer- \\ sity of Tartu, Tartu, Estonia \\ ${ }^{4}$ Department of Psychology, University of Edinburgh, Edinburgh, UK
}

Keywords Personality, Cognition, Online, Nuances, Reliability

Genetics of behavioral traits tend to focus on Big Few - broad personality traits and global IQ. Detailed measures can offer at least two times more insight into health and life outcomes. Unfortunately, biobanks rarely use detailed measures and therefore statistical power for gene discovery is low. For quick and detailed personality measurement, we are developing open source personality test One Hundred Nuances of Personality (100NP). Provisionally, its 200 questions will assess 14-22 nuances per each Five-Factor Model domain as well as nuances beyond them. Because of its wide coverage, 100NP also measures the traits of other personality tests, including NEO PI-R, HEXACO, and impulsivity measures. Pilot data from UK $(\mathrm{n} \sim 1000)$ shows that 100NP nuances have excellent testretest reliability $(r=.70-.80)$ and adequate narrow sense heritability $(\mathrm{h} 2=.20)$. For cognition, we hand-picked 9 maximally independent freely available tasks by testmybrain.org. 296 Estonians participated online twice over a week, once using a computer and another time using a handheld device. Device switch hurt test-retest reliability of motor tasks, such as simple reaction time ( $\mathrm{RT}, \mathrm{r}=.30)$, Trails A $(\mathrm{r}=$ $.31)$ and $B(r=.32)$, but not others, even across parallel versions: matrixes: $r=0.49$, digit-symbol coding: $r=0.73$, vocabulary: $r=$ 0.83 ; verbal memory: $r=0.58$, delay discounting: $r=0.85$, choice RT: $r=0.69$. Average completion time was 25 minutes for personality and the same for cognition. The measure set will be used by Estonian Biobank $(N=200,000)$, and likely in several other biobanks. We hope that these detailed open source measures will pave way to genomics of detailed human behaviors.

Grant Support: Estonian Research Council Start-up grant PSG656

\section{The Effects of Demographic-Based Selection Bias on GWAS Results in the UK Biobank}

\author{
Sjoerd van Alten, ${ }^{1,2}$ Benjamin W. Domingue, ${ }^{3}$ Titus Galama, ${ }^{1,2,4,5}$ \\ and Andries Marees ${ }^{1}$ \\ ${ }^{1}$ School of Business and Economics, Vrije Universiteit Amsterdam, \\ Amsterdam, Netherlands \\ ${ }^{2}$ Tinbergen Institute, Amsterdam, Netherlands \\ ${ }^{3}$ Stanford University, Stanford, California, USA \\ ${ }^{4}$ Center for Economic and Social Research and Department of Eco- \\ nomics, University of Southern California, Dornsife, Los Angeles, \\ USA \\ ${ }^{5}$ Erasmus School of Economics, Erasmus University Rotterdam, \\ Rotterdam, Netherlands
}

Keywords Genome wide association studies, Selection bias, UK Biobank

Genome-wide association studies (GWASs) are almost always based on a non-random sample of the underlying population, as obtaining very large sample sizes, rather than ensuring such samples are representative, has been key to their success. Selection bias in estimated genetic associations, including how it varies across traits, is poorly understood. A sample of particular interest is the widely used UK Biobank (UKB). Individuals in the UKB are more likely to be female, higher educated, and older, due to its reliance on volunteering. Because of the need for very large samples, the UKB, by far the largest cohort, is included in almost all large GWASs. Further, UKB's subsample of genotyped siblings (UKBSIB) has become a crucial resource for estimating genetic effects free of environmental confounding. Using nationally representative UK Census microdata as a reference, we document substantial non-random selection into the $\mathrm{UKB}$, and even stronger for UKBSIB. This non-random selection leads to significant selection bias in associations between various demographic and health-related traits when estimated in the UKB. We estimate probabilities of UKB participation for each UKB participant to estimate selection-corrected GWASs for multiple traits using (1) inverse probability weighting and (2) a Heckman two-step correction. We will assess whether selection-corrected GWAS results significantly differ from those of traditional GWASs and investigate which phenotypes are most affected, and why. Our results are useful for understanding whether a particular phenotype is prone to selection bias in GWAS and our correction method provides an alternative when population-representative cohorts are not available.

Grant Support: We gratefully acknowledge financial support from NORFACE DIAL (462-16-100), the National Institute on Aging of the National Institutes of Health (RF1055654 and R56AG058726), and the Dutch National Science Foundation (016.VIDI.185.044).

\section{The Role of Personality in Voluntary Exercise Behaviors: A Mendelian Randomization Study}

Matthijs D van der Zee ${ }^{1}$, Lianne P de Vries ${ }^{1}$, René Pool ${ }^{1}$, Michel G Nivard ${ }^{1}$, Jouke J Hottenga ${ }^{1}$, Erik Ehli ${ }^{2}$, Meike Bartels ${ }^{1}$, Connor V Dolan ${ }^{1}$, Jenny van Dongen ${ }^{1} \&$ Eco J C de Geus ${ }^{1}$

${ }^{1}$ Department of Biological Psychology, Netherlands Twin Registry, Vrije Universiteit, Amsterdam, Netherlands

${ }^{2}$ Avera Institute for Human Genetics, Sioux Falls, South Dakota, United States of America

Keywords Exercise behavior, Personality, Causality, Mendelian randomization

Personality, the individual-level tendencies to show consistent patterns of thoughts and behaviors has long been associated with exercise behavior. The question of causality, however, still remains, as genetic and environmental confounding are difficult to rule out. The use of traditional intervention methods to test causal effects of personality are rather difficult, if not impossible. Here we instead use triangulation across different methods to falsify the causal hypothesis, where failure to falsify across these methods strengthens our confidence in the causal hypothesis. Using a sample from the Netherlands Twin Register $(\mathrm{N}=39,577)$ we first establish the cross-sectional and longitudinal associations between the 5 NEO personality traits and exercise phenotypes. Second, controlling for genetic confounding, we assess the regression of monozygotic intrapair differences in both type of traits. Third, twin-sibling structural equation models are used to assess significant correlation of all latent factors influencing personality and exercise. Finally, to test for causality in the presence of genetic pleiotropy, we used the MR-DoC model. Results varied greatly depending on the personality trait and type of exercise. Strongest support for the causal hypothesis was found for the effect of 
extraversion on total volume of exercise, followed closely by the effects of conscientiousness on total exercise. On the other hand, no support was found for causal effects of neuroticism, agreeableness or openness. These results shed new light on the causality underlying the well-established relationship between personality traits and exercise behavior.

\section{Why the COVID-19 Pandemic is Interesting from a Behavior Genetic Perspective: A Focus on Quality of Life and Self-rated Health}

\author{
Margot P. van de Weijer ${ }^{1,2}$, Lianne P. de Vries ${ }^{1,2}$, Dirk Pelt ${ }^{1,2}$, \\ Floris Huider ${ }^{1,2}$, Matthijs D. van der Zee ${ }^{1,2}$, Lannie Ligthart ${ }^{1,2}$, \\ Gonneke Willemsen ${ }^{1,2}$, Dorret I. Boomsma ${ }^{1,2}$, \\ Eco de Geus ${ }^{1,2}$ \& Meike Bartels ${ }^{1,2}$ \\ ${ }^{1}$ Department of Biological Psychology, Vrije Universiteit Amster- \\ dam, The Netherlands \\ ${ }^{2}$ Amsterdam Public Health Research Institute, Amsterdam, The \\ Netherlands
}

Keywords Covid-19, Quality of life, Self-rated health, Gene-environment interaction, Extended twin-pedigree

Due to lockdown and social distancing measures, the COVID-19 pandemic has not only impacted those who have been infected by the virus, but also those who remain infected. An important question in this regard is how the pandemic has affected people's (mental) health. Moreover, from a behavior genetic perspective, an interesting question is how this large environmental change might have impacted (sources of) individual differences in health measures. We present two (ongoing) projects that focus on the influence of the COVID-19 pandemic on the Dutch population using Netherlands Twin Register data. First, we present a project where we use three sets of analyses to study the impact of the pandemic on self-rated health (SRH). These analyses include (1) comparisons of pre-pandemic and pandemic SRH scores, (2) the impact of age, sex, educational attainment, and chronic illness on pre-pandemic SRH, pandemic SRH and SRH difference scores, and (3) bivariate genetic analyses in a sample of twins to decompose (co)variation in SRH at the two time-points into genetic and environmental sources. Second, we present a project where we use the Mendel software package (Lange et al, 2013) to perform an extended twin-pedigree study of quality of life during and before the pandemic. The projects provide two important messages. First, the projects illustrate how intertwined genetic and environmental influences are, and that heritability is far from a fixed predisposition. Second, they reconfirm the importance of examining individual differences instead of mean effects when studying complex human traits.

\section{References}

Lange K, Papp JC, Sinsheimer JS, Sripracha R, Zhou H, Sobel EM (2013) Mendel: the Swiss army knife of genetic analysis programs. Bioinformatics 29(12):1568-1570

Grant Support: This work is supported by the NWO Corona FastTrack grant (440.20.022), and the European Research Council Consolidator Grant (ERC-2017-COG 771057 WELL-BEING PI Bartels).

\section{Intergenerational Effects on Offspring Criminal Behavior: A Children of Siblings Design}

Steve van de Weijer ${ }^{1}$

${ }^{1}$ Netherlands Institute for the Study of Crime and Law Enforcement (NSCR); Amsterdam, The Netherlands

Keywords Criminal behavior, Intergenerational transmission, Parental divorce, Early motherhood, Children of sibling design

Previous studies have shown that behavior of parents is associated with the criminal behavior of their offspring. Those with criminal or imprisoned parents have been shown to be more likely to become offenders themselves compared to their peers with non-criminal parents, while also parental divorce and early motherhood are risks factor for the development of antisocial and delinquent behavior in offspring. These studies, however, usually do not control for unmeasured confounders and these associations might therefore be spurious. In this study, a children of siblings design was used in which the criminal behavior of children of discordant siblings (i.e., one criminal father and his non-criminal brother) was compared. This model controls (partly) for confounding by genetic and shared environmental factors and therefore gives a better estimate of the causal effects. Using register data of Statistic Netherlands all children born in the Netherlands between 1991 and 2001 were selected for analyses on the population level $(\mathrm{N} \approx 2,100,000)$. Next, the offspring of siblings who were discordant on parental divorce, paternal offending, and maternal age at birth were compared in three separate conditional logistic regression analyses. The results show that the intergenerational associations become considerably smaller or even disappear when a children of sibling design is used compared to when unrelated individuals are compared. This indicates that these associations are at least partly confounded by genetic or shared environmental factors, and that intergenerational associations are overestimated when these confounding factors are not controlled for.

Grant Support: Steve van de Weijer was supported by grant nr. 45116-014 from the Netherlands Organization for Scientific Research (NWO).

\section{Examining Causality of the Association Between Smoking and DNA Methylation}

Jenny van Dongen ${ }^{1}$, Camelia C. Minică $\breve{2}^{2,3}$, Joshua N. Pritikin ${ }^{4,5}$, Gonneke Willemsen ${ }^{1}$, BIOS Consortium ${ }^{6}$, Jouke-Jan Hottenga ${ }^{1}$, René Pool $^{1}$, Benjamin M. Neale ${ }^{2,3,7}$, Conor V. Dolan ${ }^{1}$, Eco J.C. de Geus ${ }^{1}$, Dorret I. Boomsma ${ }^{1}$, Michael C. Neale ${ }^{4,5,8}$

${ }^{1}$ Department of Biological Psychology, Vrije Universiteit, Amsterdam, The Netherlands

${ }^{2}$ Stanley Center for Psychiatric Disease, Broad Institute of MIT and Harvard, Cambridge, MA, 02142, USA

${ }^{3}$ Analytic and Translational Genetics Unit, Center for Genomic Medicine, Massachusetts General Hospital, Boston, MA, USA

${ }^{4}$ The Department of Psychiatry, Virginia Commonwealth University, Richmond, USA

${ }^{5}$ The Virginia Institute for Psychiatric and Behavioral Genetics, Virginia Commonwealth University, Richmond, USA

${ }^{6}$ The Biobank-based Integrative Omics Studies Consortium

${ }^{7}$ Program in Medical and Population Genetics, Broad Institute of MIT and Harvard, Cambridge, MA, USA.

${ }^{8}$ The Department of Human and Molecular Genetics, Virginia Commonwealth University, Richmond, USA

View author publication 
Keywords Causality, smoking, DNA methylation, MR-DoC model, Epigenetics

Previous epigenome-wide association studies (EWAS) have identified widespread robust differences in DNA methylation between smokers and non-smokers. Smoking-associated methylation levels might reflect: causal effects of smoking on methylation; causal effects of DNA methylation on smoking behavior; a genetic predisposition to smoking, epiphenomena of other exposures that correlate with smoking (e.g., alcohol use); or some combination of these processes. The contributions of different mechanisms to the association between smoking and methylation level may also vary across different CpGs. Here we analyze reversibility and bi-directional causal relationships between smoking and DNA methylation, and address the role of genetic pleiotropy. We analyze whole blood genome-wide DNA methylation data (Illumina 450k arrays) from the Biobank-based Integrative Omics Study (BIOS) consortium, and from twin pairs in the Netherlands Twin Register (NTR). We performed EWAS analyses to compare DNA methylation between current, former, and never smokers $(\mathrm{N}=5318$ from the BIOS consortium), and to assess the relationship between DNA methylation and additional smoking phenotypes, including pack years, cigarettes per day, time since quitting smoking, cotinine levels, and nicotine dependence $(\mathrm{N}=577-2786$ from the NTR; the exact N varies with different smoking phenotypes). We also report results of causal analyses, including the Mendelian Randomization-Direction of Causation (MR-DoC) model (Minică et al. 2018).

\section{References}

Minică, Camelia C., Conor V. Dolan, Dorret I. Boomsma, Eco de Geus, and Michael C. Neale. 2018. "Extending Causality Tests with Genetic Instruments: An Integration of Mendelian Randomization with the Classical Twin Design." Behavior Genetics. https://doi.org/ 10.1007/s10519-018-9904-4.

Grant Support: We acknowledge funding from the National Institute on Drug Abuse grant DA049867, the Netherlands Organization for Scientific Research (NWO): Biobanking and Biomolecular Research Infrastructure (BBMRI-NL, NWO 184.033.111) and the BBRMI-NLfinanced BIOS Consortium (NWO 184.021.007), NWO Large Scale infrastructures, X-Omics (184.034.019), and the Royal Netherlands Academy of Science Professor Award (PAH/6635).

\section{Stop Meta-analyzing, Start Instrumenting: Maximizing the Predictive Power of Polygenic Scores}

Hans van Kippersluis ${ }^{1,2}$, Pietro Biroli ${ }^{3}$, Titus J. Galama ${ }^{1,2,4,5}$, Stephanie von Hinke $e^{1,2,6}$, S. Fleur W. Meddens ${ }^{1}$,

Dilnoza Muslimova $^{1,2}$, Rita Pereira ${ }^{1,2}$, Cornelius A. Rietveld ${ }^{1,2,7}$

${ }^{1}$ Erasmus School of Economics, Erasmus University Rotterdam, Rotterdam, The Netherlands

${ }^{2}$ Tinbergen Institute, The Netherlands

${ }^{3}$ Department of Economics, University of Zurich, Zurich, Switzerland ${ }^{4}$ Center for Social and Economic Research, University of Southern California, Los Angeles, United States

${ }^{5}$ School of Business and Economics, VU Amsterdam, Amsterdam, The Netherlands

${ }^{6}$ School of Economics, University of Bristol, Bristol, United Kingdom ${ }^{7}$ Erasmus University Rotterdam Institute for Behavior and Biology, Rotterdam, The Netherlands
Keywords Polygenic score, Instrumental Variables, Meta-analysis, UK Biobank

A polygenic score is constructed using results of finite-sample Genome-Wide Association Studies (GWASs), and it is therefore a noisy approximation of the true latent genetic predisposition to a certain trait. The conventional way of boosting the predictive power of polygenic scores is to increase the GWAS sample size by meta-analyzing GWAS results of multiple cohorts. In this paper we challenge this convention. Through simulations, we show that Instrumental Variable (IV) regression using two polygenic scores based on independent GWAS samples outperforms the typical Ordinary Least Squares (OLS) model employing a single meta-analysis based polygenic score in terms of bias, root mean squared error, and statistical power. We verify the empirical validity of these simulations by predicting educational attainment (EA) and height in a sample of siblings from the UK Biobank. We show that IV regression betweenfamilies approaches the SNP-based heritabilities, while applying IV regression within-families provides a lower bound on the direct genetic effect. IV estimation improves the predictive power of polygenic scores by $12 \%$ (height) to $22 \%$ (EA). Our findings suggest that measurement error is a key explanation for hidden heritability (i.e., the difference between SNP-based and GWAS-based heritability), and that it can be overcome using IV regression. We derive a practical rule of thumb for when IV outperforms OLS: When the correlation between the two polygenic scores used in IV regression is larger than the square root of 10 divided by $N+10$, with $N$ the sample size of the prediction sample.

Grant Support: This research has been conducted using the UK Biobank Resource. The authors also acknowledge funding from NORFACE through the Dynamic of Inequality across the Life Course (DIAL) programme (462-16-100), from the European Research Council (DONNI 851725 and GEPSI 946647), from the National Institute on Aging of the National Institutes of Health (RF1055654 and R56AG058726), and from the Dutch Research Council (016.VIDI.185.044).

\section{The Relationship Between the Predictive Power of Polygenic Scores and the Genetic Ranking of Individuals Across PGS Construction Methods}

Hans van Kippersluis ${ }^{12}$, Stephanie von Hinke ${ }^{123}$, S.Fleur W. Meddens ${ }^{1}$, Dilnoza Muslimova ${ }^{12}$, Rita Dias Pereira ${ }^{12}$, Cornelius A. Rietveld ${ }^{124}$

${ }^{1}$ Erasmus School of Economics, Erasmus University Rotterdam, Rotterdam, The Netherlands

${ }^{2}$ Tinbergen Institute, The Netherlands

${ }^{3}$ School of Economics, University of Bristol, Bristol, United Kingdom

${ }^{4}$ Erasmus University Rotterdam Institute for Behavior and Biology, Rotterdam, The Netherlands

Keywords Polygenic scores, Ranking, Predictive power, Construction methods

Polygenic scores (PGS) are increasingly being used in medicine and social sciences. While their explanatory power, interpretation and limitations are widely discussed, less attention has been devoted to how their construction affects the ranking of individuals. This is particularly important for screening and personalized medicine since polygenic scores are used to target high risk individuals. How many individuals are erroneously left out of screening when a PGS is 
constructed on basis of a different discovery cohort, or by just including genome-wide significant SNPs? Additionally, the rank consistency of polygenic scores may have consequences for geneenvironment interaction research, especially when researchers identify exposure on the basis of PGS percentiles.

In this paper, we use simulations and empirical data to study the effect of (i) GWAS discovery sample selection and (ii) polygenic score construction methods - Plink vs LDPRED and all SNPs versus genome-wide significant SNPs - on PGS rank switching. Our results suggest that different methodologies yield non-negligible rank switching. To explore potential implications of rank switching, we investigate whether PGS construction method can affect personalized cholesterol-lowering medication decisions for individual at risk of coronary artery disease. Additionally, we illustrate that the interaction between various polygenic scores and year-of-birth varies depending on the PGS construction method. Our results therefore reinforce the need for standardized PGS reporting guidelines.

Grant Support: The author acknowledges funding from NORFACE under the Dynamics of Inequality (DIAL) programme (grant number 462-16-100).

\section{Neighborhood Social Processes as Etiologic Moderators of Resilience}

Alexandra Y. Vazquez ${ }^{1}$, Kelly L. Klump ${ }^{1}$, S. Alexandra Burt ${ }^{1}$ ${ }^{1}$ Department of Psychology, Michigan State University, East Lansing, MI, USA

\section{Keywords Neighborhood, Disadvantage, Resilience, G x E}

Despite the known deleterious effects of chronic disadvantage, a large proportion of exposed youth nevertheless demonstrate resiliency (40-60\%; Vanderbilt-Adriance \& Shaw, 2008). Though resilience is influenced by both genetic and environmental influences, no study to date has examined the extent to which neighborhood social processes may impact the heritability of resilience to neighborhood disadvantage. The current study intends to do just this using a sample of child and adolescent twin families $(\mathrm{N}=1072)$ from the Michigan Twins Project who reside in neighborhoods with concentrated disadvantage as determined by the Area Deprivation Index $(>40 \%)$. Resilience will be assessed using the Strengths and Difficulties Questionnaire completed by a caregiver. We will examine three operationalizations of resilience: the absence of maladaptive behaviors or psychopathology, the presence of adaptive behavior, as well a composite of both. After examining the etiology of resilience, we will fit genotype $\mathrm{x}$ environment interaction models to evaluate whether neighborhood social cohesion and informal social control respectively moderate the etiology of resilience. Consistent with a bioecological model, we expect heritability to decrease and environmental influences to increase at lower levels of social cohesion and informal social control. Likewise, at higher levels of social cohesion and informal social control, we expect heritability to increase and environmental influences to decrease.

\section{References}

Vanderbilt-Adriance, E., \& Shaw, D. S. (2008). Conceptualizing and re-evaluating resilience across levels of risk, time, and domains of competence. Clinical Child and Family Psychology Review, 11(1-2), $30-58$.

Grant Support: NSF Graduate Research Fellowship; The MTP is supported via institutional funds from Michigan State University

\section{The Effects of Adolescent Personality Types on Lifestyle and Mortality: A Longitudinal Study of Twins}

Cheyenne E. Vázquez ${ }^{1}$, Matthew R. Jamnik ${ }^{1}$, Lisabeth Fisher DiLalla $^{1}$

${ }^{1}$ Family and Community Medicine, Southern Illinois University School of Medicine, Carbondale, IL

Keywords MMPI, Personality types, Risk-taking, Mortality, Lifestyle behavior

Certain MMPI personality types are related to various forms of serious psychopathology, potentially resulting in lifestyles related to earlier deaths. Specifically, MMPI factors of hypomania (Ma), schizophrenia (Sc), depression (D), and psychopathic deviate (Pd) are frequently associated with agitation, psychopathy, depression and suicidality, and risk-taking behaviors, respectively. We utilized a sample of twins who completed the MMPI in high school during the 1950 's ${ }^{1}$ and are now in their late 70 's to examine whether the co-twin with higher scores on these 4 MMPI scales was more likely to die earlier than their co-twin, and whether these scales were related to risk level of occupations. MMPI data were collected when twins were in high school in the 1950's; current information regarding the professions, hobbies, and (if applicable) deaths of 66 twins were found through Whitepages and obituaries. A series of multilevel binary logistic regressions, using a score for each scale created from each twin's score minus the twin average, showed that the twin with a higher score of Pd was significantly more likely to die first, $p=.014$. There also was a significant correlation between $\mathrm{Pd}$ and occupation risk level, $r=.38, p=.041$, but there was no evidence of shared genes between these. Pd appears to be the most useful MMPI scale for measuring risk-taking behaviors and timing of death, but more obituary data are necessary in order to confirm this result.

References

${ }^{1}$ Gottesman, II (1963) Heritability of personality: A demonstration. Psychological Monographs: General and Applied, 77(9), 1-21

\section{Association of Obsessive-Compulsive Disorder with Substance Misuse: A Genetically Informative Population and Twin Study}

Suvi Virtanen ${ }^{1,2}$, Ralf Kuja-Halkola ${ }^{2}$, Anna Sidorchuk ${ }^{3,4}$, Lorena Fernández de la Cruz ${ }^{3,4}$, Sebastian Lundström ${ }^{5}$, Jaana Suvisaari $^{6}$, Henrik Larsson ${ }^{2,7}$, Paul Lichtenstein ${ }^{2}$, David Mataix-Cols ${ }^{3,4}$, Antti Latvala ${ }^{1,2}$

${ }^{1}$ Institute of Criminology and Legal Policy, University of Helsinki, Helsinki, Finland

${ }^{2}$ Department of Medical Epidemiology and Biostatistics, Karolinska Institutet, Stockholm, Sweden

${ }^{3}$ Centre for Psychiatry Research, Department of Clinical Neuroscience, Karolinska Institutet, Stockholm, Sweden

${ }^{4}$ Stockholm Health Care Services, Region Stockholm, Stockholm, Sweden

${ }^{5}$ Gillberg Neuropsychiatry Centre, University of Gothenburg, Gothenburg, Sweden

${ }^{6}$ Finnish Institute for Health and Welfare, Helsinki, Finland

${ }^{7}$ School of Medical Sciences, Örebro University, Örebro, Sweden

Keywords OCD, Substance use, Twin study, Register-based, Longitudinal 
It remains unclear whether individuals with obsessive-compulsive disorder (OCD) have an elevated risk of substance misuse, and whether OCD and substance misuse share genetic and/or environmental influences. We investigated the association between OCD and substance misuse in two samples. Sample 1 included all individuals born in Sweden 1932-1997 ( $\mathrm{n}=6,304,188)$. OCD was defined as an ICD-10 diagnosis in the patient register, and substance misuse as register-based substance use-related disorder, criminal conviction, or death. The sample was followed-up through the registers in 1997-2013. Sample 2 included participants of the Child and Adolescent Twin Study in Sweden with self-reported information on OCD-symptoms as well as alcohol and drug dependence symptoms at age $18(\mathrm{n}=9231)$. We estimated the contribution of genetic and environmental influences to the covariance between OCD and substance misuse/dependence using data from full siblings and maternal half-siblings in sample 1, and monozygotic and dizygotic twins in sample 2. In sample 1, OCD was associated with an elevated risk of substance misuse (HR 3.84, 95\% CI 3.74-3.95), and in sample 2, OCD-symptoms were associated with increased alcohol $(\beta=0.47$ [0.41-0.55]) and drug $(\beta=0.69$ [0.41-0.98]) dependence symptoms. Anxiety and depressive disorders did not explain the associations. In both samples, the associations between OCD and substance misuse were explained by genetic (56-68\%) and non-shared environmental factors $(32-44 \%)$. Genetic correlations ranged from $0.28(0.24-0.32)$ to $0.31(0.23-0.40)$. OCD was associated with an elevated risk of substance misuse, independently of the primary comorbidities, anxiety and depression. Genetic and non-shared environmental influences explained the association.

Grant Support: This study was supported by research funding from the Academy of Finland, the Swedish Research Council for Health, Working Life and Welfare, and the Swedish Research Council.

\section{The Consortium on Interplay of Genes and Environment Across Multiple Studies (IGEMS): Does Sleep Duration Moderate Genetic and Environmental Contributions to Cognitive Performance?}

Tina T. Vo ${ }^{1}$, Shandell Pahlen ${ }^{1}$, William S. Kremen ${ }^{2}$, Matt Mc Gue ${ }^{3}$, Anna Dahl Aslan ${ }^{4,5,6}$, Marianne Nygaard ${ }^{7}$, Chandra A. Reynolds ${ }^{1}$, for the IGEMS Consortium

${ }^{1}$ Department of Psychology, University of California, Riverside, Riverside, California, USA

${ }^{2}$ Department of Psychiatry, University of California, San Diego, San Diego, California, USA

${ }^{3}$ Department of Psychology, University of Minnesota, Minneapolis, Minnesota, USA

${ }^{4}$ School of Health Sciences, University of Skövde, Sweden

${ }^{5}$ Department of Medical Epidemiology and Biostatistics, Karolinska Institute, Sweden

${ }^{6}$ School of Health and Welfare, Jönköping University, Sweden

${ }^{7}$ Department of Public Health, University of Southern Denmark, Odense, Denmark

Keywords Sleep, Cognitive Aging, Sleep Duration, Moderation, Cognition

The present study tested whether sleep duration moderates the genetic and environmental variances of cognitive performance using twin samples from the Interplay of Genes and Environment Across Multiple Studies (IGEMS) consortium spanning mid- to late-life: Mage = 57.6, range 27 to 91 years, $N=7052$, Female $=43.70 \%, 1525$ complete MZ pairs, 2001 complete DZ pairs. Measures included sleep duration, and four cognitive tests tapping verbal fluency, spatial reasoning, processing speed, and episodic memory (i.e., Animal Naming, Block Design, Symbol Digit, and Word List). Phenotypically, the correlations between sleep duration and cognitive performance were modest $(r=-.01$ to -.10$)$. Twin correlations for sleep duration were $r \mathrm{MZ}=.36, r \mathrm{DZ}=.21$. Extended univariate $\mathrm{ACE}$ moderation models were fitted with sleep duration as the primary moderator, accounting for age moderation. Model comparisons suggested significant overall moderation of ACE components for fluency by sleep duration and $\mathrm{AC}$ components for memory, but no significant moderation of ACE components by sleep for speed. Results were inconclusive for spatial reasoning. For fluency, as sleep duration increased, genetic variance $\left(\mathrm{a}^{2}\right)$ in cognition decreased $\left(\mathrm{a}^{2}\right.$ hours $=.45$, $\mathrm{a}^{2}$ 10hours $\left.=.19\right)$ and shared common environment $\left(\mathrm{c}^{2}\right)$ showed a slight U-shaped pattern $\left(\mathrm{c}^{2}{ }_{4 \text { hours }}=.12, \mathrm{c}^{2}{ }_{7 \text { hours }}=.001, \mathrm{c}^{2}{ }_{10 \text { hours }}=.11\right)$. For memory, as sleep duration increased, $\mathrm{a}^{2}$ decreased $\left(\mathrm{a}^{2}{ }_{4 \text { hours }}=.35\right.$, $\left.\mathrm{a}^{2}{ }_{10 \text { hours }}=.003\right)$ and $\mathrm{c}^{2}$ increased $\left(\mathrm{c}^{2}{ }_{4 \text { hours }}=.003, \mathrm{c}^{2}{ }_{10 \text { hours }}=.37\right)$. For speed, as sleep duration increased, $\mathrm{a}^{2}$ decreased $\left(\mathrm{a}^{2}{ }_{4 \text { hours }}=.63\right.$, $\left.\mathrm{a}^{2}{ }_{10 \text { hours }}=.26\right)$ and $\mathrm{c}^{2}$ increased $\left(\mathrm{c}^{2}{ }_{4 \text { hours }}=.01, \mathrm{c}^{2}{ }_{10 \text { hours }}=.37\right)$. Patterns of unique environmental contributions $\left(\mathrm{e}^{2}\right)$ differed. Notably, genetic variance was highest at short sleep, thus aligning with current literature regarding the association between short sleep and ineffective beta-amyloid clearance which may point to mechanisms underlying poor cognitive performance.

Grant Support: R01 AG059329, R01 AG060470, R01 AG050595, R01 AG022381.

\section{Domain Generality vs. Domain Specificity of Non- ability-Based Confidence in the TwinLife Dataset}

Randi L. $\operatorname{Vogt}^{1} \&$ Daniel A. Briley ${ }^{1}$

${ }^{1}$ Department of Psychology, University of Illinois at Urbana-Champaign, Champaign, IL, USA

Keywords Non-ability-based confidence, Overconfidence, Personality, Cognitive ability

Non-ability-based confidence is the discrepancy between one's confidence in their abilities and their actual ability. Some people are overconfident while others are underconfident. Here, we examined whether non-ability-based confidence is domain general or domain specific in middle and high school aged children. Are adolescents who are overconfident in one domain (e.g., math) also overconfident in another domain (e.g., German)? Using data from the German TwinLife project $(\mathrm{N}=5236$ individuals; $913 \mathrm{MZ}$ twin pairs, $2246 \mathrm{DZ}$ twin/full sibling pairs), we used a latent variable residual score approach to identify non-ability-based confidence for general ability, as well as for domain specific math and German ability. We found that general non-ability-based confidence was strongly correlated with math $(r=0.469)$ and German $(r=0.437)$ non-ability-based confidence, but that math and German non-ability-based confidence were weakly negatively related to each other $(r=-0.098)$. We decomposed the variance in each form of non-ability-based confidence and found that variance was attributable to genetic and nonshared environmental factors. Mirroring the phenotypic results, we found positive genetic correlations between general non-ability-based confidence and domain specific non-ability-based confidence and negligible genetic correlations between German and math non-ability-based confidence. Additionally, we found moderate non-shared environmental correlations suggesting that the twin higher on general nonability-based confidence also tended to be higher on math and German non-ability-based confidence. Taken together, these results 
suggest that domain specific non-ability-based confidence shares much of its variance with general non-ability-based confidence, but that the specific domains do not strongly relate to one another.

Grant Support: Daniel A. Briley was supported by Jacobs Foundation Research Fellowships. The German TwinLife Project is supported by the German Research Foundation (RI 595/8-1).

\section{Genetic Mechanisms of Intergenerational Transmission of Hyperactivity and Inattention}

Ivan Voronin ${ }^{1}$, Genevieve Morneau-Vaillancourt ${ }^{1}$,

Till F. M. Andlauer ${ }^{2}$, Stéphane Paquin ${ }^{1}$, Mara R. Brendgen ${ }^{3}$, Frank Vitaro ${ }^{4}$, Ginette Dionne ${ }^{1}$, Isabelle Ouellet-Morin ${ }^{5}$,

Michel Boivin ${ }^{1}$

${ }^{1}$ École de psychologie, Université Laval, Canada

${ }^{2}$ Department of Neurology, Technical University of Munich,

Germany

${ }^{3}$ Département de psychologie, Université du Québec à Montréal, Canada

${ }^{4}$ École de psychoéducation, Université de Montréal, Canada

${ }^{5}$ School of Criminology, University of Montreal, Montreal, Canada

Keywords ADHD, Hyperactivity, Inattention, Polygenic risk score, Educational attainment

The symptoms of ADHD, commonly classified into hyperactive and inattentive dimensions, are highly heritable and polygenic. The children of parents with ADHD tend to present more severe ADHD symptoms, and it is assumed that the intergenerational transmission of hyperactivity and inattention is genetically mediated. Recent research showed that parental genotype can predict a child's trait both directly through alleles passed on at conception, but also indirectly through parental behaviors (nature of nurture), independently of the genes transmitted to the children. The present study aims to document genetic mechanisms of intergenerational transmission of hyperactivity and inattention in a sample of twin families from Quebec, Canada. We used the data of 317 families from the Quebec Newborn Twin Study, 317 children and 394 parents in total. Hyperactivity and inattention of twins were repeatedly reported by parents in early childhood and by teachers in primary school. We derived polygenic risk scores (PRS) for ADHD and educational attainment to capture genetic variability associated with the ADHD symptoms. In preliminary analyses, twins' polygenic scores explained up to $6 \%$ of individual differences of inattention and up to $3 \%$ of hyperactivity. The parents' polygenic scores enhanced this prediction, with total explained variance up to $8 \%$ for inattention and $5 \%$ for hyperactivity. Overall, the PRS for ADHD was less predictive than the PRS for educational attainment: it explained at most $3.5 \%$ of the variance (early childhood hyperactivity). The results suggest that parental genotype can shape child's hyperactivity and inattention bypassing the child's own genotype, supposedly through environmental pathways.

Grant Support: Supported by the Quebec Network for Suicide, Mood Disorders, and Related Disorders (RQSHA) platform, partial stipend for graduate students, 2020-2021.

\section{Multi-polygenic Score Approach to Understanding the Intergenerational Transmission of Educational Achievement Across Childhood and Adolescence}

Biyao Wang ${ }^{1}$, Jessie R. Baldwin ${ }^{1,2}$, Tabea Schoeler ${ }^{1}$,

Rosa Cheesman $^{2,3}$, Wikus Barkhuizen ${ }^{1}$, Frank Dudbridge ${ }^{4}$,

David Bann ${ }^{5}$, Tim T. Morris ${ }^{6,7}$, Jean-Baptiste Pingault ${ }^{1,2}$

${ }^{1}$ Division of Psychology and Language Sciences, University College London, London, United Kingdom

${ }^{2}$ Social, Genetic and Developmental Psychiatry Centre, Institute of Psychiatry, King's College, London, United Kingdom

${ }^{3}$ PROMENTA Research Center, Department of Psychology, University of Oslo, Oslo, Norway

${ }^{4}$ Department of Health Sciences, University of Leicester, Leicester, United Kingdom

${ }^{5}$ Centre for Longitudinal Studies, Social Research Institute, University College London, London, United Kingdom

${ }^{6}$ Medical Research Council Integrative Epidemiology Unit, University of Bristol, Bristol, United Kingdom

${ }^{7}$ Population Health Sciences, Bristol Medical School, University of Bristol, Bristol, United Kingdom

Keywords ALSPAC, Educational achievement, Longitudinal development, Intergenerational transmission, Genetic nurture

Educational development in childhood and adolescence is associated with one's educational attainment, as well as major psychological, social, economic and health milestones throughout the life course. It is thus crucial to understand the processes underlying the intergenerational transmission of educational outcomes during these key developmental stages, which leads to continuing cycles of inequalities across generations. The intergenerational transmission of educational achievement is the mixture of genetic and environmental effects, including direct genetic effects originating in the offspring, and indirect genetic effects (genetic nurture) originating in the parents influencing offspring outcomes via environmental pathways. We used data from the Avon Longitudinal Study of Parents and Children (ALSPAC), a UK cohort study, and included over 1,300 motherfather-child trios. Scores of educational performances at four major Key Stages of education in the UK (age 7, 11, 14 and 16) were obtained through data linkage to the UK National Pupil Database to model the development course of educational achievements. Multiple polygenic scores were used, including educational attainment and its cognitive and noncognitive components, cognitive traits, psychiatric traits, personality, risk behaviors, reproductive behaviors, SES-related traits and health-related traits. There was strong evidence that genetic propensities of multiple traits were associated with cross-sectionally and the development of educational achievement, which can be attributed to both direct genetic effects and genetic nurture. These findings highlight the contribution of parental genetic influences to the intergenerational transmission of educational inequalities, which should justify for compensatory interventions.

Grant Support: B.W. and JB.P. are funded by a Nuffield Foundation project (EDO/43939); J.R.B is funded by a Wellcome Trust Sir Henry Wellcome fellowship (215917/Z/19/Z); T.S. is funded by a Wellcome Trust Sir Henry Wellcome fellowship (218641/Z/19/Z). D.B. is funded by the Economic and Social Research Council (ES/M001660/1) and Medical Research Council (MR/V002147/1). 


\section{Investigating a Potential Causal Relationship Between Maternal Blood Pressure During Pregnancy and Future Offspring Cardiometabolic Health}

Geng Wang ${ }^{1, \#}$, Laxmi Bhatta ${ }^{2, \#}$, Gunn-Helen Moen ${ }^{1,2,3,4}$, Liang-Dar Hwang ${ }^{1}$, John P Kemp ${ }^{1,5}$, Tom A. Bond ${ }^{1,4,5}$, Bjørn Olav Åsvold ${ }^{2,6}$, Ben Brumpton ${ }^{2,7,8, *}$, David M Evans ${ }^{1,5, *}$, Nicole M. Warrington ${ }^{1,2, *}$

${ }^{1}$ The University of Queensland Diamantina Institute, The University of Queensland, Brisbane, Australia

${ }^{2}$ K.G. Jebsen Center for Genetic Epidemiology, Department of Public Health and Nursing, NTNU, Norwegian University of Science and Technology, Trondheim, Norway

${ }^{3}$ Institute of Clinical Medicine, Faculty of Medicine, University of Oslo, Oslo, Norway

${ }^{4}$ Population Health Sciences, Bristol Medical School, University of Bristol, Bristol, UK

${ }^{5}$ Medical Research Council Integrative Epidemiology Unit, University of Bristol, Bristol, UK

${ }^{6}$ Department of Endocrinology, Clinic of Medicine, St. Olavs Hospital, Trondheim University Hospital, Trondheim, Norway

${ }^{7}$ Clinic of Medicine, St. Olavs Hospital, Trondheim University Hospital, Trondheim, Norway

${ }^{8}$ HUNT Research Center, Department of Public Health and Nursing, NTNU, Norwegian University of Science and Technology, Levanger, Norway.

${ }^{\#}$ These authors contributed equally to this work.

*These authors jointly supervised this work.

Keywords DOHaD, Cardiometabolic risk, Mendelian Randomization, Maternal blood pressure

Observational epidemiological studies have reported that higher maternal blood pressure during pregnancy is associated with increased future risk of offspring cardiometabolic disease. However, it is unclear whether this association represents a causal relationship through intrauterine mechanisms. We used a Mendelian randomization (MR) framework to examine the potential causal relationship between unweighted maternal genetic scores for systolic blood pressure (SBP) and diastolic blood pressure (DBP), and a range of cardiometabolic risk factors in the offspring of up to 29,708 genotyped mother-offspring pairs from the UK Biobank (UKB) and the Trøndelag Health (HUNT) studies. The cardiometabolic risk factors included in our analysis were systolic blood pressure, diastolic blood pressure, body mass index, lipid profile, glycemic biomarkers and other relevant traits. We conducted similar analyses in up to 21,423 father-offspring pairs from the same cohorts as a negative control for postnatal effects. We did not detect any association between maternal (or paternal) unweighted genetic scores for blood pressure and offspring cardiometabolic outcomes in the meta-analysis of UKB and HUNT analyses after adjusting for offspring genotypes at the same loci. We find little evidence to support the notion that normal variation in maternal blood pressure is a major causal risk factor for adverse offspring cardiometabolic outcomes in later life. These results complement the findings from conventional epidemiological studies, and indicate that offspring genetic effects or/and other environmental factors predominantly increase offspring cardiometabolic disease risk.

Grant Support: G.W. is supported by UQ Graduate School Scholarship (UQGSS). D.M.E. is funded by an Australian National Health and Medical Research Council Senior Research Fellowship (APP1137714) and this work was funded by NHMRC project grants (GNT1157714, GNT1183074). JPK is funded by a National Health and Medical Research Council (Australia) Investigator grant (GNT1177938). G.H.M. is supported by the Norwegian Research
Council (Post doctorial mobility research grant 287198), the Norwegian Diabetes Association and Nils Normans minnegave.T.A.B works in/is affiliated with a unit that is supported by the UK Medical Research Council (MC_UU_00011/6), and is supported by the British Heart Foundation Accelerator Award at the University of Bristol (R100643-101). L.B., B.Å.O. and B.M.B. receive support from the K.G. Jebsen Center for Genetic Epidemiology funded by Stiftelsen Kristian Gerhard Jebsen; Faculty of Medicine and Health Sciences, NTNU; The Liaison Committee for education, research and innovation in Central Norway; and the Joint Research Committee between St. Olavs Hospital and the Faculty of Medicine and Health Sciences, NTNU. The genotyping in HUNT was financed by the National Institute of Health (NIH); University of Michigan; The Research Council of Norway; The Liaison Committee for education, research and innovation in Central Norway; and the Joint Research Committee between St. Olavs Hospital and the Faculty of Medicine and Health Sciences, NTNU. The HUNT Study is a collaboration between the HUNT Research Centre (Faculty of Medicine and Health Sciences, NTNU, Norwegian University of Science and Technology), Trøndelag County Council, Central Norway Regional Health Authority, and the Norwegian Institute of Public Health.

\section{Familial Co-aggregation and Shared Heritability Between Depression, Anxiety, Obesity and Substance Use}

Rujia Wang ${ }^{1}$, Harold Snieder ${ }^{1}$, Catharina A. Hartman ${ }^{2}$

${ }^{1}$ Department of Epidemiology, University of Groningen, University Medical Center Groningen, Groningen, Netherlands

${ }^{2}$ Department of Psychiatry, University of Groningen, University Medical Center Groningen, Groningen, Netherlands

Keywords Familial co-aggregation, Depression, Anxiety, Obesity, Substance use

Depression, anxiety, obesity and substance use are heritable and often co-occur. However, the mechanisms underlying this co-occurrence are not fully understood. We estimated their familial aggregation and co-aggregation as well as heritabilities and genetic correlations to improve etiological understanding. Data came from the multi-generational population-based Lifelines Cohort Study ( $\mathrm{n}=162,439)$. Current depression and anxiety were determined using the MINI International Neuropsychiatric Interview. Smoking, alcohol and drug use were assessed by self-report questionnaires. Body mass index (BMI) and obesity were calculated by measured height and weight. Modified Cox proportional hazards models estimated recurrence risk ratios $\left(\lambda_{R}\right)$, and restricted maximum likelihood variance decomposition methods estimated heritabilities $\left(\mathrm{h}^{2}\right)$ and genetic correlations $\left(\mathrm{r}_{\mathrm{G}}\right)$. All analyses were adjusted for age, age ${ }^{2}$, and sex. Depression, anxiety, obesity and substance use aggregated within families $\left(\lambda_{\mathrm{R}}\right.$ firstdegree relative $=1.08-2.74)$ as well as between spouses $\left(\lambda_{R}=1.11-6.60\right)$. All phenotypes were moderately heritable (from $\mathrm{h}^{2}{ }_{\text {depression }}=0.25$ to $\mathrm{h}_{\text {BMI }}^{2}=0.53$ ). Depression, anxiety, obesity and smoking showed positive familial co-aggregation. That is, each of these traits confers increased risk on the other ones within families, consistent with the positive genetic correlations between these phenotypes $\left(\mathrm{r}_{\mathrm{G}}=\right.$ 0.16-0.94). The exception was obesity, which showed a "protective" co-aggregation pattern with alcohol and drug use and vice versa, consistent with the negative genetic correlations of BMI with alcohol $\left(r_{G}=-0.14\right)$ and drug use $\left(r_{G}=-0.05\right)$. Patterns of cross-phenotype recurrence risk highlight the co-occurrence among depression, anxiety, obesity and substance use within families. Patterns of genetic 
overlap between these phenotypes provide clues to uncovering the mechanisms underlying familial co-aggregation.

Grant Support: The Lifelines Cohort Study is supported by the Netherlands Organization of Scientific Research NWO (grant 175.010.2007.006), the Economic Structure Enhancing Fund (FES) of the Dutch government, the Dutch Ministry of Economic Affairs, the Ministry of Education, Culture and Science, the Dutch Ministry of Health, Welfare and Sports, the Northern Netherlands Collaboration of Provinces (SNN), the Province of Groningen, the University Medical Center Groningen, the University of Groningen, Dutch Kidney Foundation and Dutch Diabetes Research Foundation. Rujia Wang was supported by the China Scholarship Council (201806010404).

\section{Genetic Correlates and Consequences of Phenotypic Heterogeneity in Autism}

Varun Warrier ${ }^{1}$, Yira Zhang ${ }^{1}$, Freddy Cliquet $^{2}$, Jakob Grove ${ }^{3}$, Hilary Martin $^{4}$, Thomas Rolland ${ }^{2}$, Celia van der Merwe ${ }^{5}$, Claire LeblondManry $^{2}$, Anders Borglum ${ }^{3}$, Elise Robinson ${ }^{5}$, Thomas Bourgeron ${ }^{2}$, Simon Baron-Cohen ${ }^{1}$

${ }^{1}$ Department of Psychiatry, University of Cambridge, Cambridge, UK ${ }^{2}$ Institut Pasteur, Paris, France

${ }^{3}$ Department of Biomedicine, Aarhus University, Aarhus, Denmark

${ }^{4}$ Wellcome Trust Sanger Institute, Hinxton, UK

${ }^{5}$ Harvard T.H. Chan School of Public Health, Harvard, Boston, Massachusetts, USA

\section{Keywords Autism, Heterogeneity, Sex differences}

The substantial heterogeneity in autism is a challenge for investigating the aetiology of autism and translating research findings to support autistic people. To address this, we first conducted factor analyses of the autistic traits questionnaire 24,000 autistic individuals and identified six correlated factors: 1 . Insistence on sameness; 2. Social Interaction; 3. Sensory-motor; 4. Self-harm; 5. Idiosyncratic communication; 6. Conversational skills. Factor analyses improved the interpretability of results and SNP heritability of the autism features compared to summed scores. Using data from 19 different core and associated autism features, we identified significant associations between multiple genetic risk factors and autism features, including with autism PGS. High impact de novo variants and autism PGS were independently associated with autism liability and are associated with different features. For instance, PGS for educational attainment and autism were associated with several of the six factors. In contrast, high impact de novo variants were associated primarily with associated autism features. We investigated differences in PGS based on ID and sex. Notably, we identified a significant over-transmission of autism PGS in females without ID compared to males. This effect increased when restricting to individuals with average or above average IQ. There were modest differences in SNP heritabilities between different definitions of autism. Finally, GWAS of autism features in autistic individuals had modest genetic correlations with autism. Heterogeneity in autism is complex and multifactorial and impacts the interpretability of genetic findings in autism.

Grant Support: Wellcome Trust
Do Adult ADHD Symptoms Share Genetic Underpinnings with Child ADHD Symptoms and Those of Related Comorbid Childhood Disorders? Comparing Within-Child and Mother-Child Genetic Correlations in a Large Norwegian Children-of-Twins-and-Siblings Cohort

Daniel L Wechsler ${ }^{1}$, Fruhling V Rijsdijk ${ }^{1}$, Espen M Eilertsen ${ }^{2,3}$, Eivind Ystrom ${ }^{2,3,4}$, Yasmin I Ahmadzadeh ${ }^{1}$, Isabella Badini ${ }^{1}$, Laurie J Hannigan $^{5,6}$, Tom A McAdams ${ }^{1,2}$

${ }^{1}$ Social, Genetic and Developmental Psychiatry Centre, Institute of Psychiatry, Psychology and Neuroscience, King's College London, London, United Kingdom

${ }^{2}$ PROMENTA Research Centre, University of Oslo, Oslo, Norway ${ }^{3}$ Department of Mental Disorders, Norwegian Institute of Public Health, Oslo, Norway

${ }^{4}$ School of Pharmacy, University of Oslo, Oslo, Norway

${ }^{5} \mathrm{Nic}$ Waals Institute, Lovisenberg Diaconal Hospital, Oslo, Norway

${ }^{6}$ Medical Research Council Integrative Epidemiology Unit, University of Bristol, Bristol, UK

Keywords ADHD, Comorbidity, Genetics, Mother, Child

Addressing the debate about whether ADHD symptomatology in childhood and adulthood could be etiologically distinct, we used a novel quantitative genetic approach in a large family dataset to assess whether ADHD symptoms in mothers and children shared similar genetic correlations with symptoms of ADHD-related comorbid disorders in children. Genetic correlations were derived from two methodologies: (1) Extended bivariate twin analyses (including siblings and cousins) assessing within-child genetic overlap; (2) Multiple-Children-of-Twins-and-Siblings analyses assessing between-mother-and-child genetic overlap. Both sets of analyses used a common sample taken from the Mother and Child Cohort Study (MoBa), a large Norwegian birth registry cohort of $\sim 115,000$ pregnancies. Maternal ADHD symptoms were assessed at child age 3 years, and child ADHD symptoms were assessed at age 5 years. Comorbid symptom measures were child ODD, conduct disorder, anxiety and depression symptoms, all at age 8 years. High genetic correlations were found between maternal ADHD symptoms and child ADHD and comorbid symptoms. Shared genetic influences accounted for the majority of all mother-child phenotypic associations, closely reflecting the high proportions of within-child phenotypic associations explained by genetic overlap. Using a novel family-based approach, our results provide evidence that ADHD symptoms in children, and in their adult parents, share comparable genetic overlap with children's later symptoms of several common ADHD-related disorders. This suggests that symptoms of ADHD as measured in the adult mothers in our sample are not etiologically distinct to those measured in children, as they are related to a wider array of ADHD-related comorbid symptomatology in their children.

Grant Support: DLW is supported by the UK Medical Research Council (MR/N013700/1) and King's College London member of the MRC Doctoral Training Partnership in Biomedical Sciences. TAM and YIA are supported by a Wellcome Trust Senior Research Fellowship awarded to TAM (220382/Z/20/Z). EY (288083; 262177) and TAM (288083) are supported by the Research Council of Norway. EME is supported by the Norwegian Research Council (262177 and 288083). IB is supported by the UK Economic and Social Research Council (ESRC) and King's College London member of the ESRC Doctoral Training Partnership in Interdisciplinary Social Science (ST11872). 


\section{The Predictive Value and Potential Pathways of a Polygenic Score for Rhythm Ability}

\author{
Laura W. Wesseldijk $k^{1,2}$, Fredrik Ullén ${ }^{1,3}$, Miriam A. Mosing ${ }^{1,4,5}$ \\ ${ }^{1}$ Department of Neuroscience, Karolinska Institutet \\ ${ }^{2}$ Department of Psychiatry, Amsterdam UMC, University of \\ Amsterdam \\ ${ }^{3}$ Department of Cognitive Neuropsychology, Max Planck Institute for \\ Empirical Aesthetics, Frankfurt \\ ${ }^{4}$ Department of Medical Epidemiology and Biostatistics, Karolinska \\ Institutet \\ ${ }^{5}$ Melbourne School of Psychological Sciences, Faculty of Medicine, \\ Dentistry, and Health Sciences, University of Melbourne
}

Keywords Music skills, Polygenic score, Genetics, Family data, Geneenvironment correlation

Genetics play a role in the etiology of music skills; however, still little is understood about the genetic architecture of music-related skills and traits. To further our understanding of the genetic underpinnings of music skills, we explored associations between a polygenic score for self-reported rhythm ability $\left(\mathrm{PGS}_{\text {rhythm }}\right)$ and objectively measured rhythm ability, as well as other validated music-related skills and traits. Utilizing family data, we could further explore potential pathways of direct genetic, indirect genetic (through passive geneenvironment correlation) and confounding effects (such as assortative mating). In a genetically informative sample of 5,648 Swedish twins, we found the PGS $_{\text {rhythm }}$ to predict not only rhythm ability, but also other music skills (betas ranging between .12 and .14, $\mathrm{p}<.001$ ), as well as music-related outcomes (betas between -.03 and $.10, \mathrm{p}<.05$ ). Associations did not deteriorate within families, implying that indirect genetic or confounding effects did not inflate the effect of the PGS $_{\text {rhythm. In contrast, }}$ PGS rhythm was not associated with any of the investigated control phenotypes not immediately related to music skills, such as general intelligence and sport practice. A small but significant correlation $(\mathrm{r}=.05, \mathrm{p}<.001)$ between the level of musical enrichment of the family childhood environment and individuals' $\mathrm{PGS}_{\text {rhythm, }}$ provides evidence for gene-environment correlation for music-related traits. Furthermore, the PGS $_{\text {rhythm }}$ correlation between dizygotic twin members $(\mathrm{r}=.54, \mathrm{CI} .50-.58)$ was somewhat higher than expected, suggesting assortative mating. We conclude that the $\mathrm{PGS}_{\text {rhythm }}$ reflects individuals' more general musicality propensity, affecting music behavior essentially through direct genetic effects.

Grant Support: This work was supported by the Bank of Sweden Tercentenary Foundation (M11-0451:1), the Sven and Dagmar Salén Foundation, and the Marcus and Amalia Wallenberg Foundation (MAW 2018.0017). The Swedish Twin Registry is managed by the Karolinska Institutet and receives funding through the Swedish Research Council (2017-00641).

\section{Genomic Prediction of Education Outcomes: A Systematic Review}

Kirsty Wilding ${ }^{1}$, Allie Nancarrow ${ }^{1}$, Sophie von Stumm ${ }^{1}$ ${ }^{1}$ Department of Education, University of York, York, UK

Keywords Polygenic scores, Education, Systematic review

Genome-wide polygenic scores (GPS) aggregate DNA variants identified in genome-wide association (GWA) studies to index an individual's genetic propensity for a trait. Here, we present a systematic review of studies that tested associations between GPS, derived from the summary statistics of the largest GWA study for education attainment (Lee et al., 2018, Nat Genet) and phenotypic measures of education outcomes (preregistered: OSF https://osf.io/ xcm43/; PROPSERO https://www.crd.york.ac.uk/prospero/displ ay_record.php?ID=CRD42021237620). To address (a) the strength of association between GPS and education outcomes across studies and (b) the role of moderators on the association (e.g., type of education outcome, participant characteristics, and GPS computation methods), Google Scholar and Web of Science searched for papers that cited Lee et al.'s GWA. 1100 hits were returned; after removing duplicates and excluding ineligible papers, 36 studies met the eligibility criteria, reporting an association between GPS and a phenotypic measure of education in independent populations. GPS predicted education with small to medium effect sizes $\left(\mathrm{R}^{2}\right.$ from .04 to .16) for a range of outcomes, from school performance to highest education qualification level obtained. The age of phenotypic education assessment ranged from 7 to 50, and differences in strength of association varied minimally by age at or type of phenotypic assessment, or by population origin, or GPS estimation methods. Of these studies, 93\% tested exclusively samples of European Ancestry. Our review summarizes that individuals with higher genetic propensities for education attainment perform better throughout compulsory schooling and are more likely to continue onto further education (e.g., university).

Grant Support: Nuffield Foundation grant awarded to S.v.S (EDO/ 44110)

\section{Genetic and Environmental Influences on Alzheimer's Disease Neuroimaging Signatures}

McKenna E. Williams ${ }^{1,2,3}$, Nathan A. Gillespie ${ }^{4}$, Tyler R. Bell ${ }^{1,3}$, Anders M. Dale ${ }^{5,6}$, Jeremy A. Elman ${ }^{1,3}$, Lisa T. Eyler, ${ }^{3,7}$,

Christine Fennema-Notestine ${ }^{3,5}$, Donald J. Hagler, Jr. ${ }^{5}$,

Linda K. McEvoy ${ }^{5}$, Michael C. Neale ${ }^{4}$, Matthew S. Panizzon ${ }^{1,3}$, Chandra A. Reynolds ${ }^{8}$, Mark Sanderson-Cimino ${ }^{1,2,3}$,

Carol E. Franz ${ }^{1,3}$, William S. Kremen, $\mathrm{PhD}^{1,3}$

${ }^{1}$ Center for Behavior Genetics of Aging, University of California San Diego, La Jolla, CA, USA

${ }^{2}$ Joint Doctoral Program in Clinical Psychology, San Diego State University/University of California, San Diego, CA, USA

${ }^{3}$ Department of Psychiatry, University of California San Diego, La Jolla, CA, USA

${ }^{4}$ Virginia Institute for Psychiatric and Behavior Genetics, Virginia Commonwealth University, VA, USA

${ }^{5}$ Department of Radiology, University of California San Diego, La Jolla, CA, USA

${ }^{6}$ Department of Neuroscience, University of California San Diego, La Jolla, CA, USA

${ }^{7}$ Desert Pacific Mental Illness Research Education and Clinical Center, VA San Diego Healthcare System, CA, USA

${ }^{8}$ Department of Psychology, University of California Riverside, Riverside, CA, USA

Keywords Alzheimer's disease, Mean diffusivity, Cortical thickness, Brain age

Composite scores of MRI-based morphometrics in brain regions associated with Alzheimer's disease (AD) pathology have been developed to distinguish early AD-related atrophy from normal ageassociated brain changes. These composite scores, commonly termed 'AD signatures,' are associated with increased risk of progression to mild cognitive impairment or AD and with symptom severity. Genetic and environmental influences on $\mathrm{AD}$ signatures have not been 
examined. Moreover, given that aging is associated with atrophy in areas that overlap with regions in $\mathrm{AD}$ signatures, it is unknown if they capture unique AD-related variance or simply reflect general brain aging. Here, we used a cortical thickness/hippocampal volume signature (CTHV), a mean diffusivity (MD) signature, and an MRIbased measure of predicted brain age $(n s=310-502)$ in biometrical analyses to determine (1) the genetic and environmental etiology of each AD signature and the measure of brain age, and (2) sources of covariance among these three phenotypes. Participants were from the Vietnam Era Twin Study of Aging (age $=56.18$, SD = 2.61). Heritability estimates were similar for the signatures and predicted brain age (0.68-0.71). Phenotypic correlations ranged from -0.46 to 0.27 . Genetic correlations among the three phenotypes were moderate but significant: 0.33 [95\% CI 0.19,0.47] (CTHV signature and predicted brain age); -0.55 [95\% CI $-0.66,-0.42]$ (MD signature and predicted brain age); and -0.36 [95\% CI $-0.51,-0.20]$ (CTHV and MD signatures). Despite similar heritability estimates and significant genetic correlations among the three phenotypes, our findings are consistent with genetic influences unique to each $\mathrm{AD}$ signature that were not shared with those contributing to general brain aging.

Grant Support: This work was assisted through funding from the National Institute on Aging [R01 AG022381, R01 AG050595].

\section{Parent Contributions to the Development of Political Attitudes in Adoptive and Biological Families}

Emily A. Willoughby ${ }^{1}$, Alexandros Giannelis ${ }^{1}$, Steven Ludeke $^{2}$, Robert Klemmensen ${ }^{3}$, Asbjørn S. Nørgaard ${ }^{4}$, William G. Iacono ${ }^{1}$, James J. Lee ${ }^{1}$, and Matt McGue ${ }^{1}$

${ }^{1}$ Department of Psychology, University of Minnesota Twin Cities, Minneapolis, Minnesota, USA

${ }^{2}$ Department of Psychology, University of Southern Denmark, Odense, Denmark

${ }^{3}$ Department of Political Science, University of Southern Denmark, Odense, Denmark

${ }^{4}$ CEVEA, Copenhagen, Denmark

Keywords Political attitudes, Adoption, Behavior genetics, Environment

Where do our political attitudes originate? While early research attributed the formation of such beliefs to parent and peer socialization, genetically sensitive designs later clarified the substantial role of genes in the development of sociopolitical attitudes. However, it has remained unclear whether parental influence on offspring attitudes persists beyond adolescence. In a unique sample of 394 adoptive and biological families with offspring over the age of 30 , biometric modeling revealed significant evidence for genetic and non-genetic transmission from both parents for the majority of seven political attitude phenotypes. We found the largest genetic effects for religiousness and social liberalism, while the largest influence of parental environment is seen for political orientation and egalitarianism. Together, these findings indicate that genes, environment, and geneenvironment correlation all contribute significantly to sociopolitical attitudes held in adulthood, the etiology and development of which may be more important than ever in today's rapidly changing sociopolitical landscape.

Grant Support: This research was funded by a grant from the John Templeton Foundation as part of their Genetics and Human Agency initiative (Grant Number 60780), and original assessment data collection was funded by the National Institutes of Health (Grant Numbers MH066140 and AA011886).

\section{Genetic Identification of Sleep Vulnerabilities Inherited with Substance Use}

Evan A. Winiger ${ }^{1}$, Alexander S. Hatoum ${ }^{2}$ C. Lucia Morrison ${ }^{1}$, Emma C. Johnson ${ }^{2}$, \& Arpana Agrawal ${ }^{2}$

${ }^{1}$ Institute for Behavioral Genetics, University of Colorado Boulder

${ }^{2}$ Washington University School of Medicine, Department of Psychiatry

Keywords Sleep, Substance Use, Activity

The overlap between substance use and sleep deficits is one of the most common clinical concerns in treatment. Substance use may affect sleep in general or manifest as specific sleep deficits. Here, we test the genetic overlap between substance use behaviors and sleep/ activity measures, derive genetic clusters between these domains, and test processes of causality vs. pleiotropy, in the largest public samples of substance use behaviors and sleep/activity phenotypes/endophenotypes to date. We found 31 genetic correlations between substance use and sleep/activity measures that survived Bonferroni correction. Several of the discovered genetic correlations include novel relationships between substance use behaviors and objective measures of sleep/activity, while numerous of the genetic correlations confirm past work on substance use behaviors and subjective sleep measures. We found two broad genetic clusters that underly a pattern of coinheritance that characterizes the overlap of substance use and sleep. Genes associated with tobacco use severity shared overlap with elements of sleep health (sleep duration, sleep efficiency, and chronotype). Substance consumption and problematic substance use behaviors (use disorders and problematic use) clustered strongly with problematic measures of sleep as well as measures of activity. Latent causal variable analyses determined that pleiotropy likely underlies a majority of the associations between substance use and sleep/activity measures, with the caveat of one significant result which implied genetic causality for opiate use disorder on self-reported long sleep duration. Overall, these combined results imply a strong shared genetic relationship between the domains of substance use behaviors and sleep/activity measures.

Grant Support: EAW receives support from HD007289-30. ASH receives support from DA007261-17. CLM receives support from MH016880. ECJ receives support from AA027435-02. AA receives support from MH109532 and K02DA032573. Conflict of interest disclosures: No disclosures were reported.

\section{The Heritability of Educational Attainment - Shared Environment, Genetic Nurture and Psychological Mediators}

Tobias Wolfram

${ }^{1}$ Department of Sociology, University of Bielefeld, Bielefeld, Niedersachen, Germany

Keywords Nuclear twin family design, Genetic nurture, Educational attainment

Variation in educational attainment is caused by both genetic and environmental factors. Environmental influences might either be the 
result of resources shared between children in the same family or due to unsystematic, nonshared factors. We leverage a nuclear twin family design and analyze educational attainment of German twins, their siblings and parents to disentangle different mechanisms of the shared environment. In line with past findings, genetic effects explain half, the shared environment a quarter of the observed variation. Effects strongly differ for different sources of the shared environment: In contrast to recent molecular-genetic findings concerning genetic nurture, vertical transmission from parent to offspring is weak and nonsignificant. Instead, proximal (shared between twins) and distal (shared between all siblings) factors dominate. By controlling for measured psychological covariates we show that both proximal and distal effects affect educational attainment by influencing (among other things) noncognitive traits, indicating socially mediated skilltransfer in addition to genetic pathways.

\section{Dynamic Associations Between Physical and Cognitive Development in Twins Across Early Childhood}

Sean R. Womack ${ }^{1}$, Evan J. Giangrande ${ }^{1}$, Ramona Weber $^{1}$, Christopher R. Beam ${ }^{2}$, Deborah W. Davis ${ }^{3}$, Eric Turkheimer ${ }^{1}$

${ }^{1}$ Department of Psychology, University of Virginia, Charlottesville, Virginia, USA

${ }^{2}$ Department of Psychology, University of Southern California, Los Angeles, California, USA

${ }^{3}$ Department of Pediatrics, University of Louisville, Louisville, Kentucky, USA

Keywords Cognitive development, Height, Weight, Louisville Twin Study

Owing to high rates of premature birth, twins are often born at low birth weight and display depressed cognitive functioning in infancy and toddlerhood. However, by early childhood, twins typically have "recovered" to the population mean of physical growth (e.g., height and weight) and cognitive ability. Cognitive recovery follows an S-shaped curve characterized by minimal gain across infancy, rapid recovery across toddlerhood, and stabilization around the population mean by early childhood. In contrast, recovery in height and weight begins in early infancy and stabilizes around the population mean by early toddlerhood. Thus, developmentally, twins appear to recovery physically before recovering cognitively. However, temporal associations between physical and cognitive recovery have not been tested in twins. Using longitudinal data from the Louisville Twin Study, we examine temporal associations between physical growth and standardized cognitive ability scores from infancy to middle-childhood using age appropriate measures (Bayley, Stanford-Binet, WPPSI, WISC). Specifically, we examine the dynamic association between physical growth and cognitive ability using a latent change score framework. Models were fit separately for height and weight. Results were consistent across height and weight models and suggest that physical growth anticipates cognitive growth. Findings provide insight into the developmental process by which twins recover physically and cognitively across childhood, and may provide a model for understanding how both twins and singleton children recover from early bio-environmental adversity (e.g., premature birth, anoxia, at low birth weight).

Grant Support: This work was supported by the National Institute on Aging (R03AG048850-01 and R01AG063949) and the John Templeton Foundation's Genetics and Human Agency initiative.
Genetic and Environmental Etiology of Political Sophistication in Japan: A Study of Adolescent Twins and Their Parents

Shinji Yamagata ${ }^{1}$

${ }^{1}$ Department of Psychology and Human Developmental Sciences, Nagoya University, Aichi, Japan

Keywords Self-direction, Value, Adolescence, Twin study

This study examined genetic and environmental etiology of Japanese adolescents' political sophistication by utilizing an extended twinfamily design. A data of 751 twins, 402 mothers, and 318 fathers from 424 families were collected by a cross-sectional survey in March 2010. Univariate analyses revealed that individual differences in political knowledge and interest were explained by shared and nonshared environmental influences $\left(c^{2}=.49\right.$ and .31 , respectively). Multivariate genetic analyses revealed that political interest, parental political knowledge, and political conversation with parents together explained $43 \%$ of shared environmental variance of adolescents' political knowledge, where fathers than mothers played a greater role in the effects of both knowledge and conversation. These results suggested that familial resemblance of adolescent's political sophistication in Japan was due not to genetic but to cultural transmission of parental political sophistication.

Grant Support: 07J05497 (Japan Society for the Promotion of Science).

\section{Genetic and Environmental Contributions to Physical, Verbal, and Relational Bullying Victimization in Middle Childhood}

\author{
Li Yu ${ }^{1}$, Valerie Knopik ${ }^{1}$, Kristine Marceau ${ }^{1}$, Laura Baker ${ }^{2}$ \\ ${ }^{1}$ Department of Human Development and Family Studies, Purdue \\ University, West Lafayette, Indiana, USA \\ ${ }^{2}$ Department of Psychology, University of Southern California, Los \\ Angeles, California, USA
}

Keywords Bullying, Behavior genetics, Childhood, Heritability

Bullying includes different forms (i.e., physical, verbal, relational), but genetically informed studies have rarely explored them separately. The only study to elucidate the contributions of genetic and environmental factors for different forms of bullying used teacher report (Veldkamp et al., 2019). The present study is the first to explore additive genetic and environmental contributions to separate forms of self-reported bullying victimization. In the USC longitudinal study of Risk Factors for Antisocial Behavior, 9-10-year-old twins (548 pairs) reported bullying victimization. $74 \%$ of the children reported being verbally bullied, whereas $35 \%$ reported being physically bullied, and $62 \%$ reported relational bullying victimization. Intraclass correlations (ICCs) of verbal bullying victimization suggested genetic and nonshared environmental influences: monozygotic $(\mathrm{MZ})_{\text {male }}=0.48$, $\mathrm{MZ}_{\text {female }}=0.43$, dizygotic $(\mathrm{DZ})_{\text {male }}=0.33, \mathrm{DZ}_{\text {female }}=0.20$, opposite sex dizygotic $(\mathrm{OZ})=0.36$. ICCs of physical bullying victimization indicated genetic or shared environmental and nonshared environmental influence: $\mathrm{MZ}_{\text {male }}=0.33, \mathrm{MZ}_{\text {female }}=0.33, \mathrm{DZ}_{\text {male }}=0.28$, $\mathrm{DZ}_{\mathrm{female}}=0.07 ; \mathrm{OZ}=0.28$. ICCs of relational bullying victimization indicated shared and nonshared environmental influence: $\mathrm{MZ}_{\text {male }}=$ $0.29, \mathrm{MZ}_{\text {female }}=0.23, \mathrm{DZ}_{\text {male }}=0.25, \mathrm{DZ}_{\text {female }}=0.26 \mathrm{OZ}=0.33$. The best-fitting univariate decompositions confirmed these predictions: 
verbal $\left(\mathrm{a}^{2}=49 \%, \mathrm{e}^{2}=51 \%\right)$, physical $\left(\mathrm{a}^{2}=36 \%, \mathrm{e}^{2}=64 \%\right.$, although $\mathrm{A}$ and $\mathrm{C}$ could each be dropped independently, indicating ambiguous familial influences), relational bullying victimization: $\left(c^{2}=24 \%, \mathrm{e}^{2}=\right.$ $76 \%$ ). Given sex differences in ICCs, we will also test sex limitation models.

\section{References}

Veldkamp, S. A., Boomsma, D. I., de Zeeuw, E. L., van Beijsterveldt, C. E., Bartels, M., Dolan, C. V., \& van Bergen, E. (2019). Genetic and environmental influences on different forms of bullying perpetration, bullying victimization, and their co-occurrence. Behavior genetics, 49(5), 432-443.

Grant Support: Dr. Baker's work was supported by NIMH \#MH58354. Dr. Marceau's time was supported by K01 DA039288.

\section{The Etiology of Nutrition and Cardio-Metabololic Phenotypes in a South Asian Population}

Helena M.S. Zavos ${ }^{1}$, Laura Riddleston ${ }^{1}$,

Kaushayla Jayaweera ${ }^{2}$, Gayani Pannala ${ }^{2}$, Sisira Siribaddana ${ }^{3}$, Matthew Hotopf $^{4}$, Athula Sumathipala ${ }^{2}$, Frühling V Rijsdijk ${ }^{5}$

${ }^{1}$ Department of Psychology, Institute of Psychiatry, Psychology \& Neuroscience, King's College London, UK

${ }^{2}$ Institute for Research and Development, Colombo, Sri Lanka

${ }^{3}$ Department of Medicine, University of Rajarata, Sri Lanka

${ }^{4}$ Psychological Medicine Department, Institute of Psychiatry, Psychology, and Neuroscience, King's College London, UK; NIHR Biomedical Research Centre for Mental Health at the South London and Maudsley NHS Foundation Trust, King's College London, UK ${ }^{5}$ Social Genetic and Developmental Research Centre, Institute of Psychiatry, Psychology \& Neuroscience, King's College London, UK

Keywords Nutrition, Cardio-metabololic phenotypes, South Asia, Diabetes, Low- and Middle-income countries (LMICs)

Low- and middle-income countries (LMICs) globally have undergone rapid urbanisation, and changes in demography and health behaviours. In Sri Lanka, cardio-vascular disease and diabetes are now leading causes of mortality. High prevalence estimates of their risk factors, including hypertension, dysglycaemia and obesity have also been observed. Nutrition and diet are key modifiable risk factors for both cardio-vascular disease and diabetes as well as their risk factors. Although typically thought of as environmental risk factors, dietary choice and nutrition have been shown to be genetically influenced and that genes associated with these behaviours correlate with metabolic risk indicators. We used Structural Equation Model fitting to investigate the etiology of nutrition and cardio-metabolic phenotypes in COTASS, a population-based twin and singleton sample in Colombo, Sri Lanka. Participants completed the Food Frequency Questionnaire $(\mathrm{N}=3934)$ which assessed frequency of intake of 14 food groups including 'meat', 'vegetables' and 'desert/sweet snacks'. Anthropometic $(\mathrm{N}=3675)$ and cardio-metabolic $(\mathrm{N}=3477)$ data was also collected including blood pressure, weight, cholesterol, fasting plasma glucose and triglycerides. Frequency of food intake was largely environmental, both shared and non-shared, in origin. Modest genetic influences were observed for certain food groups, for example 'meat' and 'fruit'. Cardio-metabolic phenotypes showed moderate genetic influences. Shared environmental influences were also observed for a number of phenotypes including BMI, blood pressure and triglycerides. Shared environmental effects were shown to be more important for both nutrition and cardio-metabolic phenotypes than previous research in Western high income populations. This emphasizes the importance of research conducted in diverse populations.

Grant Support: The COTASS study has been supported by the Wellcome Trust $(093206 / \mathrm{Z} / 10 / \mathrm{Z})$ and is currently supported by the Medical Research Council (MR/T00679X/1)

\section{Impacts of Recreational Cannabis Legalization on Cannabis Use and Other Drug Outcomes: A Longitudinal Discordant Twin Study}

Stephanie M. Zellers ${ }^{1}$, J. Megan Ross ${ }^{2}$, Jarrod M. Ellingson ${ }^{2,3}$, Robin P. Corley ${ }^{3}$, John K. Hewitt ${ }^{3,4}$, Christian J. Hopfer ${ }^{2,3}$, William Iacono ${ }^{1}$, Matt K. McGue ${ }^{1}$, and Scott I. Vrieze ${ }^{1}$

${ }^{1}$ Department of Psychology, University of Minnesota, Minneapolis, Minnesota, USA

${ }^{2}$ Department of Psychiatry, University of Colorado Anschutz Medical Campus, Aurora, Colorado, USA

${ }^{3}$ Institute for Behavioral Genetics, University of Colorado Boulder, Boulder, Colorado, USA

${ }^{4}$ Department of Psychology and Neuroscience, University of Colorado Boulder, Boulder, Colorado, USA

Keywords Cannabis legalization, Discordant twin, Cannabis frequency, Longitudinal

Seventeen states and Washington D.C. have legalized recreational cannabis use ("legalization"), despite limited understanding of its consequences. As laws change rapidly and dramatically, it is necessary to research the impacts of legalization on rates of use, disordered use, and psychosocial outcomes. Adult residents in such states report higher use after legalization but this work is largely cross-sectional and ignores relevant confounding factors, including cultural and secular trends in states that legalize versus those that do not. Longitudinal work using improved study designs is required to accurately estimate the environmental effect of the legalization event on individuals living in that state. We leveraged two adult twin studies, one from Colorado (legal cannabis) and one from Minnesota (recreational cannabis still illegal), both with harmonized longitudinal assessments before and after the legalization events (total $\mathrm{N}=3420$ ). We combined a discontinuity design with a discordant twin analysis to produce robust estimates of the environmentally mediated effect of legalization, largely free of confounding from other trends in cannabis use and attitudes. Residents of legal states use cannabis at higher rates than non-legal states (unstandardized $\mathrm{B}=.21, \mathrm{p}=7.7 \times 10^{-5}$ ); this effect corresponds to a $\sim 24 \%$ increase in cannabis use frequency attributable to legalization and it is robust to the inclusion of earlier use. Discordant twin analyses found no evidence of genetic or shared environmental confounding ( $\mathrm{MZ}$ unstandardized $\mathrm{B}=.18, \mathrm{p}=.01$ ), supporting a causal pathway between legalization and increased use. We also plan to present yet-unfinished work on other substance use outcomes.

Grant Support: This work was supported by grants from the National Institute of Drug Abuse (DA042755 CJH/JKH/MKM/SIV). 


\section{Detection of Assortative Mating in the Absence of Spousal Information}

Yuanxiang Zhang ${ }^{1}$, Allan F McRae ${ }^{1}$, Peter M Visscher ${ }^{1}$, Loic Yengo ${ }^{1}$ ${ }^{1}$ Institute for Molecular Bioscience, The University of Queensland, Brisbane, Australia

Keywords Assortative mating, GREML

Assortative mating, i.e., mate choice based on phenotypic similarity, increases the prevalence of extreme phenotypes the population. To date, only a few methods can detect AM in the absence of spousal information, which is scarce. Here, we introduce the between-chromosome Genome-based Maximum Likelihood (GREML-BC), a new method, which can detect AM by quantifying how much crosschromosomal genetic relatedness contributes to phenotypic resemblance in the population. In simulations, GREML-BC is statistically well powered to distinguish AM from random mating and statistical power increases monotonically with sample size and the degree of phenotypic resemblance between mates. We applied GREML-BC to 52 phenotypes measured on 347,819 unrelated European ancestry participants of the UK Biobank. We found significant evidence of AM for 15 complex traits, including fluid intelligence, smoking status, overall health rating, average household income and time spent watching TV. We show that AM on these traits is correlated with that on educational attainment, previously reported. In conclusion, GREML-BC is able to detect signal of AM using only genotypes and phenotypes of unrelated individuals, which makes it applicable to large biobank data where spousal information is absent.

Grant Support: This research was supported by the Australian Research Council (Grant number: DE200100425).

\section{Novel Disease Associations with Schizophrenia Genetic Risk Revealed in $\sim 400,000$ UK Biobank Participants}

Ruyue Zhang ${ }^{1}$, MSc, Arvid Sjölander ${ }^{1}, \mathrm{PhD}$, Alexander Ploner ${ }^{1}, \mathrm{PhD}$, Donghao $\mathrm{Lu}^{1,2}$, MD, PhD, Cynthia M. Bulik ${ }^{1,3,4}, \mathrm{PhD}$, Sarah E. Bergen $^{1}, \mathrm{PhD}$

${ }^{1}$ Department of Medical Epidemiology and Biostatistics, Karolinska Institutet, Stockholm, Sweden

${ }^{2}$ Department of Epidemiology, Harvard T.H. Chan School of Public Health, Boston, MA, USA

${ }^{3}$ Department of Psychiatry, University of North Carolina at Chapel Hill, Chapel Hill, USA

${ }^{4}$ Department of Nutrition, University of North Carolina at Chapel Hill, Chapel Hill, USA

Keywords Schizophrenia, Polygenic risk score, Pleiotropy, PheWAS

Schizophrenia is a serious mental disorder with considerable somatic and psychiatric morbidity. It is unclear whether comorbid health conditions predominantly arise due to shared genetic risk or consequent to having schizophrenia. To explore the contribution of genetic risk for schizophrenia, we analysed the effect of schizophrenia polygenic risk scores (PRS) on a broad range of health problems in 406,929 individuals with no schizophrenia diagnosis from UK Biobank. Diagnoses were derived from linked health data including primary care, hospital inpatient records, and registers with information on cancer and deaths. Schizophrenia PRS were generated and tested for associations with general health conditions, 16 ICD10 main chapters, and 603 diseases using linear and logistic regressions. Higher schizophrenia PRS was significantly associated with poorer overall health ratings, more hospital inpatient diagnoses, and more unique illnesses. It was also significantly positively associated with 4 ICD10 chapters: mental disorders; respiratory disease; digestive disease; and pregnancy, childbirth and the puerperium, but negatively associated with musculoskeletal disorders. Thirty-one specific phenotypes were significantly associated with schizophrenia PRS, and the 19 novel findings include several musculoskeletal diseases, respiratory diseases, digestive diseases, varicose veins, pituitary hyperfunction, and other peripheral nerve disorders. These findings extend knowledge of the pleiotropic effect of genetic risk for schizophrenia and offer insight into how some conditions often comorbid with schizophrenia arise. Further investigations examining the effect of potential mediating factors including smoking, alcohol consumption, and socioeconomic status may assist with future application of schizophrenia PRS in disease prediction or clinical practice.

Grant Support: US National Institute of Mental Health (MH116188), Chinese Scholarship Council (CSC201700260258).

\section{Magnitude of Gene-Environment Correlation for Cognitive Ability}

Anqing Zheng $^{1} \&$ Daniel A. Briley ${ }^{1}$

${ }^{1}$ Department of Psychology, University of Illinois at Urbana-Champaign, Champaign, IL, USA

Keywords Gene-environment correlation, Polygenic score, Extended twin model, Cognitive ability

Cognitive ability is a complex phenotype with numerous impacts on positive outcomes across the lifespan, from better health to higher income. Both quantitative genetic methods (e.g., the classical twin design) and molecular genetic methods (genome-wide association studies) indicate substantial heritability of cognitive ability. However, these methods typically assume the absence of gene-environment correlation $(r \mathrm{GE})$, despite essentially all theoretical models of educational attainment posing some role of $r \mathrm{GE}$ across development. Further, empirical evidence on genetic nurture demonstrated the importance of $r \mathrm{GE}$ for cognitive phenotypes. Yet, we do not have a general estimate of the magnitude of $r$ GE for cognitive ability. Here, we use data from the Adolescent Brain Cognitive Development study (ABCD; $\mathrm{N}=1681$ pairs) to estimate the correlation between additive genetic effects and the shared environment. These estimates are possible by integrating the classical twin design with a polygenic score for educational attainment (Dolan et al., 2021). We also include height as a negative control. Our study provides empirical evidence as to the magnitude of gene-environment interplay on a consequential life outcome and highlights a developmental cascade whereby people select or evoke environments that match their genetically influenced characteristics dynamically across early life.

Grant Support: Daniel A. Briley was supported by Jacobs Foundation Research Fellowships. 


\section{Heritable, Prenatal, and Postnatal Influences on Child Cortisol and Internalizing Symptom Development}

Anna M. Zhou ${ }^{1}$, Kristine Marceau ${ }^{2}$, Amanda M. Ramos ${ }^{3}$, Leslie D. Leve $^{4}$, Jody M. Ganiban ${ }^{5}$, Daniel S. Shaw ${ }^{6}$, David Reiss ${ }^{7}$, Misaki N. Natsuaki $^{8}$, Jenae M. Neiderhiser ${ }^{1}$

${ }^{1}$ Department of Psychology, The Pennsylvania State University, University Park, Pennsylvania, USA

${ }^{2}$ Department of Human Development and Family Studies, Purdue University, West Lafayette, Indiana, USA

${ }^{3}$ Department of Epidemiology, University of North Carolina-Chapel Hill, Chapel Hill, North Carolina, USA

${ }^{4}$ Prevention Science Institute, University of Oregon, Eugene, Oregon, USA

${ }^{5}$ Department of Psychological and Brain Sciences, George Washington University, Washington DC, USA

${ }^{6}$ Department of Psychology, University of Pittsburgh, Pittsburgh, Pennsylvania, USA

${ }^{7}$ Child Study Center, Yale University, New Haven, Connecticut, USA ${ }^{8}$ Department of Psychology, University of California, Riverside, Riverside, California, USA

\section{Keywords Internalizing symptoms, Prenatal distress, Cortisol}

Predictors of children's internalizing symptoms are often studied independently, including heritable risk, prenatal distress, postnatal parent internalizing symptoms and child hypothalamic-pituitaryadrenal (HPA) activity. Additionally, child HPA activity may mediate prenatal influences on child behavior, with more prenatal distress associated with lower cortisol. Using data from the Early Growth and Development Study ( $n=361)$, a prospective parent-offspring adoption design, we examined heritability, prenatal distress, and postnatal parental internalizing symptoms as predictors of children's morning cortisol levels and internalizing symptoms from childhood to early adolescence. Heritable risk was indexed by birth parent (BP) internalizing symptoms and diagnoses. Birth mothers reported on internalizing symptoms during pregnancy. Adoptive parents (AP) self-reported internalizing symptoms when children were 9-, 18-, and 27-months-old. At age 4.5, home collection of child cortisol occurred on three consecutive mornings within 30 minutes of waking. APs reported on child internalizing symptoms when children were age 4.5 , $6,7,8$, and 11. Using structural equation modeling, we found that BP internalizing and prenatal internalizing symptoms were not significantly associated with children's cortisol levels or internalizing symptoms. AP internalizing symptoms were associated with children's internalizing symptoms at age $4.5(\beta=0.90, p<.01)$. Linear trajectories indicated overall decreases in children's internalizing symptoms from ages 4.5 to 11 , with children's morning cortisol positively associated with children's internalizing symptom change (i.e., less decrease and flatter slope) $(\beta=0.16, p=.03)$. Results suggest that HPA axis activity is independently associated with change in internalizing symptoms. Postnatal parent symptoms are related to proximal assessments of children's internalizing symptoms, but not change over time.

Grant Support: R01 HD042608 NICHD; OBSSR, NIH; R01 M092118, NIMH; R56 HD042608, NICHD; K01 DA039288, NIDA; UH3 OD023389, Office of the Director, NIH
Using Genetic Information to Improve the Prediction of Food Choice: A Case Study of Alcoholic Beverages

Chen Zhu ${ }^{1,2}$, Timothy Beatty ${ }^{3}$, Qiran Zhao ${ }^{1,2}$, Wei $\mathrm{Si}^{1}$, and Qihui Chen ${ }^{1,2}$

${ }^{1}$ College of Economics and Management, China Agricultural University, Qinghuadonglu No. 17, Beijing, 100083, China

${ }^{2}$ China Center for Genoeconomic Studies (CCGS), Beijing, 100083, China

${ }^{3}$ Department of Agricultural and Resource Economics, UC Davis, CA 95616, USA

Keywords Consumer behavior, Food choice, Choice experiment, Genetic factor, Machine learning.

Individual food choices and consumption are closely relating to one's diet, nutrition, and health. Using the case of alcoholic beverages, this study extends the random-utility framework by incorporating genetic information into consumer demand models, and demonstrates the significant impact of genetic factors on individual food choice decisions in a novel way. Integrating individual-level responses of discrete choice experiments (DCE), genotyping data, and socioeconomic/demographic characteristics of 484 participants collected from face-to-face interviews in mainland China, we employ a machine learning-based classification (MLC) approach to identify and predict individual choices. We show that genetic factors are critical to explaining variations in both general drinking behavior and choices of particular products. The MLC predictive model with both socio-demographic and genetic features yields the highest accuracy of $74.7 \%$ and AUC-ROC of 0.85 . Our findings warrant further economic studies of human behaviors with the integration of genetic data.

Grant Support: This study was supported by the National Natural Science Foundation of China (No. 71973136, 71773122, and 71603261) and China Agricultural University.

\section{Shared Genetic and Phenotypic Risk Factors in Pain and psychological Conditions}

Katerina Zorina-Lichtenwalter ${ }^{1}$, Carmen Bango $^{2}$, Marta Čeko ${ }^{3}$, Naomi P. Friedman ${ }^{4}$, Matthew C. Keller ${ }^{5}$, Lukas Van Oudenhove ${ }^{6}$, Subrata $\mathrm{Paul}^{7}$, Tor D. Wager ${ }^{2}$

${ }^{1}$ Institute of Cognitive Science and Institute for Behavioral Genetics, University of Colorado Boulder, USA

${ }^{2}$ Department of Psychological and Brain Sciences, Dartmouth College, USA

${ }^{3}$ Department of Psychology and Neuroscience and Institute of Cognitive Science, University of Colorado Boulder, USA

${ }^{4}$ Department of Psychology and Neuroscience and Institute for Behavioral Genetics, University of Colorado Boulder, USA

${ }^{5}$ Department of Psychology and Neuroscience and Institute for Behavioral Genetics, University of Colorado Boulder, USA

${ }^{6}$ Department of Chronic Diseases, Metabolism, and Ageing, KU Leuven, Belgium

${ }^{7}$ Institute for Behavioral Genetics, University of Colorado Boulder, USA

Keywords Pain, Psychopathologies, Genetic correlations, SEM

Chronic pain and psychological conditions show substantial phenotypic and genetic overlap. Our goal was to find and characterize the shared genetic risk components underlying these conditions. Using the UK Biobank dataset, we selected chronic conditions marked by 
persistent pain across body sites and suspected etiologies. We ran a genome-wide association study (GWAS) on each condition and estimated their genetic correlations, which we compared to phenotypic correlations. Next, we performed confirmatory factor analysis (CFA) to test two hypotheses-anatomic and etiologic groupings of conditions-and exploratory factor analysis (EFA) followed by CFA. The EFA-CFA pipeline was validated using a split-genome approach with model discovery and validation in odd and even autosomes, respectively. All confirmatory analyses were performed using the genomic structural equation modeling (gSEM) framework. Furthermore, we plotted genetic correlations between all pain traits as a network, extracting graph properties. Our gSEM results show evidence of a bifactor structure, with a prominent general factor explaining most of the shared genetic variance and two specific factors with significant loadings from musculoskeletal and craniovisceral disorders. Network visualization reveals a large cluster of highly inter-connected conditions that share genetic associations. Including psychosocial traits into the phenotypic analysis showed an effect of neuroticism on correlations among symptom-based pain traits, which were likewise positively and negatively correlated with depression and happiness, respectively. Our findings suggest a common genetic predisposition for etiologically distinct pain conditions, which may indicate shared pathophysiology. Overall, we report evidence for chronic pain as a systemic condition modulated by psychosocial traits.

Grant Support: NIH PainGen R01 (DA046064).

Publisher's Note Springer Nature remains neutral with regard to jurisdictional claims in published maps and institutional affiliations. 